\title{
Psychopathic traits in Dutch adolescent offender and community samples : relationships with gender, age, institutional misbehavior, substance use and interpersonal style.
}

Citation for published version (APA):

Das, J. (2008). Psychopathic traits in Dutch adolescent offender and community samples : relationships with gender, age, institutional misbehavior, substance use and interpersonal style. [Doctoral Thesis, Maastricht University]. Universiteit Maastricht. https://doi.org/10.26481/dis.20081121jd

Document status and date:

Published: 01/01/2008

DOI:

10.26481/dis.20081121jd

Document Version:

Publisher's PDF, also known as Version of record

Please check the document version of this publication:

- A submitted manuscript is the version of the article upon submission and before peer-review. There can be important differences between the submitted version and the official published version of record. People interested in the research are advised to contact the author for the final version of the publication, or visit the DOI to the publisher's website.

- The final author version and the galley proof are versions of the publication after peer review.

- The final published version features the final layout of the paper including the volume, issue and page numbers.

Link to publication

\footnotetext{
General rights rights.

- You may freely distribute the URL identifying the publication in the public portal. please follow below link for the End User Agreement:

www.umlib.nl/taverne-license

Take down policy

If you believe that this document breaches copyright please contact us at:

repository@maastrichtuniversity.nl

providing details and we will investigate your claim.
}

Copyright and moral rights for the publications made accessible in the public portal are retained by the authors and/or other copyright owners and it is a condition of accessing publications that users recognise and abide by the legal requirements associated with these

- Users may download and print one copy of any publication from the public portal for the purpose of private study or research.

- You may not further distribute the material or use it for any profit-making activity or commercial gain

If the publication is distributed under the terms of Article 25fa of the Dutch Copyright Act, indicated by the "Taverne" license above,

Download date: 26 Apr. 2023 


\title{
PSYCHOPATHIC TRAITS Relationships WITH GENDER, AGE, IN DUTCH ADOLESCENT institutional OFFENDER AND substance use and COMMUNiTy SAMPLES style
}

\author{
JACKY DAS
}


This research was financed by the Dutch Ministery of Justice. The printing of this thesis was financed by Maastricht University and De Waag.

Lay-out: Wietske Lute

Printed by: Ponsen \& Looijen

๑ 2008, Jacky Das, The Netherlands

All rights reserved. Save exceptions stated by the law, no part of this publication may be reproduced, stored in a retrieval system of any nature, or transmitted in any form or by any means, electronic, mechanical, photocopying, recording or otherwise, included a complete or partial transcription, without the prior written permission of the authors, application for which should be addressed to author. 


\title{
Psychopathic traits in Dutch adolescent offender and community samples:
}

Relationships with gender, age, institutional misbehavior, substance use and interpersonal style

\author{
Proefschrift
}

ter verkrijging van de graad van doctor

aan de Universiteit van Maastricht, op gezag van de Rector Magnificus,

Prof. mr. G.P.M.F. Mols

Volgens het besluit van het College der Decanen, in het openbaar te verdedigen

op vrijdag 21 november 2008 om 12.00 uur

door

Jacqueline Das 
Promotores: prof. dr. C. de Ruiter

prof. dr. Th. A.H. Doreleijers, Vrije Universiteit 
Beoordelingscommissie: prof. dr. A. Arntz, voorzitter

dr. D. Bernstein

prof. dr. A. Korebrits

prof. dr. Ch. Van Nieuwenhuizen,

Universiteit van Tilburg

prof. dr. H.L.I. Nijman,

Radboud Universiteit Nijmegen

prof. dr. R. Vermeiren, Univeristeit Leiden 
Change will not come if we wait for some other person or some other time. We are the ones we've been waiting for. We are the change that we seek.

Barack Obama 
P.11 CHAPTER 1

General Introduction

P. 41 CHAPTER 2

Reliability and Construct

Validity of the Dutch

Psychopathy Checklist: Youth

Version: Findings From a

Sample of Male Adolescents in

a Juvenile Justice Treatment

Institution

P.69 CHAPTER 3

Reliability and Validity of

the Psychopathy Checklist:

Youth Version in Dutch Female

Adolescents

P.93 CHAPTER 4

The Dutch Psychopathy

Checklist: Youth Version:

Structural and Metric Validity
P. 119 CHAPTER 5

Predictive Validity of the

Dutch PCL:YV for Institutional

Disruptive Behavior: Findings

From Two Samples of Male

Adolescents in a Juvenile Justice

Treatment Institution

P. 143 CHAPTER 6

The Youth Psychopathic Traits

Inventory: Psychometric

Properties and Its Relation to

Substance Use and Interpersonal

Style in a Dutch Sample of Non-

referred Adolescents

P. 163 CHAPTER 7

General Discussion 
Voor Justin, Jort en Noure 
This thesis is based on the following papers:

Das, J., Hillege, S., Ruiter, C. de, Hildebrand, M., Chakhssi, F., \& Doreleijers, Th. (under review). The Dutch Psychopathy Checklist: Youth Version: Structural and metric validity. Behavioral Sciences and the Law.

Das, J., Ruiter, C. de, \& Doreleijers, Th. (2008). Reliability and validity of the Psychopathy Checklist: Youth Version in Dutch female adolescents. International Journal of Law and Psychiatry, 31, 219-228.

Das, J., Ruiter, C. de, Doreleijers, Th., \& Hillege, S. (accepted). Reliability and validity of the Dutch Psychopathy Checklist: Youth Version in a sample of male adolescent offenders. Assessment.

Das, J., Ruiter, C. de, Lodewijks, H., \& Doreleijers, H. (2007). Predictive validity of the Dutch PCL:YV for institutional disruptive behavior: Findings from two samples of male adolescents in a juvenile justice treatment institution. Behavioral Sciences and the Law, 2, 739-755.

Hillege, S., Das, J., \& Ruiter, C. de (under review). The Youth Psychopathic Traits Inventory: Psychometric properties and its relation to substance use and interpersonal style in a Dutch sample of nonreferred adolescents. Journal of Adolescence. 

Professionals at work in Dutch forensic psychiatric institutions are becoming increasingly aware of the need for research into the characteristics of the mentally disordered offenders in their care, and the identification of factors related to risk of future violence (i.e., criminogenic factors for violence). According to the so-called What Works framework, risk, need, and responsivity are crucial principles to be considered in every forensic psychiatric treatment (Andrews, 1995; Andrews \& Bonta, 2003). Treatment is most effective when delivered proportionally to the level of risk (risk principle). The risk principle is defined as the probability of criminal reoffending, which is determined by the number and severity of risk factors. The need principle suggests that, in order to be effective, treatment should be directed at the offender's changeable criminogenic needs, such as substance abuse, aggressive interpersonal style and impulsivity. Finally, the responsivity principle implies that particular characteristics of the individual (for instance, personality and cognitive styles) should be taken into account when tailoring treatment to the individual offender. Andrews, Bonta and Hoge (1990) conducted a meta-analysis in order to examine the relationship of the risk-need-responsivity principles to treatment outcome. They found that treatment programs which complied with the risk-needresponsivity principles had the largest effect sizes; that is, they were most effective in reducing recidivism. Criminal sanctioning without the delivery of treatment services or making use of inappropriate treatment programs, had no effect or a small negative effect on recidivism (Andrews et al., 1990).

\section{Psychopathy in the What Works framework}

Psychopathy is a socially devastating disorder defined by a constellation of interpersonal, affective, and behavioral characteristics (Wong \& Hare, 2005). These include egocentricity; impulsivity; irresponsibility; shallow emotions; lack of empathy, guilt, or remorse; pathological lying; manipulativeness; and the persistent violation of social norms and expectations (Cleckley, 1976; Hare, 1998). The clinical construct of psychopathy is relevant for the riskneed-responsivity principles in several ways. First, because of its significant 
and robust association with institutional infractions (Edens, Petrila, \& Buffington-Vollum, 2001), violent behavior (Fort \& Burke, 1998; Hare, McPherson, \& Forth, 1988), and general criminal recidivism (Salekin, Rogers, \& Sewell, 1996; Hemphill, Hare, \& Wong, 1998; Hildebrand, de Ruiter, \& de Vogel, 2004), the concept of psychopathy should always be considered when determining the level of risk according to the risk principle. In line with these findings, psychopathy is included as a risk factor in most structured risk assessment instruments, including the Historical, Clinical, Risk Management-20 (HCR-20; Webster, Douglas, Eaves, \& Hart, 1997) for general violence and the Sexual Violence Risk-20 (SVR-20; Boer, Hart, Kropp, \& Webster, 1997) for sexual violence.

Second, the need principle implies that the targets of treatment interventions should be based on a thorough evaluation of changeable criminogenic needs. According to Wong and Hare (2005) eight criminogenic needs are linked to violence in the psychopath, including: 1) social and peer support of antisocial behavior; 2) dysfunctional attitudes and behaviors and their association with violence; 3) dysfunctional emotions and the lack of emotional control; 4) failure to accept responsibility for own actions; 5) substance abuse; 6) lack of work ethic, employment skills and appropriate leisure activities; 7) antisocial peers, networks, and subculture in the community; and 8) being abused as a child (optional). In order to reduce the risk of future violence, these criminogenic needs should be targeted by treatment.

Third, it has been demonstrated that psychopaths show a profound lack of motivation for treatment (Ogloff, Wong, \& Greenwood, 1990), and poor treatment compliance (Hildebrand, De Ruiter, \& Van Zaane, submitted). Furthermore, higher psychopathy scores are associated with lower scores on global measures of change (Hobson, Shine, \& Roberts, 2000; Ogloff et al., 1990), and some types of treatments may have iatrogenic effects (Hobson et al., 2000; Rice, Harris, \& Cormier, 1992). The responsivity principle states that, in order to be effective, interventions must be congruent with the offender's capacities and learning style. An impressive body of evidence has 
identified psychopaths as having deficiencies in affective responsiveness (Blair, 2004) and in the ability to make use of contextual information to modulate goal-directed behavior (Newman, 1998; Newman, Schmitt, \& Voss, 1997). Treatment delivery should include interventions which accommodate these deficiencies. In addition, clinicians should consider the interpersonal characteristics of psychopathy, including superficial charm, grandiosity, lying and manipulation, in the establishment of a functional therapeutic alliance (Wong \& Hare, 2005). The relatively high prevalence of psychopathy in forensic psychiatric samples (15-30\%; Hare, 1991; Hart, Hare, \& Forth, 1994: Hildebrand \& De Ruiter, 2004) implies that the assessment of psychopathic traits should be a standard part of forensic assessment and treatment.

\section{Psychopathy and the treatment of Dutch adolescent offenders}

In The Netherlands, recidivism rates of various offender groups are monitored on a yearly basis by the WODC (scientific research and documentation center of the Ministry of Justice). Based on results from this monitor, Wartna, Harbachi, and Van der Laan (2005) demonstrated that four years after release from a juvenile justice treatment institution, $62 \%$ of the adolescents had recidivated with a serious offense (reconviction for a crime punishable by four years or more). Although this study does not provide information on the effects of the different types of treatment that were provided, it is warranted to conclude that, overall, juvenile justice treatment institutions seem not very effective in reducing recidivism among juvenile offenders. Moreover, the Inspection for Child Welfare (Inspectie Jeugdzorg; 2007) concluded that the assessment and treatment in juvenile justice treatment institutions was inadequate and there was a serious lack of physical safety for youngsters as well as for group leaders. In addition, the National Audit Office (Algemene Rekenkamer; 2007) presented disturbing findings regarding the efficiency of juvenile justice institutions in reducing criminal recidivism. In the first place, the Audit Office concluded that juvenile justice institutions do not provide treatment that is designed to systematically address the adolescents' criminogenic needs. In the second 
place, institutions do not systematically record improvements made in treatment nor the change in risk of future criminal behavior. In the third place, and in contrast to what has been statutorily stipulated, aftercare was provided only for a small subset of adolescents. Finally, most of the interventions offered in these institutions are not supported by empirical evidence and have not been officially recognized by the Dutch Commission for Behavior Interventions (Erkenningscommissie Gedragsinterventies Justitie; http://www.justitie.nl/).

The risk-need-responsivity principle provides an important framework for the improvement of treatment for juvenile offenders. For instance, Andrews and Dowden (2006) demonstrated in a meta-analysis that the effect of the risk principle on the effectiveness of treatment is even stronger for adolescents than for adults. This means that especially in adolescents it is important to provide intensive treatment for high-risk cases, whereas lowrisk cases have a low probability of negative outcome even in the absence of service. Furthermore, like in adults, the concept of psychopathy may also prove to have a surplus value in the assessment of risk, the identification of criminogenic needs, and the appropriate delivery of treatment interventions to juvenile offenders.

\section{Psychopathy in children and adolescents}

The Diagnostic and Statistical Manual of Mental Disorders-fourth edition (DSM-IV; American Psychiatric Association, 1994) requires a person to be at least 18 before a diagnosis of a personality disorder can be made. However, like all personality disorder traits, characteristics of psychopathy do not suddenly emerge in early adulthood. Psychopathic traits have been demonstrated to become evident early in life and develop throughout childhood and adolescence. For example, a psychopath's propensity for instrumental aggression and violence has been shown to begin early in life and continues throughout the lifespan (Forth \& Burke, 1998; Forth, Kosson, \& Hare, 2003). In fact, the Psychopathy Checklist-Revised (PCL-R; Hare, 1991, 2003), the gold standard for the assessment of psychopathy in adults, 
includes several items that relate to behavior before the age of 18 (i.e., item 12: Early behavior problems; item 18: Juvenile delinquency). Several scholars have argued that the identification of psychopathic traits in childhood or adolescence may offer a better understanding of the etiology of the disorder and may provide starting-points for targeting interventions (Forth, Hart, \& Hare, 1990; Forth \& Mailloux, 2000; Frick, Bodin, \& Barry, 2000).

\section{Instruments}

Various instruments have been developed for the assessment of psychopathic traits in youth (for a detailed summary see: Kotler \& McMahon, 2005). For the assessment of psychopathic traits in adolescents aged 12 to 18 years, mainly the Psychopathy Checklist: Youth Version (PCL:YV; Forth et al., 2003) is used. The PCL:YV was adapted from the PCL-R (Hare, 1991, 2003). The PCL: YV is a 20-item checklist (see Table 1), which is completed on the basis of a semi-structured interview and detailed collateral file information. A Dutch version of the PCL:YV (Psychopathie Checklist: Jeugd Versie; PCL: JV; De Ruiter, Kuin, de Vries, \& Das, 2002) is available for research purposes, and will be published by Harcourt Test Publishers.

The PCL:YV is a diagnostic tool, designed to provide a comprehensive view of an adolescent's psychopathic traits. The PCL:YV is a time-consuming instrument and because of its methodology (i.e., scoring by trained mental health professionals based upon interview and collateral information), it is of limited use in non-offender populations (because collateral documentation is often unavailable). As a result, several screening measures have been developed, including the Youth Psychopathic traits Inventory (YPI; Andershed, Kerr, Stattin, \& Levander, 2002; Dutch authorized translation: Das \& De Ruiter, 2003), the Childhood Psychopathy Scale (CPS; Lynam, 1997), and the Antisocial Process Screening Device (APSD; Frick and Hare, 2001; Dutch version: De Ruiter, Van Domburgh, \& Das, 2003). These other psychopathy measures for children and adolescents will be discussed briefly here (see Table 2). 
TABLE 1

ITEMS OF THE HARE PSYCHOPATHY CHECKLIST: YOUTH VERSION

1. Impression management

2. Grandiose sense of self worth

3. Stimulation seeking

4. Pathological lying

5. Manipulation for personal gain

6. Lack of remorse

7. Shallow affect

8. Callous / lack of empathy

9. Parasitic orientation

10. Poor anger control

11. Impersonal sexual behavior

12. Early behavior problems

13. Lacks goals

14. Impulsivity

15. Irresponsibility

16. Failure to accept responsibility

17. Unstable interpersonal relationships

18. Serious criminal behavior

19. Serious violations of conditional release

20. Criminal versatility

The YPI is a 50-item self-report measure of psychopathic traits in adolescents. One of the strengths of the YPI is that each psychopathic trait is measured with several items (Andershed et al., 2002). Furthermore, the items are worded in such a way that the traits sound positive to a person with psychopathic traits but not to those without them (see Chapter 6 for some example items). The CPS was also designed to measure psychopathic 
traits in adolescents (Lynam, 1997; Falkenbach, Poythress, \& Heide, 2003). It can be used as a self-report measure but it can also be rated by teachers and/or parents. The CPS is theoretically based on the PCL-R but the items are derived from the Child Behavior Checklist (CBCL; Achenbach, 1991) and the Common-Language Q-sort (CCQ; Caspi et al., 1992). Finally, for the measurement of psychopathic traits in children younger than age 12 , Frick and Hare (2001) developed the Antisocial Process Screening Device (APSD; Dutch version: De Ruiter et al., 2003). The APSD includes 20 items, which are rated by the parent and the teacher of the child. Several studies have indicated that the APSD can also be successfully used as a self-report instrument for measuring psychopathic traits in adolescents (Caputo, Frick, \& Brodsky, 1999; Frick et al., 2000; Poythress, Dembo, Wareham, \& Greenbaum, 2006; Silverthorn, Frick, \& Reynolds, 2001).

TABLE 2

CHILD AND ADOLESCENT PSYCHOPATHY MEASURES, INCLUDING EXAMPLE ITEMS

\begin{tabular}{lllll}
\hline Measure & Coder & Age range & Number & Example item \\
& & (years) & of items & \\
\hline PCL:YV & Trained professional & $12-18$ & 20 & 1. Impression management \\
APSD & Parent, teacher, adolescent & $6-18$ & 20 & 8. Brags excessively \\
YPI & Adolescent & $12-18$ & 50 & 1. Its easy for me to charm \\
& & & & and seduce others to get \\
CPS & Parent, teacher, adolescent & $12-18$ & 13 & what I want from them \\
& & & & 3. Acts charming to get \\
& & & & his way
\end{tabular}

The following case vignette illustrates the way psychopathic traits are expressed in 'real world' behavior. 


\section{Intermezzo: Case Vignette}

Harry is an 18-year old boy of Dutch origin. He was raised in a family with two children: Harry and his younger brother. After his parents divorced when he was six years old, Harry lived with his mother; contact with his father was limited.

Starting from a young age, Harry was an overactive and impulsive child, who could not be easily corrected (item 12 of the PCL:YV). In the beginning of adolescence, his problem behavior increased and Harry became unmanageable at home as well as at school. He started living according to his own rules, often lied (item 4 ), and demonstrated intimidating (item 1), aggressive (item 10) and manipulative (item 4) behavior. He also began using large amounts of drugs and alcohol (item 3). He didn't seem to care about the consequences of his actions (item 8). Harry was placed in a residential welfare institution. In the institution, Harry continued his problematic behavior and was hard to manage. $\mathrm{He}$ was calculating in his behavior and appeared only interested in fulfilling his own wants and needs (item 5). After Harry threatened his therapist with a knife (items $18 \& 20$ ), the court convicted him to a mandatory treatment order and Harry was placed in a secure juvenile justice treatment institution. At the time, he was sixteen years old. Harry resided in the institution for a year and a half. Although the treatment staff reported some progress in his behavior, success was limited and of short duration (item 16). Newly learned behavior did not seem to be maintained. Harry was capable of identifying his problems and he seemed to know what alternative behaviors would be appropriate. However, he did not use this knowledge to actually modify his behavior (item 16). Although the core pathology was still present after a year and a half, the treatment staff concluded that Harry would not benefit from further treatment in a secure institution. It was decided to transfer Harry to an open treatment setting, which would allow him opportunities to learn to deal with a variety of situations in daily life, while still being under the supervision of the treatment staff. 


\section{Construct validity of psychopathy: YPI, APSD and CPS}

The concept of the nomological network (Cronbach \& Meehl, 1955) can be used as a theoretical framework for understanding the construct validity of adolescent psychopathy. In this framework, constructs are continually redefined and clarified through their relationships with other constructs. The first layer of the nomological network surrounding psychopathy involves the interrelationship between various measures of psychopathy (Benning, Patrick, Salekin, \& Leistico, 2005). The construct validity is supported when these measures strongly relate to each other. In several studies, limited convergence has been demonstrated between the PCL:YV and other measures of psychopathic traits in adolescence (Lee, Vincent, Hart, \& Corrado, 2003; Murrie \& Cornell, 2002; Salekin, Leistico, Trobst, Schrum, \& Lochman, 2005; Skeem \& Cauffman, 2003). A method effect (self report versus clinician ratings) was demonstrated to underlie this lack of convergence (Lee et al., 2003), suggesting that these measures do not capture the concept in the same manner. In contrast to these findings, Andershed, Hodgins and Tengström (2007) recently found that correlations between total scores and conceptually corresponding factor scores of the PCL:YV and the YPI were moderate. Furthermore, in this study support was provided for the convergent validity of the categorical diagnosis of psychopathy.

The second layer in the nomological network is the relationship of psychopathy measures with measures of other personality disorders (Benning, Patrick, Salekin, et al., 2005). Construct validity is supported when psychopathy measures are strongly associated with related disorders, while demonstrating divergent relationships with unrelated disorders. Research with the PCL:YV as well as the YPI and APSD has demonstrated that boys with psychopathic traits show an earlier onset of antisocial behavior as well as more, a greater variety of and more severe Conduct Disorder (CD) symptoms than boys without these traits (Andershed et al., 2002; Christian, Frick, Hill, Tyler, and Frazer, 1997; Forth et al., 1990; Kosson, Cyterski, Steuerwald, Neumann, \& Walker-Matthews, 2002; Myers, Burket, \& Harris, 1995; Rogers, Johansen, Chang, \& Salekin, 1997). Furthermore, male 
adolescent first-time offenders who scored high on the CPS were found to be more impulsive, and more prone to externalizing behaviors, whereas they were less prone to internalizing behaviors than boys with a low CPS score (Lynam, 1997).

Finally, the third layer of the nomological network involves the relationship of psychopathy measures with normal-range personality dimensions (Benning, Patrick, Salekin, et al, 2005). Construct validity is supported when psychopathy measures demonstrate theoretically meaningful relationships with relevant personality dimensions. Several researchers have demonstrated that the construct of adolescent psychopathy could be meaningfully placed within a personality framework as demonstrated by associations of the PCL:YV with several personality instruments. For instance, psychopathic traits were found to be related to amoral rebelliousness and impulsive tendencies as measured by the Minnesota Multiphasic Personality Inventory-Adolescent version (MMPI-A) (Brandt, Kennedy, Patrick, \& Curtin, 1997) and to the Big Five factors Agreeableness and Conscientiousness (Salekin et al., 2005). Furthermore, psychopathy scores were positively related to an emotionally cold (Salekin et al., 2005) and dominant (Forth, Brown, Hart, \& Hare, 1996) interpersonal style. However, some findings have been equivocal. For instance, while a lack of anxiety and negative affectivity is supposed to be a central feature of psychopathy (for a review, see Skeem, Mulvey, \& Grisso, 2003), PCL:YV scores were either not related to (Brandt et al., 1997; Skeem \& Cauffman, 2003) or even positively related to negative affectivity (Kosson et al., 2002). It should be noted here that the YPI has been found to show a theoretically coherent inverse relationship with anxiety in institutionalized young offenders, (Skeem \& Cauffman, 2003).

\section{Dimensions of psychopathy}

Initially, adult psychopathy (Harpur, Hakstian, \& Hare, 1988), as well as adolescent psychopathy (e.g., Brandt et al., 1997; Forth \& Mailloux, 2000) were considered to be underpinned by two distinct but correlated factors. The first factor is characterized by a selfish, callous interpersonal style and a 
lack of remorse and empathy, and the second factor by a chronically unstable and antisocial lifestyle (Hare et al., 1990). In adults, the original two factors (Harpur et al., 1988) have been demonstrated to show differential correlations with external criteria, including psychiatric diagnoses, personality scale scores, emotional processing performance, and recidivism and violence (Harpur, Hare, \& Hakstian, 1989; Hemphill et al., 1998; Hildebrand \& De Ruiter, 2004). Recent research in adults (Cooke \& Michie, 2001; Hare, 2003) and adolescents (Kosson et al., 2002; Neumann, Kosson, Forth, \& Hare, 2006; Vincent, 2002), however, suggests that the original Factor 1 should be broken down into two factors representing an arrogant and deceitful interpersonal style (new Factor 1), and a deficient affective experience (new Factor 2). A new Factor 3 comprises impulsive behavioral traits and an irresponsible lifestyle. Considerable debate exists about whether criminal behaviors should be included in the construct of psychopathy. For instance, Cooke, Michie, Hart, and Clark (2004) have argued that the interpersonal, affective, and impulsive lifestyle dimensions are core symptoms, whereas the antisocial/criminal symptoms should be perceived as consequences of the core psychopathy traits. In contrast, others have found that antisocial tendencies play a fundamental role in the psychopathy concept (Hare, 2003; Neumann, Vitacco, Hare, \& Wupperman, 2005).

In adults, the four-factor model was found to be superior to the threefactor model in predicting violence (Hill, Neumann, \& Rogers, 2004; Vitacco, Neumann, \& Jackson., 2005). Furthermore, all four psychopathy dimensions (i.e., interpersonal, affective, lifestyle and antisocial) were found to be differentially associated with personality, behavior, and adaptive functioning (Hall, Benning, \& Patrick, 2004). Of the youth psychopathy measures, only the PCL:YV includes antisocial items, whereas the other youth measures do not (YPI, CPS) or include a limited number of antisocial items (APSD; one item). Using the CPS, Salekin et al. (2005) found that the interpersonal, affective and lifestyle dimensions showed differential relations with Big Five personality dimensions and interpersonal styles. However, a comprehensive test of the construct validity of all four factors of the PCL:YV has not yet been performed.

Ultimate decisions about the importance of the antisocial items for the 
measurement of psychopathic traits in youth cannot be made until research has extensively examined the contribution of this dimension to the overall nomological network surrounding psychopathy. In the meantime, use of the Hare's (2003) four-factor model (see Table 3) in research is attractive in terms of overall comprehensiveness, since it incorporates the three Cooke and Michie factors, and at the same time includes a robust antisocial factor (Forth et al., 2003).

TABLE 3 .

HARE'S (2003) FOUR-FACTOR MODEL DERIVED FROM THE PCL-R

Factor 1: Interpersonal

1. Glibness/superficial charm

2. Grandiose sense of self-worth

4. Pathological lying

5. Conning/manipulative

Factor 2: Affective

6. Lack of remorse or guilt

7. Shallow affect

8. Callous/lack of empathy

16. Failure to accept responsibility for own actions

Factor 3: Lifestyle

3. Need for stimulation/proneness to boredom

9. Parasitic lifestyle

13. Lack of realistic, long-term goals

14. Impulsivity

15. Irresponsibility

Factor 4: Antisocial

10. Poor behavioral controls

12. Early behavioral problems

18. Juvenile delinquency

19. Revocation of conditional release

20. Criminal versatility 


\section{Psychopathy and antisocial behavior}

Parallel to research in adult samples (e.g., Hart, 1998; Hemphill et al., 1998), psychopathy scores are consistently found to be related to a variety of indices of criminal offending in adolescents. Boys with psychopathic traits demonstrate more charges for severe violent and non-violent offenses in the past, more participation in different types of illegal activity in the past, and they are more likely to use weapons (Christian et al., 1997; Forth et al., 1990; Kosson et al., 2002; Murrie, Cornell, Kaplan, McConville, \& Levy-Elkon, 2004; Pohthress et al., 2006; Salekin, Neumann, Leistico, DiCicco, \& Duros, 2004) than boys without these traits. Furthermore, male adolescents with psychopathic traits demonstrate more (violent) disruptive behavior while institutionalized (Hicks, Rogers, \& Cashel, 2000; Rogers et al., 1997).

Psychopathy scores also predict the likelihood of future offenses (Falkenbach et al., 2003; Forth et al., 1990; Gretton, Hare, \& Catchpole, 2004; Gretton, McBride, O'Shaughnessy, \& Kumka, 2001). Furthermore, Gretton et al. (2003) demonstrated that male adolescent offenders with high PCL: YV scores (29 or greater) violently reoffended significantly sooner than those with low (less than 18) or medium (18-28) PCL:YV-scores. With respect to type of offenses, some studies demonstrated psychopathic traits to be more strongly related to violent recidivism than to nonviolent recidivism (Forth et al., 1990; Långström \& Grann, 2002; Gretton et al., 2004), while other studies have demonstrated the opposite (Brandt et al., 1997; Gretton et al., 2001). Recently, Edens, Campbell and Weir (2007) performed a meta-analysis of research with the PCL:YV in 21 adolescent samples (total $\mathrm{N}=2,867$ ) and demonstrated weighted mean effect sizes $\left(r_{w}\right)$ of .24 and .25 for general and violent recidivism, respectively. A remarkable finding was that psychopathy scores were found to be equally predictive of general and violent recidivism as a more extensive risk assessment instrument, the Youth Level of Service / Case Management Inventory (YLS/CMI), suggesting “a lone personality construct can perform similarly to a more comprehensive risk assessment instrument" (p. 23, Edens et al., 2007). In contrast to what has been found in adults (Hildebrand et al., 2004; Rice \& Harris, 1997), the level of psychopathic 
traits was found to be only weakly related to sexual offense recidivism (Gretton et al., 2001). It should be noted, however, that the low base rate of sexual reoffending (15\%) in the Gretton et al. study may partially account for this weak relation.

\section{Psychopathy in female adolescents}

In the PCL:YV manual it is suggested that the PCL:YV can be applied invariantly across gender (Forth et al., 2003). However, this suggestion may not be warranted considering recent research findings in female adult samples (e.g., Salekin, Rogers, \& Sewell, 1997; Vitale \& Newman, 2001b; Vitale, Smith, Brinkley, \& Newman, 2002). The most striking finding is a lower prevalence rate of psychopathy in female adult forensic samples (Forth et al., 1996; Grann, 2000; Salekin et al., 1997; Salekin, Rogers, Ustad, \& Sewell, 1998; Vitale et al., 2002), than what is typically reported in male adult forensic samples (Hare, 1991; Hart et al., 1994). Parallel gender differences in prevalence rates have been found in adolescents by Schrum and Salekin (2006), who demonstrated a lower prevalence rate of psychopathic traits in detained girls than what has been found for male adolescents. Furthermore, their results indicated that some PCL:YV items may be less applicable to girls, including Poor anger control, Serious violations of conditional release, and Parasitic lifestyle. In contrast, some items that have not been found to be good disciminators in adult samples (Hare, 2003) were highly discriminant of psychopathy in girls, including Impersonal sexual behavior, Juvenile delinquency, and Unstable interpersonal relationships (Salekin $\&$ Schrum, 2006). Regarding the predictive value of psychopathic traits, Marsee, Silverthorn and Frick (2005) demonstrated that there were no gender differences for the interpersonal, affective, and lifestyle dimensions in their associations with aggression and delinquency in a non-referred sample of boys and girls ranging in age from 10 to 17 . However, the total psychopathy score, as well as each psychopathy dimension, showed a significantly stronger relation to interpersonal aggression in girls than in boys. Contrasting results were found by Odgers, Repucci, \& Moretti (2005) in their study of 125 girls 
incarcerated at a correctional facility. Using a structural equation modeling approach, only a moderate relationship between psychopathy scores and concurrent aggression could be identified. Moreover, it was demonstrated that PCL:YV scores were not predictive of future re-offending in the community in girls, whereas prior victimization experiences were.

\section{The etiology of psychopathy}

An extensive body of research has documented a two-pathway model for the development of conduct problems: a childhood-onset pathway and an adolescent-onset pathway (Lahey et al., 1998; Moffit, 1993). The childhoodonset pathway is characterized by the onset of conduct problems in the preschool early school-age years, and by a high degree of continuity throughout childhood and into adolescence and adulthood. Substantive evidence exists that psychopathic traits (Callous/Unemotional traits as measured by the APSD) are primarily prevalent within the childhoodonset subgroup and not the adolescent-onset subgroup (Frick \& Ellis, 1999; Lynam, 1996; Silverthorn \& Frick, 1999). This has led authors to suggest that psychopathic traits may help to identify a more homogeneous subgroup of children within the childhood-onset group with a distinctive etiology of conduct problems (Frick, 1998). In line with this assertion, research has demonstrated that Conduct Disordered children with CU traits are characterized by reduced physiological reactivity to visual threat cues (Blair, 1999), by deficits in moral reasoning (Blair, Monson, \& Frederickson, 2001), by lack of response facilitation for recognition of negative words (Loney, Frick, Clements, Ellis, \& Kerlin, 2003) and reward dominance in a passive-avoidance learning task (O’Brien \& Frick, 1996).

Blair (2005) has extensively reviewed the hypotheses that have been formulated on the processes which may underlie the development of psychopathic traits. A common element in these hypotheses is that they all focus on emotional detachment as the core symptom of psychopathy. These include a) the frontal lobe dysfunction hypothesis, b) the response modulation hypothesis, and c) the fear dysfunction hypothesis. The first 
hypothesis suggests that since frontal lobe dysfunction is related to antisocial behavior, it may lie at the basis of psychopathy (Raine, 2002). The second hypothesis states that psychopaths show impairments in response modulation (Lorenz \& Newman, 2002). More specifically, they show reduced automatic processing of information that is peripheral to their dominant response set. Because this information enables an individual to monitor and regulate behavior, psychopaths show impairments in self-regulation. Finally, the fear dysfunction hypothesis postulates that psychopathy is characterized by an impairment in neurobiological systems modulating fear behavior. Indeed psychopaths have been demonstrated to show impairments in a wide range of functions mediated by fear systems, including aversive conditioning (Flor, Birbaumer, Hermann, Ziegler, \& Patrick, 2002), generating autonomic responses to anticipated threat stimuli (Ogloff \& Wong, 1990), the augmentation of the startle response to anticipated threat (Levenston, Patrick, Bradley, \& Lang, 2000), passive avoidance learning (Newman \& Kosson, 1986), response reversal/extinction (Mitchel, Colledge, Leonard, \& Blair, 2002), and reactivity to visual threat cues (Blair, 1999). A variant of the fear dysfunction model was proposed by Blair (1995). He proposed a Violence Inhibition Mechanism (VIM) in humans that is activated by environmental distress cues in other people (e.g., crying in young children). The activation of the VIM, generates an aversive emotional reaction leading to withdrawal or a behavioral inhibition response. This mechanism is thought to be dysfunctional in psychopaths, thereby impairing moral socialization. In other words, the child with callous-unemotional traits does not experience an aversive emotional response to punishment.

Recently, Blair, Peschardt, Budhani, Mitchell \& Pine (2006) have proposed an explanatory model for the development of psychopathy, providing an integration of findings derived from the three hypotheses discussed above. In this model, psychopathy is regarded as a neurodevelopmental disorder and a cognitive neuroscience perspective is used to explain psychopathy at the ultimate causal (genetic or social ), molecular, neural, cognitive, and behavioral level. 
At the ultimate causal level, it is suggested that a genetic contribution is a prerequisite for the development of psychopathy. In fact, recent research on twins has indicated significant heritability and little shared environmental influence on what were called the 'fearless dominance' (Blonigen, Carlson, Krueger, Patrick, 2003; Blonigen, Hicks, Krueger, Patrick, Iacono, 2005), and 'callous and unemotional' aspects of psychopathy (Viding, Blair, Moffit, \& Plomin, 2005). In contrast, shared environment was found to be of significant influence on the behavioral aspects of psychopathy. In addition, Blonigen, Hicks, Krueger, Patrick and Iacono (2006) provided further evidence of distinct developmental pathways of the personality and behavioral dimensions of psychopathy in a longitudinal-epidemiological sample of male and female twins. They demonstrated that the fearless dominance aspect of psychopathy was stable over time, whereas the impulsivity-antisociality aspect was not. In addition, findings demonstrated greater genetic contributions to stability and greater nonshared environmental contributions to change over time.

It seems plausible that the genetic basis of psychopathy gives rise to effects at the molecular level. Although research is scarce and inconclusive, it is suggested that high levels of antisocial behavior may be associated with reduced norepinephrine levels (Raine, 1993). In addition, a reduced serotonergic response has been found to be related to impulsive and antisocial conduct, but not to the emotional basis of psychopathy (Dolan \& Anderson, 2003).

Research at the neural system level clearly suggests that the amygdala play a role in the impairments in emotional information processing that are characteristic of psychopaths. More specifically, dysfunction of the amygdala may disrupt aversive conditioning, the augmentation of the startle reflex by visual threat primes, passive avoidance learning, and recognition of fearful expressions. Frontal lobe dysfunction has also been related to psychopathy in particular, and to antisocial behavior in general. Neurological research into frontal lobe dysfunction, indicates that only lesions of the orbital and ventrolateral cortex, and not the prefrontal dorsolateral cortex are associated 
with increased risk of aggression (Grafman, Schwab, Warden, Pridgen, \& Brown, 1996). Furthermore, the orbito-ventrolateral frontal cortex has been found to increase the risk of reactive aggression, but does not account for the instrumental aggression often seen in psychopaths.

At the cognitive level, psychopaths demonstrate impairments in executive functioning (premeditation and perseverance) and response modulation (see above) when these processes are mediated by orbital / ventrolateral cortex but unimpaired when mediated by dorsolateral prefrontal cortex. Furthermore, the amygdala dysfunction results in impairments in the formation of stimulus-reinforcement associations. Psychopaths are particularly known for their inability to make aversive stimulus-reinforcement associations (Blair, 2004). Since the distress of others can be seen as one class of aversive stimuli, this is in line with research results indicating a fear system dysfunction in psychopathy. It is obvious that these cognitive impairments interfere with a child's socialization. In this light, it is interesting to note that ineffective parenting strategies were associated with an increased number of conduct problems in children without psychopathic traits but not in children with psychopathic traits (Oxford, Cavell, \& Hughes, 2003; Wootton, Frick, Shelton, $\&$ Silverthorn, 1997). In addition, psychopaths also show impairment in their ability to alter stimulus-response associations as a function of contingency change. That is, psychopaths have trouble adjusting their behavior when an action that used to be rewarding, now leads to punishment. Blair et al. (2006) postulated that this impairment places psychopaths at a heightened risk for frustration and, consequentially, frustration-based aggression. At the behavioral level, these cognitive impairments are thought to disrupt the child's ability to be socialized and therefore put the child at risk for learning to use antisocial behavior. More research needs to be conducted in order to provide an understanding of psychopathy at the molecular and genetic level. In the meantime, the model proposed by Blair and colleagues (2006) provides a promising integration of the experimental psychopathy literature and provides a theoretical framework which can guide future research into the etiology of the disorder. 


\section{Stability of psychopathy from childhood into young adulthood}

Initial estimates of the stability of psychopathic traits were provided by Frick, Kimonis, Dandreaux and Farell (2003). They demonstrated a substantial degree of stability in parent ratings of psychopathic traits as measured by the APSD over a 4-year period in a sample of non-referred children. However, a trend could be demonstrated that some youth high on psychopathic traits showed less severe levels of these traits over time. The most consistent predictors of the stability of psychopathic traits included the child's level of conduct problems, the socioeconomic status of the child's family, and the quality of parenting. Blonigen et al. (2006) provided support for the stability of the interpersonal-affective features of psychopathy from late adolescence into early adulthood, whereas the social deviance features were found to decline over this developmental period.

The most comprehensive study into the stability of psychopathic traits to date has been performed by Lynam, Caspi, Moffit, Loeber and StouthamerLoeber (2007) who examined the relation between psychopathy assessed at age 10-13 by using the mother-reported CPS and psychopathy assessed at age 2226 by using the interviewer-rated Psychopathy Checklist: Screening Version (PCL:SV; Hart, Cox, \& Hare, 1995). Participants $(N=316)$ were members of the middle sample (when children were age 10) of the Pittsburgh Youth Study, consisting of boys attending the fourth grade in the public school system. An initial screening assessment had identified nonhigh-risk participants and high-risk participants, who demonstrated the most severe disruptive behavior problems. Psychopathy was found to be moderately stable $(r=.31)$ and this relation was present for the PCL:SV total score and for the four dimensions. It should be noted, however, that CPS total scores at age 10-13 were more strongly related to the lifestyle and antisocial dimensions at age 22-26 than to the interpersonal and affective dimensions. Most importantly, the stability of psychopathy scores was found to be the same across levels of initial risk status and initial psychopathy level. Furthermore, these relations survived controls for 13 different constructs, including race, family structure, family socioeconomic status (SES), neighborhood SES, measures of parenting, peer 
delinquency, previous delinquency, and measures of behavioral impulsivity, cognitive impulsivity, and verbal IQ. Regarding diagnostic accuracy, the results demonstrated good specificity (i.e., the conditional probability that an individual was low in psychopathy at age 13 given that he was not psychopathic at age 24 and negative predictive power (i.e., the conditional probability that an individual was not psychopathic at age 24 given that he was not psychopathic at age 13, whereas positive predictive power was poor (i.e., the conditional probability that an individual was psychopathic at age 24 given that he was psychopathic at age 13). The authors suggested that these results may be expected given the relatively low base rate of psychopathy in the sample. Overall, stability estimates are quite similar to what is typically found for measures of personality in adolescent and adult samples, suggesting that adolescent psychopathy is a developmental precursor to adult psychopathy. However, future research should examine whether these findings are generalizable to other developmental phases. Finally, Salekin (2008) recently demonstrated that psychopathic traits in early adolescence are predictive of general and violent recidivism in early adulthood. This relationship was significant even after controlling for 14 variables theoretically linked to offending. 
Intermezzo: Case Vignette (2)

In the first two weeks of his stay in the semi-secure living group, Harry made a charming and friendly impression (item 1). He was polite, consulted the treatment staff when making decisions, and kept all agreements. Harry was an eloquent speaker (item 1) and made the impression that he knew what he wanted for the future (item 13). However, taking into account his poor results in school, and his lack of perseverance (item 13), his plans seemed unrealistic: he wanted to run his own restaurant. When the treatment staff confronted him with this, he discarded their arguments by calling them 'rubbish'. At the same time, however, he had no concrete plans on how to achieve his goals (item 13). A profound lack of perseverance and realistic goals also became evident when Harry quit his education after just one month and was not capable of keeping a job (item 13). When he didn't feel like going to work, he called in sick or didn't show up.

In his associations with the other boys in the living group, Harry made an arrogant impression. He boasted about his qualities, although his ideas did not seem to fit reality (item 1). He was often condescending towards the other boys and he thought he was smarter (item 1).
Harry often told improbable stories and made inaccurate statements that, when asked about, were defended by him by using vague statements (item 4 ). When he was confronted with a lie, Harry gave another turn to the story to make it sound credible (item 4). In conversations, Harry adjusted his use of language to the person he was talking to (item 1). For instance, he used more sophisticated language when speaking to older people. However, he often used words and terms in the wrong context or pronounced them incorrectly (item 1). After a few months in the new living group, it became apparent that Harry did not obey the rules. He left unauthorized several times (item 19). Gradually, the treatment staff became informed about his lying, cheating and manipulative behavior towards the other boys (item $4 \& 5$ ). The other boys were scared of Harry, and Harry abused this to manipulate them for personal gain (item 5), for instance, by making a boy steal and buy marihuana for him. In addition, Harry was suspected of extorting the more vulnerable boys in the living group (item 20) or refusing to return money he borrowed from them (item 5; item 9). When confronted with this, Harry believed the institution was responsible for paying his increasing number of debts (item 16). Harry also started using 
alcohol and drugs again (item 3) and demonstrated problematic behavior during his authorized leaves. Although he said he loved his family, he cheated and stole from them and threatened his mother and brother (item $7 \&$ 8). When his mother annoyed him, he broke off all contact with her without hesitation or remorse (item $7 \&$ 8). Harry had a girlfriend and said he loved her very much and couldn't live without her. At the same time, however, he manipulated her and forced his will upon her (item 5 \& 17). She had to conform to his rigid values about what their relationship should look like; if she didn't, Harry threatened to break off the relationship (item 7). Harry didn't have a stable circle of friends but associated with a continuously changing group of acquaintances (item 17). When treatment staff confronted Harry with his problematic behaviors, he made up stories and tried to escape from negative consequences (item $4,6,16$ ). In addition, he placed the blame on others (item 16) and reacted in a verbally or physically aggressive fashion (item 10). When Harry was in the open setting for about half a year, the treatment staff decided that he had to be transferred back to the secure setting (item 19). Although there was no treatment progress, Harry was eventually sent on probationary leave because the end of his mandatory treatment order (and all possible forms of supervision) was nearing. 


\section{Ethical and methodological issues}

Ethical and methodological issues accompanying the growing body of research on psychopathy in youth have been discussed by several authors (e.g., Frick, 2000; Edens, Skeem, Cruise, \& Cauffman, 2001; Seagrave \& Grisso, 2002). First, the DSM-IV (American Psychiatric Association, 1994) requires a person to be 18 before a diagnosis of a personality disorder is made. A diagnosis of psychopathy before the age of 18 is conflicting with this criterion (Hart, Watt, \& Vincent, 2002).

Second, the term psychopathy is generally considered to be pejorative since it is associated with an aura of dangerousness and untreatability. A diagnosis of psychopathy may therefore have great impact when used in clinical decision-making in adolescent forensic psychiatric practice. For example, because of this diagnosis, an adolescent may be transferred to adult court and to sentencing as an adult or be denied treatment (Seagrave $\&$ Grisso, 2002). The association between psychopathy and untreatability has not been supported by empirical evidence. Caldwell, Skeem, Salekin and Van Rybroek (2006) provided initial evidence for the effectiveness of treatment in adolescents with psychopathic traits. They compared recidivism rates of juvenile offenders high on psychopathic traits who received treatment as usual in a conventional juvenile correctional institution to those who received an intensive treatment program. The treatment programs differed in the available resources (e.g., number of available psychologists and psychiatrists per youth), and program philosophy. That is, in the treatment as usual condition, sanctioning was used as a means of holding youth accountable for their own actions, whereas in the intensive treatment condition the correctional philosophy focused on replacing antagonistic bonds by conventional bonds. Results demonstrated that psychopathic adolescents in the treatment as usual condition were more than twice as likely to recidivate in a violent way in the community during a 2-year follow-up than adolescents who had received intensive treatment.

Third, there is ongoing academic controversy about whether psychopathy is dimensional or categorical in nature. While some researchers assert that 
there are fundamental differences between psychopaths and nonpsychopaths (Harris, Rice, \& Quinsey, 1994), others failed to demonstrate evidence for a latent taxon underlying psychopathy in adults (Edens, Marcus, Lilienfeld, \& Poythress, 2006) and in youth (Murrie, Marcus, Douglas, Lee, Salekin, \& Vincent, 2007). It has been suggested that psychopathy may in fact be a constellation of extreme levels of continuously distributed personality dimensions (Benning, Patrick, Blonigen, Hicks, \& Iacono, 2005).

Fourth, it is argued that usage of the term psychopathy for youth is not appropriate as long as research into its stability is still limited (Seagrave \& Grisso, 2002). Additionally, Hart et al. (2002) referred to three developmental psychopathology principles, including heterotypic continuity (the same trait manifested in different ways across developmental stages), equifinality (diverse developmental pathways may lead to the same outcome in adulthood), and multifinality (the same developmental pathway may lead to diverse outcomes). These principles suggest that even though psychopathy may exist in childhood or adolescence, it may look very different from psychopathy in adulthood. A related methodological issue is that behaviors and attitudes similar to operational definitions of adolescent psychopathy, for instance rapid changes in peer relationships and risk taking behavior, may also be due to the transient developmental process of adolescence. This increases the possibility of 'false positives'- a diagnosis of psychopathy when psychopathy is, in fact, not present (Seagrave \& Grisso, 2002).

Although each one of these issues should be taken seriously, not using the term 'psychopathy' or 'psychopathic traits' would ignore the fact that good evidence indicates these traits do exist in youth. In addition, the importance of early identification of youth with psychopathic traits in order to enhance interventions early in life, is a critical argument in favor of usage of the term psychopathy. Several authors, while acknowledging the great potential of applying the concept of psychopathy to youth, have made recommendations on how to use it cautiously and judiciously by taking into account the above ethical and methodological considerations. For instance, Farrington (2005) has proposed that focusing on child and adolescent 
psychopathy as defined by its underlying dimensions (i.e., interpersonal, affective, lifestyle, and antisocial) avoids to some extent the stigmatizing effects of labeling youth as psychopaths. Furthermore, Johnstone and Cooke (2004) suggested that developmentally informed conceptualizations of psychopathy dimensions should be used to measure psychopathy, in order to avoid false positive or false negatives that can be caused by transient psychopathic-like behaviors. Also, Vitacco and Vincent (2006) recently proposed seven recommendations, which are meant to serve as heuristics in guiding clinicians in the ethical practice of assessing and treating antisocial youth. These recommendations include: 1) avoid diagnostic labeling, 2) conduct comprehensive assessments of risk, 3) consider protective factors, 4) conduct multiple assessments over time, 5) consider environmental influences, 6) target psychopathic traits in treatment, and 7) be aware of inappropriate uses of psychopathy assessments. Finally, researchers have advocated that psychopathy instruments like the PCL:YV should be the focus of intense psychometric scrutiny (Petrila \& Skeem, 2003; Skeem \& Cauffman, 2003). The studies in the present thesis were designed with this purpose in mind.

\section{Setting of the present studies}

Jongerenhuis Harreveld is one of the thirteen juvenile justice treatment institutions in The Netherlands. The research reported in this thesis was conducted in the two secure divisions and in one semi-secure division of Jongerenhuis Harreveld. At the time of this research project, treatment was provided for 100 boys and 80 girls in these divisions

The boys and girls had been sentenced by court to a supervision order (OnderToezichtStelling; OTS) or a mandatory treatment order (Plaatsing in een Inrichting voor Jeugdigen; PIJ). The duration of a mandatory treatment order can vary from two to six years, depending on the seriousness of the offense, on whether a mental disorder was present at the time of the offense, and on whether the parenting situation was seriously compromised. A supervision order is a civil measure, which can be imposed when a child's development is psychologically or physically threatened because of 
incompetent parenting and/or behavioral problems in the child. During the supervision order, the custody of the adolescent is the shared responsibility of both the parents and the child care agency.

A central goal of Jongerenhuis Harreveld is to provide the adolescent with appropriate education and treatment, in order to increase his/her future ability to function adaptively within society. Differentiated treatment is provided, that is, for every subdivision a specialized treatment method is developed to address the particular treatment needs of the target group. Target groups at the time of the project included: sex offenders whose offenses are characterized by an obsessive-compulsive aspect, sex offenders whose offenses are characterized by an opportunistic aspect, seriously antisocial and / or aggressive adolescents with attachment problems, antisocial adolescents with serious mental disorders, and antisocial adolescents with substance abuse problems. Adolescents reside in a living group of 10 boys or girls, where they can develop and practice more adequate skills to cope with their risks, needs, limitations and possibilities. They are supervised by group leaders who help provide the structure of daily life. In the first six weeks after admission, a treatment plan is constructed. In this plan, individual treatment goals are specified, as well as the means to address these goals. Besides treatment in the group, adolescents participate in various treatment modules, including Aggression Replacement Training (ART; Glick \& Goldstein, 1987), social skills training, special programs for substance abusers or sex offenders; and individual psychotherapy. Treatment progress is evaluated every three months by the treatment team, the adolescents' parents, and child protection worker (in the case of a supervision order). Finally, in light of the remedial purposes of admission to Jongerenhuis Harreveld, education has a vital role.

The goal of forensic psychological assessment during treatment in Jongerenhuis Harreveld is twofold. First, results from personality assessment are used as input for formulation of the treatment plan. Second, repeated assessments are used to provide information on the adolescent's treatment progress and whether the mandatory treatment order should be 
extended. A test battery was selected to cover several domains of functioning, including personality, emotion, relationships, sexuality, skills, coping, and risk of future offending. Table 4 presents the domains and the instruments used to assess them. This overview does not include the PCL:YV since it was administered for research purposes.

\section{Aims of the present thesis}

1. To examine the reliability and validity of the Dutch Psychopathy Checklist: Youth Version (PCL:YV) as a measure of psychopathic traits in Dutch male adolescents in a juvenile justice treatment institution (Chapter 2). 2. To examine the reliability and validity of the Dutch PCL:YV as a measure of psychopathic traits in female adolescents in a juvenile justice treatment institution and to explore whether there are gender-specific issues that have to be considered when assessing psychopathic traits in female adolescents (Chapter 3).

3. To explore which symptoms are most important in the assessment of psychopathy in Dutch adolescent offenders and whether the PCL:YV measures psychopathic traits in the same way in adolescents as the Psychopathy Checklist-Revised does in Dutch adult offenders (Chapter 4).

4. To establish the predictive validity of the Dutch PCL:YV for institutional infractions (Chapter 5).

5. To explore whether the Dutch Youth Psychopathic traits Inventory (YPI) can be used as a reliable and valid measure for the identification of psychopathic traits in the general Dutch adolescent population (Chapter 6).

In all studies of the present thesis, special attention was directed at the value of the separate psychopathy dimensions in order to gain more insight into their individual clinical utility. 
TABLE 4

PSYCHOLOGICAL TEST BATTERY USED AT JONGERENHUIS HARREVELD

AT THE TIME OF THESE STUDIES

Domain Instruments

Personality

Emotion

Relationhips

Sexuality

Skills

Coping

Risk of future violence
MMPI-a(or -2) or NPV-J, SVPA

MMPI-a (or -2) or NPV-J, SVPA

MMPI-a (or -2), ICL-R, SVPA, TVA

SVPA, TVA

SVPA, TVA

UCL(-A)

SAVRY; SVR-20

Note. ICL-R = Interpersonal Checklist-Revised (De Jong, Van den Brink, \& Jansma, 2000); MMPI-a (or -2) = Minnesota Multiphasic Personality Inventory-adolescent version (Van Dijk, Cornelissen, \& Derksen, 2000) or adult version (Derksen, De Mey, Sloore, \& Hellenbosch, 1995); NPV-J = Junior Nederlandse Persoonlijkheids Vragenlijst (Junior Dutch Personality Questionnaire; Luteijn, Van Dijk, \& Van der Ploeg, 1989); SAVRY = Structured Assessment of Violence Risk in Youth (Lodewijks, Doreleijers, De Ruiter, \& De Wit-Grouls, 2001); SVPA = Seksualiteits Vragenlijst Pakket voor Adolescenten (Sexuality Assessment Package for Adolescents; Bullens \& Hendriks, 1999); SVR-20 = Sexual Violence Risk-20 (Hildebrand, De Ruiter, \& Van Beek, 2001); TVA = vragenlijst Taken en Vaardigheden van Adolescenten (Questionnaire of responsibilities and skills in adolescents; Slot \& Spanjaard, 1999); UCL = Utrechtse Coping Lijst (Coping Skills Questionnaire; Schreurs, Van de Willige, Tellegen, \& Brosschot, 1988) 
CHAPTER 1 


\section{SUMMARY}

The present study examined the reliability and construct validity of the Dutch version of the Psychopathy Check List:Youth Version in a sample of male adolescents admitted to a secure juvenile justice treatment institution $(\mathrm{N}=98)$. Hare's (2003) four-factor model was used to examine reliability and validity of the separate dimensions of psychopathy. Interrater reliabilities were good to excellent for the PCL:YV total score and most factor scores, except for the affective factor. Several suggestions are offered for optimizing reliability of this factor. Finally, meaningful associations between PCL: YV scores and scores on the Minnesota Multiphasic Personality InventoryAdolescent (MMPI-A) and the Interpersonal Checklist-Revised (ICL-R) support the construct validity of the PCL:YV total score as well as the four factors in the Dutch context.

\section{Introduction}

The assessment of psychopathic traits in adolescents has become an important aspect of forensic psychological assessment, with potentially grave implications for sentencing, diversion practice and treatment (Petrila \& Skeem, 2003; Skeem \& Cauffman, 2003). In adult male offenders, the concept of psychopathy is associated with an early onset of antisocial behavior (Forth \& Burke, 1998; Hare, Hart, Forth, Harpur, \& Williamson, 1998), deviant performance on neurocognitive tasks (e.g., Newman \& Schmitt, 1998; Levenston, Patrick, Bradley, \& Lang, 2000), higher recidivism rate (Gendreau, Goggin, \& Smith, 2002; Hildebrand, de Ruiter, \& de Vogel, 2004; Walters, 2003b), and limited response to treatment (Ogloff, Wong, \& Greenwood, 1990; Rice, Harris, \& Cormier, 1992; Seto \& Barbaree, 1999). However, positive effects of treatment on the violent and criminal behaviors of adults and adolescents with psychopathic traits have also been demonstrated (Caldwell, Skeem, Salekin, \& van Rybroek, 2006; Skeem, Monahan, \& Mulvey, 2002).

Several scholars have argued that the identification of psychopathic traits in childhood or adolescence may offer a better understanding of the etiology 
of the disorder and may provide starting-points for targeting interventions (Forth, Hart, \& Hare, 1990; Forth \& Mailloux, 2000; Frick, Bodin, \& Barry, 2000). For this purpose, the Psychopathy Checklist-Revised (PCL-R; Hare, 1991, 2003), the most widely validated measure for the assessment of psychopathy in adults, has been extended downward for use with adolescents.

The Psychopathy Checklist: Youth Version (PCL:YV; Forth, Kosson \& Hare, 2003) closely resembles the PCL-R, but item descriptions were modified to take into account the restricted life experience of the adolescent and to emphasize peer contacts instead of romantic relationships (Forth et al., 2003). Similar to the PCL-R, the items of the PCL:YV are scored on a three-point rating scale $(0=$ item does not apply, 1 = item applies to a certain extent, 2 $=$ item definitely applies), resulting in a dimensional total score ranging from 0 to 40 . In contrast to the PCL-R, there is no designated cut-off score for the PCL:YV. Scores should be interpreted as dimensional ratings of the degree to which an adolescent matches the prototypical psychopath (Forth et al., 2003).

The factor model underlying the PCL:YV is still under investigation. While early studies suggested a similar two factor-structure as the one traditionally thought to underlie adult psychopathy (e.g., Brandt, Kennedy, Patrick, \& Curtin, 1997; Forth \& Mailloux, 2000), the PCL:YV manual (Forth et al., 2003) provides evidence of two alternative models that have been proposed for the PCL-R: a modified version of the hierarchical three-factor model (Cooke \& Michie, 2001) and a parceled four-factor model (Hare, 2003). In both models, the original Factor 1 (Hare, 1991) is divided into an interpersonal dimension (new Factor 1) and an affective dimension (new Factor 2). A new Factor 3 comprises impulsive behavioral traits and an irresponsible lifestyle. The Hare (2003) four-factor model includes an additional fourth factor comprising antisocial behaviors. Several studies have demonstrated both models to have generally good fit in male adolescents. The four-factor model was more parsimonious from a mathematical modeling perspective (Jones, Caufmann, Miller, \& Mulvey, 2006; Neumann, Kosson, Forth, \& Hare, 2006; Salekin, Brannen, Zalot, Leistico, \& Neumann, 2006), whereas the three-factor model is considered to be more theoretically coherent (e.g., Cooke \& Michie, 
2001; Cooke, Michie, Hart, \& Clark, 2004). In fact, other child and adolescent psychopathy measures, such as the Antisocial Process Screening Device (APSD; Frick \& Hare, 2001) and the Youth Psychopathic traits Inventory (YPI; Andershed, Kerr, Stattin, \& Levander, 2001), do not (YPI) or only scarcely include antisocial items (APSD; one item).

Critics have argued that the use of a simple downward extension of adult psychopathy measures such as the PCL-R may not be warranted because of several methodological and ethical problems (Hart, Watt, \& Vincent, 2002; Seagrave \& Grisso, 2002). One concern is that psychopathy may not be manifested in the same way across the lifespan. More specifically, behaviors and attitudes similar to operational definitions of adolescent psychopathy, for instance rapid changes in peer relationships and risk taking behavior, may in fact be caused by the transient developmental process of adolescence. This increases the possibility of 'false positives'- a diagnosis of psychopathy when psychopathy is, in fact, not present. The negative connotations associated with the term psychopathy (e.g., dangerousness, untreatability) necessitate that high psychometric standards be met before using psychopathy measures in clinical forensic practice. Careful scrutiny of the psychometric properties of the PCL:YV in the past decade, has provided accumulating evidence of the reliability and construct validity of $\mathrm{PCL}: \mathrm{YV}$ ratings, which are briefly summarized below.

\section{Evidence of reliability}

Good interrater reliability and internal consistency of the PCL:YV total score has been demonstrated in several studies (e.g., Brandt et al., 1997; Kosson, Cyterski, Steuerwald, Neumann, \& Walker-Matthews, 2002; O’Neill, Lidz, \& Heilbrun, 2003; Skeem \& Cauffman, 2003). Campbell, Pulos, Hogan, and Murry (2005) performed a meta-analysis of 28 independent adolescent samples, to examine the reliability of the PCL:YV and related instruments (i.e., PCL-R, modified versions of the PCL-R, and the PCL: Screening Version; Hart, Cox, \& Hare, 1995). The interrater reliability and internal reliability of the total score were good (.91 and .85, respectively). However, several factors 
were found to affect reliability. For instance, interrater reliability was found to be better in samples with greater PCL:YV total score variability, whereas samples with a large number of subjects with uniformly low or high PCL: YV scores were more difficult for raters to agree upon. Furthermore, the use of the PCL-R in adolescent samples led to better internal consistency than the use of the PCL:YV, suggesting that the modifications associated with the PCL:YV have introduced greater heterogeneity in scoring.

\section{Evidence of construct validity}

The concept of the nomological network (Cronbach \& Meehl, 1955) provides an appealing theoretical framework for understanding the construct validity of psychopathy. In this framework, constructs are continually redefined and clarified through their relationships with other constructs. A first layer of the nomological network surrounding psychopathy involves the interrelationship between various measures of psychopathy. The construct validity is supported when these measures strongly relate to each other. However, limited convergence has been demonstrated between the PCL:YV and other measures of psychopathic traits in adolescence (Lee, Vincent, Hart, \& Corrado, 2003; Murrie \& Cornell, 2002; Salekin, Leistico, Trobst, Schrum, \& Lochman, 2005; Skeem \& Cauffman, 2003). A method effect (self report versus clinician ratings) was demonstrated to underlie this lack of convergence (Lee et al., 2003), suggesting that these different methods do not capture the concept in the same manner.

A second layer in the nomological network is the relationship of psychopathy measures with measures of other personality disorders. Construct validity is supported when psychopathy measures are strongly associated with related disorders, while demonstrating divergent relationships with unrelated disorders. Psychopathic traits as measured by the PCL:YV are related to an early onset of antisocial behavior and generally have a greater number and variety of Conduct Disorder (CD) symptoms and more severe CD symptoms than boys without these traits (Forth et al., 1990; Kosson et al., 2002; Myers, Burket, \& Harris, 1995; Rogers, Johansen, Chang, 
\& Salekin, 1997).

A third layer of the nomological network involves the relationship with psychopathy measures with normal-range personality dimensions. Construct validity is supported when psychopathy measures demonstrate theoretically meaningful relationships with personality dimensions. Indeed, several researchers have demonstrated that the construct of adolescent psychopathy could be meaningfully placed into a personality framework. For instance, Brandt et al. (1997) demonstrated that PCL:YV scores were related to the clinical scales 4 and 9 (reflecting amoral rebelliousness and impulsive tendencies) of the Minnesota Multiphasic Personality Inventory-Adolescent version (MMPI-A). Furthermore, psychopathic traits were found to be related to the Big Five factors Agreeableness and Conscientiousness (Salekin et al., 2005), and to an emotionally cold (Salekin et al., 2005) and dominant (Forth, Brown, Hart, \& Hare, 1996) interpersonal style. However, some findings have been equivocal. For instance, while a lack of anxiety and negative affectivity is conceptually supposed to be a central feature of psychopathy (for a review, see Skeem, Mulvey, \& Grisso, 2003), PCL:YV scores were not (Brandt et al., 1997; Skeem \& Cauffman, 2003) or even positively associated with negative affect (Kosson et al., 2002). However, in order to determine whether this finding makes theoretical sense, the unique relations between the separate psychopathy dimensions and anxiety should be examined. Specifically, the interpersonal or affective dimension would be expected to correlate negatively with anxiety, whereas the lifestyle and behavioral features have been demonstrated to have a positive relationship with negative affect (e.g., Frick, Lilienfeld, Ellis, Loney, Silverthorn, 1999).

Because increased importance is placed on the separate psychopathy dimensions, recent research has focused on evaluating their construct validity by comparing each dimension with external criteria (Farrington, 2005). For instance, Hall, Benning and Patrick (2004) examined the associations of the four PCL-R factors with personality, behavior, and adaptive functioning in adult male offenders. The interpersonal psychopathy dimension was found to be related to social dominance, low stress reactivity, and increased adaptive 
functioning. Furthermore, the affective factor was related to low social closeness and violent offending, and the lifestyle factor was associated with negative emotionality, disinhibition, reactive aggression, and poor adaptive functioning. The antisocial dimension generally demonstrated similar correlations as the lifestyle dimension, but had greater positive correlations with assault charges and overall number of violent charges. Differential associations between Hare's traditional two psychopathy factors and external criteria have also been demonstrated in adolescent samples (Lynam, Caspi, Moffitt, Raine, Loeber, \& Stouthamer-Loeber, 2005). However, the new factor models proposed by Cooke and Michie (2001) and Hare (2003) have not yet been tested on their construct validity in youth.

\section{The present study}

Since prior studies regarding the psychometric properties of the PCL:YV are almost exclusively based on North-American samples, cross-cultural validation seems a logical next step. The implementation of the PCL:YV in a new crosscultural context should be accompanied by an evaluation of the psychometric status of the instrument in that particular context. Therefore, the first aim of the present study is to examine the interrater and internal reliability (item homogeneity and internal consistency) of the Dutch language version of the PCL:YV (Psychopathie Checklist: Jeugd Versie; PCL:JV; de Ruiter; Kuin, de Vries \& Das, 2002) in a sample of 98 male adolescents in a juvenile justice treatment institution.

The second aim is to explore the construct validity of the Dutch PCL:YV by relating psychopathy total and factor scores to scores on the Dutch Minnesota MMPI-A (Butcher et al., 1992; Dutch version: van Dijk, Cornelissen, \& Derksen, 2000). In addition, PCL:YV scores are related to scores on the Interpersonal Checklist (ICL-R; LaForge \& Suczek, 1955; Dutch version: de Jong, van den Brink, \& Jansma, 2000). Based on previous findings, PCL:YV total scores are expected to be positively associated with: - (antisocial) externalizing behavior (MMPI-A clinical scale 4; subscale Pd2; content scales conduct problems, school problems, anger); 
- substance abuse problems (MMPI-A supplementary scales MAC-R, PRO and ACK);

- impulsivity (MMPI-A clinical scale 9; subscales Sc3, Sc4, Sc5);

- dominant and hostile interpersonal style (ICL-R dominance vector), and inversely related to:

- social introversion (MMPI-A clinical scale 0; subscales Si1, Si2, Hy1; content scale social discomfort);

- submissive and friendly interpersonal style (ICL-R affiliation vector).

The third aim of the study is to examine the construct validity of Hare's four-factor model (2003). Psychopathy dimensions are expected to be differentially associated with external correlates. More specifically, the interpersonal dimension is expected to be primarily inversely associated with scales measuring social introversion, and a submissive and friendly interpersonal style. In addition, this dimension is expected to be primarily positively related to a dominant interpersonal style. The lifestyle and antisocial dimensions are expected to be most strongly related to scales measuring externalizing behavior, substance abuse and impulsivity. Although the PCL:YV total score is expected to be unrelated to anxiety, the affective psychopathy dimension is expected to show a significant inverse relation to anxiety (MMPI-A content scale ANX).

There is reason to believe that the psychological characteristics of sexual offenders are somewhat different from the psychological characteristics of other offenders (e.g., less extravert and impulsive, more neurotic; see for a review Van Wijk et al., 2006). In a mixed offender sample such as the present one, this may affect the relations of the PCL:YV to the selected MMPI-A and ICL-R scales. Therefore, the final aim of the study is to examine whether the associations of the PCL:YV with external correlates are robust across offender types (sexual vs. non-sexual offenders). 


\section{METHOD}

\section{Participants}

The study sample comprised male adolescents admitted to a secure section of Jongerenhuis Harreveld, a juvenile justice treatment institution. Jongerenhuis Harreveld admits juveniles from all over The Netherlands and is specialized in the treatment of juvenile sex offenders and mentally disordered juvenile offenders. This results in the admission of only the most severely disordered boys. Boys are either sentenced to a supervision order or a mandatory treatment order by the court. The duration of a mandatory treatment order varies from two to six years, depending on the seriousness of the offense, on whether a mental disorder was present at the time of the offense and on whether the parenting situation was seriously compromised. A supervision order is a civil measure, which can be imposed when a child's development is threatened because of incompetent parenting and/or behavioral problems of the child. During the supervision order, the parents and a legal guardian share the responsibility of the adolescent's custody.

From January 2002 until December 2004, every newly admitted boy was asked to participate in the study. Written informed consent was obtained from almost all recruited boys (response rate: $90.7 \% ; N=114$ ). Furthermore, their parents were informed about the purpose and procedure of the study, and were given the opportunity to object to participation (passive informed consent). The PCL:YV was administered at the conclusion of treatment to a small subset of boys $(10.3 \% ; n=13)$. Since the PCL:YV was designed for adolescents between the ages 11 and 18 (Forth et al., 2003), twenty-nine boys $(23 \%)$ were removed because they were 19 years or older when the PCL:YV was coded. At admission, the mean age of the boys in the final sample $(N=$ 98) was 16.0 years $(S D=1.3$; range $=13-18)$. In terms of ethnic origin, $72 \%$ was Caucasian-European, $7 \%$ was Surinamese, 5\% was Moroccan, 3\% was from the Netherlands Antilles, and $13 \%$ had another ethnic background. Ninety-four percent of the boys were placed in the institution in accordance with a mandatory treatment order, and $51 \%$ in accordance with a supervision 
order. Of the boys with a mandatory treatment order, $36 \%$ had been convicted for a violent offense, $59 \%$ for a sexual offense, $2 \%$ for a property offense, and $2 \%$ for arson. Although boys with a supervision order were not convicted for any criminal offense, they were all suffering from serious (antisocial) behavior problems requiring residential treatment in a secure facility.

\section{Instruments}

Psychopathy Checklist: Youth Version

PCL:YV ratings were based on the administration of the Dutch translation (Vertommen, Verheul, De Ruiter and Hildebrand, 2002) of the semi-structured PCL-R interview designed by Hare (1991) together with collateral information available for each adolescent upon admission. This information included police files, psychiatric and psychological evaluations, and observational reports from previous institutional care. Each subject was rated by two independent raters. For the items 9 (Parasitic orientation), 11 (Impersonal sexual behavior), 13 (Lacks goals), 17 (Unstable interpersonal relationships), and 18 (Serious criminal behavior), the descriptive criteria were slightly revised from the Canadian original. Revisions comprised clarifications on when the level of psychopathic symptoms becomes non-normative. For example, because a certain lack of commitment to long term goals (item 13) is a common part of adolescence, raters were advised to also take into account whether the adolescent had demonstrated commitment to more short term goals. Clarifications were added to the item description in separate textboxes in the Manual (see Table 1 for an overview of these revisions). Dr. Adelle Forth, first author of the Psychopathy Checklist: Youth Version (Forth et al., 2003), was informed of these revisions. 
The four-factor model (Hare, 2003), comprising an Interpersonal (Factor 1), Affective (Factor 2), Lifestyle (Factor 3), and Antisocial (Factor 4) dimension, was used for the examination of the reliability and construct validity of the separate psychopathy dimensions.

TABLE 1

REVISIONS OF ITEM DESCRIPTIONS

PCL:YV-item

Revised item description

Item 9: Parasitic orientation

Item 11: Impersonal sexual behavior

Item 13: Lacks goals

Item 17: Unstable interpersonal relationships

Item 18: Serious criminal behavior
A score of 1 can be given in case the adolescent uses criminal activity to provide financially for himself or does not have any clear means of financial support. For scoring this item, emphasis is placed on infidelity, voluntary prostitution, extremely high frequency of impersonal sexual relationships ('players') and / or a great variety of sexual activity.

Takes into account whether the adolescent has demonstrated to be committed to short term goals.

A score of 1 can be given when there is no clear sign of instability of the relationships, but the relationships can be characterized as quite superficial.

The item description was replaced by the item description of this item in the PCL-R.

Minnesota Multiphasic Personality Inventory-adolescent version In this study, the MMPI-A (Butcher et al., 1992; authorized Dutch version: Van Dijk et al., 2000) was used. Dutch norms are available for adolescents 13 to 19 
years of age. The MMPI-A is a 478-item self-report questionnaire, which offers insight into psychopathology and personality traits. The instrument consists of a number of validity scales, which allow insight into the response style of the subject (e.g., socially desirable or inconsistent responding). Further, nine clinical scales, 15 content scales and six supplementary scales provide information on the presence or absence of a variety of psychopathological symptoms. A study of the MMPI-A in a Dutch general population sample of 1182 adolescents showed that American adolescents score significantly higher on most MMPI-A scales (Derksen, Van Dijk, \& Cornelissen, 2003). The oneweek test-retest reliability is reasonable and the internal consistency of most of the scales is adequate, except for two clinical scales [Scales 3 (Hysteria) and 5 (Masculinity/femininity)], one content scale (low ambition) and one supplementary scale (MacAndrew Alcoholism; Derksen et al., 2003). For the present study, MMPI-A profiles were considered invalid when scores on the TRIN and/or VRIN scales were over 75. Ninety-two percent of the boys completed the MMPI, and all but four of these boys produced a valid profile (internal consistency of total score: $\alpha=.90$ ).

\section{Interpersonal Checklist-Revised}

The authorized Dutch translation of the Interpersonal CheckList-Revised (ICL-R; de Jong et al., 2000) was used to map interpersonal behavioral styles. The ICL- $R$ is a renewed version of the Interpersonal CheckList (LaForge \& Suczek, 1955) and based on Leary's (1957) model of interpersonal behavior. The ICL-R consists of 160 items, which are rated yes or no. The instrument contains ten behavioral dimensions: PA (managerial-autocratic), BC (competitive-exploitive), DE (aggressive-blunt), FG (distrustful-skeptical), nFnG (reserved-aloof), HI (modest-self-efficacing), JK (docile-dependent), LM (cooperative-overconventional), $\mathrm{NO}$ (responsible-overgenerous), and $\mathrm{nNnO}$ (extravert-gregarious). The scores on the ten dimensions can be transformed into a vector score, which indicates whether the interpersonal style is predominantly characterized by dominance versus submissiveness, and by hostility versus friendliness. The ICL-R manual provides information on the 
psychometric properties of the ICL-R in Dutch samples. The intercorrelations and the circumplex analysis support the hypothesized circular arrangement of the interpersonal styles. The test-retest reliability of the interpersonal styles is moderate to good (ICC's range from .57 to .83; de Jong et al., 2000).

ICL- $R$ ratings were obtained from the boys themselves as well as from group leaders who acted as the adolescent's designated mentor during their stay in the institution. The response rates were $76 \%$ for the group leaders (internal consistency of total score: $\alpha=.76$ ) and $90 \%$ for the boys (internal consistency of total score: $\alpha=.91$ ).

\section{Procedure}

All PCL raters $(N=12)$ worked as mental health professionals at Jongerenhuis Harreveld and received training in the administration and scoring of the PCL-R/ PCL:YV by the second author (Corine de Ruiter, Ph.D.) and/or Pascalle van der Wolf, M.Sc., who were both trained by Drs. Robert D. Hare and David J. Cooke. The training included a review of the clinical construct of psychopathy and the research literature pertaining to it, and practice scoring using videotapes of two Dutch adult forensic psychiatric patients and three adolescent offenders. After the coding of the PCL:YV by two independent raters, a meeting was planned to obtain a final (consensus) rating for the adolescent. This procedure was chosen in order to maximize information exchange and to facilitate scoring accuracy. In most cases, the supervising psychologist of the ward conducted the PCL interview and the PCL:YV rating. This procedure was chosen for several reasons. In the first place, the interview was the first occasion for extended contact and provided an opportunity for rapport building with the adolescent. Secondly, we believe it is important to investigate whether the PCL:YV can be reliably coded by practicing clinicians since this partially determines its ecological validity.

The administration of the MMPI-A and the ICL-R took place in groups of three adolescents at a time, supervised by a psychologist or a research assistant. A standardized introduction to the tests was given and a standardized glossary of terms was used when the boys asked for explanation 
of a term used in one of the tests. The supervising psychologist had the task to obtain the ICL- $R$ ratings from the group leaders.

\section{Statistical analyses}

The first set of analyses aimed at providing descriptive information concerning the distribution of PCL:YV ratings in the sample. Second, the internal consistency (Cronbach's alpha) and the interrater reliability of PCL: YV total scores, factor scores and individual item scores were examined. Because computation of the inter-item correlation requires a score for each item, the value 1 was assigned to omitted items. Seventeen cases (17\%) for which the PCL:YV coding of only one rater was available, were removed from the analyses concerning interrater reliability. Interrater reliabilities were assessed by means of the intraclass correlation coefficient (ICC). The ICC is an appropriate measure for agreement of variables measured on the same ordinal scale (McGraw \& Wong, 1996). Because there are two sources of variance (random selection of participants from a larger population and random selection of raters from a larger pool of raters), a two way random effects model is used. Consistent with other studies (e.g., Frick, Cornell, Barry, Bodin, \& Dane, 2003; Loney, Taylor, Butler, \& Iacono, 2007; Skeem \& Cauffman, 2003), the absolute agreement type is used to find out if the way of rating by the two raters is identical. ICC's are available as a single measure and an average measure. Average measure ICC's are used when an instrument is coded by multiple raters and the average score will be used as a final score. The single measure ICC is appropriate when the instrument will be rated by only one rater. In this study, both ICC's are reported, so we can examine whether the use of multiple raters increases reliability. ICC's can vary from 0 to 1.0. The following categories are used for evaluating the observed interrater reliability: $\mathrm{ICC} \geq .75=$ excellent; $.60 \leq \mathrm{ICC}<.75=$ good; $.50 \leq$ ICC $<.60=$ moderate ICC $<.50=$ poor (Fleiss, 1986). The reliability of average ratings from two or more raters will be larger than the reliability of only one rater (Shrout \& Fleiss, 1979). 
The relationship between PCL:YV scores and scores on the MMPI-A and ICL-R were examined by computing Pearson product-moment correlation coefficients. Correlations were based on PCL:YV consensus scores or on single ratings when consensus scores were not available. Linear regression with the stepwise procedure (based on the $F$ statistic) was conducted to determine which psychopathy dimension(s) were significant predictors of the personality characteristics measured by the MMPI-A and ICL-R. In addition, linear regression analyses were performed in order to determine whether sexual offending was a moderator of the association between psychopathy scores and the outcome measures. The criterion for entry was set at .05 and for removal at 10 .

\section{RESULTS}

\section{Reliability of individual PCL:YV items}

Table 1 presents descriptive statistics, item-total correlations, and interrater reliabilities for the individual PCL:YV item ratings. PCL:YV item means ranged from .51 (Serious violations of conditional release) to 1.78 (Serious criminal behavior). The single rater ICC's for the individual items ranged from .18 (Lacks goals) to .78 (Criminal versatility; $M d n$ ICC $=.45$; see Table 1). Based on the single ICC, eleven (out of 20) items should be categorized as having poor interrater reliability according to the guidelines provided by Fleiss (1986). The ICC's for the average of two independent raters ranged from .31 (Lack goals) to .88 (Criminal versatility; $M d n$ ICC $=.60$ ). Seven items (of 20) possess poor interrater reliability, based on the average ICC.

All but three items (Lacks goals, Impulsivity and Unstable interpersonal relationships) had corrected item-to-total correlations significant at the .01 level, indicating that most items contribute substantially to the PCL:YV total score (see Table 2). 
TABLE 2

MEAN ITEM SCORES (SD IN PARENTHESES), CORRECTED ITEM-TOTAL CORRELATIONS AND INTERRATER RELIABILITY OF PCL:YV ITEMS (N = 81)

\begin{tabular}{|c|c|c|c|c|c|c|}
\hline & & & & & Reliabilitie & \\
\hline & Item description & Mean & $(S D)$ & Item-total $r$ & ICC single & ICC average \\
\hline 1. & Impression management & .74 & .75 & $.27 * *$ & .27 & .43 \\
\hline 2. & Grandiose sense of self-worth & .90 & .73 & $.55 * *$ & .55 & .71 \\
\hline 3. & Stimulation seeking & 1.11 & .81 & $.60 * *$ & .60 & .75 \\
\hline 4. & Pathological lying & .88 & .76 & $.57 * *$ & .56 & .72 \\
\hline 5. & Manipulation for personal gain & .95 & .73 & $.39 * *$ & .38 & .55 \\
\hline 6. & Lack of remorse & 1.51 & .60 & $.31 * *$ & .32 & .48 \\
\hline 7. & Shallow affect & 1.41 & .54 & $.38 * *$ & .38 & .55 \\
\hline 8. & Callous or lacking empathy & 1.47 & .66 & $.30 * *$ & .31 & .47 \\
\hline 9. & Parasitic orientation & .71 & .73 & $.42 * *$ & .42 & .59 \\
\hline 10. & Poor anger control & 1.38 & .75 & $.65 * *$ & .64 & .78 \\
\hline 11. & Impersonal sexual behavior & 1.30 & .87 & $.70 * *$ & .70 & .82 \\
\hline 12. & Early behavior problems & 1.14 & .84 & $.51 * *$ & .51 & .67 \\
\hline 13 & Lacks goals & 1.07 & .71 & .18 & .18 & .31 \\
\hline 14. & Impulsivity & 1.33 & .71 & $.25 *$ & .25 & .40 \\
\hline 15. & Irresponsibility & 1.34 & .70 & $.38 * *$ & .37 & .54 \\
\hline 16. & Failure to accept responsibility & 1.48 & .66 & $.28 * *$ & .28 & .44 \\
\hline 17. & Unstable interpersonal & & & & & \\
\hline & relationships & 1.08 & .70 & $.22 *$ & .22 & .37 \\
\hline 18. & Serious criminal behavior & 1.76 & .50 & $.57 * *$ & .58 & .73 \\
\hline 19. & Serious violations of & & & & & \\
\hline & conditional release & .51 & .75 & $.64 * *$ & .63 & .78 \\
\hline 20. & Criminal versatility & 1.02 & .83 & $.78 * *$ & .78 & .88 \\
\hline
\end{tabular}

Note. PCL:YV = Psychopathy: Checklist: Youth Version; ICC = intraclass correlation coefficient. $* \mathrm{p}<.05$, two-tailed; $* * \mathrm{p}<.01$, two-tailed. 


\section{Internal reliability of PCL:YV total and factor scores}

The kurtosis of the total PCL:YV consensus score was -.40 $\$ E=.70)$. The scores were normally distributed (Kolmogorov-Smirnov $Z=.55, p=.92$ ). The consensus score ranged from 11 to 39 , with a mean of $24.6(S D=6.2)$, a median of 24 and a mode of 25 . Eighteen boys (22.2\%) could be categorized as low on psychopathic traits (PCL:YV total score $\leq 20), 46$ boys (56.8\%) fell into the moderate range $(20<\mathrm{PCL}$ YY total score $<30)$, and 16 boys $(19.8 \%)$ could be considered high on psychopathic traits (PCL:YV total score $\geq 30$ ).

Table 3 presents internal consistency and interrater reliability of PCL:YV total and factor scores. The single measure ICC for the affective dimension was poor, and good to excellent for the total score, the interpersonal and antisocial dimensions, while moderate for the lifestyle dimension. Average ICC's were excellent for the total score and the interpersonal and antisocial dimensions, good for the lifestyle dimension and moderate for the affective dimension.

Cronbach's coefficient $\alpha$ for the consensus total score indicated good internal reliability (.89). However, the mean inter-item correlation was .17 , which is below the cut-off of .20 for a scale to be considered homogeneous (e.g., Green, Lissitz, \& Mulaik, 1977, Nunnally \& Bernstein, 1994). When the three items with poor item-to-total correlations were excluded from the analysis, Cronbach's $\alpha$ increased to .90 and the mean inter-item correlation increased to an adequate .21 . 
TABLE 3

DESCRIPTIVE STATISTICS AND RELIABILITIES OF PCL:YV TOTAL SCORES $(\mathrm{N}=81)$

\section{PCL:YV}

Reliability index

$\begin{array}{lll}\text { ICC Total } & \text { Single rater } & .74 \\ & & \text { Average rater }\end{array}$

Cronbach's coefficient $\alpha \quad .89$

Mean inter-item $r$

ICC Factor $1 \quad$ Single rater $\quad .64$

Average rater $\quad .78$

Cronbach's coefficient $\alpha \quad .81$

Mean inter-item $r \quad .34$

ICC Factor $2 \quad$ Single rater $\quad .45$

Average rater $\quad .62$

Cronbach's coefficient $\alpha \quad .76$

Mean inter-item $r \quad .29$

ICC Factor $3 \quad$ Single rater $\quad .54$

Average rater $\quad .70$

Cronbach's coefficient $\alpha \quad .73$

Mean inter-item $r \quad .22$

ICC Factor $4 \quad$ Single rater $\quad .68$

Average rater $\quad .81$

Cronbach's coefficient $\alpha \quad .75$

Mean inter-item $r \quad .22$

Note. PCL:YV = Psychopathy: Checklist: Youth Version; ICC = intraclass correlation coefficient. 


\section{Construct validity}

\section{PCL:YV scores and MMPI-A scales}

Table 4 presents an overview of the correlations between PCL:YV total and factor scores and selected MMPI-A scales. In line with our expectations, the PCL:YV total score was significantly positively associated with all MMPI-A scales measuring antisocial/externalizing behaviors (Scale 4, Pd3, conduct problems and anger), except the content scale school problems. Further, psychopathic traits as measured by the PCL:YV were significantly positively related to substance abuse problems (MAC-R, ACK). However, the association between the PCL:YV total score and alcohol/drug problem proneness (PRO) did not reach significance $(r=.20)$. Contrary to our expectations, results indicated no significant associations between PCL:YV total scores and MMPIA scales measuring impulsivity and anxiety. Finally, the PCL:YV total score showed the expected significant positive association with denial of social anxiety (Hy1), and significant negative associations with scales measuring social introversion (Scale 0, Si1, Si2, social discomfort).

The construct validity of the separate psychopathy dimensions was examined by identifying their associations with selected MMPI-A scales. First, in line with our expectations, the interpersonal psychopathy dimension was significantly negatively associated with all scales measuring social introversion. However, the expected significant positive association with denial of social anxiety (Hy1) could not be demonstrated. Since the other psychopathy dimensions also demonstrated significant associations with social introversion, linear regression analysis was performed to identify their unique predictive value. Results demonstrated that while the interpersonal dimension was predictive of a lack of social avoidance $\left(\mathrm{Si} 2 ; R^{2}=.12, p<\right.$ $.01)$ and social discomfort $\left(R^{2}=.07, p<.01\right)$, the lifestyle dimension was predictive of denial of social anxiety ( $\left.\mathrm{Hy} 1 ; R^{2}=.09, p<.01\right)$ and a lack of shyness (Si1; $R^{2}=.11, p<.01$ ). Second, the affective psychopathy dimension did not show the expected significant inverse association with the content scale anxiety. Third, the lifestyle and antisocial dimensions related to all 
MMPI-A scales measuring antisocial/externalizing behavior, except the content scale anger. Findings from linear regression analyses identified the lifestyle dimension as the most significant predictor of authority problems $\left(\mathrm{Pd} 2 ; R^{2}=.14, p<.01\right)$, conduct problems $\left(R^{2}=.13, p<.01\right)$, and school

TABLE 4

CORRELATIONS OF PCL:YV TOTAL AND FACTOR SCORES WITH MMPI-A CLINICAL SCALES, HARRIS-LINGOES SUBSCALES, CONTENT SCALES, AND SUPPLEMENTARY $\operatorname{SCALES}(\mathrm{N}=94)$

PCL:YV

$\begin{array}{lllll}\text { Total } & \text { F1 } & \text { F2 } & \text { F3 } & \text { F4 }\end{array}$

\section{(Antisocial) externalizing behavior}

4 (Psychopathic Deviate)

$\begin{array}{ccccc}.24 * & .04 & .18 & .16 & .31 * * \\ .33 * * & .09 & .20 & .38 * * & .33 * * \\ .25 * & .16 & .19 & .29 * * & .32 * * \\ .27 * & .09 & .17 & .36 * & .10 \\ .14 & -.05 & .12 & .25 * & .04 \\ .25 * & .13 & .22 * & .17 & .13\end{array}$

Pd 2 (Authority problems)

Pd 3 (Social Imperturbability)

Conduct problems

F1

F

F3

School Problems

Anger

Substance abuse problems

Mac-Andrew Alcoholism

$\begin{array}{lllll}.40 * & .28 * & .21 * & .49 * * & .19\end{array}$

Alcohol/Drug Problem

Acknowledgement

$\begin{array}{lllll}.20 & .01 & .11 & .41 * * & .11\end{array}$

Alcohol/Drug Problem Proneness

$.25 *$

.07

.14

$.35 * *$

Note. PCL:YV = Psychopathy Checklist: Youth Version; MMPI = Minnesota Multiphasic

Personality Inventory; F1 = Interpersonal, F2 = Affective, F3 = Lifestyle, F4 = Antisocial; 
problems $\left(R^{2}=.06, p<.05\right)$. The antisocial dimension was the strongest predictor of Scale 4 (Psychopathic Deviate; $R^{2}=.10, p<.01$ ). Notably, the content scale anger was best predicted by the affective psychopathy dimension $\left(R^{2}=.05, p<.05\right)$. The lifestyle dimension was found to be the strongest predictor of alcoholism (MAC-R; $R^{2}=.24, p<.01$ ), alcohol/drug problem acknowledgement (ACK; $R^{2}=.17, p<.01$ ), and alcohol/drug problem proneness $\left(\mathrm{PRO} ; R^{2}=.12, p<.01\right)$. Unexpectedly, the lifestyle dimension was significantly associated with only one of the MMPI-A scales measuring impulsivity (Scale $9 ; r=.21, p<.01 ; R^{2}=.05, p<.05$ ).

Table 4 (continued)

\section{Impulsivity}

Sc 4 (Lack of Ego Mastery-Conative)

.08

$-.05$

.13

.08

$-.01$

Sc5 (Lack of Ego mastery-Defective

Inhibition)

.08

.13

.09

.06

$-.12$

9 (Hypomania)

.15

.09

.12

$.21 * * \quad .01$

Anxiety

.11

.14

.11

.07

$-.03$

\section{Social introversion}

Hy1 (Denial of Social Anxiety)

\begin{tabular}{lllll}
$.24 *$ & .15 & $.20 *$ & $.30 * *$ & $.29 * *$ \\
$-.30 * *$ & $-.28 * *$ & -.14 & $-.34 * *$ & $-.26 *$ \\
$-.32 * *$ & $-.24 *$ & $-.23 *$ & $-.33 * *$ & $-.29 * *$ \\
$-.37 * *$ & $-.35 * *$ & $-.22 *$ & $-.24 *$ & -.19 \\
$-.31 *$ & $-.28 *$ & $-.21 *$ & $-.26 *$ & $-.26 *$ \\
& & & & \\
\hline
\end{tabular}

$* \mathrm{p}<.05$, two-tailed; $* * \mathrm{p}<.01$, two-tailed 


\section{PCL:YV scores and interpersonal styles}

As can be seen in Table 5, PCL:YV psychopathy was related to a self-reported dominant interpersonal style, but, contrary to expectations, not significantly inversely related to self-reported affiliation. Conversely, psychopathic traits were unrelated to mentor-reported dominance, but significantly negatively related to mentor-reported affiliation. Regarding the separate psychopathy dimensions, self-reported dominance was found to be most strongly predicted by the interpersonal psychopathy dimension $\left(R^{2}=.06, p<.05\right)$, whereas the antisocial dimension most strongly predicted mentor-reported lack of affiliation $\left(R^{2}=.05, p<.05\right)$.

TABLE 5

CORRELATIONS OF PCL:YV TOTAL AND FACTOR SCORES, ICL-R SELF RATINGS $(\mathrm{N}=$ 88), AND ICL-R MENTOR RATINGS $(\mathrm{N}=72)$

PCL:YV

\begin{tabular}{lccccc}
\hline & Total & $\mathrm{F} 1$ & $\mathrm{~F} 2$ & $\mathrm{~F} 3$ & $\mathrm{~F} 4$ \\
& & & & & \\
ICL-R self ratings & & & & & \\
Interpersonal style & & & & & \\
$\quad$ Dominance & $.25^{*}$ & $.24^{*}$ & .12 & .21 & $.24^{*}$ \\
$\quad$ Affiliation & -.20 & -.03 & $-.21^{*}$ & -.18 & $-.22^{*}$ \\
ICL-R mentor-ratings & & & & & \\
Interpersonal style & & & & & .10 \\
$\quad$ Dominance & .15 & .18 & .12 & .09 & $-.31^{* *}$ \\
$\quad$ Affiliation & $-.31 * *$ & -.17 & -.21 & $-.25 *$ & \\
\end{tabular}

Note. PCL:YV = Psychopathy Checklist: Youth Version; ICL-R = Interpersonal Checklist-Revised;

$\mathrm{F} 1=$ Interpersonal, $\mathrm{F} 2$ = Affective, F3 = Lifestyle, F4 = Antisocial; * $\mathrm{p}<.05$, two-tailed;

$* * \mathrm{p}<.01$, two-tailed. 
Sexual offenders versus non-sexual offenders

Sexual offending proved to be a significant moderator of the association between PCL:YV total scores and conduct problems $\left(R^{2}=.17, p<\right.$ $.01)$, as well as anxiety $\left(R^{2}=.07, p<.05\right)$. Specifically, correlational analyses demonstrated that in sexual offenders, the PCL:YV total score was significantly associated with conduct problems $(r=.58, p<.01)$. Furthermore, all four dimensions were significantly positively associated with conduct problems (interpersonal: $r=.33, p<.05$; affective: $r=.43, p<$ .01 ; lifestyle: $r=.59, p<.01$; antisocial: $r=.33, p<.05$ ). In contrast, in nonsexual offenders, the PCL:YV total score was unrelated to conduct problems $(r=.06, n s)$, and only the lifestyle dimension demonstrated a significant positive association $(r=.36, p<.05)$. Regarding anxiety, the PCL:YV total score did not show a significant association for either sexual $(r=.26$, $n s)$ or non-sexual $(r=.10, n s)$ offenders. However, in sexual offenders, the interpersonal as well as the lifestyle dimensions demonstrated a modest but significant positive association with anxiety $(r=.32, p<.05$ and $r=.29, p<$ .05 , respectively).

\section{DISCUSSION}

\section{Reliability of the PCL:YV}

This study was the first to evaluate the psychometric qualities of the PCL:YV in a Dutch sample. In this sample of 98 male adolescents admitted to a secure juvenile justice treatment institution, interrater reliabilities of the Dutch language version of the PCL:YV were good to excellent for the PCL:YV total score, good for the interpersonal and antisocial dimensions, moderate for the lifestyle dimension, but poor for the affective dimension. The present results are in line with previous research demonstrating good interrater reliability for the PCL:YV total score (Campbell et al., 2005; Lee et al., 2003; Kosson et al., 2002), and for the traditional two Hare factors (Skeem \& Cauffman, 2003). However, the present reliabilities were less strong than those reported by Campbell et al. (2005). Different procedures for examining interrater 
reliability may account for this. Specifically, for most studies included in the meta-analysis, the PCL:YV was rated by independent professionals, whereas the current study used practicing clinicians. Future research should focus on the extent to which PCL:YV scores are as reliable across examiners in applied settings (particularly in adversial contexts) as they are in research settings (Edens \& Vincent, in press).

Inadequate interrater reliability for the affective dimension of psychopathy, has been previously demonstrated by Spain, Douglas, Poythress and Epstein (2004). They found a highly similar reliability coefficient (single rater ICC $=.43$ ), which they explained by one highly discordant paired rating. In the present study, the four items of the affective factor were among those with the highest means while demonstrating limited variance. In other words, according to the raters of the present study, a deficient affective experience was a general characteristic of the subjects in this sample. In line with the suggestion made by Campbell et al. (2005), it may be difficult for a clinician to judge whether the observed affective deficiency is indeed indicative of psychopathy, in a seriously antisocial sample such as the present one. The present study demonstrated good internal consistency for the PCL:YV total score $(\alpha=.89)$, and dimension scores ( $\alpha$ ranging from $.73-.81$ ). However, according to Nunnally and Bernstein (1994), a reliability of .90 or above is the desirable standard when utilizing scores in a high-stake setting. Although the PCL:YV total score reaches this standard, the present findings indicate dimension scores should not be used in clinical practice. The mean inter-item correlation was below the criterion of .20 (Green et al., 1977; Nunnally \& Bernstein, 1994). This finding was highly similar to that obtained in recent North-American studies of male adolescents on probation (Kosson et al., 2002), and incarcerated male adolescents (Lee et al., 2003; Skeem \& Cauffman, 2003). Further analysis suggested that the relatively low inter-item correlation was due to inadequate item-total correlations for the items Lacks goals, Impulsivity and Unstable interpersonal relationships. These items also demonstrated poor interrater reliability and are among those most criticized (e.g., Hart et al., 2002; Seagrave \& Grisso, 2002), since they may be more characteristic of the general 
adolescent population, rather than specific to psychopathy.

Results from this and other studies suggest that modifications of the PCL:YV may increase reliability of ratings. First, an increase of items defining the separate dimensions may improve the content coverage of the construct to be measured. For example, Frick (2003) recently expanded the callous-unemotional dimension of the Antisocial Process Screening Device (APSD; Frick \& Hare, 2001) to a 24-item rating scale measuring callous and unemotional traits in adolescents; the Inventory of Callous-Unemotional traits (ICU; Frick, 2003). The self-report version of the ICU was found to have good reliability and correlated significantly with measures of conduct problems, psychosocial impairment and the Big Five personality dimensions Agreeableness and Conscientiousness in a community sample of adolescent boys and girls (Essau, Essagawa, \& Frick, 2006). Second, reliability may also be improved by using a multi-method approach (i.e., interviews, observations, psycho(physio)logical assessment, file review) that has been advocated for the assessment of psychopathic traits in pre-adolescent children by Johnstone and Cooke (2004). Finally, more refined item descriptions, indicating when the level of psychopathy symptoms becomes non normative, may increase reliability at the item level (Salekin \& Frick, 2005).

\section{Construct validity}

Psychopathic traits as measured by the PCL:YV total score were associated with a dominant and hostile interpersonal style, antisocial and externalizing behavior and substance abuse problems, and inversely associated with social introversion and a friendly and submissive interpersonal style. The construct validity of the interpersonal dimension was supported by a significant association with a self-reported dominant interpersonal style, and significant inverse associations with MMPI-A scales measuring social introversion. In support of its divergent validity, the interpersonal dimension was demonstrated to be unrelated to antisocial or externalizing behavior, and most MMPI-A scales measuring substance abuse problems. It is somewhat surprising that while adolescents high on psychopathic traits 
acknowledged their dominant interpersonal behavior, the mentors only reported a significant inverse relation to affiliation. One can only speculate about the reason for this discrepancy. It may be that the adolescent with psychopathic traits is not dominant in interpersonal relationships but does endorse these characteristics on a self report instrument because he considers them desirable. Alternatively, the mentor may not realize the adolescent is interpersonally dominant because the adolescent subtly utilizes his dominance to manipulate the mentor. These diverging results increase interest in the quality of the working alliance between the adolescent with psychopathic traits and the mentor.

The affective dimension showed limited associations with external correlates, which is surprising, given that it is generally considered to be the core dimension of psychopathy (Cooke \& Michie, 2001; Vincent, 2002). In an extensive examination of the construct validity of the three Cooke and Michie (2001) factors in an adult sample, Hall et al. (2004) also found the affective factor to have few personality correlates. It was, however, most clearly associated with the more serious forms of antisocial deviance, such as assault, weapons possession and murder (Hall et al., 2004); a finding that has also been demonstrated in previous research in youth (Christian, Frick, Hill, Tyler, \& Frazer, 1997; Frick et al., 2003) and adults (Porter, Woodworth, Earle, Drugge, \& Boer, 2005). Furthermore, psychophysiological responses to emotional stimuli have been successfully used to identify emotional deficits in psychopathic adults (e.g., Patrick, Bradley, \& Lang, 1993), as well as in adolescents with psychopathic traits (Stevens, Charman, \& Blair, 2001). Since psychopaths are characterized by a lack of insight regarding their own emotional deficits, it may be that the affective dimension is relatively resistant to assessment via self-report instruments (Hall et al., 2004). Future research should provide a more comprehensive evaluation of the construct validity of the affective dimension by including psychophysiological variables and observer ratings. Finally, Edens, Skopp and Cahill (2008) found differential effects for the interpersonal and affective dimensions in moderating the association between harsh and inconsistent parental 
discipline and antisocial behavior, suggesting our understanding of the construct validity of the affective dimension may further increase by focusing on its unique effects on associations with external correlates.

Significant debate exists about whether the antisocial dimension should be included in the assessment of psychopathy (Cooke et al., 2004; Hare, 2003; Neumann, Vitacco, Hare, \& Wupperman, 2005). In the present study, the lifestyle and antisocial dimensions generally demonstrated similar patterns of correlations with external criteria. However, there were some discrepancies which may indicate that both dimensions have unique clinical utility as markers of psychopathic traits in youth. Specifically, the lifestyle dimension was demonstrated to be the strongest predictor of impulsivity, authority problems, conduct problems, school problems and substance abuse problems. This is in line with previous findings in adults by Hall and colleagues (2004), leading these authors to suggest that the lifestyle dimension may tap a generalized tendency to engage in socially deviant behaviors, thereby overlapping substantially with the construct of externalizing behavior problems. In contrast, the antisocial dimension was more strongly associated with scale 4 (Psychopathic Deviate) of the MMPI-A, which can be qualified as the most serious indicator of antisocial behavior in this study.

Findings were rather robust across offender types (sexual vs. nonsexual offenders). However, sexual offending was found to be a moderator of the association between psychopathic traits and conduct problems as well as anxiety. Specifically, in the present group of sexual offenders, psychopathic traits were accompanied by conduct problems and anxiety. This finding is in conflict with the generally accepted notion that within the group of childhood-onset conduct-disordered youth, psychopathic traits are only prevalent within a subgroup of youth without anxiety (Frick \& Ellis, 1999; Silverthorn \& Frick, 1999). However, a co-occurrence of psychopathy and clinically significant levels of anxiety or depression has also been demonstrated by Stinson, Becker, and Tromp (2005) in a sample of 68 adult sexual offenders. An explanation of this finding may lie in the widely acknowledged heterogeneity in sexual offenders regarding their risks, 
criminal diversity, treatment needs, and personality profiles (e.g., Boer, Wilson, Gauthier, \& Hart, 1997). Previous findings in adult samples have indicated that (1) psychopathy scores differ across types of sexual offenders (e.g., rapists, child molesters, incest offenders; Barbaree, Seto, Serin, Amos, \& Preston, 1994; Olver \& Wong, 2006) and (2) there is an interaction between psychopathy factor scores and type of sexual offense (Porter et al., 2000). Taking into account the relatively small size of the current sample, more research is needed in order to identify whether the presence of affective disorders is yet another characteristic explaining the heterogeneity in (psychopathic) sexual offenders.

\section{Conclusion}

This study provides initial support for the reliability of the Dutch language version of the PCL:YV for use with male adolescents admitted to a juvenile justice treatment institution. However, interrater reliability findings suggest raters should be cautious when evaluating the affective dimension of psychopathy in adolescents. In addition, researchers should focus on ways to improve reliability, for example by refining the PCL:YV items. Associations between PCL:YV scores and MMPI and ICL-R scales provide support for the construct validity of the total score of the Dutch PCL:YV as well as the interpersonal, lifestyle and antisocial psychopathy dimensions. The construct validity of the affective dimension could not be supported. This may be the result of the poor reliability of this dimension, as well as the limited number of relevant external correlates that were included in the study. More research with different (e.g., noncriminal) and larger samples is needed to further examine the scientific status of the PCL:YV within the Dutch context. This research should also include thorough examination of the convergent and divergent validity by using various methods of assessing the personality dimensions targeted by the PCL:YV. 


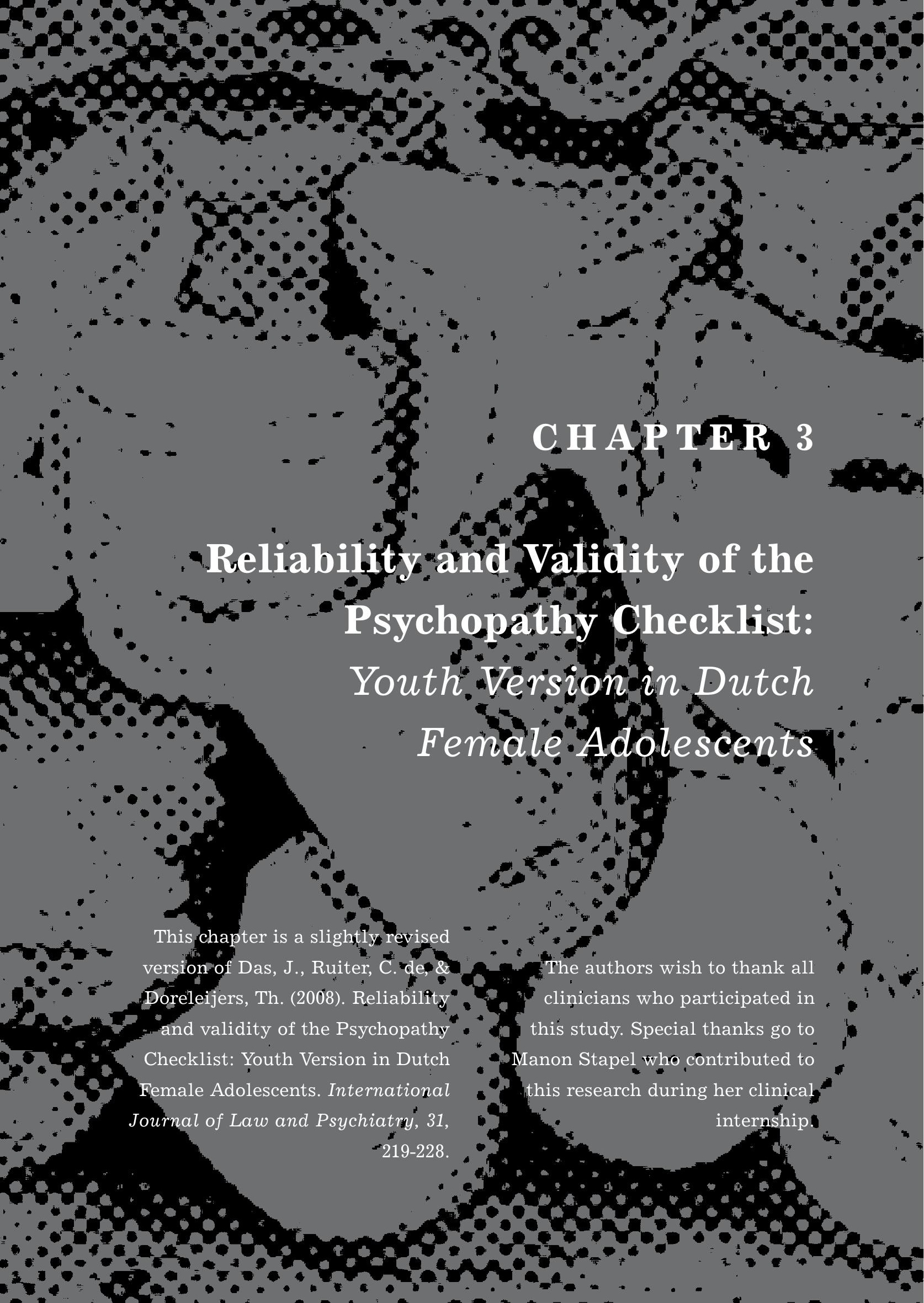




\section{SUMMARY}

In the present study, the reliability and construct validity of the Dutch version of the Psychopathy Check List: Youth Version (Psychopathie Checklist: Jeugd Versie; De Ruiter, Kuin, De Vries \& Das, 2002) were examined in a sample of female adolescents admitted to a secure treatment institution $(\mathrm{N}=67)$. The study provides mixed support for the internal reliability of the PCL:YV in female adolescents. Interrater reliability was found to be adequate at the level of factor and total PCL:YV scores. Poor reliability was demonstrated for the behavioral items of the PCL:YV in particular. Finally, the construct validity of the PCL:YV total and factor scores was supported by theoretically meaningful and significant associations with scores on the Minnesota Multiphasic Personality Inventory (MMPI) and the Interpersonal Checklist-Revised (ICL-R). The applicability of PCL:YV items to female adolescents is discussed.

\section{INTRODUCTION}

An impressive body of evidence has demonstrated that the concept of psychopathy is related to an early onset of antisocial behavior (Forth \& Burke, 1998; Hare, Hart, Forth, Harpur, \& Williamson, 1998), deviant performance on neurocognitive tasks (e.g., Newman \& Schmitt, 1998; Levenston, Patrick, Bradley, \& Lang, 2000), high recidivism rates (Salekin, Rogers, \& Sewell, 1996; Hemphill, Hare, \& Wong, 1998), and a limited response to treatment efforts (Ogloff, Wong, \& Greenwood, 1990; Rice, Harris, \& Cormier, 1992; Seto \& Barbaree, 1999). This has caused researchers to examine the value of extending the psychopathy construct to diverse ethnic and cultural groups (Cooke, Kosson, \& Michie, 2001), females (see for a review, Nicholls, Ogloff, Brink, \& Spidel, 2005), and youths (see for a review, Farrington, 2005). Several scholars have argued that the identification of psychopathic traits in childhood or adolescence may offer a better understanding of the etiology of the disorder and may provide starting points 
for targeting interventions (Forth, Hart, \& Hare, 1990; Forth \& Mailloux, 2000; Frick, Bodin, \& Barry, 2000). For this purpose, the Psychopathy ChecklistRevised (PCL-R; Hare, 1991, 2003), the gold standard for the assessment of psychopathy in adults, has been extended for use with adolescents.

The Psychopathy Checklist: Youth Version (PCL:YV; Forth, Kosson \& Hare, 2003) closely resembles the PCL-R, but item descriptions were modified to take into account the restricted life experience of the adolescent and to emphasize peer contacts instead of romantic relationships (Forth et al., 2003). Similar to the PCL-R, the items of the PCL:YV are scored on a three-point rating scale $(0=$ item does not apply, $1=$ item applies to a certain extent, $2=$ item definitely applies), resulting in a dimensional total score ranging from 0 to 40. In contrast to the PCL-R, there is no designated cut-off score for the PCL:YV. Scores should be interpreted as dimensional ratings of the degree to which an adolescent matches the prototypical psychopath (Forth et al., 2003).

The factor model underlying the PCL:YV is still under investigation. While early studies suggested a similar two factor-structure as the one traditionally thought to underlie adult psychopathy (e.g., Brandt, Kennedy, Patrick, \& Curtin, 1997; Forth \& Mailloux, 2000), the PCL:YV manual (Forth et al., 2003) provides evidence of two alternative models that have been proposed for the PCL-R: a modified version of the hierarchical three-factor model (Cooke \& Michie, 2001) and a parceled four-factor model (Hare, 2003). Research with the PCL:YV in male adolescents has shown some promising predictive validity. For instance, psychopathic traits were found to be associated with violent recidivism (Forth et al., 1990; Gretton, McBride, Hare, O’Shaughnessy \& Kumka, 2001; Gretton, Hare \& Catchpole, 2004), a shorter time span between release and re-offense (Brandt et al., 1997; Gretton et al., 2004), and a higher rate of institutional infractions (Brandt et al., 1997; Skeem \& Cauffman, 2003). 


\section{Psychopathy in females}

The study of psychopathic traits in girls has been stimulated by the fact that antisocial girls are at high risk of adverse long-term outcomes, such as academic difficulties (e.g. school drop out; Bates, Bayles, Bennett, Ridge, \& Brown, 1991), emotional problems (Zoccolillo, 1992), marital difficulties and violent relations with men, (Lewis et al., 1991; Robins, 1986), and poor parenting skills (Lewis et al., 1991). In the PCL:YV manual, it is suggested that the PCL:YV can be applied invariantly across gender (Forth et al., 2003). However, this suggestion may not be warranted considering recent research findings in female adults (e.g., Salekin et al., 1997; Vitale \& Newman, 2001a; Vitale \& Newman, 2001b; Vitale, Smith, Brinkley, \& Newman, 2002). The most striking finding is a lower prevalence rate of psychopathy in a noncriminal female sample (Forth, Brown, Hart, \& Hare, 1996), as well as offender samples (Grann, 2000; Salekin et al., 1997; Salekin, Rogers, Ustad, \& Sewell, 1998; Vitale et al., 2002), than what is typically reported in male forensic samples (Hare, 1991; Hart, Hare, \& Forth, 1994). Furthermore, there is equivocal support for the predictive validity of psychopathy scores in females. For instance, psychopathy was found to be only a poor to modest predictor of future criminal acts in one study (Salekin et al., 1998). Richards, Casey and Lucente (2003), however, identified psychopathy as a better predictor of new criminal charges than a combination of other variables, such as the total number of days spent in treatment and the treatment condition a person was admitted for. In addition, psychopathy scores were associated with poor program adherence, removal for serious noncompliance, violent and disruptive infractions and avoidance of urinalysis testing. These findings are in line with those found for male psychopaths (Ogloff et al., 1990; Rice et al., 1992).

Only a few studies have addressed psychopathy in female adolescents. In their investigation of psychopathic traits as measured by the Antisocial Process Screening Device (APSD; Frick \& Hare, 2001) in a non-referred sample of boys and girls ranging in age from 10 to 17, Marsee, Silverthorn, and Frick (2005) demonstrated that there were no gender differences for 
the callous-unemotional, narcissism and impulsivity dimensions in their associations with aggression and delinquency. However, the psychopathy total score, as well as each psychopathy dimension, was significantly more strongly related to relational aggression in girls than in boys. Contrasting results were found by Odgers, Repucci, and Moretti (2005) in their examination of 125 girls incarcerated at a correctional facility. Using a structural equation modeling procedure, only a moderate relationship between psychopathy scores and concurrent aggression could be identified. More specifically, Factor 2 (Deficient Affective Experience; Cooke \& Michie, 2001) was related to physical ( $=.24, p=.02$ ) and relational $(=.21, p=.01$ ) types of aggression, but this association disappeared when a rivaling variable (experienced victimization) was included in the analysis. Moreover, it was demonstrated that PCL: YV scores were not predictive of future re-offending in girls, whereas prior victimization experiences were.

\section{The present study}

The present study was designed to examine the interrater reliability and internal reliability (item homogeneity and internal consistency) of the Dutch language version of the Psychopathy Checklist: Youth Version (Psychopathie Checklist: Jeugd Versie; PCL:JV; De Ruiter, Kuin, De Vries, \& Das, 2002) in female adolescents. The construct validity of PCL:YV scores will be explored by relating them to scores on the Dutch version of the Minnesota Multiphasic Personality Inventory-Adolescent version (MMPI-A; Butcher et al., 1992; Dutch version: van Dijk, Cornelissen, \& Derksen, 2000) or the MMPI-2 (Butcher, Dahlstrom, Graham, Tellegen, \& Kaemmer, 1989; Dutch version: Derksen, de Mey, Sloore, \& Hellenbosch, 1995) when the subject was 19 years or older. In addition, PCL:YV scores will be related to scores on the Dutch revised version of the Interpersonal Checklist (ICL; LaForge \& Suczek, 1955; Dutch version: ICL-R; De Jong, Van den Brink, \& Jansma, 2000). Previous research in incarcerated male adolescents (Brandt et al., 1997) has demonstrated that PCL:YV scores were positively related to MMPI clinical scales 4 (Psychopathic Deviate) and 9 (Hypomania). Furthermore, 
psychopathy scores have been found to be positively related to a dominant and hostile interpersonal style, and negatively to interpersonal styles characterized by submissiveness and friendliness in male and female students (Forth et al., 1996) and male and female young offenders (Salekin, Leistico, Trobst, Schrum, \& Lochman, 2005). A research question of the present study is whether these findings can be generalized to the present sample of female adolescents in juvenile justice.

\section{METHOD}

\section{Participants}

The sample comprised 67 female adolescents admitted to a secure section of Jongerenhuis Harreveld, a juvenile justice treatment institution. Jongerenhuis Harreveld is a referral institution for the entire nation, which results in the admission of only the most severely disordered girls. The less seriously disturbed girls are taken care of in regional facilities. Most of the girls had been sentenced to a civil supervision order by the court. A supervision order can be imposed when a child's development is psychologically or physically threatened because of incompetent parenting and/or behavioral problems in the child. During the supervision order, the custody of the adolescent becomes the shared responsibility of the parents and the official child protection agency. Girls are also placed in the institution because a mandatory treatment order has been imposed by the juvenile criminal court. The duration of a mandatory treatment order can vary from two to six years, depending on the seriousness of the offense, on whether a mental disorder was present at the time of the offense and on whether the parenting situation was severely compromised.

From January 2002 until June 2003, every newly admitted girl ( $N$ $=81$ ) was asked to participate in the study. Six girls refused to participate and for $89 \%$ of the remaining girls ( $n=67$ ), written informed consent by the adolescent herself and passive informed consent by her parents was obtained. The study was approved by the relevant ethics boards and 
administration of the juvenile justice institution.

\section{Sample characteristics}

The mean age at admission was 15.6 years ( $S D=1.6$; range 11-19). In terms of ethnic origin, $72 \%$ was European, $6 \%$ was Surinamese, $3 \%$ was Moroccan, $2 \%$ was from the Netherlands Antilles, and $18 \%$ had another ethnic background (i.e., Asian, South-American, Turkish, North-African or Russian). Furthermore, $93 \%$ of the girls were placed in the institution under a supervision order and $8 \%$ under a mandatory treatment order. All four girls with a mandatory treatment order were convicted for a violent offense: manslaughter $(n=1)$, murder $(n=2)$ and violent robbery $(n=1)$. Although girls with a supervision order were not convicted for a criminal offense, they showed serious externalizing behavioral problems, including running away from home, substance abuse and engaging in prostitution, as well as internalizing behavior problems. At the time the present study was finished, eight girls were still in the institution. The mean length of stay of the other 59 girls was 16.08 months ( $\mathrm{SD}=8.2$ ). The girls who did not participate in the study did not differ from girls in the present sample in terms of age ( $t$ $=-1.00, p=.32)$, ethnicity $\left(\chi^{2}(4)=2.99, \mathrm{p}=.60\right)$ and the judicial measure that was imposed by the court $\left(\chi^{2}(2)=.66, \mathrm{p}=.72\right)$. Finally, the girls who participated in the study differed somewhat from the total population of girls who were admitted to a juvenile justice treatment institution in The Netherlands between 1995 and 2005. Specifically, the mean age of the total population $(N=789)$ was somewhat lower $(14.82, S D=1.24)$. Furthermore, fewer girls from the overall population (1.4\%) were admitted under a mandatory treatment order and more (98.6\%) under a supervision order. However, the ethnic make-up of the Harreveld sample was highly similar to that of the general population of girls in juvenile justice institutions: $75.4 \%$ European, 7.1\% Surinamese, 3.2\% Moroccan, 3.0\% Netherlands Antilles, and $11.3 \%$ had another ethnic background. 


\section{Instruments}

$P C L: Y V$

PCL:YV ratings were based on the administration of the Dutch translation (Vertommen, Verheul, De Ruiter and Hildebrand, 2002) of the semistructured PCL-R interview designed by Hare (1991) together with collateral information available for each adolescent upon admission, including police files, psychiatric and psychological evaluations, and observational reports from previous institutional care. Each subject was rated by two independent raters. For some PCL: YV items, the descriptive criteria were slightly revised from the original Canadian version, because during the consensus meetings it became apparent that some of the scoring criteria were unclear. A clarification was added to the item description in a separate textbox (see Table 1 for an overview of these revisions). The PCL:YV consensus score for all but six girls (9\%) was based on the revised criteria. For these six girls, the items were recoded according to the revised criteria.

The traditional two-factor model was used, wherein Factor 1 is characterized by a selfish and callous interpersonal style and a lack of remorse and empathy, and Factor 2 by a chronically unstable and antisocial lifestyle (Harpur, Hakstian, \& Hare, 1988).

\section{$M M P I-A$ and MMPI-2}

In this study the MMPI-A (Butcher et al., 1992; authorized Dutch version: Van Dijk et al., 2000) was used. Norms are available for adolescents between 13 and 19 years of age. The MMPI-A is a 478-item self-report questionnaire which offers insight into psychopathology and personality traits. The instrument consists of a number of validity scales, which allow insight into the response style of the adolescent (e.g., socially desirable or inconsistent responding). Furthermore, nine clinical scales, 15 content scales and six supplementary scales provide information on the presence or absence of a large variety of psychopathological symptoms. Research has demonstrated that American adolescents score significantly higher on the MMPI-A than 
TABLE 1

REVISIONS OF ITEM DESCRIPTIONS

PCL:YV-item

Item 9: Parasitic orientation

Item 11: Impersonal sexual behavior

Item 13: Lacks goals

Item 17: Unstable interpersonal relationships

Item 18: Serious criminal behavior
A score of 1 can be given in case the adolescent uses criminal activity to provide financially for himself or does not have any clear means of financial support.

For scoring this item, emphasis is placed on infidelity, voluntary prostitution, extremely high frequency of impersonal sexual relationships ('players') and / or a great variety of sexual activity.

Takes into account whether the adolescent has demonstrated to be committed to short term goals.

A score of 1 can be given when there is no clear sign of instability of the relationships, but the relationships can be characterized as quite superficial.

The item description was replaced by the item description of this item in the PCL-R

Dutch adolescents, suggesting Dutch norms should be used (Derksen, Van Dijk, \& Cornelissen, 2003). Furthermore, the test-retest reliability, based on a one-week interval, of the Dutch MMPI-A was found to be reasonable and the internal consistency of most scales was adequate, except for two clinical scales [Scale 3 (Hysteria) and Scale 5 (Masculinity/femininity)], one content scale (low ambition) and one supplementary scale (MacAndrew Alcoholism). Finally, findings supported the construct validity of the Dutch MMPI-A (Derksen et al., 2003). When a subject was 19 years or older, the MMPI-2 (Butcher et al., 1989; authorized Dutch edition: Derksen et al., 1995) was used (this was the case for $5 \%$ of the girls who agreed to complete the MMPI).

In this study, MMPI-A profiles were considered invalid when scores on the TRIN (True Response Inconsistency) and/or VRIN (Variable Response 
Inconsistency) scales were over 75, and MMPI-2 scores were considered invalid when VRIN scores were over 80. Eighty-eight percent of the girls agreed to complete the MMPI, with $80 \%$ of the girls $(n=54)$ having a valid profile. Mean PCL:YV scores for girls with a valid MMPI profile did not differ from girls who either did not fill out the MMPI or had an invalid profile $(t=-1.54$, $p=.25)$.

ICL-R

The authorized Dutch translation of the Interpersonal CheckList-Revised (ICL-R; de Jong et al., 2000) was used to map interpersonal behavioral styles. The ICL-R is an updated version of the Interpersonal CheckList (LaForge \& Suczek, 1955) and is based on Leary's model of interpersonal behavior (1957). The ICL-R consists of 160 items, which can be rated yes or no. The instrument consists of ten behavioral dimensions: PA (managerial-autocratic), BC (competitive-exploitative), DE (aggressive-blunt), FG (distrustful-skeptical), nFnG (reserved-aloof), HI (modest-self-efficacing), JK (docile-dependent), LM (cooperative-overconventional), NO (responsible-overgenerous), and $\mathrm{nNnO}$ (extravert-gregarious). The scores on the ten dimensions can be transformed into a vector score, describing whether the interactional style is predominantly characterized by dominance versus submissiveness, and by hostility versus friendliness. The ICL-R manual provides information on the psychometric properties of the ICL-R in Dutch samples. The intercorrelations and the circumplex analysis support the hypothesized circular arrangement of the interpersonal styles. The test-retest reliability of the interpersonal styles is moderate to good (ICC range = .57 - .83; de Jong et al., 2000).

ICL-R ratings were obtained from the girls themselves as well as from group leaders (observer ratings) who interact with the girls on a daily basis. Both ratings were used separately in the analyses. The response rates were $87 \%$ and $85 \%$, for the group leaders and the girls, respectively.

\section{Procedure}

All raters $(N=12)$ received training in the administration and scoring of the 
PCL-R by the second author (Corine de Ruiter, Ph.D.) and/or Pascalle van der Wolf, M.Sc., who both had been trained by Drs. Robert D. Hare and David Cooke. The training included a review of the clinical construct of psychopathy and the research literature pertaining to it. Scoring was practiced using videotapes of two Dutch adult forensic psychiatric patients and three adolescent offenders.

In most cases, the participant was interviewed and rated by the supervising psychologist of the ward. This procedure was chosen for several reasons. In the first place, it allowed the psychologist to get to know the client by interviewing her, scoring the PCL:YV and the psychological tests. Secondly, the interview was the first occasion for extended contact and provided a possible basis for a (good) working alliance with the adolescent. Finally, we believe it is important to investigate whether the PCL:YV can be reliably coded by practicing clinicians since this partially determines its ecological validity. The PCL-R interviews were videotaped and after the independent coding of the PCL:YV by two raters, a meeting was planned to obtain a final (consensus) rating for the adolescent. This procedure was chosen in order to maximize information exchange and facilitate precision in scoring.

The administration of the MMPI-A/MMPI-2 and the ICL-R took place in groups of three adolescents at a time, supervised by a psychologist or a research assistant. A standardized introduction to the tests was given and a glossary of difficult terms was used when the girls asked for an explanation of a term used in one of the tests. The supervising psychologist of the ward made sure ICL-R ratings were obtained from the group leaders.

\section{Statistical analyses}

The first set of analyses aimed at providing descriptive information concerning the distribution of PCL:YV ratings in the sample. Second, internal consistency (Cronbach's alpha) and interrater reliability of PCL: YV total scores, factor scores and individual item scores were examined. Because computation of the inter-item correlation requires a score for each 
item, the value 1 was assigned to omitted items. Interrater reliability was assessed by means of the intraclass correlation coefficient (ICC). The ICC is an appropriate measure for agreement of variables measured on the same ordinal scale (McGraw \& Wong, 1996). Because there were two sources of variance (random selection of participants from a larger population and random selection of raters from a larger pool of raters), a two way random effects model was used. The consistency type was used to find out whether the manner of rating by the two raters was comparable (and not identical, because in that case the absolute agreement type would be used). A single measure ICC and an average measure ICC can be distinguished. Average measure ICCs are used when an instrument is coded by multiple raters and the average score will be used as a final score. The single measure ICC is the parameter of choice when the instrument is rated by only one rater. In this study, both ICCs are reported, so it can be established whether the use of multiple raters increases reliability. ICCs can vary from 0 to 1.0. The following categories were used for evaluating the observed interrater reliability: $\mathrm{ICC} \geq .75=$ excellent; $.60 \leq \mathrm{ICC}<.75=$ good; $.50 \leq \mathrm{ICC}<.60$ $=$ moderate ICC $<.50=$ poor (Fleiss, 1986). Three cases for which the PCL: YV coding of only one rater was available, were removed from the analyses concerning interrater reliability.

Finally, the relationship between the scores on the PCL:YV, the MMPIA, the MMPI-2 and the ICL-R was examined by computing Pearson productmoment correlation coefficients.

\section{RESULTS}

\section{Individual PCL:YV items}

Table 2 presents descriptive statistics, item-total correlations and interrater reliabilities of the individual PCL:YV item scores. PCL:YV mean item scores ranged from 0.46 (serious violations of conditional release) to 1.77 (irresponsibility). The ICCs for the individual items ranged from -.01 (serious violations of conditional release) to .62 (serious criminal behavior) for a 
single rater $(M d n=.39:$ see Table 2 ). Sixteen (out of 20 ) items could be categorized as having poor interrater reliability according to the guidelines provided by Fleiss (1986). The ICCs for the average of two independent raters ranged from -.02 (serious violations of conditional release) to .76 (serious criminal behavior; $M d n \mathrm{ICC}=.56$ ). Based on the average $\mathrm{ICC}$, only six items (of 20) could be categorized as having poor interrater reliability, suggesting improved reliability when the PCL:YV is coded by two raters instead of one.

All but five items (impersonal sexual behavior, early behavior problems, lacks goals, impulsivity and serious violations of conditional release) revealed corrected item-to-total correlations $\geq .35$, indicating that most items contributed significantly to the PCL:YV total score (see Table 2). Items related to irresponsible behavior had a less strong association with the total score.

\section{PCL:YV total and factor scores}

The kurtosis of the consensus score was .02 $\$ E=.59)$. The scores were normally distributed (Kolmogorov-Smirnov $Z=.98, p=.30$ ). The consensus score ranged from 7 to 35 with a mean of $23.72(S D=6.13)$, a median of 25 and a mode of 25 . Table 3 presents the internal consistency and interrater reliability of PCL:YV total and factor scores. The single ICCs for the total and factor scores of the PCL:YV could be categorized as moderate to good (ICC for Factor $1=.60$ ), whereas average ICCs were good to excellent.

Cronbach's coefficient $\alpha$ for the consensus total score indicated high internal reliability (.79). However, the mean inter-item correlation was .16 , which is below the suggested cut-off of .20 in order for a scale to be considered homogeneous (Green, Lissitz, \& Mulaik, 1977).

When the five items with poor item-to-total correlations (Impersonal sexual behavior, Early behavior problems, Lacks goals, Impulsivity and Serious violations of conditional release) were excluded from the analyses, Cronbach's $\alpha$ increased to .83 and the mean inter-item correlation increased to an adequate .23 . 
TABLE 2

MEAN ITEM SCORES, STANDARD DEVIATIONS, CORRECTED ITEM-TOTAL

CORRELATIONS AND INTERRATER RELIABILITY OF PCL:YV ITEMS $(N=64)$

Reliabilities

Item description

Mean

(SD)

Item-total $r$

ICC single

ICC average

rater

measure

1. Impression management

$.95 \quad .76$

.39

.37

.54

2. Grandiose sense of self-worth

$.83 \quad .72$

.39

.33

.50

$3 \quad$ Stimulation seeking

$1.70 \quad .49$

.39

.41

.58

4. Pathological lying

$.95 \quad .65$

.58

.41

.59

5. Manipulation for personal gain

$1.28 \quad .67$

.49

.33

.49

6. Lack of remorse

$1.54 \quad .66$

.66

.31

.47

7. Shallow affect

$\begin{array}{ll}1.32 & .75\end{array}$

.59

.43

.60

8. Callous or lacking empathy

$1.34 \quad .74$

.66

.49

.66

9. Parasitic orientation

$\begin{array}{ll}.83 & .75\end{array}$

.35

.34

.51

10. Poor anger control

$1.39 \quad .68$

.44

.37

.54

11. Impersonal sexual behavior

$1.00 \quad .89$

.29

.54

.70

12. Early behavior problems

$.78 \quad .86$

.31

.53

.69

13. Lacks goals

$1.02 \quad .76$

.34

.45

.62

14. Impulsivity

$1.66 \quad .54$

.26

.05

.10

15. Irresponsibility

$1.77 \quad .46$

.41

.30

.46

16. Failure to accept responsibility

$1.63 \quad .58$

.70

.46

.63

17. Unstable interpersonal

relationships

$1.46 \quad .59$

.48

.08

.15

18. Serious criminal behavior

$1.17 \quad .74$

.55

.62

.76

19. Serious violations of conditional

release

$.46 \quad .66$

.11

$-.01$

$-.02$

20. Criminal versatility

$.63 \quad .76$

.55

.51

.68

Note. $\mathrm{PCL}: \mathrm{YV}=$ Psychopathy: Checklist: Youth Version; ICC = intraclass correlation coefficient. 
TABLE 3

RELIABILITIES OF PCL:YV SCORES $(\mathrm{N}=64)$

PCL:YV

Reliability index

\begin{tabular}{|c|c|c|}
\hline Total & Single rater & .57 \\
\hline & Average rater & .72 \\
\hline Factor 1 & Single rater & .60 \\
\hline & Average rater & .75 \\
\hline Factor 2 & Single rater & .51 \\
\hline & Average rater & .68 \\
\hline Cronbach's coef & ient $\alpha$ & .79 \\
\hline Mean inter-item & & .16 \\
\hline
\end{tabular}

Note. PCL:YV = Psychopathy: Checklist: Youth Version; SD = Standard deviation; Mdn = Median; ICC = intraclass correlation coefficient.

\section{Construct validity}

In Table 4 an overview is presented of the correlations between PCL:YV total and factor scores and MMPI clinical scales and subscales. Psychopathic traits in girls as measured by the consensus score on the PCL:YV, appeared to be strongly related to scale $5(\mathrm{Mf} ; r=.41)$. Scale 5 is characteristic of women who "... tend to be assertive, competitive, tough-minded and not particularly interested in appearing or behaving as other women do. Instead, they are more likely to be independent, self-confident, spontaneous, dominant and even aggressive in thought and action." (Friedman, Lewak, Nichols \& Webb, 2001, p. 317). Furthermore, an absence of somatic complaints (scale 3; Hypochondriasis; scale Hy4; somatic complaints) and an absence of 
sensitivity in reaction to others (scale $\mathrm{Pa} 2$ poignancy) characterized these girls. Finally, inhibition of aggression (Hy5) and social avoidance (Si2) were negatively related to PCL:YV total consensus scores.

With respect to Hare's traditional Factor 1, a significant positive association with scale 5 (Mf; $r=.38$ ) and significant negative associations with somatic complaints (scale 3 ; Hy), sensitivity in reaction to others ( $\mathrm{Pa} 2$ poignancy) and social avoidance (Si2) were demonstrated. Furthermore, significant relations with other specific MMPI scales could be identified. First, the results revealed that the interpersonal and affective domains of psychopathy were related to social imperturbability (Pd3; Ma3), suggesting that these girls have little concern about the opinions, values and attitudes of others. Second, girls with a high Factor 1 score were characterized by an absence of bodily complaints (scale 1; Hypochondriasis) and an absence of feelings of weakness, tiredness and loss of interest in life (Hy3). Finally, high PCL:YV Factor 1 scores were inversely related to the disruption of information processing by the intrusion of troubling thoughts (Sc3).

Associations between Hare's traditional Factor 2 and MMPI scales could also be identified. For instance, Factor 2 was significantly positively related to authority conflict ( $\mathrm{Pd} 2)$ and significantly negatively related to psychomotor retardation (D2), somatic complaints (Hy4), inhibition of aggression (Hy5) and social avoidance $(\mathrm{Si} 2)$.

The results concerning the relationship between PCL:YV scores and MMPI content and supplementary scales are presented in Table 5. Factor 1 scores were inversely related to health concerns, low self-esteem and anxiety. Furthermore, girls high on Factor 2 demonstrated an elevation on the anger (ANG) and conduct problems scales (A-con), while showing proneness to alcohol or drug problems (PRO) and psychological immaturity (IMM). 
TABLE 4

CORRELATIONS OF PCL:YV TOTAL AND FACTOR SCORES WITH MMPI CLINICAL

SCALES AND HARRIS-LINGOES SUBSCALES $(\mathrm{N}=54)$

PCL:YV

MMPI-A or MMPI-2 clinical-

Total score

Factor 1 score

Factor 2 score

or subscale

1 (Hypochondriasis)

D (Depression)

D1 (Subjective Depression)

D2 (Psychomotor Retardation)

D3 (Physical Malfunctioning)

D4 (Mental Dullness)

D5 (Brooding)

3 (Hysteria)

Hy1 (Denial of Social Anxiety)

Hy2 (Need for affection)

Hy 3 (Lassitude-Malaise)

Hy 4 (Somatic Complaints)

Hy 5 (Inhibition of Aggression)

4 (Psychopathic Deviate)

Pd1 (Familial Discord)

Pd 2 (Authority problems)

Pd 3 (Social Imperturbability)

Pd 4 (Social Alienation)

Pd 5 (Self Alienation)

5 (Masculinity-Femininity)

6 (Paranoia)

Pa1 (Ideas of External Influence)

$\mathrm{Pa} 2$ (Poignancy)
$-.21$

$-.11$

$-.05$

$-.27$

$-.23$

$-.03$

$-.10$

-.27 *

.18

$-.06$

$-.17$

-.28 *

-.28 *

.13

$-.00$

.23

.22

$-.02$

.04

$.41 * *$

$-.13$

$-.09$

$-.32 *$
$-.33 *$

$-.22$

$-.21$

$-.21$

$-.27$

$-.20$

$-.25$

$-.31 *$

.25

.09

-.30 *

$-.05$

$-.05$

$-.03$

$-.07$

.06

$.31 *$

$-.14$

$-.14$

.38

$-.25$

$-.18$

$-.37$
$-.06$

$-.07$

.06

$-.29 *$

$-.11$

.08

.05

$-.18$

.09

$-.15$

$-.09$

$-.39 * *$

$-.39 * *$

.22

.02

.33 **

.11

.10

.13

.27

.03

.06

$-.14$ 
Pa3 (Moral Virtue)

7 (Psychasthenia)

8 (Schizophrenia)

Sc1 (Social Alienation)

Sc 2 (Emotional Alienation)

Sc 3 (Lack of Ego Mastery-Cognitive)

Sc 4 (Lack of Ego Mastery-Conative)

Sc5 (Lack of Ego mastery-Defective

Inhibition)

Sc6 (Sensorimotor Dissociation)

9 (Hypomania)

Ma1 (Amorality)

Ma2 (Psychomotor Acceleration)

Ma3 (Imperturbability)

Ma4 (Ego Inflation)

0 (Social Introversion)

Si1 (Shyness)

Si2 (Social Avoidance)

$\mathrm{Si} 3$ (Self / Other alienation)
$-.01$

$-.11$

$-.13$

$-.14$

$-.13$

$-.17$

$-.07$

$-.10$

$-.11$

.00

$-.07$

$-.11$

$-.17$

$.31 *$

.02

$-.22$

$-.14$

-.30 *

$-.21$

$-.08$
$-.13$

$-.00$

.01

$-.00$

$-.01$

$-.02$

.05

$-.01$

$-.05$

.12

.12

.11

.17

.01

$-.10$

$-.06$

-.32 *

.06

Note. PCL:YV = Psychopathy Checklist: Youth Version; MMPI = Minnesota Multiphasic

Personality Inventory; $* \mathrm{p}<.05$, two-tailed, $* * \mathrm{p}<.01$ level, two-tailed. 
TABLE 5

CORRELATIONS OF PCL:YV TOTAL AND FACTOR SCORES AND MMPI CONTENT AND

SUPPLEMENTARY SCALES $(\mathrm{N}=54)$

PCL:YV

MMPI-A or MMPI-2 content scales

Total score

Factor 1 score

Factor 2 score

Anxiety

$-.10$

$-.24$

$-.03$

Obsessiveness

$-.08$

$-.18$

$-.00$

Depression

$-.11$

$-.25$

.01

Health Concerns

$-.22$

$-.33 *$

$-.05$

Alienation

$-.13$

$-.19$

$-.14$

Bizarre Mentation

$-.15$

$-.23$

$-.04$

Anger

.13

$-.08$

.33 *

Cynicism

$-.08$

$-.15$

$-.01$

Conduct problems

.18

.01

$.31 *$

Low self esteem

$-.20$

$-.36 * *$

$-.04$

Low Ambition

.08

$-.06$

.17

Social Discomfort

$-.20$

$-.24$

$-.16$

Family Problems

$-.01$

$-.07$

.01

School Problems

.05

$-.18$

.24

Negative Treatment Indicators

$-.07$

$-.25$

Supplementary Scales

Anxiety

$-.17$

$-.28 *$

$-.04$

Repression

$-.12$

$-.01$

$-.24$

Mac-Andrew Alcoholism

.16

$-.04$

.27

Alcohol/Drug Problem Acknowledgement

.13

$-.13$

.26

Alcohol/Drug Problem Proneness

.26

.01

Immaturity

.08

$-.14$

Note. PCL:YV = Psychopathy Checklist: Youth Version; MMPI = Minnesota Multiphasic

Personality Inventory; $*<<.05$, two-tailed, $* * p<.01$ level, two-tailed. 
Tables 6 displays the results pertaining to the ICL-R self and group leader ratings in relation to the $\mathrm{PCL}: \mathrm{YV}$. A significant negative association was found between the PCL:YV Factor 1 score and a self-reported reservedaloof interpersonal style, whereas a positive association was demonstrated with the dominance vector. Furthermore, the PCL:YV Factor 1 score was significantly associated with a group leader-rated competitive-exploitative and overall dominant interpersonal style, while PCL:YV total scores were negatively related to a cooperative-overconventional and overall friendly interpersonal style. Finally, Factor 2 scores were associated with a distrustful and skeptical interpersonal style, and inversely related to a cooperativeoverconventional and friendly interpersonal style as rated by the group leaders.

TABLE 6

CORRELATIONS OF PCL:YV TOTAL AND FACTOR SCORES, ICL-R SELF RATINGS $(N=$ 55), AND ICL-R OBSERVER RATINGS ( $=57)$

\begin{tabular}{|c|c|c|c|c|c|c|}
\hline \multirow[b]{2}{*}{ ICL-R } & \multicolumn{3}{|c|}{$\begin{array}{l}\text { ICL-R self } \\
\text { PCL:YV }\end{array}$} & \multicolumn{3}{|c|}{$\begin{array}{c}\text { ICL-R mentor } \\
\text { PCL:YV }\end{array}$} \\
\hline & Total & Factor 1 & Factor 2 & Total & Factor 1 & Factor 2 \\
\hline Interpersonal style & score & score & score & score & score & score \\
\hline PA (managerial-autocratic) & .11 & .17 & -.04 & .03 & .20 & -.09 \\
\hline BC (competitive-exploitative) & .21 & $.30 *$ & .05 & .04 & .22 & -.11 \\
\hline DE (aggressive-blunt) & .25 & .21 & .16 & .22 & .04 & .19 \\
\hline FG (distrustful-skeptical) & .21 & .08 & $.29 *$ & .05 & -.10 & .13 \\
\hline nFnG (reserved-aloof) & -.09 & -.12 & .07 & -.23 & $-.28 *$ & -.10 \\
\hline HI (modest-self-efficacing) & -.17 & $-.36 * *$ & -.09 & -.18 & -.21 & -.07 \\
\hline JK (docile-dependent) & -.19 & -.20 & -.08 & -.03 & -.11 & .08 \\
\hline
\end{tabular}




\begin{tabular}{lcccccc}
\hline & \multicolumn{3}{c}{ ICL-R self } & \multicolumn{3}{c}{ ICL-R mentor } \\
Table 6 (continued) & \multicolumn{3}{c}{ PCL:YV } & & \multicolumn{2}{c}{ PCL:YV } \\
& & & & & \\
LM (cooperative- & & & & & \\
overconventional) & $-.27 *$ & -.08 & $-.33 *$ & .01 & .12 & -.04 \\
NO (responsible-overgenerous) & -.16 & -.04 & -.25 & -.02 & .05 & -.06 \\
nNnO (extravert-gregarious) & -.03 & .12 & -.14 & .21 & .27 & .21 \\
Dominance & .12 & $.32 *$ & -.14 & .15 & $.29 *$ & .00 \\
Affiliation & $-.28 *$ & -.14 & $-.30 *$ & -.01 & .06 & -.05 \\
& & & & & & \\
\hline
\end{tabular}

Note. PCL:YV = Psychopathy Checklist: Youth Version; ICL-R = Interpersonal Checklist-Revised; $* \mathrm{p}<.05$, two-tailed, $* * \mathrm{p}<.01$ level, two-tailed.

\section{DISCUSSION}

\section{Reliability}

Mixed support was found for the reliability of the PCL:YV in female adolescents. With regard to internal consistency, Cronbach's $\alpha(.79)$ was found to be excellent, but the mean inter-item correlation was relatively low (.16). Furthermore, item-total correlations were demonstrated to be adequate ( $>$ .35) for all but five items. The items Impersonal sexual behavior, Early behavior problems, Lacks goals, Impulsivity and Serious violations of conditional release did not contribute significantly to the PCL:YV total score. Excluding these items from the analyses led to an improved Cronbach's $\alpha$ and the mean inter-item correlation then exceeded the suggested criterion of .20 (Green et al., 1977), indicating adequate homogeneity of the PCL:YV. Single rater ICCs indicated moderate to good interrater reliability of the PCL:YV total and factor scores. The reliability improved considerably when the PCL: $\mathrm{YV}$ was coded by two independent raters, a finding which is in line with previous research (Vitale et al., 2002). A poor interrater reliability was found for most of the individual items. 
Several explanations may be offered for the failure to provide convincing evidence for the reliability of the PCL:YV in our female adolescent sample. First, the age-appropriateness of some of the PCL:YV item descriptions has been called into question because of the downward extension of PCL-R items that was used in the development of the PCL:YV (Hart, Watt, \& Vincent, 2002; Seagrave \& Grisso, 2002). The PCL:YV manual seems to lack clear instructions as to what should be considered psychopathic trait-like and what should be seen as transient, state-like characteristics that are a normal part of adolescence. In addition, the restricted life experience of adolescents complicates the coding of some of the items. When there is evidence of a pattern of behavior, one can be more sure that certain behaviors are trait-like (which is often the case with adults, who have just had a longer lifetime to show the behaviors) than when the time frame is short (as is the case with adolescents). The poor interrater reliability at the item level may be partly the result of this indistinctness of PCL:YV item descriptions.

Second, the improved internal consistency after the exclusion of five behaviorally oriented items suggests that these PCL:YV items may not be appropriately defined for diagnosing psychopathic traits in girls. Several authors suggested that the DSM-IV diagnostic criteria for Conduct Disorder (CD) appear to be more relevant to boys because they emphasize overt aggressive and antisocial behavior, while more covert types of antisocial behavior, such as sexual promiscuity, prostitution, frequent lying and manipulativeness are more characteristic of girls (Pepler, Madsen, Webster, \& Levene, 2005; Odgers \& Moretti, 2002). Similarly, there may be gender bias in the diagnosis of psychopathy (Cale \& Lilienfeld, 2002). Indeed, Salekin and Schrum (2006) used Item Response Theory (IRT) to analyze the applicability of the PCL:YV to a sample of 123 detained girls. Some items were found to be less applicable to girls, among these were Poor anger control, Serious violations of conditional release, and Parasitic lifestyle. Furthermore, some items that have not been found to be good disciminators in adult samples 


\section{Validity}

Theoretically significant associations between the PCL:YV, MMPI and ICL-R provided support for the construct validity of the PCL:YV total and factor scores. According to Fiske and Campbell (1992), validity coefficients regarding construct validity are often modest, typically in the .30 to .50 range. By these standards, a strong association was found between psychopathy scores and MMPI-scale 5 (Mf), which characterized these girls as assertive, competitive, tough-minded, self-confident, dominant and aggressive. Furthermore, the absence of physical complaints, social avoidance, low self-esteem and anxiety, and the presence of behavioral problems (i.e., anger proneness, problems related to alcohol and drug use, disinhibition of aggression) and little concern about the values of others were characteristic of these girls. Factor 1 was mainly related to the absence of social avoidance, somatic complaints and feelings of lethargy, while Factor 2 was related to behavioral problems like authority conflict and disinhibition of aggression. We could not replicate the significant associations between psychopathy scores and scale 4 and a combination of scales 4 and 9 of the MMPI, demonstrated previously in a sample of male adolescent offenders (Brandt et al., 1997).

The associations between the PCL:YV and the ICL-R self-report and observer ratings demonstrated that girls scoring high on the PCL:YV had a dominant and hostile interpersonal style. Factor 1 was especially associated with interpersonal dominance. These results are in line with findings from a study conducted by Forth et al. (1996), who demonstrated in a noncriminal adult female sample, that psychopathy scores were associated with a selfreported and observed dominant, arrogant, calculating and cold-hearted interpersonal style. In summary, our study provides initial evidence for the concurrent and divergent validity of the Dutch PCL:YV in female adolescents.

\section{Limitations}

A first limitation of our study concerns the relatively small sample size of 67 girls. A larger sample might have increased the reliability estimates. 
However, given the paucity of research on psychopathic traits in female adolescents, even a study with a sample of limited size such as the present one, can make a contribution to the knowledge base.

Secondly, in the present study PCL:YV consensus ratings were used in the analyses concerning construct validity, which is in contrast to previous studies, which used psychopathy scores obtained from a single rater. We believe a consensus rating may be advisable for obtaining a reliable psychopathy score in female adolescents, because of the exchange of information and the integration of this information during the consensus meeting, an approach that has also been advocated by Cooke et al. (2004).

Thirdly, the sample was derived from a single Dutch juvenile justice treatment institution, thereby limiting generalization. Nevertheless, a comparison with demographic characteristics of the total population of girls admitted to a secure institution, indicates that our group is largely representative.

\section{Clinical implications}

The present findings regarding reliability suggest that PCL:YV item descriptions may have to be modified to take into account gender-specific manifestations of psychopathy. In the meantime, clinicians should be reticent about using the PCL:YV with female adolescents in clinical practice. Only factor and total scores should be used, whereas item scores are not reliable. Furthermore, a consensus meeting between independent raters is recommended to increase the reliability of PCL:YV scores in girls. Despite the present shortcomings in reliability, our findings do provide initial evidence of the construct validity of the PCL:YV for measuring psychopathic traits in girls. Although much is still unknown about the possible long term consequences of these traits in girls (e.g., risk of antisocial personality disorder, treatment responsiveness), taking into account their psychopathic characteristics may prove an advancement in the delivery of treatment interventions. 


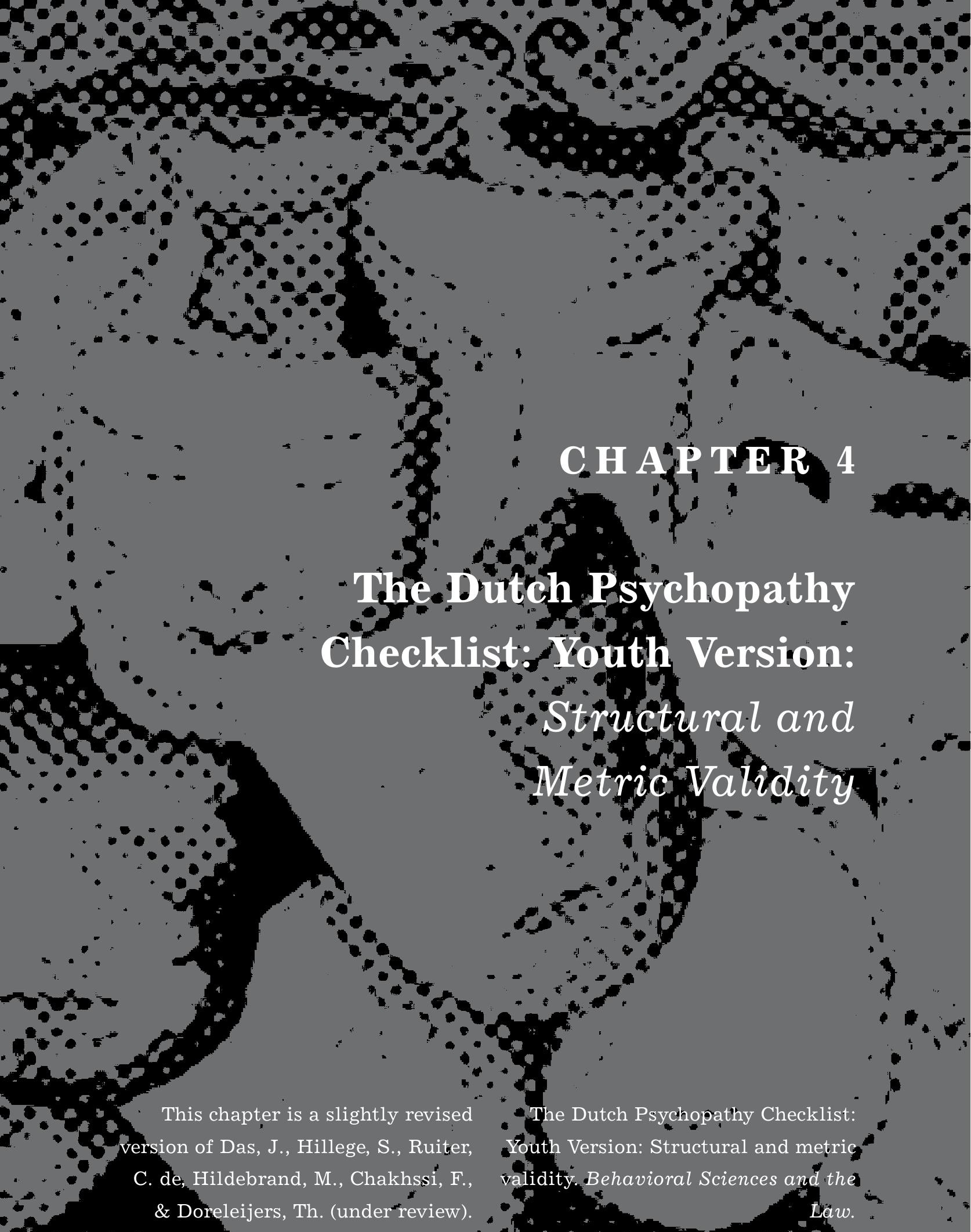




\section{SUMMARY}

In the present study, the structural validity of the Dutch version of the Psychopathy Check List: Youth Version (in Dutch: Psychopathie Checklist: Jeugd Versie; PCL:JV; de Ruiter, Kuin, de Vries \& Das, 2002) in adolescent offenders was examined by means of Confirmatory Factor Analysis (CFA) and Item Response Theory (IRT). The PCL:YV item ratings for 269 adolescent males, either admitted to a juvenile treatment institution in The Netherlands or psychiatrically or psychologically evaluated upon request of the court, were used to test the fit of different factor models that are presumed to represent the structure of psychopathy in adults. The study provides support for a good absolute and relative fit for the hierarchical-, correlated-, and modified three-factor models. Results from the present study do indicate that the traditional two-factor model, and the correlated and modified fourfactor model do not fit properly. Results from IRT analyses demonstrate the highest discriminative power as well as the least susceptibility to age bias for the affective items in the Dutch adolescent sample. Thereby do the present results indicate poor discriminative power and age influence in item functioning in most antisocial factor items.

\section{INTRODUCTION}

The concept of psychopathy has received increasing attention in forensic psychological assessment in the last decades. The Psychopathy ChecklistRevised (PCL-R; Hare, 1991, 2003) has become the gold standard to measure psychopathy in adults. Studies of adult male offenders have demonstrated that the concept of psychopathy is useful in discerning a distinct subgroup, characterized by an early onset of antisocial behavior (Forth \& Burke, 1998; Hare, Hart, Forth, Harpur, \& Williamson, 1998), a deviant performance on neurocognitve tasks (e.g., Newman \& Schmitt, 1998; Levenston, Patrick, Bradley, \& Lang, 2000), a high recidivism rate (Salekin, Rogers, \& Sewell, 1996; Hemphill, Hare, \& Wong, 1998; Hildebrand, de Ruiter, \& de Vogel, 2004), 
and a limited response to treatment efforts (Ogloff, Wong, \& Greenwood, 1990; Rice, Harris, \& Cormier, 1992; Seto \& Barbaree, 1999). Several scholars have argued that the identification of psychopathic traits in childhood or adolescence may offer an understanding of the etiology of the disorder and may provide starting-points for targeting interventions (Forth, Hart, \& Hare, 1990; Forth \& Mailloux, 2000; Frick, Bodin, \& Barry, 2000).

A growing body of research is aimed at examining whether the nomological net surrounding psychopathy in adulthood, also fits juvenile psychopathy (Lynam \& Gudonis, 2005). This research has demonstrated that boys with psychopathic traits show more charges of severe violent and non-violent offenses in the past, more participation in different types of illegal activity in the past and greater willingness to use weapons (Forth et al., 1990; Kosson, Cyterski, Steuerwald, Neumann, \& Walker-Matthews, 2002; Murrie, Cornell, Kaplan, McConville, \& Levy-Elkon, 2004; Salekin, Neumann, Leistico, DiCicco, \& Duros, 2004) than boys without these traits. Furthermore, psychopathy in male adolescent offenders was found to be associated with violent recidivism (Forth et al., 1990; Gretton, Hare, \& Catchpole, 2004; Gretton, McBride, Hare, O’Shaugnessy, \& Kumka, 2001) and a shorter time span between release and re-offense (Brandt, Kenndey, Patrick, \& Curtin, 1997; Gretton et al., 2004). For the assessment of psychopathy in adolescents aged 11-18 years, mainly the Psychopathy Checklist: Youth Version (PCL:YV; Forth, Kosson and Hare, 2003) is often used. The PCL: YV closely resembles the PCL-R, but item descriptions were modified to take into account the restricted life experience of the adolescent and to emphasize peer contacts instead of romantic relationships (Forth et al., 2003). Despite significant parallels between research findings on psychopathy in adults and psychopathy in youths, critics have argued that, due to several methodological problems, the use of a simple downward extension of adult psychopathy measures like the PCL:YV to assess psychopathy in adolescence may not be warranted (Hart, Watt, \& Vincent, 2002). Indeed, since an assessment of psychopathy in the mental health and criminal justice systems may have serious legal implications for an individual (Petrila \& Skeem, 
2003; Skeem \& Cauffman, 2003), the PCL:YV should be the focus of intense psychometric scrutiny. One of the remaining questions in this research is whether the psychopathic traits that define the disorder of psychopathy are the same in adolescents as found in adults (Vincent, 2002). This question can be addressed by evaluating the generalizability of the underlying structure (structural validity) and the score metric (metric validity) of the instrument to the adolescent population.

\section{Structural validity of the PCL:YV}

The dominant model in the PCL-R literature used to be a two factor-structure (Harpur, Hakstian, \& Hare, 1988), wherein Factor 1 is characterized by a selfish and callous interpersonal style and a lack of remorse and empathy, and Factor 2 by a chronically unstable and antisocial lifestyle (Hare et al., 1990). This model could also be demonstrated in adolescent samples (e.g., Brandt et al., 1997; Forth \& Mailloux, 2000). However, Cooke and Michie (2001) have argued that the analytic strategy used in these studies does not justify the two-factor model as the gold standard. More specifically, they criticized the use of the congruence coefficient obtained by using Principal Components Analysis as the only measure of factor similarity. Furthermore, they identified problems with the interpretation of values of congruence coefficients in previous research. Using a bottom-up approach, characterized by a literature review and Confirmatory Factor Analysis (CFA), Cooke and Michie (2001) found support for a hierarchical 13-item three-factor model. In this model, psychopathy is a coherent construct underpinned by three subordinate factors: an arrogant and deceitful interpersonal style (new Factor 1), a deficient affective experience (new Factor 2), and an impulsive and irresponsible behavioral style (new Factor 3 ). The model places little emphasis on criminal behavior, suggesting this should be viewed as a consequence rather than a core feature of psychopathy (Cooke, Michie, Hart, \& Clark, 2004). A comparison between the two- and three factor models in an adolescent sample (Vincent, 2002) also provided support for the three-factor model. Several authors have postulated that exclusion of the criminal items 
is an advance because it avoids tautology when evaluating the relationship between psychopathy and criminal behavior (Farrington, 2005; Johansson, Andershed, Kerr, \& Levander, 2002). In fact, other child and adolescent psychopathy measures, such as the Antisocial Process Screening Device (APSD; Frick \& Hare, 2001) and the Youth Psychopathic traits Inventory (YPI; Andershed, Kerr, Stattin, \& Levander, 2001), do not (YPI) or only sparingly include criminal items (one item; APSD). Nevertheless, several concerns have also been raised regarding the three factor-model. First, a conceptual concern is that the omission of criminal items is arbitrary, taking into consideration that like the lifestyle items, the criminal items are all signs of undercontrolled and externalizing behavior (Neumann, Vitacco, Hare, \& Wupperman, 2005). Furthermore, Hare and Neumann (2005) suggested that rather than criminal behaviors are not merely consequences of psychopathy, this relationship may be interactive and reciprocal in nature. Second, the omission of criminal items may limit the external validity of the PCL:YV (Vitacco, Neumann, \& Jackson, 2005). In fact, Skeem, Mulvey, and Grisso (2003) demonstrated a reduction in the ability to predict future violence of the three-factor model compared to the two-factor model. Third, from a statistical point of view, the three-factor model constitutes a less risky statistical test of model verisimilitude (Neumann et al., 2005). The three-factor model requires ten latent variables to explain the covariance of 13 items. Such a saturated model is likely to result in reasonable fit values. Furthermore, Kosson and colleagues (2002) found in a sample of 115 male adolescents that the hierarchical three-factor model produced impossible values of parameter estimates, whereas a modified model without the testlet-level was found promising. In a re-analysis of the PCL-R validation data, Hare (2003) provided support for a parceled four-factor model underlying the PCL-R, including the three Cooke and Michie (2001) factors and an additional antisocial behavior factor (new Factor 4). Studies comparing the three- and four factor models in adult samples (Hill, Neumann, \& Rogers, 2004; Vitacco et al., 2005) as well as in adolescent samples (Jones, Caufmann, Miller, \& Mulvey, 2006; Neumann, Kosson, Forth, \& Hare, 2006; Neumann, Vitacco, Hare, \& Wupperman, 2005; 
Salekin, Brannen, Zalot, Leistico, \& Neumann, 2006) found both models to have satisfactory fit, but the four-factor model was found to be more parsimonious from a mathematical perspective. Furthermore, the four-factor model was found to be superior to the three-factor model in predicting violence (Hill et al., 2004; Vitacco et al., 2005). It should be noted that instead of using hierarchical models, part of these studies tested intercorrelated models, in which the items loaded directly on the first-order factors without including the superordinate psychopathy factor (Hill et al., 2004; Neumann et al., 2006; Vitacco et al., 2005). In this way, the high level of saturation that is inherent to the hierarchical models is avoided. However, studies (Salekin et al., 2006) that also took the hierarchical three-factor model into account, found a worse fit for this structure, compared to a modified model, due to undefined parameter estimates.

In summary, research findings regarding the structural validity of the PCL-R seem generalizable to the adolescent population. Support was provided for both the three- and four-factor intercorrelated models. However, it remains unclear whether these findings generalize cross-culturally since prior studies are almost exclusively based on North-American samples. 


\section{Metric validity of the PCL:YV}

The generalizability of the score metric of the PCL-R to the adolescent population should be examined in order to determine whether the scoring of the PCL:YV-items is influenced by age. Furthermore, when the effects of age are accumulated across items, this may also influence the total score. The metric properties of a test can be analyzed with Item Response Theory (IRT). When items or tests operate differently for different groups (measurement bias), differential item functioning (DIF) and differential test functioning (DTF) can be demonstrated with IRT analyses. Regarding the PCL-R, IRT has been used to demonstrate an absence of cross-national measurement bias by an invariance of PCL-R scores from Canada versus the United States (Cooke $\&$ Michie, 1997, 2001), and from White versus African American offenders within the United States (Cooke, Kosson, \& Michie, 2001). However, a lack of scalar equivalence across Scottish and North-American criminal offenders was substantial enough to suggest that different PCL-R cut-off scores should be used (Cooke \& Michie, 1999). Recently, Bolt, Hare, Vitale, and Newman (2004) examined DIF and DTF for female criminal offenders, male forensic psychiatric patients, and male criminal offenders scored from file reviews, with a sample of male criminal offenders as a reference group. They found that for the female offender sample, PCL-R items reflecting social deviance were more prone to display DIF than the affective and interpersonal items. Furthermore, despite the presence of a substantial number of DIF items for all comparison groups, the effects in terms of DTF were quite small. Apparently, the influences of external factors cancelled out at the level of total test scores. This was also demonstrated in the only study pertaining to the scalar equivalence of the PCL measures across age. More specifically, Vincent (2002) demonstrated DIF in interpersonal and behavioral items but not in affective items. Again, age bias was found to have little impact at the test-level. 
In summary, IRT provides an appealing framework for studying scalar equivalence of PCL-R assessments in adults in contrast to PCL:YV assessments in adolescents. Preliminary evidence suggests that an age-related measurement bias may exist. More research is needed in order to determine whether these findings can be replicated and generalized to samples other than the North-American population.

\section{The present study}

The first objective of the present study was to examine the cross-cultural generalizability of the factor model underlying the PCL:YV. To this end, the Dutch version of the Psychopathy Checklist: Youth Version (Psychopathie Checklist: Jeugd Versie; PCL:JV; de Ruiter, Kuin, de Vries, \& Das, 2002) was used to measure psychopathic traits in a sample of 269 male adolescents, who were either admitted to a secured juvenile justice treatment institution in The Netherlands or were psychiatrically or psychologically evaluated upon request of the court. Our aim was to identify whether the proposed three- and four-factors model provide good fit to the PCL:YV data in order to determine whether scalar equivalence across cultures is present. The second objective of this study was to evaluate whether psychopathic traits in adolescents as assessed by the PCL:YV define the disorder of psychopathy in the same manner as the PCL-R does in adults. More specifically, IRT techniques were used to examine whether DIF was present in the PCL:YV assessments. 


\section{METHOD}

\section{Participants}

The adolescent sample (total $N=269$ ) comprised three smaller samples from different sites. The first sample comprised 126 male adolescents admitted to a secure section of Jongerenhuis Harreveld and the second sample comprised 66 male adolescents admitted to a semi-secure section of Rentray. Both are juvenile justice treatment institutions for boys who were either sentenced to a supervision order or a mandatory treatment order by the court. A mandatory treatment order is a penal measure which can vary in duration between two and six years, depending on the seriousness of the offense, and on whether a mental disorder was present at the time of the offense. A supervision order is a civil measure which can be imposed when a child's development is psychologically or physically threatened because of incompetent parenting and/or behavioral problems of the child. During the supervision order, the custody of the adolescent becomes shared by the parents and the official child protection agency. The third adolescent sample comprised 93 male adolescents who were a suspect of a violent offense and were psychiatrically and psychologically evaluated upon request of the court in order to determine whether a mandatory treatment order was deemed appropriate. Since the purpose of this study was to assess agerelated measurement bias, potential influences of measurement bias arising from gender were avoided by excluding female participants from the study. Furthermore, sixteen boys (5.6\%) were removed because they were 19 years or older when the PCL:YV was coded, because the PCL:YV was designed for adolescents between the ages 11 and 18 (Forth et al., 2003). In Table 1 an overview is presented of the demographic characteristics of each of the subsamples. The final sample included 269 adolescents with a mean age of 15.70 years $(S D=1.51$; range 11.8 - 19.9). In terms of ethnic origin, $50.4 \%$ was European, $18.1 \%$ was Surinamese, $11.9 \%$ was Moroccan, $4.8 \%$ was from the Netherlands Antilles, and $15 \%$ had another ethnic background. 
Adult PCL-R data were obtained from Hildebrand, Hesper, Spreen, and Nijman (2005). They examined the reliability and (predictive) validity of the PCL-R in 156 males who had been treated in one of eight forensic psychiatric hospitals in The Netherlands under the order of terbeschikkingstelling (tbs). The tbs-order is imposed by court on offenders who have committed a serious violent act and are considered to have diminished responsibility for it because of severe psychopathology. The tbs-order is of indefinite duration: every one or two years the court re-evaluates the patient to determine whether the risk of recidivism is still too high and treatment needs to be continued. Furthermore, additional PCL-R data were obtained from 74 male offenders admitted to the Rooyse Wissel, under the TBS-order. Participants younger than the age of 19 were removed ( $n=2 ; 0.9 \%)$, resulting in a final sample size of 228 male adult offenders. The mean age of the participants in this adult sample was $35.2(S D=8.6$; range $=22-58)$. The ethnic composition was $77.1 \%$ European, 5.4\% Surinamese, 2.7\% Moroccan, 5.4\% Netherlands Antilles, and $9.4 \%$ other.

\section{Psychopathy measures}

In the adult sample, the authorized Dutch translation of the Psychopathy Checklist-Revised (PCL-R; Hare, 1991; Dutch translation: Vertommen, Verheul, de Ruiter, \& Hildebrand, 2002) was used to measure psychopathy. For the adolescent sample, the authorized Dutch translation of the PCL: YV (Psychopathie Checklist: Jeugd Versie; PCL:JV; de Ruiter et al., 2002) manual and scoring sheet were used. For items 9 (Parasitic orientation), 11 (Impersonal sexual behavior), 13 (Lacks goals), 17 (Unstable interpersonal relationships), and 18 (Serious criminal behavior), the descriptive criteria were slightly revised from the Canadian original. Revisions comprised clarifications of when the level of psychopathy symptoms becomes nonnormative. For example, because a certain lack of commitment to long term goals (item 13) is a common part of adolescence, raters were advised to also take into account whether the adolescent had demonstrated commitment to short term goals. Clarifications were added to the item description in 
TABLE 1

DEMOGRAPHIC CHARACTERISTICS OF THE THREE ADOLESCENT SAMPLES

Characteristics

$\begin{array}{rrr}\text { Harreveld } & \text { Rentray } & \text { FPD } \\ \text { sample } & \text { sample } & \text { sample } \\ (n=112) & (n=65) & (n=92)\end{array}$

Age range (years)

13-18

11-18

12-17

Mean age (SD)

16.3

$15.3^{\mathrm{b}}$

$15.3^{b}$

(1.45)

(1.47)

(1.40)

Ethnicity ${ }^{a}$

\% European
$\%$ Surinamese
$\%$ Moroccan
$\%$ Netherlands-Antilles
$\%$ other

64.3

66.2

22.6

10.7

12.3

31.2

6.3

6.2

\footnotetext{
a Significant overall difference between adolescent samples

b Significant difference compared with the Harreveld sample
}

textboxes and Dr. Adelle Forth, first author of the Psychopathy Checklist: Youth Version (Forth et al., 2003), has been informed of these slight amendments.

The 20 items of the PCL-R and PCL:YV are scored on a three point rating scale $(0=$ item does not apply, $1=$ item applies to a certain extent, 2 = item definitely applies), resulting in a dimensional total score ranging from 0 to 40 . Total and factor scores can be prorated when a limited number of items are omitted. For the categorical diagnosis of psychopathy, the traditional cut-off score of 30 was adopted for the PCL-R (Hare, 1991). For the PCL:YV, however, there is no designated cut-off score. According to the 
manual, scores should be interpreted as dimensional ratings of the degree to which an adolescent matches the prototypical psychopath (Forth et al., 2003).

\section{Procedure}

All raters received an official training in the administration and scoring of the PCL-R. The training included a review of the clinical construct of psychopathy and the research literature pertaining to it. Scoring was practiced using videotapes of two Dutch adult forensic psychiatric patients. Raters for the adolescent samples used three additional videotapes of adolescent offenders to practice their scoring.

PCL:YV ratings for the adolescents from both treatment institutions and PCL-R ratings for adults from the Rooyse Wissel were based on the Dutch translation of the semi-structured PCL-R interview designed by Hare (1991; Dutch version: Vertommen, Verheul, de Ruiter, \& Hildebrand, 2002) in conjunction with collateral information, including police files, psychiatric and psychological evaluations and observational reports from previous institutional care. PCL:YV ratings for the adolescents evaluated for the court and PCL-R ratings for the first adult sample were based on file information only. Although the PCL-R and PCL:YV weren't designed to be used without a clinical interview, several studies (e.g., Grann, Långström, N., Tengström, A., \& Stålenheim, 1998; Wong, 1988) have shown that PCL scores derived from extensive file data are reliable and acceptable for research purposes. Single measure Intraclass Correlation Coefficient (ICC; Shrout \& Fleiss, 1979; McGraw \& Wong, 1996) and Cronbach's coefficient $\alpha$ for the PCL:YV total for all subsamples are displayed in Table 3. Overall, figures indicate that the internal consistency of PCL:YV and PCL-R ratings is good enough for basic research applications (Nunnally \& Bernstein, 1994). Furtermore, according to the categories proposed by Fleiss (1986), the observed interrater reliability of the PCL:YV and PCL-R total scores were good to excellent. 


\begin{tabular}{|c|c|c|c|c|c|}
\hline & $\begin{array}{l}\text { Harreveld } \\
\text { sample } \\
(n=108)\end{array}$ & $\begin{array}{c}\text { Rentray } \\
\text { sample } \\
(n=16)\end{array}$ & $\begin{array}{r}\text { FPD } \\
\text { sample } \\
(n=25)\end{array}$ & $\begin{array}{r}\text { Adult } \\
\text { sample } 1 \\
(n=68)\end{array}$ & $\begin{array}{r}\text { Adult } \\
\text { sample } 2 \\
(n=32)\end{array}$ \\
\hline \multicolumn{6}{|l|}{ Single measure ICC } \\
\hline PCL total score & .76 & .74 & .92 & .77 & .77 \\
\hline Factor 1 (Hare, 1991) & .66 & .71 & .52 & .71 & .77 \\
\hline Factor 2 (Hare, 1991) & .67 & .58 & .84 & .73 & .76 \\
\hline New Factor 1 (Hare, 2003) & .68 & .57 & .85 & .67 & .80 \\
\hline New Factor 2 & .41 & .71 & .58 & .55 & .77 \\
\hline New Factor 3 & .61 & .62 & .72 & .63 & .72 \\
\hline New Factor 4 & .71 & .62 & .79 & .77 & .81 \\
\hline Cronbach's $\alpha$ & .82 & .80 & .90 & .83 & .63 \\
\hline
\end{tabular}

\section{Statistical analyses}

The factor structure of the Dutch language version of the PCL:YV was investigated by means Confirmatory Factor Analysis (CFA) in Mplus (Muthen \& Muthen, 1998) in order to determine whether the correlations among items are consistent with the hypothesized three- and four-factor models. Mplus is ideal for testing models composed of ordinal variables, since it uses a robust weighted least squares procedure for parameter estimation and model fit. In order to avoid difficulty in the estimation of fit indices, missing items were replaced by rounded item means equal to 1 . Two absolute fit indices were calculated, including the standardized root mean square residual (SRMR) and the root mean square error of approximation (RMSEA). These indices gauge how well the model-generated covariance matrix reproduces the 
sample matrix. For the RMSEA, values below .08 have traditionally been considered to indicate acceptable fit (Steiger, 1990). More recently, values of approximately .06 and below have been recommended ( $\mathrm{Hu} \&$ Bentler, 1999). The SRMR is expected to be close to or below .08, where sample size exceeds 250 (Hu \& Bentler, 1999). To assess the fit of the hypothesized model with respect to the null-model, the comparative fit index (CFI) and the TuckerLewis index (TLI) were calculated. Values of .94 or above indicate better fit of the hypothesized model (Hu \& Bentler, 1999).

Several versions of the proposed three- and four-factor model were tested, including the hierarchical three-factor model (Cooke \& Michie, 2001; Cooke et al., 2004). Furthermore, in line with previous research (Hill et al., 2004; Jones et al., 2006; Neumann et al., 2005; Neumann et al., 2006; Salekin et al., 2006; Vitacco et al., 2005), correlated models of both the three- and fourfactor model were tested, where items were loaded directly onto the factors and the factors were allowed to intercorrelate. Strong factor inter-relations imply the presence of a higher-order factor (Hill et al., 2004; Neumann et al., 2005; Vitacco et al., 2005). However, in order to determine whether each factor reflects a central feature of a coherent psychopathy construct, additionally both models were tested including a second-order factor. In line with Kosson et al. (2002), these models are referred to as modified models. Finally, also the traditional two-factor model was tested for comparative purposes.

When conducting IRT analyses, unidimensionality is assumed, implying that only one latent trait ( $\theta$; psychopathy) is underlying the testscores. There is a lack of consensus concerning the best method to asses the dimensionality of the PCL:YV and PCL-R. However, if CFA results were to demonstrate good fit of either the hierarchical or modified factor models, this would indicate the presence of a superordinate psychopathy construct, and thus unidimensionality.

MULTILOG VII (Thissen, Chen, \& Bock, 2003) was used to perform IRT analyses. In line with previous research (Bolt et al., 2004; Cooke \& Michie, 1997; Vincent, 2002), Samejima's Graded Response Model (GRM; Samejima, 1997) was used. This three-parameter model is appropriate for tests with 
categorically ordered item ratings that are expected to increase as a function of $\theta$. The interrelation between the probability of a possible response to an item and $\theta$ can be summarized by the parameters $a, b_{1}$ and $b_{2}$. Parameter $a$ is a measure of the discriminating power of an item and is responsible for the slope in so-called item characteristic curves (ICC). The position of the inflection of this slope is given by the thresholds of parameter $b_{1}$ and $b_{2}\left(b_{i}\right)$. Parameter $b_{1}$ is the value of below which the probability of the item being rated 0 is below 0.5 and $b_{2}$ is the value of $\theta$ above which the probability of the item being rated 2 is above 0.5 . The parameter $b_{i}$ provides measures of item extremity or frequency of behavior or attitude (Cooke \& Michie, 1997). Increases in the value of $b_{i}$, represent an increase in the items' level of extremity or infrequency. In order to perform IRT analysis, the data had to be recoded: PCL:YV scores 0, 1 and 2 were recoded as 1, 2 and 3 and missing values were coded as 9 . First, item functioning in the adolescent sample alone is evaluated to describe the relevance of item characteristics to the psychopathy construct in adolescents. Second, differential item functioning (DIF) was investigated. DIF is present when different groups of individuals at similar levels of a common $\theta$ have different probabilities of individual item scores (Hulin, 1987). DIF is present when the parameters of one or more items can't be constrained to be equal across groups without a significant decrease in model fit, as demonstrated by a significant $\mathrm{G}^{2} . \mathrm{G}^{2}$ is distributed as $\chi^{2}$ with $d f$ equal to the number of additional parameter constraints. As long as some test items can be constrained to be equal (anchors), the metric equivalence is sufficient for exploring true item differences. Nonuniform DIF is present if there are significant differences between groups in item slopes ( $a$ parameters) and uniform DIF is present if there are significant differences in item thresholds ( $b$ parameters). 


\section{RESULTS}

\section{Descriptive statistics}

Table 3 provides PCL scores for each sample in accordance with the two-, three- and four-factor model. An independent samples t-test demonstrated that there were no significant differences in mean PCL total- and factor scores between the total adolescent sample and the total adult sample.

\section{Factor structure of the PCL:YV}

Table 4 presents indices of absolute and relative fit for the five models that were tested with CFA. Good absolute and relative fit was demonstrated for the hierarchical-, correlated-, and modified three-factor models. It should be noted, however, that the hierarchical three-factor model resulted in a negative value of the error variance of testlet 6 . The results further indicated that the

TABLE 3

DIFFERENCES IN MEANS AND STANDARD DEVIATIONS OF THE PCL TOTAL- AND FACTOR SCORES OF THE ADOLESCENT SAMPLE AND ADULT SAMPLE

\begin{tabular}{|c|c|c|c|}
\hline $\begin{array}{l}\text { Means } \\
\text { (SD) }\end{array}$ & $\begin{array}{l}\text { Adolescent sample } \\
\qquad(n=269)\end{array}$ & $\begin{array}{l}\text { Adult sample } \\
\qquad(n=228)\end{array}$ & $t(d f)=p=$ \\
\hline PCL total & $21.2(7.8)$ & $20.4(6.9)$ & $t(495)=1.24, p=.22$ \\
\hline Factor 1 & $8.7(3.8)$ & $8.3(3.5)$ & $t(495)=1.11, p=.27)$ \\
\hline Factor 2 & $9.8(3.7)$ & $10.1(4.0)$ & $t(495)=.78 p=.43$ \\
\hline New Factor 1 & $3.0(2.2)$ & $2.8(2.3)$ & $t(493)=1.20 p=.23$ \\
\hline New Factor 2 & $5.6(2.1)$ & $5.6(1.9)$ & $t(490)=.22 p=.83$ \\
\hline New Factor 3 & $5.1(2.5)$ & $5.9(6.6)$ & $t(495)=1.86 p=.06$ \\
\hline New Factor 4 & $5.5(2.3)$ & $5.6(2.7)$ & $t(494)=.77 p=.44$ \\
\hline
\end{tabular}


traditional two-factor model, and the correlated and modified four-factor model, did not have good fit (RMSEA $=.09$ or .10 and CFI $=.86$ or .87 ). Finally, for both the three- and four-factor model, the higher order factor was found to account for the majority of the variance in the interpersonal dimension $\left(\mathrm{R}^{2}=.72\right.$ and .65 , respectively), affective dimension $\left(\mathrm{R}^{2}=.76\right.$ and .74 , respectively), lifestyle dimension $\left(\mathrm{R}^{2}=.75\right.$ and .85 , respectively), and antisocial dimension $\left(R^{2}=.77\right)$, supporting the unidimensional nature of the construct.

TABLE 3

CFA INDICES OF ABSOLUTE AND RELATIVE FIT FOR THE ADOLESCENT SAMPLE

\begin{tabular}{lcccccc}
\hline $\begin{array}{l}\text { Fit } \\
\text { indices }\end{array}$ & $\begin{array}{c}\text { Traditional } \\
\text { two-factor } \\
\text { model }\end{array}$ & $\begin{array}{c}\text { Hierarchical } \\
\text { three-factor } \\
\text { model }\end{array}$ & $\begin{array}{c}\text { Correlated } \\
\text { three-factor } \\
\text { model }\end{array}$ & $\begin{array}{c}\text { Modified } \\
\text { three-factor } \\
\text { model }\end{array}$ & $\begin{array}{c}\text { Correlated } \\
\text { four-factor } \\
\text { model }\end{array}$ & $\begin{array}{c}\text { Modified } \\
\text { four-factor } \\
\text { model }\end{array}$ \\
\hline$\chi^{2}(\mathrm{df})$ & $219.76(63)$ & $79.97(39)$ & $95.37(41)$ & $95.37(41)$ & $209.68(67)$ & $209.06(67)$ \\
$p$ & .00 & .00 & .00 & .00 & .00 & .00 \\
CFI & .86 & .96 & .94 & .94 & .87 & .87 \\
TLI & .92 & .97 & .97 & .97 & .93 & .93 \\
RMSEA & .10 & .06 & .07 & .07 & .09 & .09 \\
SRMR & .09 & .06 & .07 & .07 & .09 & .09 \\
& & & & & & \\
\hline
\end{tabular}

Note CFI = Comparative Fit Index; TLI $=$ Tucker-Lewis index; RMSEA $=$ Root mean square error of approximation; SRMR = Standardized root mean square error .

${ }^{a}$ Model resulted in a negative value of the error variance of testlet 6 . 


\section{Item functioning of the PCL:YV}

In Table 5 the item parameters of the PCL:YV for the adolescent sample are presented. As indicated by the $a$ parameters, three out of four items pertaining to the affective dimension (new Factor 2) were among those with the highest discriminative power, including item 6 (Lack of remorse; $a=$ 2.14), item 8 (Callous or lacking empathy, $a=1.68$ ), and item 16 (Failure to accept responsibility, $a=1.65$ ). Of the interpersonal dimension (new Factor 1), item 1 (Impression management) was found to have poor discrimination ( $a$ $=.91$ ), whereas the discriminative power of the other items was good. Results further demonstrated that the discriminating power of the lifestyle items (new Factor 3) ranged from low (item 14: Impulsivity; $a=.70$ ) to high (item 15: Irresponsibility; $a=1.72$ ). Furthermore, of the antisocial dimension (new Factor 4), only item 20 (Criminal versatility) showed good discrimination. Finally, both items (11: Impersonal sexual behavior and 17: Unstable interpersonal relationships) not belonging to any dimension were among those with the least discriminating power ( $\alpha=.64$ and .87 , respectively).

According to the threshold parameters $b_{1}$ and $b_{2}$, all items discriminated at various levels of the latent trait. However, affective items 6, 8, and 16, lifestyle item 15, and antisocial items 10 and 18 were found to have limited discriminative power at high levels of the latent trait.

\section{Differential item functioning}

A comparison of the statistical fit of the compact model (item parameters are set equal) with the augmented model (parameters are free to vary) indicated the presence of DIF $\left(\mathrm{G}^{2}(60)=744, p<.05\right)$. The following step was to determine whether the DIF in the items was the result of significant differences in item slopes (nonuniform DIF) or item thresholds (uniform DIF). Analyses revealed that nonuniform DIF was present in items 2 (Grandiose sense of self worth), 17 (Unstable interpersonal relationships), 18 (Serious criminal behavior) and 19 (Serious violations of conditional release). As can be seen in Table 6 , these four items were found to have more discriminative power in adolescents than in adults. 
TABLE 5

ITEM PARAMETERS FOR THE ADOLESCENT SAMPLE PRIOR TO COMMON METRIC

PCL:YV Item

1. Impression management

2. Grandiose sense of self-worth

3. Stimulation seeking

4. Pathological Lying

5. Manipulation for personal gain

6. Lack of remorse

7. Shallow affect

8. Callous or lacking empathy

9. Parasitic orientation

10. Poor anger control

11. Impersonal sexual behavior

12. Early behavior problems

13. Lacks goals

14. Impulsivity

15. Irresponsibility

16. Failure to accept responsibility

17. Unstable interpersonal relationships

18. Serious criminal behavior

19. Serious violations of conditional release

20. Criminal versatility a

$\mathrm{b}_{1} \quad \mathrm{~b}_{2}$

.91

$-.27$

1.76

1.46

$-.42$

1.41

1.03

$-.89$

1.02

1.45

$-.26$

1.45

1.59

$-.17$

1.28

2.14

$-1.59$

$-.20$

1.28

$-1.78$

.44

1.68

$-1.78$

.07

1.44

.18

1.64

1.13

$-1.46$

$-.09$

.64

$-.37$

.76

.58

$-1.32$

.83

1.27

$-.86$

1.12

.70

$-2.45$

.39

1.72

$-1.49$

.13

1.65

$-2.04$

$-.24$

.87

$-1.66$

1.51

.82

$-4.58$

$-1.62$

1.00

.73

1.98

1.39

1.07 
TABLE 6

a PARAMETERS FOR ITEMS WITH NONUNIFORM DIF

PCL-R / PCL:YV Item

a parameter

a parameter

$\mathrm{G}^{2}(d f)=\chi^{2}, p$

in adolescent

in adult

sample

sample

2. Grandiose sense of self worth

1.45

.95

$\mathrm{G}^{2}(2)=10.3, p<.05$

17. Unstable interpersonal relationships

.84

.10

$\mathrm{G}^{2}(2)=46.6, p<.05$

18. Serious criminal behavior

.75

.14

$\mathrm{G}^{2}(2)=110.0, p<.05$

19. Serious violations of conditional release

.97

.30

$\mathrm{G}^{2}(2)=26.0, p<.05$

Uniform DIF was found to be present in 15 of 20 items. For all items with uniform DIF, the values of $b_{i}$ are presented in Table 7 . Looking at the pattern of thresholds, it appears that high scores on items 1, 2, 4, 8, 11, 12, 15, 17, and 18 were less frequent in adolescents than in adults. This suggests that significantly higher levels of the latent trait were required for these items to discriminate across adolescents than was required for discrimination across adults. The opposite is true for items $5,9,10,13,19$, and 20 , which required lower levels of the latent trait in order to discriminate across adolescents in comparison to adults. 
TABLE 7

$b_{\text {I }}$ PARAMETERS FOR ITEMS WITH UNIFORM DIF

Adolescent sample Adult sample

PCL-R/PCL:YV Item

1. Impression management

2. Grandiose sense of self-worth

4. Pathological Lying

5. Manipulation for personal gain

8. Callous or lacking empathy

9. Parasitic orientation

10. Poor anger control

11. Impersonal sexual behavior

12. Early behavior problems

13. Lacks goals

15. Irresponsibility

17. Unstable interpersonal relationships

18. Serious criminal behavior

19. Serious violations of conditional release

20. Criminal versatility

$\begin{array}{rrrrrr}a & b_{1} & b_{2} & b_{1} & b_{2} & G^{2}(d f)=\chi^{2}, p \\ .96 & -.32 & 1.65 & .59 & 2.30 & G^{2}(1)=1877, p<.05 \\ 1.24 & -.50 & 1.58 & -.26 & 1.19 & G^{2}(2)=8.6, p<.05 \\ 1.28 & -.35 & 1.55 & .13 & 1.93 & G^{2}(2)=8.9, p<.05 \\ 1.62 & -.13 & 1.35 & -.41 & .84 & G^{2}(2)=10.2, p<.05 \\ 1.57 & -1.95 & .03 & -1.86 & .35 & G^{2}(1)=4.9, p<.05 \\ 1.36 & .23 & 1.78 & -2.07 & .68 & G^{2}(2)=15.2, p<.05 \\ .95 & -1.68 & -.09 & -2.76 & -.38 & G^{2}(2)=13.1, p<.05 \\ .49 & -.44 & .91 & .84 & 2.52 & G^{2}(2)=15.2, p<.05 \\ .51 & -1.53 & .90 & -.48 & 1.54 & G^{2}(1)=7.2, p<.05 \\ 1.12 & -.92 & 1.29 & -1.75 & .56 & G^{2}(2)=20.9, p<.05 \\ 1.47 & -1.73 & .10 & -1.60 & .49 & G^{2}(2)=6.6, p<.05 \\ & & & & & \end{array}$

Note. Parameters in bold were constrained to be equal in both groups. 


\section{DISCUSSION}

In the present study, the structural validity of the Dutch PCL:YV in adolescent offenders was examined by means of Confirmatory Factor Analysis (CFA). Unacceptable fit was indicated for the traditional two-factor model, which is in line with previous research in adolescent samples (Jones et al., 2006; Neumann et al., 2006; Salekin et al., 2006; Vincent, 2002). Regarding the hierarchical three-factor model, our fit indices were similar to those previously demonstrated by Vincent (2002) in an adolescent offender sample, and by Cooke and Michie (2001) in an adult offender sample. However, it was not possible to obtain an error-free test of this model. In line with recent research in adult (Hill et al., 2004; Vitacco et al., 2005) and adolescent samples (Jones et al., 2006; Neumann et al., 2006; Salekin et al., 2006), the correlated three-factor model did result in error-free estimates and provided a good fit to the data. In addition, support was provided for the modified threefactor model proposed by Kosson et al. (2002). Finally, in contrast to previous research (Hill et al., 2004; Jones et al., 2006 Neumann et al., 2005; Neumann et al., 2006; Salekin et al., 2006), neither the correlated nor the modified fourfactor model could be supported by the results from the present study.

Several conclusions can be drawn from these results. First, psychopathy as assessed by the Dutch PCL:YV consists of three dimensions, i.e., an interpersonal, an affective, and a lifestyle dimension. Second, the large degree of variance in these dimensions that could be explained by a superordinate factor, provides evidence for a coherent syndrome, at least at the symptom level. However, Vincent (2002) has suggested that we should only refer to this syndrome as psychopathy after evidence is provided for its stability from adolescence into adulthood. Third, from a model building perspective, the antisocial items may not be necessary features in the assessment of psychopathy in the present sample of male adolescent offenders. Because adolescence shows a peak in antisocial behavior, it may not be as discriminating of psychopathy at this age period, but it may become again in adulthood. In adults the four-factor model has been found to be 
superior to the three-factor model in predicting violence (Hill et al., 2004; Vitacco et al., 2005). Future research should compare the validity of the threeand four-factor model in various populations (e.g., criminal-noncriminal; adult-adolescent; male-female), and their ability to predict aversive outcomes, in order to determine whether antisocial behavior items should be included in the assessment of psychopathy. Furthermore, the validity of both models should also be examined across the periode of adolescence. For instance, Das, de Ruiter, Lodewijks, and Doreleijers (2007) demonstrated that in male adolescents admitted to a secure facility, institutional physical violence was best predicted by the antisocial psychopathy dimension. However, in younger and less criminal adolescents admitted to a semi-secure facility, the lifestyle dimension was more predictive of institutional physical violence. Hypothetically, the lifestyle dimension is more characteristic of psychopathy in younger and (still) less criminal adolescents, and is therefore more likely to be related to physical violence. In a similar vein, the antisocial dimension may be more revealing of psychopathy in older and more criminal adolescents and in adults.

Results from the IRT analyses demonstrated that the affective items were among those with the highest discriminative power and the least susceptibility to age bias, suggesting these items have the most relevance for assessing psychopathy in adolescence. However, they were found to have limited discriminative power at high levels of the latent trait. Considering the importance of the affective dimension, it may be necessary to extend the number of affective items so as to provide more information at higher levels of the latent trait. In this light, Cooke, Hart, and Logan (unpublished manuscript) recently developed the Comprehensive Assessment of Psychopathic Personality disorder (CAPP) for adults. Additionally, item descriptions could also be modified to improve the discriminative power across various levels of the latent trait for each of the four items.

Results further demonstrated that all interpersonal items but item 1 (Impression management) had high discriminative power. Furthermore, uniform DIF was present in three out of the four items. More specifically, 
item 1 (Impression management), item 2 (Grandiose sense of self worth) and item 4 (Pathological lying) were less prevalent among adolescents than among adults. This finding may reflect raters' difficulties in the identification of these traits. As suggested by Vincent (2002) these characteristics may require expression over longer periods of time before they become obvious to raters. At the same time, item descriptions may lack age appropriate descriptions leading raters to overlook the expressions of these characteristics in adolescents. In line with this view, Seagrave and Grisso (2002) argued that the expression of the interpersonal psychopathy characteristics may be influenced by cognitive and social development. For instance, '.. an adolescent may present an insincere and seemingly choreographed social façade, either by attempting to manage peers' impressions or because they are "trying on" a not yet established personality style.. ' (p. 226). The authors suggested that these psychopathic-like traits could be falsely interpreted as features of the psychopathic personality, leading to a higher rate of false positives. In contrast, however, the present results indicate that ambiguity in item descriptions of the phenotypic expressions of the interpersonal characteristics in adolescents may lead to conservative scoring by raters.

Conservative scoring may also have accounted for the low prevalence of a high score on lifestyle item 15 (Irresponsibility). With regard to the lifestyle items Stimulation seeking and Impulsivity results demonstrated that age influence was not present. At the same time, however, these items had poor discriminative power, suggesting they have limited relevance in the assessment of psychopathy. Finally, a high score on item 9 (Parasitic orientation) and 13 (Lacks goals) was more prevalent among adolescents than among adults. It may be that, in contrast to the items $1,2,4,8$, and 15 , these items do include a clear description of the phenotypic expression of these psychopathy characteristics, but they may lack an indication of the level at which this type of behavior becomes nonnormative and indicative of psychopathy. This may lead to false positives in a similar vein as described by Seagrave and Grisso (2002). 
Of the antisocial dimension, items 10 (Poor anger control) and 20 (Criminal versatility) demonstrated moderate discriminative power. The finding that they were more prevalent among adolescents than among adults may also false positive ratings. Furthermore, item 12, 18, and 19 demonstrated poor discriminative power and age influence in item functioning, suggesting they may not be relevant for the assessment of psychopathy in adolescents. This conclusion may also apply to the items 11 (Impersonal sexual behavior) and 17 (Unstable interpersonal relationships), which were also found to show poor discrimination and age influence.

In conclusion, the results from the present study suggest that the affective items have the most relevance for assessing psychopathy in a sample of Dutch adolescent offenders. Furthermore, the interpersonal and lifestyle items were also found to be relevant. However, differential item functioning across age suggests that modifications in item descriptions may be needed. Finally, most antisocial items and the two items that do not load on any dimension, may not be relevant for the assessment of psychopathy in adolescents.

\section{Limitations}

The results of the present study should be qualified by several caveats. More specifically, it is possible that differences in demographic characteristics (i.e., age and ethnicity) and method of psychopathy assessment (i.e., interview and file versus file only) have influenced the results. For instance, cross-national differences in the manifestation of psychopathic traits have been demonstrated by Cooke and Michie 1999). These differences reflect a differential expression of the disorder and do not reflect variations in raters' perceptions of symptoms (Cooke, Hart \& Michie, 2004). Furthermore, Bolt et al. (2004) have found differences in item functioning between PCL-R ratings based on file information versus PCL-R ratings based on a clinical interview, and male PCL-R ratings versus female PCL-ratings. Future research should examine whether these differences are also present among adolescents. Nevertheless, the present study adds to the still limited research base on the 
structural and metric validity of the PCL:YV in adolescent offenders. In the long run, this type of knowledge should help the forensic clinician in gauging the value of PCL-subscores and guide refinement of psychopathy assessment across cultures and ages. 


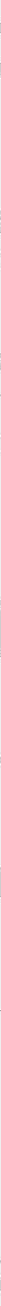

$3 \times 5.2$ $\because$ From Two samples of Mate Treatmentistitution This chapter is a slightly reyised = Institütion. Behavioral Sciences and version of Das, J., Ruiter, C. de, the Law, 2, 739-755. Lodewijks, H., \& Doreleijers, The authors wish to thank all (2007). Predictive Validity of the clinicians who participated in Dutch PCL:YV for Institutional * this study: Special thanks go to Dutch PCL:YV for Institutional
Disruptive Behavior: Findings From $-0$ $+\ldots$ Two Samples of Male Adolescents Kathrin Krämer who contributed to this research during her clinical $-1$ in a Juvenile Justice Treatment internship. .

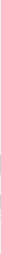




\section{SUMIMARY}

The present prospective study examined the predictive validity of the Dutch version of the Psychopathy Check List: Youth Version for disruptive behavior in male adolescents during treatment. The study comprised two samples admitted to different secure treatment institutions in The Netherlands, Jongerenhuis Harreveld $(\mathrm{n}=81)$ and Rentray $(\mathrm{n}=66)$. Overall, the results demonstrate that psychopathy is a significant predictor of institutional disruptive behavior, and physical violence in specific. Furthermore, Hare's traditional Factor 2 was more strongly related to disruptive incidents than Factor 1. By using the recently proposed three- and four-factor models of psychopathy, insight into the pattern of associations between psychopathy dimensions and different types of disruptive behavior was obtained. The antisocial dimension of psychopathy appeared to be more strongly related to severe incidents than the lifestyle dimension. Regression analyses identified significant contributions of the antisocial and lifestyle dimensions to the prediction of incidents. The relatively small degree of variance explained, underlines the importance of other risk factors to identify adolescents at risk of disruptive behavior during institutional treatment.

\section{INTRODUCTION}

Psychopathy is defined by a set of interpersonal, affective and behavioral traits. A two-factor structure was traditionally thought to underlie the PCL-R (Harpur, Hakstian, \& Hare, 1988; Hare, 1991), in which Factor 1 is characterized by a selfish and callous interpersonal style and a lack of remorse and empathy (Hare et al., 1990), and Factor 2 consists of a chronically unstable and antisocial lifestyle (Hare et al., 1990). The presence of psychopathy in male adult prisoners and forensic psychiatric patients predicts disruptive behavior during imprisonment or hospitalization (see for a review: Edens, Petrila, \& Buffington-Vollum, 2001; Walters, 2003a). Using the Psychopathy Checklist-Revised (PCL-R; Hare, 1991, 2003) or its 
derivatives, researchers have demonstrated that a high psychopathy score is significantly, albeit sometimes modestly, related to institutional infractions (e.g., Belfrage, Fransson, \& Strand, 2000; Edens, Buffington-Vollum, Colwell, Johnson, \& Johnson, 2002; Hildebrand, de Ruiter, \& Nijman, 2004). Guy, Edens, Anthony, and Douglas (2005) recently performed a meta-analysis on 38 independent adult samples (total $N=5,381$ ) and demonstrated that the association between psychopathy and institutional misconduct was strongest for the total number of infractions and for non-violent infractions (e.g., rule violations), and weakest for acts of physical violence. This finding suggests that adult psychopaths undermine the (therapeutic) milieu of their institutional residence and they seem to do this in covert, non-violent ways rather than through overt aggression. However, a moderator effect of nationality was found to be present across all outcome categories indicating smaller weighted mean effect sizes when data were collected in the United States versus data collected in all other countries. For instance, psychopathy was more predictive of physical violence in non-U.S. samples $\left(r_{w}=.21\right)$ than in U.S. samples $\left(r_{w}=.13\right)$.

Only a limited number of studies examined the incremental value of one PCL- $\mathrm{R}$ factor after controlling for the other, by using multiple regression analyses. The significant contribution of Factor 2 in the prediction was consistently found, while there was mixed support for the predictive value of Factor 1 (Walters, 2003a). Recently, new factor models have been proposed for the PCL-R, including a hierarchical three-factor model (Cooke \& Michie, 2001) and a parceled four-factor model (Hare, 2003). In both models, the original Factor 1 (Hare, 1991) is divided into an interpersonal dimension (new Factor 1) and an affective dimension (new Factor 2). A new Factor 3 comprises lifestyle and impulsive behavioral traits. The Hare (2003) four-factor model includes an additional fourth factor comprising antisocial behaviors. These new models allow researchers to further examine the predictive value of specific subdimensions of psychopathy. 
The Psychopathy Checklist: Youth Version (PCL:YV; Forth, Kosson, \& Hare, 2003) is a downward extension of the PCL-R to the adolescent population. In an investigation of the underlying factor structure of the PCL:YV, support was provided for the four-factor model as well as for a modified version of the three-factor model, suggesting that there is considerable continuity in the structure of psychopathy from adolescence to adulthood (Forth et al., 2003).The predictive validity of the PCL:YV for institutional disruptive behavior is receiving increasing interest. A strong significant association $(r=.46)$ between PCL:YV psychopathy scores and institutional charges for misbehavior was demonstrated in a sample of 75 male adolescents in a maximum security center (Forth, Hart, \& Hare, 1990). Furthermore, modest associations of around .30 were found between psychopathic traits and total number of incidents in young adults imprisoned for crimes committed as juveniles (Edens, Poythress, \& Lilienfeld, 1999), in male juvenile offenders in a residential training facility (Brandt, Kennedy, Patrick, \& Curtin, 1997), in a judicial assessment facility (Hicks, Rogers, \& Cashel, 2000), and in a residential treatment program for dually diagnosed offenders (Rogers, Johansen, Chang, \& Salekin, 1997). During the last few years, research into adolescent psychopathy in relation to recidivism is increasing (e.g., Edens, Campbell, \& Weir, 2007; Schmidt, McKinnon, Chattha, \& Brownlee 2006). A meta-analysis across 21 adolescent samples (Edens, Campbell, \& Weir, 2007) has shown a significant association between psychopathy and general, as well as, violent recidivism in adolescents (mean weighted effect sizes of .24 and .25, respectively). Recently, Edens and Campbell (2007) performed a meta-analysis of 15 adolescent samples (total $N=1,310$ ) from thirteen methodologically sound studies, by aggregating effect sizes for the total number of incidents, for a combined category including physical and verbal aggression, and for physical aggression only. The weighted mean effect size for the relation between PCL:YV total scores and total number of incidents was .24. Furthermore, a weighted mean effect size of .28 demonstrated a significant association between psychopathy and institutional physical violence, which is much stronger than the one obtained in the above- 
mentioned meta-analysis with adults reported by Guy et al. (2005). Finally, it was demonstrated that Hare's traditional Factor 2 consistently had greater predictive value than Factor 1. Specifically, the weighted mean effect size for Factor 1 was .21 for the total number of incidents, .22 for the combined category, and .24 for physical aggression, whereas the weighted mean effect sizes for Factor 2 were .28, .34, and .37 for the three categories, respectively.

A growing body of research examines the factors underlying the construct of psychopathy in adolescent samples (e.g. Jones, Cauffman, Miller, \& Mulvey, 2006; Neumann, Kosson, Forth, \& Hare, 2006; Salekin, Brannen, Zalot, Leistico, \& Neumann, 2006; Skeem \& Cauffman, 2003). These studies generally find support for the three- and four-factor models, in favor of the two-factor model. Although conceptually clearer than the four-factor model (Cooke, Michie, \& Skeem, 2007), the hierarchical three-factor model underperforms in terms of predictive power for future recidivism. Results concerning predictive validity have shown that the PCL:YV total score based on the two-factor model was a slightly better predictor of infractions and serious violence during one month of incarceration than the total score based on the three-factor model, suggesting incremental predictive value for the antisocial items. When studying the contribution of the separate factors, Hare's traditional Factor 2 was most predictive of violent infractions and substance abuse, while Hare's traditional Factor 1 score and Cooke and Michie's new Factor 1 score (interpersonal dimension) were most predictive of serious physical violence (Skeem \& Cauffman, 2003).

In summary, the existing evidence suggests a significant, albeit modest, association between psychopathic traits and various forms of institutional disruptive behavior in male adolescent offenders. With regard to the clinical implications of these findings, it has been suggested that the construct of psychopathy may be relevant for purposes of short term risk appraisal and management among juveniles (Edens, Skeem, Cruise, \& Cauffman, 2001). The traditional Hare factors were demonstrated to be differentially related to disruptive behavior while institutionalized. The recently developed threeand four-factor models (Cooke \& Michie, 2001; Hare, 2003) may allow a more 
thorough understanding of these associations. The use of multiple regression analyses is recommended to identify the incremental value of (sub) factors over others (Walters, 2003a).

\section{The present study}

The present study is a prospective study designed to examine the predictive validity of psychopathic traits as measured by means of the PCL:YV for institutional disruptive behavior in adolescent offenders. Because most previous studies in this area were conducted with North American samples, a specific aim was to study whether North American findings can be generalized to adolescents in The Netherlands. The Dutch language version of the PCL: YV (Psychopathie Checklist: Jeugd Versie; PCL:JV; de Ruiter; Kuin, de Vries \& Das, 2002) was used to measure psychopathic traits in male adolescents from two treatment institutions for juvenile offenders. The two samples were analyzed separately in order to identify whether the hypothesized associations are consistent across samples.

1. High PCL:YV total scores are associated with higher frequencies of overall disruptive behavior during institutionalization.

2. High PCL:YV total scores are associated with physical violence during institutionalization.

3. Hare's traditional Factor 2 score is more strongly associated with disruptive behavior during institutionalization, than Hare's traditional Factor 1 score.

4. Factor 4 (antisocial behavior dimension; Hare, 2003) is related to more serious disruptive behavior than Factor 3 (lifestyle behavior dimension; Cooke \& Michie, 2001).

Special attention was directed at identifying the relative contribution of the separate factors (interpersonal, affective, lifestyle and antisocial) of psychopathy in predicting disruptive behavior, and whether the global construct of psychopathy, as defined by the interaction between all factors, made an incremental contribution to the prediction. 


\section{METHOD}

\section{Participants}

The study sample comprised male adolescent offenders from two juvenile justice treatment institutions. Adolescents were either sentenced by court to a supervision order or to a mandatory treatment order. The duration of a mandatory treatment order can vary between two and six years, depending on the seriousness of the offense, on whether a mental disorder was present at the time of the offense and on whether the parenting situation was severely compromised. A supervision order is a civil measure, which can be imposed when a child's development is psychologically or physically threatened because of incompetent parenting and/or behavioral problems of the child. During the supervision order, the custody of the adolescent becomes shared by the parents and the official child protection agency. Adolescents in the first sample were admitted to a secure section of Jongerenhuis Harreveld and are characterized by their involvement in serious criminal behavior. Adolescents in the second sample were admitted to a semi-secure section of Rentray in order to prevent further escalation into antisocial/criminal behavior.

\section{Sample characteristics}

Table 1 presents demographic characteristics for the Harreveld sample and the Rentray sample. Overall, the 66 boys in the Rentray sample were younger $(t=-4.65, p<.01)$, more frequently admitted with a civil measure $\left(\chi^{2}(1)=\right.$ $33.99, p<.01)$ and they had lower mean PCL:YV scores $(t=-4.91, p<.01)$ than the 81 boys in the Harreveld sample. Furthermore, $18(22 \%)$ boys in the Harreveld sample scored 30 or above on the PCL:YV while only two (3\%) boys in the Rentray sample scored 30 or above. Again, this difference was statistically significant $\left(\chi^{2}(1)=11.40, p<.01\right)$. 
TABLE 1

CHARACTERISTICS OF THE HARREVELD SAMPLE $(\mathrm{N}=81)$

AND THE RENTRAY SAMPLE $(\mathrm{N}=66)$

\begin{tabular}{|c|c|c|}
\hline & Harreveld sample & Rentray sample \\
\hline Mean age & $16.7(\mathrm{SD}=1.80)$ & $15.4(\mathrm{SD}=1.58)$ \\
\hline Mean PCL:YV score & $22.78(\mathrm{SD}=6.82)$ & $17.30(\mathrm{SD}=6.65)$ \\
\hline$\%$ PCL:YV > 30 & $22.2 \%$ & $3 \%$ \\
\hline Range PCL:YV total score & $6-36$ & $4-32$ \\
\hline \multicolumn{3}{|l|}{ Ethnic origin } \\
\hline European & $53(65.4 \%)$ & $43(65.1 \%)$ \\
\hline Surinamese & $9(11 \%)$ & $9(13.6 \%)$ \\
\hline Moroccan & $5(6.2 \%)$ & $4(6.1 \%)$ \\
\hline Netherlands Antilles & $3(3.7 \%)$ & $2(3.0 \%)$ \\
\hline Other & $11(13.6 \%)$ & $8(10.6 \%)$ \\
\hline \multicolumn{3}{|l|}{ Judicial measure } \\
\hline Civil supervision order & $36(44.4 \%)$ & $59(89.4 \%)$ \\
\hline Mandatory treatment & $45(55.6 \%)$ & $7(10.6 \%)$ \\
\hline \multicolumn{3}{|l|}{ Index offenses } \\
\hline Violent & $17(37 \%)$ & $2(28.6 \%)$ \\
\hline Sexual & $25(54.3 \%)$ & $3(42.9 \%)$ \\
\hline Property & $2(4.3 \%)$ & $2(28.6 \%)$ \\
\hline Arson & $2(4.3 \%)$ & $0(0 \%)$ \\
\hline
\end{tabular}

Note. PCL:YV = Psychopathie Checklist: Jeugd Versie; SD = Standard deviation. 


\section{Measures}

\section{Psychopathy}

Similar to the PCL-R, the 20 items of the PCL:YV are scored on a three point rating scale $(0=$ item does not apply, $1=$ item applies to a certain extent, 2 = item definitely applies), resulting in a dimensional total score ranging from 0 to 40. Total and factor scores can be prorated when a limited number of items are omitted. For each adolescent, PCL:YV ratings were based on the Dutch translation of the semi-structured PCL-R interview designed by Hare (1991; Dutch version: Vertommen, Verheul, de Ruiter, \& Hildebrand, 2002) in conjunction with collateral information, including police files, psychiatric and psychological evaluations and observational reports from previous institutional care. The authorized Dutch translation of the PCL:YV manual and scoring sheet were used (de Ruiter et al., 2002). For the categorical diagnosis of psychopathy, the traditional cut-off score of 30 was adopted (Hare, 1991). For the items 9 (Parasitic orientation), 11 (Impersonal sexual behavior), 13 (Lacks goals), 17 (Unstable interpersonal relationships), and 18 (Serious criminal behavior), the descriptive criteria were slightly revised from the Canadian original. Revisions comprised clarifications of when the level of psychopathy symptoms becomes non-normative. For example, because a certain lack of commitment to long-term goals (item 13) is a normal part of adolescence, raters were advised to also take into account whether the adolescent has demonstrated to be committed to short term goals. Clarifications were added to the item description in textboxes and Dr. Adelle Forth, first author of the Psychopathy Checklist: Youth Version (Forth et al., 2003), has been informed of these revisions. The psychometric properties of the PCL:YV have been discussed elsewhere (Das, de Ruiter, Doreleijers, \& Hillege, accepted). The reliability of the PCL:YV total score was acceptable, although less than the one reported in the original PCL:YV manual (Forth et al., 2003). Furthermore, the interrater reliability for the individual items and the four separate factors was also somewhat lower than what is reported in the PCL:YV manual, which is in line with previous findings by Spain, Douglas, Poythress, and Epstein (2004). 


\section{Incidents}

A classification scheme for rating of institutional infractions designed by Hildebrand et al. (2004) was slightly modified to assign incidents to a certain category. The scheme includes four categories: (1) Verbal abuse (inappropriate verbal expressions, cursing), (2) Verbal threat (verbal hostility with the intention to psychologically harming another person or threatening to act physically violent), (3) Physical violence (violent behavior against people or property), and (4) Violation of institutional rules (including use of drugs, unauthorized absence, positive urinalysis results). For the Harreveld sample, the registration of incidents was based on TULP (a judicial system for the registration of client information), on internal memos, and on daily reports written by group leaders and teachers. Two raters (the first author and a trained undergraduate psychology student) independently reviewed 113 daily reports by group leaders to examine agreement on whether the events reported, qualified as incidents. The observed agreement was $96.5 \%$ and Cohen's $\kappa$ was .80 , indicating excellent agreement. Subsequently, 100 incidents were randomly selected from the total number of incidents and coded by the two independent raters with regard to type of incident. Because there was excellent agreement (observed agreement $=92 \%$; Cohen's $\kappa=.88$ ), further ratings were made by one independent rater. For each adolescent, the total number of incidents in each category was divided by the number of days spent in the institution, in order to correct for the fact that the time at risk of institutional misbehavior was not equal for participants.

For the Rentray sample, two independent raters used half-yearly treatment evaluation reports written for the court and the before mentioned TULP system for the registration of incidents. Excellent interrater reliability (Cohen's $\kappa=.96$ ) was demonstrated for 110 randomly selected incidents, justifying further single ratings by an independent research assistant. 


\section{Disciplinary actions}

For both samples, TULP was used to count two types of disciplinary actions, i.e., seclusion in a designated room and placement in another institution for juvenile offenders for the duration of two weeks (correctional placement). Again, the total number of disciplinary actions was corrected for the length of institutional stay.

\section{Procedure}

All raters received training in the administration and scoring of the PCL-R by the second author and/or a mental health professional, who had been trained by Drs. Robert D. Hare and David Cooke. The training included a review of the clinical construct of psychopathy and the research literature pertaining to it. Scoring was practiced using videotapes of two Dutch adult forensic psychiatric patients and three adolescent offenders. In the current study, most adolescents were interviewed and rated by the supervising psychologist of the ward he was admitted to. The PCL-R interviews were videotaped after written informed consent by the adolescent and informing the parents about the purpose and procedure of the study. For the Harreveld sample, PCL:YV ratings were conducted by 14 raters; ten female and four male, and for the Rentray sample by two female raters. Two independent ratings were available for $74(91.4 \%)$ boys in the Harreveld sample and for 16 boys $(24.2 \%)$ in the Rentray sample.

\section{Data-analysis}

The interrater reliability of the PCL:YV was examined by means of the intraclass correlation coefficient (ICC), using a two-way random effects model and the consistency type (McGraw \& Wong, 1996). The observed interrater reliability was evaluated according to the following categories: $\mathrm{ICC} \geq .75=$ excellent; $.60 \leq$ ICC $<.75=$ good; $.50 \leq$ ICC $<.60=$ moderate; ICC $<.50=$ poor (Fleiss, 1986).

Spearman $\rho$ correlations instead of Pearson correlations were calculated between PCL:YV total and factor scores and all outcome variables 
because of the ordinal nature of the PCL:YV data. In addition, the total number of incidents was not normally distributed in both the Harreveld sample (Kolmogorov-Smirnov $Z=1.52, p=.02$ ) and the Rentray sample (Kolmogorov-Smirnov $Z=1.65, p=.02$ ).

Linear regression with the stepwise procedure (based on the $F$ statistic) was conducted to determine which psychopathy dimension(s) were significant predictors of the total number of incidents and the different incident categories. Furthermore, psychopathy as defined by the interaction between all four psychopathy factors was entered in block 2 , to identify whether it had incremental value to the prediction. The criterion for entry was set at .05 and for removal at .10 .

\section{RESULTS}

\section{Reliability}

Table 2 presents the single measure ICCs for the PCL:YV total- and factor scores in the Harreveld and Rentray samples. Overall, the interrater reliability of the PCL:YV total score and Hare's traditional Factor 1 and 2 was good in both samples. Furthermore, interrater reliabilities for the interpersonal, lifestyle, and antisocial dimensions were adequate, whereas the affective dimension of psychopathy had poor interrater reliability in the Harreveld sample.

Cronbach's coefficient $\alpha$ for the PCL:YV total score indicated high internal reliability for both samples (.81 and .80 for the Harreveld and Rentray sample, respectively). The mean inter-item correlation was .18 for the Harreveld sample and .16 for the Rentray sample.

\section{Frequency of disruptive behavior}

Table 3 presents the frequency of disruptive behavior in the Harreveld and Rentray samples. In comparison to the Rentray sample, boys in the Harreveld sample were followed for a shorter period of time $(t=-4.33, p<.01)$, but 
TABLE 2

INTRACLASS CORRELATION COEFFICIENTS (ICCS) SINGLE MEASURE OF PCL:

YV TOTAL- AND FACTOR SCORES IN THE HARREVELD SAMPLE $(\mathrm{N}=74)$ AND THE

RENTRAY SAMPLE $(N=16)$

PCL:YV

$\begin{array}{lrrrr}\text { Total } & \text { Hare's } & \text { Hare's } & \text { Inter- Affective } & \text { Lifestyle Antisocial } \\ & \text { Factor } 1 & \text { Factor } 2 & \text { personal }\end{array}$

$\begin{array}{lccccccc}\text { Harreveld Sample } & .75 & .63 & .67 & .56 & .45 & .58 & .74 \\ \text { Rentray Sample } & .74 & .71 & .58 & .57 & .71 & .62 & .62\end{array}$

Note. PCL:YV = Psychopathie: Checklist: Jeugd Versie (Psychopathy Checklist: Youth Version). All ICCs were significantly greater than $0(\mathrm{p}<.05)$.

they displayed significantly more incidents of verbal abuse $(t=3.02, p<.01)$ and physical violence $(t=3.73, p<.01)$ during their stay in the institution. At the same time, the average number of incidents per adolescent is highly similar in both samples (19.4 and 21.3 for the Harreveld sample and the Rentray sample, respectively).

\section{PCL:YV scores in relation to disruptive behavior}

Table 4 presents the Spearman $\rho$ correlations between PCL:YV total and factor scores and all outcome variables. In the Harreveld sample, significant relations demonstrated that the PCL:YV total score was predictive of the total number of incidents $(\rho=.37)$, and the incident categories verbal abuse ( $\rho=.25)$, physical violence $(\rho=.34)$, and rule violations $(\rho=.39)$. Hare's traditional Factor 1 score was only found to be significantly related to correctional placement $(\rho=.19)$, whereas the strongest correlations were 
TABLE 3

FREQUENCY OF DISRUPTIVE BEHAVIOR IN THE HARREVELD SAMPLE

AND RENTRAY SAMPLE

Harreveld sample

1512

Total number of incidents

Average follow-up period in days

Average number of incidents per

adolescent

Incident category

Verbal abuse

Verbal threat

Physical violence

Violation of institutional rules

Self-harm

Disciplinary action

Seclusion

Correctional placement

Adolescents not involved in any

type of incident

Note. $\mathrm{SD}=$ Standard deviation

found between Hare's traditional Factor 2, the total number of incidents ( $\rho=$ $.44)$, and rule violations $(\rho=.45)$.

Evaluating the lifestyle dimension and the antisocial dimension separately, a different pattern of correlations was identified. In particular, the lifestyle dimension was more strongly related to rule violations $(\rho=$ $.41)$, whereas the antisocial dimension was more strongly related to total number of incidents $(\rho=.35)$, verbal abuse $(\rho=.36)$, physical violence ( $\rho$ 
$=.36)$, and correctional placement $(\rho=.26)$. Although no hypothesis was formulated with regard to self-harm, the results demonstrated a trend with the interpersonal dimension $(\rho=.19)$.

In the Rentray sample, only modest but significant associations were found for physical violence, including an association with the PCL:YV total score $(\rho=.29)$, Hare's traditional Factor $2(\rho=.29)$, and the lifestyle dimension $(\rho=.28)$. Furthermore, the antisocial dimension was associated with seclusion $(\rho=.25)$. Finally, the relation between the interpersonal dimension and self-harm $(\rho=.28)$ could also be identified in this sample. In general, however, much fewer significant associations between the PCL:YV and institutional infractions were found in the Rentray sample, compared to the Harreveld sample.

\section{The relative contribution of psychopathy dimensions in the prediction of disruptive behavior}

In the Harreveld sample, linear regression analysis demonstrated that the antisocial dimension was the only variable to enter the equation in the prediction of the total number of incidents $\left(R^{2}=.07, p<.05\right)$. Psychopathy, as defined by the interaction term of all four factors, did not produce a significant improvement to the model fit $(F=.32, p=n s)$. Similarly, the antisocial dimension was a significant predictor of verbal abuse $\left(R^{2}=.05\right.$, $p<.05)$ but the interaction term did not improve the model fit $(F=.01$, $p=n s)$. For physical violence, the individual psychopathy dimensions as well as the interaction term failed to enter the equation. Finally, the lifestyle dimension was the only variable to enter the equation in the prediction of rule violations $\left(R^{2}=.12, p<.01\right)$. Again, the interaction term did not improve the model fit ( $F=.01, p=n s$ ). Regarding seclusion, none of the PCL:YV factors, nor the interaction term entered the equation in the prediction of seclusion. The interaction term, but none of the factors, significantly predicted correctional placement $\left(R^{2}=.10, p<.01\right)$. 
TABLE 4

SPEARMAN $\rho$ CORRELATIONS BETWEEN PCL:YV SCORES AND INCIDENTS

Type of Incident

$\begin{array}{rrrrrr}\text { PCL:YV } & \text { Factor } 1 & \text { Factor } 2 & \text { Inter- Affective } & \text { Lifestyle } & \text { Anti- } \\ \text { total } & \text { (Hare, } & \text { (Hare, personal } & \text { social } \\ & 1991) & 1991) & & \end{array}$

\section{Harreveld sample}

Total number of incidents

$\begin{array}{lcccccc}.37 * * & .12 & .44 * * & .08 & .08 & .33 * * & .35 * * \\ .25 * & .10 & .28 * * & .11 & .05 & .11 & .36 * * \\ .15 & .08 & .18 & .02 & .04 & .11 & .18 \\ .34 * * & .09 & .39 * * & .01 & .08 & .25 * & .36 * * \\ .39 * * & .17 & .45 * * & .17 & .09 & .41 * * & .27 * * \\ .09 & .09 & .01 & .19 & -.06 & -.00 & .07 \\ .19 * & .03 & .25 * & -.05 & .14 & .23 * & .14 \\ .27 * * & .19 * & .25 * & .08 & .17 & .15 & .26 * * \\ & & & & & & \end{array}$

\section{Rentray sample}

$\begin{array}{rrrrrrr}-.07 & -.07 & -.05 & -.01 & -.08 & .08 & .05 \\ .11 & .11 & .05 & .06 & .10 & .03 & .06 \\ .10 & .02 & .18 & .03 & .02 & .10 & .13 \\ .29 * & .20 & .29 * & .13 & .21 & .28 * & .18 \\ -.08 & -.05 & -.07 & -.01 & -.07 & -.06 & .01 \\ .17 & .17 & .17 & .28 * & .08 & .11 & .13 \\ .05 & .01 & .03 & -.06 & .04 & .04 & .06 \\ .23 & .13 & .24 & .14 & .09 & .24 & .25 *\end{array}$

$* \mathrm{p}<.05$, two-tailed.

$* * \mathrm{p}<.01$ level, two-tailed. 
In the Rentray sample, significance was only achieved by the antisocial dimension for predicting correctional placement $\left(R^{2}=.09, p<.05\right)$. The interaction term did not improve model fit $(F=2.21, p=n s)$.

\section{DISCUSSION}

The present study comprised two samples of boys admitted to different juvenile justice treatment institutions. The samples differed in terms of age, the proportion of boys placed under a criminal justice measure as opposed to a civil supervision order, and mean psychopathy scores. This provided an opportunity to examine possible differences in predictive power of the construct of psychopathy as measured with the PCL: Youth Version for institutional disruptive behavior. In both samples, a high base rate of incidents was found, suggesting disruptive behavior during treatment in juvenile justice institutions is a substantial problem. In fact, in the present study the average number of incidents per adolescent was higher than in previous studies with adult offender samples (e.g., Edens et al., 2002; Hildebrand et al., 2004), and adolescent offender samples (e.g., Edens et al., 1999; Hicks et al., 2000).

\section{Psychopathy total scores in relation to institutional disruptive behavior}

The first hypothesis postulated that high PCL:YV total scores are associated with higher frequencies of overall disruptive behavior during institutionalization. In line with previous studies (Brandt et al., 1997; Edens et al., 1999; Forth et al., 1990; Hicks et al., 2000; Rogers et al., 1997), this hypothesis was supported in the Harreveld sample by a correlation of .37. In the Rentray sample, however, this association could not be demonstrated. Although the variance of PCL:YV scores was similar for both samples (Harreveld sample: $S D=6.82$; Rentray sample: $S D=6.65$ ), PCL:YV total scores were consistently lower in the Rentray sample. As opposed to $22 \%$ in the Harreveld sample, only $3 \%$ of the Rentray boys had a PCL:YV total score of 30 or more. The fact that few adolescents in the Rentray sample had 
elevated psychopathy scores may have resulted in attenuated correlations. The results may imply that psychopathy at the low end of the distribution is not particularly useful in terms of differentiating adolescents prone to institutional misbehavior and those not so prone.

The second hypothesis that high PCL:YV total scores are related to physical violence was supported in both the Harreveld and the Rentray sample, which is in line with previous research in adolescents (see the metaanalysis by Edens \& Campbell, 2007). Thus, psychopathy may be qualified as a robust predictor of physical violence during institutionalization, regardless of the degree of psychopathy of the sample under investigation. The results further demonstrated that the association between psychopathic traits and the other incident categories (verbal abuse, verbal threat, rule violations) are dependent on the sample under investigation. To be more specific, in the Harreveld sample where psychopathic traits were highly prevalent, the presence of these traits is associated with various types of disruptive behavior. However, when psychopathic traits are less prevalent as in the Rentray sample, the construct may not be useful for identifying adolescents at risk of incidents other than physical violence. In such a sample, an evaluation of risk factors other than psychopathy may be critical. In fact, [reference to co-authors, under review) demonstrated that in the Rentray sample the SAVRY (Structured Assessment of Violence Risk in Youth; Borum, Bartel, \& Forth, 2002) was useful in predicting physical violence as well as other incident categories, including rule violations and verbal threat. The systematic assessment of a constellation of historical, social, and individual risk factors combined with protective factors proved to be a useful method for identifying youth at risk of displaying various forms of disruptive behavior while institutionalized.

\section{Dimensions of psychopathy in relation to institutional disruptive behavior} Hare's traditional two-factor model (Hare, 1991) and the recently proposed three- and four-factor models (Cooke \& Michie, 2001, and Hare, 2003, respectively) were useful in examining the pattern of associations between 
the separate psychopathy dimensions and disruptive behaviors. The third hypothesis that Hare's traditional Factor 2 is a more effective predictor of disruptive behavior than Factor 1, was supported in both samples. Furthermore, partial support was provided for the fourth hypothesis stating that the antisocial dimension is related to more serious misbehavior than the lifestyle dimension. The antisocial dimension was most strongly related to correctional placement in both samples, implying that this dimension of psychopathy is predictive of the most serious forms of disruptive behavior resulting in the most severe disciplinary action. Inconsistent with the hypothesis, however, both dimensions were found to be related to mild as well as serious incident categories. It is important to note that physical violence was best predicted by the antisocial dimension in the Harreveld sample, whereas in the Rentray sample it was best predicted by the lifestyle dimension. This finding may perhaps be explained in light of the crossnational (Cooke, Hart and Michie, 2004), gender-related (Bolt, Vitale, Hare, \& Neumann, 2004; Schmidt et al., 2006) and age-related (Vincent, 2002) differences in the expression of psychopathy that have been demonstrated in previous research. Hypothetically, the lifestyle dimension is more characteristic of psychopathy in younger and (still) less criminal adolescents such as the Rentray sample, and is therefore more likely to be related to external correlates of psychopathy such as physical violence. In a similar vein, the antisocial dimension may be more revealing of psychopathy in older and more criminal adolescents such as the Harreveld sample. The lifestyle dimension may be an important precursor to antisocial behavior, which is expressed in the person's criminal lifestyle over time.

Considerable debate exists about which symptoms form the core symptoms of psychopathy. For instance, Cooke, Michie, Hart, and Clark (2004) have argued that the interpersonal, affective, and impulsive lifestyle dimensions are core symptoms, while antisocial symptoms should be perceived as consequences of psychopathy. In contrast, others have found that antisocial tendencies also play a fundamental role in the assessment of psychopathy (Hare, 2003; Neumann, Vitacco, Hare, \& Wupperman, 2005). 
Research findings demonstrating differential correlations between individual psychopathy factors and external criteria, highlight the importance of each of these factors (e.g., Hall, Benning, \& Patrick, 2004; Vitacco, Neumann, \& Jackson, 2005). In this study, only the behavioral dimensions were found to have predictive value for (general) institutional externalizing behavior. This is in line with Walters' (2003a) statement that “...behavioral models appear to have an advantage over personality models (Cleckley, 1941 / 1976; Hare, 1996) in the sense that behavioral models closely approximate the behavioral criteria used to gauge disciplinary adjustment...”(p. 543). Similarly, the antisocial dimension was also found to be the "active ingredient" in the association between the psychopathy and recidivism in six forensic / correctional adult samples (Walters, Knight, Grann, \& Dahle, 2008). The antisocial dimension had incremental validity relative to the other three dimensions. However, the lifestyle dimension was unique in distinguishing violent and nonviolent recidivists, again suggesting that the behavioral components of psychopathy are most helpful in predicting future misbehavior.

Nevertheless, it is still possible that the interpersonal and affective dimensions are related to the broader concept of treatment response, including program retention, treatment module attendance and therapist ratings of participation. For instance, Hobson and Shine (2000) have demonstrated that Hare's traditional Factor 1 (and not Factor 2) was significantly associated with negative behavior during therapy groups and community meetings, and with general negative behavior on the wing. More recently, Richards, Casey, and Lucente (2003) found Hare's traditional Factor 1 to be related to infractions during treatment, new charges in the community after treatment, but also to a shorter stay in treatment and removal from the program due to rule violations. In the only Dutch study pertaining to this subject, Hare's traditional Factor 1 was not found to be associated with treatment compliance (Hildebrand, de Ruiter, \& van Zaane, in preparation). However, in this study only one aspect of treatment compliance was measured, i.e. attendance rate of therapeutic activities. In all, these findings 
suggest that the interpersonal and affective dimensions may be related to more subtle indicators of treatment response than the ones used in the present study.

\section{The relative contribution of psychopathy dimensions}

Regression analyses were performed in order to evaluate the relative contribution of the PCL:YV factor- and total scores in the prediction of institutional disruptive behavior. Again, the relatively superior contribution of the lifestyle and antisocial dimension was demonstrated in the Harreveld sample. Furthermore, the global construct of psychopathy, as defined by the interaction between the four factors, only contributed significantly in the prediction of correctional placement. A correctional placement is only imposed after a very serious incident has occurred, which means this finding suggests psychopathic traits predict the most severe forms of disruptive behavior. This is in line with previous research demonstrating that while the PCL:YV total score did not prove predictive of (mild) violent/aggressive incidents, it was predictive of serious violence during incarceration (Skeem \& Cauffman, 2003). Finally, the regression analyses in the Rentray sample demonstrated limited relevance of a high PCL:YV score in the prediction of institutional disruptive behavior, suggesting other risk factors should be considered when psychopathy has a low base rate in a sample.

\section{Psychopathic traits and self-harm}

The present study also showed a moderate positive association between the interpersonal dimension and self-harm, suggesting adolescents with a glib, grandiose, and manipulative interpersonal style more frequently engage in self-harm than adolescents not showing this interpersonal style. It should be noted, however, that the base rate of self-harming behaviors in both samples was rather low, increasing the chance of spurious findings. In previous studies of adult male and female samples, Hare's traditional Factor 1 (including the interpersonal and affective dimension) was found to be either negatively related or unrelated to parasuicidal behavior (Gray et 
al., 2003; Verona, Hicks, \& Patrick, in press; Verona, Patrick, \& Joiner, 2001). Moreover, the negative correlation between Factor 1 and suicide attempts was determined by the interpersonal dimension of psychopathy (Verona et al., in press). Self-harm is mainly thought to be related to internalizing forms of psychopathology, such as anxiety and depression. Thus, the negative association between parasuicidal behavior and the interpersonal dimension found in adult samples is in line with the evidence that shows that lack of internalizing psychopathology is a core feature of psychopathy (Hildebrand, 2004; Skeem, Mulvey, \& Grisso, 2003). In adolescent samples, however, PCL: YV scores have been found to be either unrelated (Brandt et al., 1997; Skeem \& Cauffman, 2003) or even positively related with negative affect (Kosson et al., 2002), suggesting adolescent psychopathy may be associated with greater negative affectivity than psychopathy in adulthood. Kosson and colleagues (2002) postulated that 'Whereas psychopathic adults have a seamless veneer of affectlessness, adolescents with psychopathic traits have the same dispositions (impulsivity, callousness, manipulativeness, etc.) but have not yet developed the same impenetrable mask of sanity' (p. 106). Consistent with this hypothesis, the positive association between the interpersonal dimension of psychopathy and self-harm was demonstrated to be stronger in the younger and less criminal Rentray sample than in the older and more criminal Harreveld sample. More research with different kinds of measures of negative emotionality (including self-harm) is needed to improve our understanding of its association with adolescent psychopathy.

\section{Limitations}

The results of the present study should be qualified by several caveats. First and foremost, since the focus of the study was to evaluate the predictive validity of the Dutch PCL:YV and the dimensions within the concept of psychopathy, no alternative predictor variables were included in the analyses. The relatively small degree of variance explained by PCL:YV total- and factor scores, should prompt researchers to study other relevant risk factors in youth at risk for institutional disruptive behavior. Second, although much 
effort was devoted to applying uniform procedures for data gathering in both samples, practical circumstances led to differences in a) the sources that were used for the registration of incidents and $b$ ) the procedure for examining interrater reliability of the incident registration. Despite close monitoring of the coding procedures, any potential influence on the results cannot be ruled out.

\section{Conclusion}

In line with previous North American findings, the Dutch PCL:YV was proven a significant predictor of disruptive behavior in adolescents during institutional treatment in the juvenile justice system (Brandt et al., 1997; Edens et al., 1999; Forth et al., 1990; Hicks et al., 2000). This association is primarily determined by the behavioral dimensions and not the personality dimensions of psychopathy. The PCL:YV total score proved to be useful in the prediction of the most serious incidents. The results underline the importance of considering other relevant predictor variables when trying to identify adolescents at risk for displaying institutional disruptive behavior, especially in populations where psychopathic traits are less prevalent. 

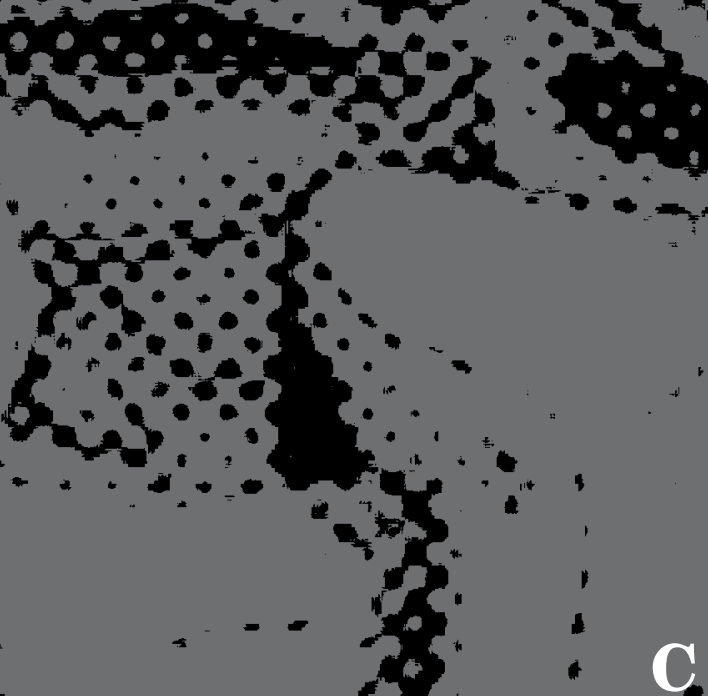

$a$

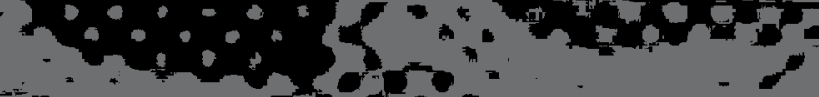

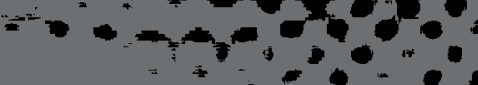

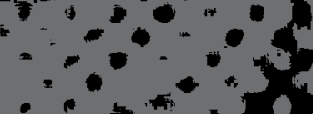

$=$

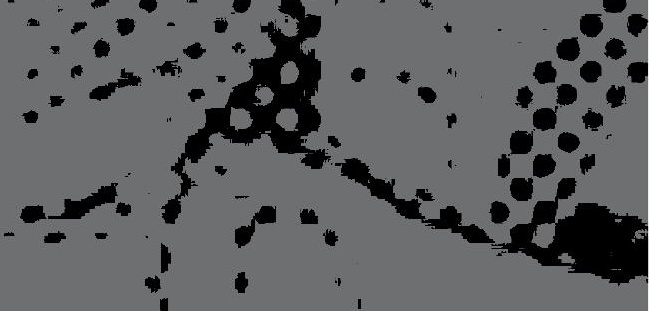

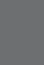

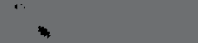

The 10

The Youth fesychopathic Traits $\because$ ine -1

Inventorysychometric

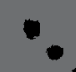
$\therefore \cdots$

A 3 Properties and Its Relation to

$\because \therefore$. Sutance Use and Substance Use andinterpersonal $\because$ Style in a Dutch sample of H Non-referwed Ad This chapter is bašed on Hillege, "Style, in a Dutch Sample of NonS., Das, J., \& Ruiter, C. de (under. Traits Inventory: Psychometric "The authors wish to thank Voke de Traits Inventory: Psychometric "The authors wish to thank Voke de Traits Inventory: Psychometric "The authors wish to thank Voke de $-$ Properties and Its Relation to Boer who contributed to this research $+\cdots$ Substance Use and Interpérsonal during her clinical internship.

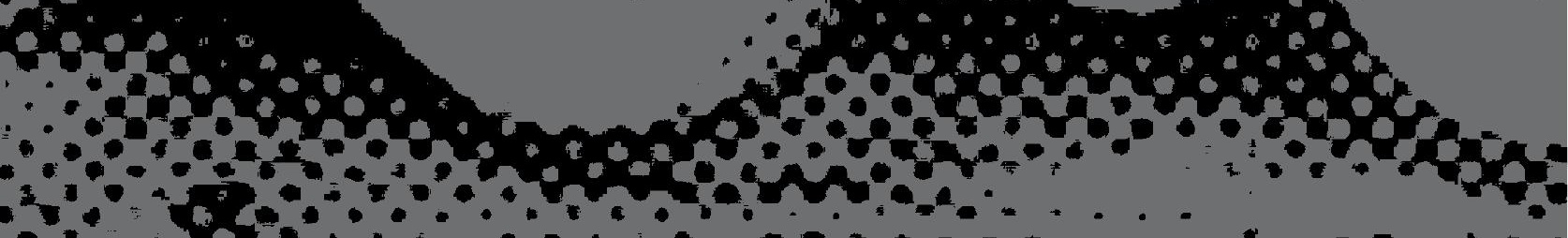




\section{SUMMARY}

This study presents evidence on the reliability and construct validity of the Dutch version of the Youth Psychopathic Traits Inventory (YPI). In a sample of 776 Dutch non-referred adolescents, the YPI was found to have good internal consistency. Furthermore, exploratory Principal Components Analysis (PCA) indicated a three factor structure for the YPI, including a Grandiose-manipulative, Callous-unemotional, and Impulsiveirresponsible dimension. Results further indicated strong correlations between psychopathic traits and dominant and hostile interpersonal styles. The Grandiose-manipulative dimension predicted dominant interpersonal behavior in boys, but not in girls. In girls, a dominant interpersonal style was predicted by the Impulsive-irresponsible dimension. Psychopathic traits, mainly the Impulsive-irresponsible dimension, were related to a higher frequency of self-reported drug and alcohol use, in boys and girls.

\section{INTRODUCTION}

The interest in the concept of psychopathy has broadened in the past decades. Although extensive research has shown that knowledge and descriptions of psychopathic character traits in adult male offenders are also applicable to the younger subset of male adolescent offenders (e.g., Brandt, Kennedy, Patrick, \& Curtin, 1997; Forth, Hart, \& Hare, 1990; Jones, Cauffman, Miller, \& Mulvey, 2006; Neumann, Kosson, Forth, \& Hare, 2006; Vincent, 2002), the assessment of psychopathic traits in children and adolescents remains subject to debate (Edens, Skeem, Cruise, \& Cauffman, 2001; Seagrave \& Grisso, 2002). Nevertheless, it has also been argued that the identification of psychopathic traits early in life might offer a better understanding of the etiology of the disorder and its conceptualization (Farrington, 2005; Forth et al., 1990). Better insight into the development of violent, persistent and criminal behavior can of course stimulate initiatives for preventive treatment intervention (Andershed, Kerr, Stattin, \& Levander, 2001). 
A growing body of research focuses on the nomological network surrounding adolescent psychopathy and its commonality with psychopathy in adulthood (Benning, Patrick, Blonigen, Hicks, \& Iacono, 2005; Benning, Patrick, Salekin, \& Leistico, 2005; Lynam, Caspi, Moffit, Raine, Loeber, \& StouthamerLoeber, 2005; Loney, Taylor, Butler, \& Iacono, 2007; Salekin, Leistico, Trobst, Schrum, \& Lochman, 2005). The nomological network is a theoretical framework, which provides understanding of the construct validity of a measure (Cronbach \& Meehl, 1955). In this framework, constructs are continually redefined and clarified through their relationships with other constructs. The nomological net surrounding psychopathy consists of three levels: 1) the convergence between measures of psychopathy, 2) the relationship between psychopathy measures and measures of personality disorders, and 3) the relationship between psychopathy measures and measures of normal-range personality dimensions (Benning, Patrick, Salekin et al., 2005).

\section{Assessment of psychopathic traits in adolescents}

Originally, research on psychopathic traits in youth has focussed on adolescents who had been involved in criminal acts using the Psychopathy Checklist: Youth Version (PCL:YV; Forth, Kosson, \& Hare, 2003). This downward extension of the Psychopathy Checklist-Revised (PCL-R; Hare, $1991,2003)$ is a time consuming method in assessing psychopathic traits in adolescents, and extensive training and forensic experience is required. Furthermore, several critics have argued that the use of a simple downward extension of adult psychopathy measures may not be warranted because of several methodological and ethical problems (Hart, Watt, Vincent, \& 2002; Seagrave \& Grisso, 2002). Other instruments to measure psychopathic traits in children and adolescents such as the Antisocial Process Screening Device (APSD; Frick \& Hare, 2002) and the Child Psychopathy Scale (CPS; Lynam, 1997) do not remediate these shortcomings. For instance, 1) the transparency of the ASPD items increases the chance of response bias (Poythress, Dembo, Wareham, \& Greenbaum 2006) and 2) only one item is used to measure each 
psychopathic trait (Andershed, Gustafson, Kerr, \& Stattin, 2002).

Taking into account these shortcomings, The Youth Psychopathic Traits Inventory (YPI; Andershed et al., 2001) was developed. The YPI was designed to measure the interpersonal (Grandiose-manipulative), affective (Callous-unemotional) and lifestyle (Impulsive-irresponsible) dimensions of psychopathy and focuses on 10 core traits of psychopathy in community youth (Andershed et al., 2001). The main purpose of the YPI is to measure personality traits, rather than behavioral traits related to psychopathy. The authors argue that behavioral characteristics associated with psychopathy do not apply to the same extent to non-referred youths, and that these characteristics do not seem to form the core of the psychopathy construct (Andershed et al., 2001). Guiding principles in the development of the YPI were: (1) each psychopathic trait should be measured with several items so that analyses can be done on the subscale level; (2) psychopathic traits should be measured indirectly, rather than directly, and (3) the items should be worded so that the traits sound positive to a person with psychopathic traits, but not to those without them. Since the YPI was developed as a measure for community youth, one of its advantages is that the instrument enables researchers to study the nomological network of psychopathic traits in adolescents in the general population. Furthermore, the self-report items of the YPI possibly provide a more accurate insight into the experiential aspects of psychopathy, which cannot be assessed by parent or teacher report (Andershed et al., 2002; Frick, Barry, \& Brodin, 2000). Finally, because it is a self-report measure, it is cost- and time effective (Andershed et al., 2002).

Prior research has demonstrated that the YPI is internally consistent and a useful measure for delineating a psychopathic-like subgroup of nonreferred boys and girls with antisocial and behavioral problems (Andershed et al., 2001; 2002). Furthermore, exploratory and confirmatory factor analysis support a 'theoretically meaningful and useful' three-factor structure (Andershed et al., 2001, p. 151), similar to the three-factor structure found for the PCL-R (Cooke \& Michie, 2001) and PCL:YV (Jones et al., 2006; Neumann et al., 2006; Salekin, Brannen, Zalot, Leistico, \& Neumann, 2006). 
In institutionalized young offenders, the YPI was found to have good testretest reliability, to be related to short-term institutional misbehavior, and to have a theoretically coherent inverse relationship with anxiety (Skeem \& Cauffman, 2003). Furthermore, the Impulsive-irresponsible dimension showed positive correlations with thrill seeking, impulsiveness and irresponsibility (Dolan \& Rennie, 2007) and was most strongly predictive of short-term institutional infractions (Skeem \& Cauffman, 2003). In all, these findings suggest that the YPI is a promising self-report measure of psychopathic traits in adolescents.

\section{Psychopathy and personality dimensions}

Concerning the second level of the nomological network surrounding psychopathy, it has been suggested that the concept of psychopathy can be described as a malicious conceptualization of extremes of normal personality traits (e.g., Benning, Patrick, Salekin et al., 2005; Widiger \& Lynam, 1998). Research has demonstrated that effects of psychopathic traits on an individual's interpersonal style can be mapped around the interpersonal circumplex model (Benning, Patrick, Salekin et al., 2005; Salekin et al., 2005). In the interpersonal circumplex, interpersonal styles are organized around two opposing axes: Dominance and Affection (Benning, Patrick, Salekin et al., 2005). Interpersonal theory suggests that individuals show a distinctive interpersonal style resulting from personality traits and developmental experience, with an emphasis on one of the octants of the circumplex (Salekin et al., 2005). Strong correlations between psychopathic traits and a dominant interpersonal style were demonstrated in a sample of juvenile offenders (Salekin et al., 2005). Similarly, self-report psychopathy scores from an undergraduate student sample were associated with a dominant interpersonal style (Benning, Patrick, Salekin et al., 2005).

\section{Substance abuse and psychopathy}

Research in adult samples (Hemphill, Hart, \& Hare, 1994; Smith \& Newman, 1990; Walsh, Allen, \& Kosson, 2007), as well as adolescent samples (Mailloux, 
Forth, \& Kroner, 1997; O’Neill, Lidz, \& Heilbrun, 2003) has demonstrated a positive relation between psychopathic traits and substance use. This relation is demonstrated to be reciprocal in nature and to be primarily determined by the impulsive and irresponsible lifestyle dimension (Harvey, Stokes, Lord, \& Pogge, 1996; Mailloux et al., 1997; Taylor \& Lang, 2006; Walsh et al., 2007). Adolescent males with high PCL:YV scores had an earlier onset of substance use, a wider variety of drug use, and higher scores on self-report screening tests for substance use than those who had lower scores (Mailloux et al., 1997). Furthermore, recent prospective research in non-referred male adolescents has shown significant predictive value of psychopathy scores for (future) substance use (Loney et al., 2007). This study, using a self-report version of the Minnesota Temperament Inventory (MTI; Loney, Taylor, Butler, \& Iacono, 2002), showed that features from the lifestyle dimension were unique in predicting substance symptoms.

Using the YPI in a sample of referred adolescents, significant relations were demonstrated between the dimensions Grandiose-manipulative and Impulsive-irresponsible and severity of drug use in the past year (Poythress, Dembo, Wareham, \& Greenbaum, 2006). Results from structural equation modeling indicated that the Impulsive-irresponsible factor was primarily responsible for the significant correlation with past drug use. Using the YPI in a non-referred sample of adolescents, Andershed et al. (2002) demonstrated a positive association between psychopathic traits and drug use as well.

\section{The present study}

The present study examines the psychometric properties of the Dutch translation of the YPI in a sample of non-referred adolescents. Analyses were conducted separately for boys and girls, since studies report gender differences in PCL:YV (Andershed, Hodgins, \& Tengström, 2007) and YPI scores (Andershed et al., 2002). The internal consistency and factor structure, using exploratory Principal Components Analysis (PCA), were studied. Further, scores on the YPI were related to scores on self-report instruments designed to measure interpersonal behavioral styles and alcohol and drug 
use. Special attention was directed at identifying the relative value of the separate dimensions of the YPI in the associations with the other measures. On the basis of previous research, strong associations were expected between psychopathic traits on the one hand, and a dominant and hostile interpersonal style and substance use, on the other hand. With regard to the separate dimensions of psychopathy, the Impulsive-irresponsible dimension was expected to be largely responsible for the association between psychopathic traits and self-reported alcohol and drug use.

\section{METHOD}

\section{Subjects}

The sample comprised 776 adolescents in the upper grades of secondary school. Thirty-six adolescents (5\%) did not complete the YPI and were removed from the sample. The final sample included 740 adolescents with a mean age of 15.6 years $(S D=.94$; range 14-19). Forty-seven percent of the sample was male (47\%), $53 \%$ was female. The majority of the sample was Dutch (88.4\%), while $12 \%$ had another ethnic origin (e.g., Somalian, Turkish, Netherlands-Antillean).

\section{Instruments}

\section{YPI}

The authorized Dutch translation of the Youth Psychopathic traits Inventory (YPI; Das \& de Ruiter, 2003) was used to measure psychopathic traits. The YPI is a 50-item self-report measure. The applicability of the items is rated on a 4 -point Likert scale ( 1 = Does not apply at all, 2 = Does not apply well, 3 = Applies fairly well, 4 = Applies very well). In the instruction, it is stressed that there are no right or wrong answers and that for each item the adolescent should consider what he or she generally thinks or feels and not how he or she feels at that moment. The YPI consists of 10 subdimensions designed to capture the core traits of psychopathy. These 
subdimensions pertain to either the Grandiose-manipulative, the Callousunemotional or the Impulsive-irresponsible dimension. More specifically, the Grandiose-manipulative dimension consists of the subdimensions Dishonest charm (e.g., "When I need to, I use my smile and my charm to use others"), Grandiosity (e.g., "I am more important and valuable than other people"), Lying (e.g., "Sometimes I lie for no reason, other than because it's fun"), and Manipulation (e.g., "To get people to do what I want, I often find it efficient to con them"). Furthermore, the Callous-unemotional dimension consists of the subdimensions Remorselessness (e.g., "I seldom regret things I do, even if other people feel that they are wrong"), Unemotionality (e.g., "What scares others usually doesn't scare me"), and Callousness (e.g., "When other people have problems, it is often their own fault, therefore, one should not help them"). Finally, the subdimensions Thrill seeking (e.g., "I get bored quickly by doing the same thing over"), Impulsiveness (e.g., "I prefer to spend my money right away rather than save it"), and Irresponsibility (e.g., "I have cut classes more than most other people"), belong to the Impulsive-irresponsible dimension. In line with previous research (Andershed et al., 2001; 2002; Skeem \& Cauffman, 2003), average continuous YPI total- and dimensionscores were used in the analyses.

\section{Interpersonal Checklist-Revised}

The authorized Dutch translation of the Interpersonal CheckList-Revised (ICL-R; de Jong, van den Brink, \& Jansma, 2000) was used to map interpersonal behavioral styles onto the interpersonal circumplex. The ICL$\mathrm{R}$ is a renewed version of the Interpersonal CheckList (LaForge \& Suczek, 1955) and based on Leary's (1957) model of interpersonal behavior. The ICL-R consists of 160 items, which are rated yes/no. In the current study, item scores were prorated in case of two or less missing items. The instrument contains ten behavioral dimensions, which indicate whether the interpersonal style is predominantly characterized by dominance versus submissiveness, and by hostility versus friendliness. In previous research with the Dutch ICL-R, the intercorrelations and the circumplex analysis supported the hypothesized 
circular arrangement of the interpersonal styles (De Jong et al., 2000). The test-retest reliability of the interpersonal styles is moderate to good (ICC's range from .57 to .83; de Jong et al., 2000). For the current sample, good internal consistency was found for total ICL-R scores (Cronbach's alpha $=$ $.84)$.

\section{Drug Use Disorders Identification Test}

A Dutch version of the Drug Use Disorders Identification Test (DUDIT; Berman, Bergman, Palmstierna, \& Schlyter, 2002; Dutch version: Das \& de Ruiter, 2003) was used for the screening of drug use disorders. The DUDIT is constructed to offer simplicity in identifying drug related problems. It contains 11 items, which provide information on different facets of drug use, such as frequency and poly drug use. The items are rated on a 3- or 5- point interval scale. In the current study, item scores were prorated in case of two or fewer missing items. The DUDIT was validated in several samples of heavy drug users, as well as in the general population (Berman et al., 2005) and was found to be internally consistent in both samples (Cronbach's alpha $=.80$ and .93 , respectively). The internal consistency for the present sample was excellent (Cronbach's alpha $=.92$ ).

\section{Screening of Alcohol Use}

In co-operation with Tactus, an institute for treatment of addiction, the authors constructed a screening instrument to measure frequency and reasons for drinking alcohol. The instrument for the Screening of Alcohol Use (SAU) consists of nine items, which can be rated on a 3- or 5-point continuous interval scale (e.g. "How often did you drink an alcoholic beverage in the last four weeks?"). The structure of the SAU is similar to the structure of the DUDIT. As in the ICL-R and DUDIT, scores were prorated in the case of two or fewer missing items. The internal consistency of the SAU in the present sample was acceptable (Cronbach's alpha $=.65$ ). 


\section{Procedure}

The instruments were administered to 776 adolescents in the upper grades of two secondary schools, located in the north and in the south of The Netherlands. The questionnaires were completed within one hour in the classroom under the supervision of a teacher. A standardized introduction to the tests was given and a glossary was used when the adolescents asked for explanation of a term used in one of the questionnaires. The subjects were informed that their participation was voluntary and that their responses would be processed confidentially and anonymously.

\section{Statistical analyses}

The first set of analyses aimed at providing descriptive information concerning the distribution of YPI ratings in the sample. Student's $t$-test was used to test mean group differences in YPI scores between male and female subjects and Dutch and non-Dutch subjects. In addition, internal consistency (Cronbach's alpha) was calculated for the YPI total score, the three dimensions, and the ten subdimensions. To explore the factor structure of the Dutch YPI, exploratory Principal Components Analysis (PCA) with oblique Promax rotation was performed. The construct validity of the YPI was examined by calculating Pearson correlations between YPI total- and original dimensional scores, and scores on the ICL-R, DUDIT, and SAU. All statistical analyses were performed in SPSS 13.0 for Windows. 


\section{RESULTS}

\section{Descriptive characteristics}

Cronbach's alpha coefficients indicated acceptable internal consistencies for the three main dimensions and the YPI total score in boys and girls (Table 1). Internal consistencies for the subdimensions were largely acceptable, with the exception of Unemotionality $(\alpha=.51)$ and Callousness $(\alpha=.32)$ in boys, and Callousness $(\alpha=.52)$ in girls.

\footnotetext{
TABLE 1

INTERNAL CONSISTENCY FOR THE TEN SUBDIMENSIONS AND

THREE DIMENSIONS OF THE YPI IN BOYS $(N=341)$ AND GIRLS $(N=387)$
}

\begin{tabular}{lll}
\hline YPI Subdimensions and Dimensions & Alpha Boys & Girls \\
\hline Dishonest charm & .76 & .78 \\
Grandiosity & .82 & .74 \\
Lying & .70 & .76 \\
Manipulation & .78 & .78 \\
Remorselessness & .68 & .63 \\
Unemotionality & .51 & .60 \\
Callousness & .32 & .52 \\
Thrill-seeking & .62 & .71 \\
Impulsiveness & .60 & .72 \\
Irresponsibility & .60 & .62 \\
Grandiose-manipulative dimension & .84 & .82 \\
Callous-unemotional dimension & .66 & .60 \\
Impulsive-irresponsible dimension & .71 & .78 \\
Total Score & .70 & .74 \\
& & \\
\hline
\end{tabular}

Note. YPI = Youth Psychopathic traits Inventory. 
Table 2 contains means and standard deviations for the subdimensions, dimensions and total YPI score for boys and girls separately. An independent samples t-test, demonstrated significantly higher scores for boys on all but one subdimension (Impulsiveness; $t(123)=1.23, p=.22$ ). An examination of potential differences in YPI scores due to ethnicity, demonstrated a significantly higher score on the Grandiosity subdimension for nonDutch participants $(t(701)=-2.47, p=.02)$. Since scores on the other (sub)dimensions did not reveal significant differences between subjects of Dutch and Non-Dutch ethnic origin, we did not control for ethnicity in further analyses.

TABLE 2

DESCRIPTIVE STATISTICS FOR THE SUBDIMENSIONS, DIMENSIONS AND TOTAL SCORE OF THE YPI AMONG BOYS $(\mathrm{N}=341)$ AND GIRLS $(\mathrm{N}=387)$

\begin{tabular}{|c|c|c|c|c|c|}
\hline Subscale & Gender & $\mathrm{N}$ & Mean & $\mathrm{SD}$ & $\begin{array}{c}t(\mathrm{df})= \\
p=\end{array}$ \\
\hline \multirow[t]{2}{*}{ Dishonest charm } & Boys & 331 & 1.98 & .68 & $t(705)=5.21$ \\
\hline & Girls & 376 & 1.73 & .62 & $p=.00$ \\
\hline \multirow[t]{2}{*}{ Grandiosity } & Boys & 320 & 1.84 & .75 & $t(692)=10.24$ \\
\hline & Girls & 374 & 1.34 & .46 & $p=.00$ \\
\hline \multirow[t]{2}{*}{ Lying } & Boys & 330 & 1.96 & .63 & $t(705)=7.10$ \\
\hline & Girls & 377 & 1.64 & .60 & $p=.00$ \\
\hline \multirow[t]{2}{*}{ Manipulation } & Boys & 325 & 1.96 & .68 & $t(700)=8.23$ \\
\hline & Girls & 377 & 1.57 & .57 & $p=.00$ \\
\hline \multirow[t]{2}{*}{ Remorselessness } & Boys & 326 & 1.97 & .63 & $t(695)=9.43$ \\
\hline & Girls & 371 & 1.56 & .50 & $p=.00$ \\
\hline \multirow[t]{2}{*}{ Unemotionality } & Boys & 328 & 2.21 & .54 & $t(695)=13.02$ \\
\hline & Girls & 369 & 1.70 & .49 & $p=.00$ \\
\hline
\end{tabular}




\begin{tabular}{|c|c|c|c|c|c|}
\hline Table 2 (continued) & Gender & $\mathrm{N}$ & Mean & $\mathrm{SD}$ & $\begin{array}{c}t(\mathrm{df})= \\
p=\end{array}$ \\
\hline \multirow[t]{2}{*}{ Callousness } & Boys & 327 & 2.48 & .48 & $t(695)=15.94$ \\
\hline & Girls & 371 & 1.88 & .51 & $p=.00$ \\
\hline \multirow[t]{2}{*}{ Thrill-seeking } & Boys & 327 & 2.75 & .61 & $t(701)=6.14$ \\
\hline & Girls & 376 & 2.46 & .63 & $p=.00$ \\
\hline \multirow[t]{2}{*}{ Impulsiveness } & Boys & 321 & 2.32 & .61 & $t(686)=1.23$ \\
\hline & Girls & 367 & 2.26 & .66 & $p=.22$ \\
\hline \multirow[t]{2}{*}{ Irresponsibility } & Boys & 328 & 1.80 & .61 & $t(698)=5.81$ \\
\hline & Girls & 372 & 1.55 & .53 & $p=.00$ \\
\hline \multirow[t]{2}{*}{ Grandiose-manupulative } & Boys & 291 & 7.72 & 2.28 & $\mathrm{t}(638)=8.93$ \\
\hline & Girls & 349 & 6.25 & 1.81 & $\mathrm{p}=.00$ \\
\hline \multirow[t]{2}{*}{ Callous-unemotional } & Boys & 304 & 6.66 & 1.28 & $\mathrm{t}(649)=15.91$ \\
\hline & Girls & 347 & 5.16 & 1.11 & $\mathrm{p}=.00$ \\
\hline \multirow[t]{2}{*}{ Impulsive-irresponsible } & Boys & 297 & 6.86 & 1.44 & $\mathrm{t}(639) 5.21$ \\
\hline & Girls & 344 & 6.25 & 1.53 & $\mathrm{p}=.00$ \\
\hline \multirow[t]{2}{*}{ Total YPI score } & Boys & 244 & 21.24 & 4.12 & $t(540)=10.42$ \\
\hline & Girls & 298 & 17.72 & 3.64 & $\mathrm{p}=.00$ \\
\hline
\end{tabular}

Note. YPI = Youth Psychopathic traits Inventory.

\section{Factor structure}

In the male sample, Principal Component Analysis (PCA) with oblique rotation of the ten subdimensions revealed three factors with eigenvalues greater than one, accounting for $66 \%$ of the total variance. Although the three-factor structure was theoretically interpretable, three subdimensions (Lying, Remorselessness and Callousness) loaded above .30 on more than one factor. Exploratory PCA in the female sample demonstrated two factors with eigenvalues greater than one, accounting for $56 \%$ of the total variance. Three subdimensions (Dishonest charm, Lying, and Irresponsibility) loaded more 
than .30 on both factors. Extending this model with a third factor resulted in a model quite similar to that in boys, with the only difference that the subscale Lying clearly loaded on the interpersonal dimension in the sample of girls. The factor solutions for both boys and girls are presented in Table 3 .

TABLE 3

YOUTH PSYCHOPATHIC TRAITS INVENTORY FACTOR OBLIQUE SOLUTIONS (PATTERN MATRIX) FOR A SAMPLE AMONG ADOLESCENT BOYS $(\mathrm{N}=341)$ AND GIRLS $(\mathrm{N}=387)$

Boys Girls

$\begin{array}{lrrrrrr}\text { YPI Subdimensions } & \text { G-M } & \text { C-U } & \text { I-I } & \text { G-M } & \text { C-U } & \text { I-I } \\ \text { Dishonest charm } & .93 & -.23 & -.04 & .79 & -.14 & .04 \\ \text { Grandiosity } & .92 & .06 & -.20 & .77 & .20 & -.16 \\ \text { Lying } & .46 & .05 & .33 & .75 & -.22 & .12 \\ \text { Manipulation } & .87 & -.03 & .02 & .96 & -.11 & -.09 \\ \text { Remorselessness } & .35 & .50 & .19 & .39 & .23 & .26 \\ \text { Unemotionality } & .32 & .54 & .07 & .48 & .38 & .03 \\ \text { Calloussness } & -.26 & .96 & -.13 & -.13 & .95 & -.04 \\ \text { Thrill-seeking } & -.17 & -.03 & .85 & -.08 & -.03 & .90 \\ \text { Impulsiveness } & -.04 & -.09 & .86 & -.06 & -.08 & .88 \\ \text { Irresponsibility } & .08 & .00 & .73 & .26 & .16 & .57 \\ & & & & & & \end{array}$

Note. G-M = Grandiose Manipulative, $\mathrm{C}-\mathrm{U}$ = Callous- Unemotional, $\mathrm{I}-\mathrm{I}$ = Impulsive -Irresponsible. All factor loadings > .30 are in bold. 


\section{Construct validity}

\section{Interpersonal style}

Table 4 presents correlations between the original Andershed et al. YPI dimension- and total scores and scores on the ICL-R. In boys, the YPI total score was significantly positively related to a dominant and hostile interpersonal style, while significantly inversely related to a submissive and friendly behavioral style. Regarding the separate dimensions, all three YPI dimensions were significantly positively related to a dominant and hostile interpersonal style (Table 4). These correlations were strongest for the Grandiose-manipulative dimension $(r=.30)$. Furthermore, all three YPI dimensions showed significant inverse relationships with a submissive and friendly interpersonal style, with the strongest relation for the Callousunemotional dimension $(r=-.41)$.

Similar to boys, YPI total scores in girls were found to be significantly positively related to a dominant and hostile interpersonal style $(r=$ .25), while significantly inversely related to a submissive and friendly interpersonal style $(r=-.22)$. The patterns of association between the three main dimensions of psychopathy and ICL-R scores in girls were quite similar to those found in boys. However, a notable difference was that in girls the Impulsive-irresponsible dimension was most strongly related to a dominant and hostile interpersonal style, while in boys it was the Grandiosemanipulative dimension. 
TABLE 4

CORRELATIONS BETWEEN YPI DIMENSIONS, AND TOTAL SCORES ON THE ICL-R, DUDIT AND SAU IN BOYS $(N=341)$ AND GIRLS $(N=387)$

YPI

\begin{tabular}{lrrrrr}
\hline Interpersonal style & Total & Grandiose- & Callous- & Impulsive- \\
ICL-R & & manipulative & unemotional & irresponsible
\end{tabular}

\begin{tabular}{|c|c|c|c|c|c|}
\hline \multirow[t]{2}{*}{ Dominance } & Boys & $.28 * *$ & $.30 * *$ & $.11 *$ & $.15^{* *}$ \\
\hline & Girls & $.25 * *$ & $.21 * *$ & $.12 * *$ & $.25 * *$ \\
\hline \multirow[t]{2}{*}{ Affiliation } & Boys & $-.28 * *$ & $-.17 * *$ & $-.41 * *$ & $-.13 * *$ \\
\hline & Girls & $-.22 * *$ & $-.22 * *$ & $-.24 * *$ & $-.13 *$ \\
\hline \multirow[t]{2}{*}{ DUDIT } & Boys & $.20 * *$ & $.21 * *$ & $.17 *$ & $.20 * *$ \\
\hline & Girls & $.24 * *$ & $.21 * *$ & $.20 * *$ & $.13 * *$ \\
\hline \multirow[t]{2}{*}{ SAU } & Boys & $.24 * *$ & $.21 * *$ & $.18 * *$ & $.28 * *$ \\
\hline & Girls & $.27 * *$ & $.16 * *$ & $.10 *$ & $.35 * *$ \\
\hline
\end{tabular}

Note. YPI $=$ Youth Psychopathic traits Inventory, ICL-R = Interpersonal Checklist-Revised, DUDIT

$=$ Drug Use Disorder Identification Test, SAU $=$ Screening of Alcohol Use, $* \mathrm{p}<.05$, two-tailed $* * \mathrm{p}<.01$, two-tailed

\section{Substance use}

Correlations between YPI dimension- and total scores and DUDIT and SAU scores are presented in Table 4. In boys, a significant association was demonstrated between the YPI total score, drug use disorder symptoms and frequency of alcohol use. Furthermore, all three YPI dimensions showed significant positive correlations with the DUDIT and SAU total scores, with the Impulsive-irresponsible dimension showing somewhat stronger associations. In girls, YPI total scores were significantly positively related 
to drug use disorder symptoms and frequency of alcohol use. All three dimensions were significantly related to the number of drug use symptoms, while only the Grandiose-manipulative and Impulsive-irresponsible dimension were associated with frequency of alcohol use.

\section{DISCUSSION}

\section{Reliability of the YPI}

This study was the first to evaluate the psychometric properties of the YPI in a Dutch sample. In line with previous studies in community youth (Andershed et al., 2001), and clinical samples (Poythress et al., 2006; Skeem \& Cauffman, 2003) the present results provide support for the internal consistency of dimension- and total scores of the YPI. For the subdimensions Unemotionality $(\alpha=.50)$ and Callousness in boys $(\alpha=.32)$, and the Callousness subdimension in girls $(\alpha=.52)$ reliability was poor. Previous studies in clinical samples have also found poor reliability indices for the Callousness subdimension $(\alpha=.36$; Poythress et al., 2006; $\alpha=.49$; Skeem \& Cauffman, 2003). The present findings strengthen previous suggestions that affective psychopathic characteristics are less well assessed by means of selfreport instruments, because of their resistance to reliable description (Hall, Benning, \& Patrick, 2004). Hall and colleagues (2004) concur that this is due to the lack of insight psychopathic individual have into their emotional deficits. Furthermore, the affective items may be sensitive to response bias and social desirability (Poythress et al., 2006). Since a deficient affective experience is generally considered to be at the core of the psychopathy construct (Cooke \& Michie, 2001; Farrington, 2005; Vincent, 2002), a revision of the Callousness subdimension of the YPI is recommended.

\section{Factor structure}

The three factors that were extracted with exploratory factor analysis in the present sample were quite similar to the Grandiose-manipulative, Callous-unemotional and Impulsive-irresponsible dimensions, proposed by 
Andershed and colleagues (2001). Furthermore, and in line with previous findings (Andershed et al., 2001), the subdimensions Remorselessness and Unemotionality were found to load on all three main YPI dimensions in the female and male subsample. These findings may reflect the nature of the interrelationships between the separate psychopathy dimensions. Specifically, the finding that the subscales Remorselessness and Unemotionality load on all three factors, may support the notion that a deficient affective experience forms the core of the psychopathy syndrome (Cooke \& Michie, 2001; Farrington, 2005; Vincent, 2001). Further research should use confirmatory factor analysis and item response theory.analysis in order to further examine the nature of the interrelationships between psychopathy dimensions.

\section{Construct validity}

Similar to what was found in incarcerated adolescents (Salekin et al., 2005), the YPI total- and dimension scores demonstrated strong associations with a dominant and hostile interpersonal style, and significant inverse relations with a submissive and friendly interpersonal style. Furthermore, in line with previous findings in incarcerated (Mailloux et al., 1997) and non-referred samples (Andershed et al., 2002; Loney et al., 2007), the current findings indicate that adolescent psychopathic traits are strongly associated with substance abuse problems. However, some gender differences on the psychopathy dimensions were identified. Specifically, in boys, the Grandiosemanipulative dimension predicted dominant interpersonal behavior and the Impulsive-irresponsible dimension predicted number of drug use disorder symptoms and frequency of alcohol use. Conversely, in girls, the Grandiose-manipulative dimension was most predictive of the number of drug use disorder symptoms, while the Impulsive-irresponsible was most predictive of dominant interpersonal behavior. Previous research in female adult samples has also indicated gender differences in the associations between psychopathic traits and external correlates such as anxiety (Salekin, Rogers, Sewell, 1997; Vitale \& Newman, 2001b; Vitale, Smith, Brinkley, \& Newman, 2002). In a similar vein, boys generally score significantly higher 
on adolescent psychopathy measures than girls (Andershed et al., 2007; Forth et al., 2003; YPI: Andershed et al., 2002). In all, these findings suggest that the manifestation of psychopathic traits in females differs from male manifestations.

\section{Methodological limitations}

Results from the present study should be qualified by several caveats. First, an examination of the nomological net surrounding adolescent psychopathy,should also include an examination of the convergence of different instruments measuring the same traits (Cronbach \& Meehl, 1955). In fact, previous studies have demonstrated that instruments such as the APSD, CPS, PCL:YV, and YPI are unique in their method and not isomorphic (Benning, Patrick, Salekin et al., 2005; Poythress et al., 2006; Skeem \& Cauffman, 2003). Future research should focus on the convergent validity of the Dutch YPI with other adolescent psychopathy measures. A recent study by Andershed et al., (2007) demonstrated moderate correlations between total scores and conceptually corresponding factor scores of the PCL:YV and the YPI.

A second limitation includes the sole reliance on self-report instruments in this study. A number of studies have identified self-report to be a valid assessment method for psychopathic traits in community youth (Andershed et al., 2002). However, an absence of information from other (professional) informants may have influenced the validity of ratings. Finally, exploratory factor analyses were performed to evaluate the structure of the YPI. As suggested above, future studies should perform confirmatory factor, and item response theory analysis in order to provide further information on the underlying structure of the YPI and the discriminating power of specific traits (e.g., Cooke \& Michie, 2001; Neumann et al., 2006).

Although a growing body of research has explored the nomological network of adolescent psychopathy, few studies have focused on psychopathic traits in non-referred adolescents from the perspective of general personality theory. In all, our findings strengthen current suggestions that the YPI is a 
time- and cost-effective instrument to assess and study psychopathic traits in community youth (Andershed et al., 2001; Andershed et al., 2002; Vaughn \& Howard, 2005). 


\section{$800^{2}$}

\section{$\because \cdots$}

$+\infty$ $-1-2+\cdots$

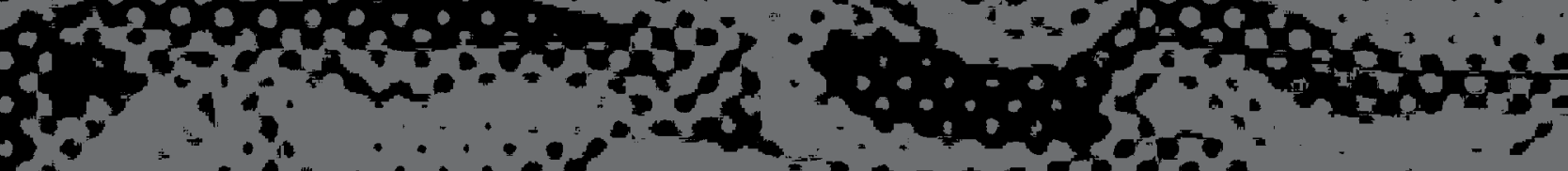

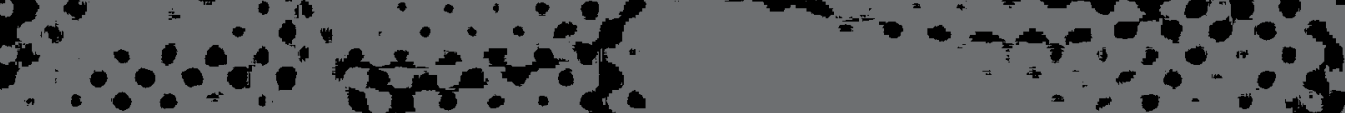

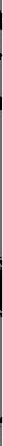
$\therefore \quad \cdots x:$ CHAPTE 7

$\because \quad 0$ General

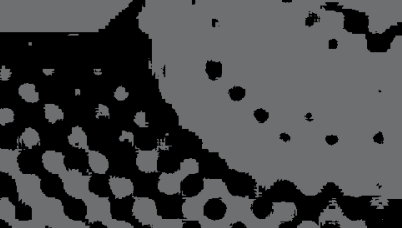

$\because \because \because \frac{1}{4}$
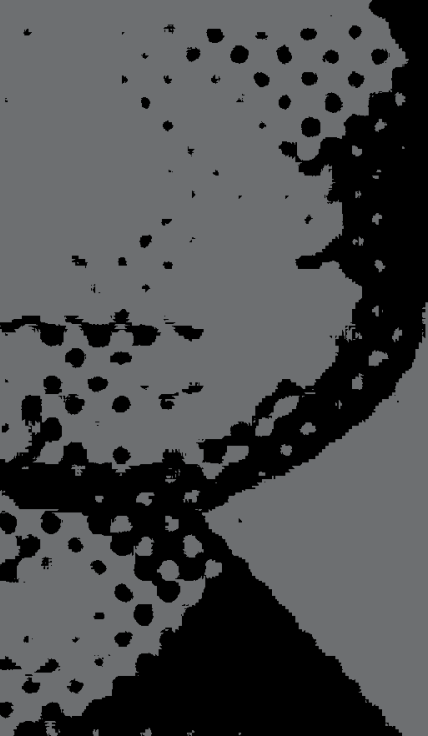

Diseussion $\because 8 \%$

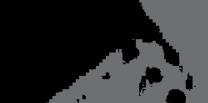
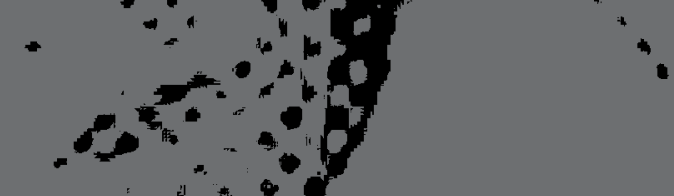

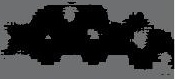

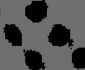

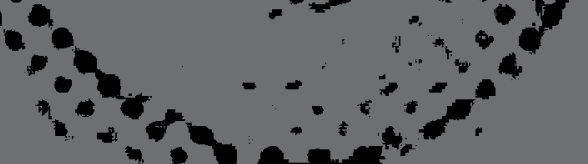

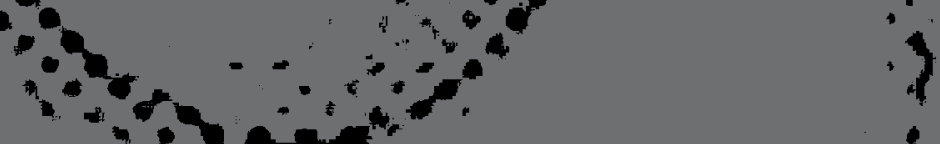


The interest of clinicians, researchers and policy makers in the PCL:YV is primarily related to its relevance for risk assessment in offender populations. Indeed, most studies of psychopathic traits in youth initially focused on their predictive power for (future) violence (Falkenbach, Poythress, \& Heide, 2003; Forth, Hart, \& Hare, 1990; Gretton, Hare, \& Catchpole, 2004; Gretton, McBride, O'Shaughnessy, \& Kumka, 2001). However, the PCL: $\mathrm{YV}$ is a diagnostic device by design and its role in forensic clinical practice goes well beyond risk assessment. The studies reported in this thesis were designed to examine the psychometric properties of the Dutch version of the Psychopathy Checklist: Youth Version, including its predictive validity. A secondary objective was to expand knowledge about the nomological network surrounding the construct of psychopathy and the psychopathy dimensions in youth.

\section{Main research findings}

The Dutch PCL:YV was found to be an internally consistent diagnostic measure that can be reliably scored by trained mental health professionals to measure the degree of psychopathic traits in boys and girls admitted to a juvenile justice treatment institution. These findings replicate international findings regarding the reliability of the PCL:YV (Brandt, Kennedy, Patrick, \& Curtin, 1997; Kosson, Cyterski, Steuerwald, Neumann, \& WalkerMatthews, 2002; O’Neill, Lidz, \& Heilbrun, 2003; Skeem \& Cauffman, 2003). Whereas the interrater reliablity for the total score of the PCL:YV was good, some of the instrument's items demonstrated an inadequate level of reliability, which calls for attention. Item Response Theory (IRT) analyses comparing the metric underlying adult and adolescent psychopathy scores, demonstrated an absence of Differential Test Functioning, indicating there is no age bias in the psychopathy total score as measured by the PCL:YV. However, low item-to-total correlations for some items (Impersonal sexual behavior, Early behavior problems, Lacks goals, Impulsivity and Serious violations of conditional release) in the female sample, suggested gender bias may be present. In particular, item descriptions of the behavioral items 
emphasize overt aggressive and antisocial behavior and may be appropriate for boys, but less suitable for girls, who tend to display more covert types of antisocial behavior, such as sexual promiscuity, prostitution, lying and manipulativeness (Odgers \& Moretti, 2002; Pepler, Madsen, Webster, \& Levene, 2005).

The construct validity of the Dutch PCL:YV in male adolescents was supported by meaningful relationships with a priori selected external criteria (see Chapter 2). Specifically, psychopathic traits in male adolescents were related to a dominant and hostile interpersonal style, antisocial and externalizing behaviors and substance abuse problems, and inversely associated with social introversion and a friendly and submissive interpersonal style. Similarly, exploratory analyses in the female sample produced theoretically meaningful associations between psychopathy scores and external correlates (see Chapter 3). Specifically, psychopathic traits were found to be related to assertiveness, competitiveness, tough-mindedness, selfconfidence, dominance, aggressiveness, behavioral problems, and the absence of physical complaints. However, a comparison of findings in both samples indicates differential patterns of associations. The most notable finding is that PCL:YV total scores in girls were not associated with any of the a priori selected MMPI-scales measuring antisocial (externalizing) behavior, substance abuse problems, and social introversion (except social avoidance). Similar to findings in adult female samples (for a review see, Nicholls \& Petrila, 2005), the present results identify psychopathy as a useful construct for understanding a small group of girls who demonstrate a large number of traits consistent with psychopathy. However, gender differences appear to play a role in the conceptualization and manifestation of psychopathic traits and, thereby contradict the assertion that the PCL:YV can be applied invariantly across gender as suggested in the PCL:YV manual (Forth, Kosson, \& Hare, 2003). Moreover, gender differences call for refinement of the psychopathy construct and its measurement for females.

In line with findings from a recent meta-analysis by Edens and Campbell (2007), psychopathic traits were found to be a significant and 
robust predictor of institutional disruptive behavior (physical violence in particular) in two samples of boys in juvenile justice treatment institutions. The present findings suggest that in samples where psychopathic traits are not so prevalent, the construct may only be useful for identifying adolescents at risk of physical violence but not for those at risk of other types of disruptive behavior. In samples where psychopathic traits are more prevalent, the presence of these traits is associated with various types of disruptive behavior, including verbal abuse and rule violation.

\section{Psychopathy dimensions}

In line with previous research on the PCL-R in adults (Cooke \& Michie, 2001) and the English language version of the PCL:YV in adolescents (Kosson et al., 2002), a three-factor model was found to underlie the Dutch PCL:YV. In this model, psychopathy is underpinned by three intercorrelated factors: an arrogant and deceitful interpersonal style (interpersonal dimension), a deficient affective experience (affective dimension), and an impulsive and irresponsible behavioral style (lifestyle dimension). From a model building perspective, the antisocial items were not found to be necessary features of psychopathy in the present research. In order to make any definitive decisions about the inclusion or exclusion of the antisocial items, future research should compare the structural validity of the three- and four-factor models in various populations (e.g., criminal-noncriminal; adult-adolescent; male-female), and their ability to predict aversive outcomes.

In the following section, the present findings regarding the psychometric properties of the separate psychopathy dimensions will be discussed. An important note is that these studies were limited to male adolescents. Considering the gender differences in psychopathy that were demonstrated in previous research (e.g., Odgers, Repucci, Moretti, 2005; Salekin, Rogers, \& Sewell, 1997; Schrum \& Salekin, 2006; Vitale \& Newman, 2001b; Vitale, Smith, Brinkley, \& Newman, 2002), and the research reported in Chapter 3 of this thesis, findings may not be generalizable to female adolescents. 


\section{Interpersonal dimension}

Adequate internal consistency and interrater reliability were demonstrated for the interpersonal dimension. In support of its construct validity, the interpersonal dimension was found to be related to a self-reported dominant interpersonal style, and inversely associated with social introversion. The IRT analyses identified the interpersonal items to generally demonstrate good discriminative power for distinguishing between adolescents exhibiting different levels of psychopathic traits. The presence of age bias was suggested by findings indicating that high scores on items 1 (Impression management), 2 (Grandiose sense of self worth), and 4 (Pathological lying) were less prevalent among adolescents than among adults. This finding may reflect raters' tendency to score these items conservatively in adolescents. However, there may also be a true difference in interpersonal style between adolescents and adults with psychopathic traits. Hypothetically, during adolescence, individuals with psychopathic traits may repeatedly experience interpersonal characteristics like manipulation and deceit as rewarding, thereby reinforcing this psychopathic interpersonal style.

The interpersonal dimension demonstrated little relevance for the prediction of institutional disruptive behavior. Nevertheless, it is still possible that the personality dimensions of psychopathy are related to more subtle indicators of treatment non-response, including program dropout and therapist ratings of non-participation. For instance, Hobson and Shine (2000) have demonstrated that Hare's traditional Factor 1 (and not Factor 2) was significantly associated with negative behavior during therapy groups and community meetings, and with general negative behavior on the ward.

\section{Affective dimension}

The affective dimension is generally considered to be the core dimension of psychopathy (Cooke \& Michie, 2001; Vincent, 2002). The present findings from IRT analyses in a sample of male adolescents support this notion, identifying this dimension as having the highest discriminative power and the least susceptibility to age influence. In addition, the affective dimension was 
found to have adequate internal consistency. However, the study described in Chapter 2 showed inadequate interrater reliability (single measure ICC $=.45$ ) for the affective dimension. In a meta-analysis by Campbell, Pulos, Hogan and Murrie (2005), interrater reliability was found to be better in samples with greater PCL:YV variability, whereas samples with a large number of subjects with uniformly low or high PCL:YV scores were more difficult for raters to agree upon. The authors argued that when the total or factor score variation decreases, reliability estimates decrease in magnitude because of range restriction. Indeed, in the seriously criminal Harreveld sample that was studied in Chapter 2, the four items of the affective factor were among those with the highest means while demonstrating limited variance. In contrast, in the less seriously criminal Rentray sample, greater variability in scores on the affective dimension may have influenced the outcome of a good interrater reliability (single measure ICC $=.71$ ). In this regard, it is important to note that IRT analyses identified the affective items to have limited discriminative power at high levels of the latent trait. The generally agreed upon notion that the affective dimension forms the core of psychopathy (Cooke \& Michie, 2001; Farrington, 2005; Vincent, 2002) calls for improved ways of measuring this dimension. Specifically, it may be necessary to extend the number of affective items to provide more information at higher levels of the latent trait.

Regarding construct validity, the affective dimension showed limited associations with external correlates in the studies reported here. In an extensive examination of the construct validity of the three Cooke and Michie (2001) factors in an adult sample, Hall, Benning and Patrick (2004) also found the affective factor to have few personality correlates. Hall et al. (2004) suggested that since psychopaths are characterized by a lack of insight regarding their own emotional deficits, it may be that the affective dimension is relatively resistant to full description via the self-report instruments that were used to measure external personality correlates. Future research may benefit from the inclusion of psychophysiological measures in the examination of construct validity since psychophysiological responses to 
emotional stimuli have been successfully used to identify emotional deficits in psychopathic adults (e.g., Patrick, Bradley, \& Lang, 1993), as well as in adolescents with psychopathic traits (Stevens, Charman, \& Blair, 2001)

\section{Lifestyle dimension}

The internal consistency and interrater reliability of the lifestyle dimension were good. Findings from IRT analyses indicated that age bias was not present for lifestyle items 3 (Stimulation seeking) and 15 (Irresponsibility), and the latter also demonstrated the strongest discriminative power. However, items 9 (Parasitic orientation) and 13 (Lacks goals) were more prevalent among adolescents than among adults. These items have been previously criticized for their lack of clear indications of the level at which this type of behavior becomes non-normative and indicative of psychopathy, thereby increasing the possibility of false positives (Seagrave \& Grisso, 2002). In support of its construct validity, the lifestyle dimension was found to be strongly related to impulsivity, authority problems, conduct problems, school problems and substance abuse problems. This dimension seems to tap a generalized tendency to engage in socially deviant behaviors, thereby overlapping substantially with the construct of externalizing behaviors (Hall et al., 2004). Finally, it is important to note that institutional physical violence was best predicted by the lifestyle dimension in the Rentray sample, whereas in the Harreveld sample it was best predicted by the antisocial dimension. This finding may perhaps be explained in light of the crossnational (Cooke, Hart and Michie, 2004), gender-related (Bolt, Vitale, Hare, \& Neumann, 2004; Schmidt et al., 2006) and age-related (Vincent, 2002) differences in the expression of psychopathy that have been demonstrated in previous research. Hypothetically, the lifestyle dimension is more characteristic of psychopathy in younger and (still) less criminal adolescents such as the Rentray sample, and is therefore more likely to be related to external correlates of psychopathy such as physical violence. In a similar vein, the antisocial dimension may be more revealing of psychopathy in older and more criminal adolescents such as the Harreveld sample. The lifestyle 
dimension may be an important precursor to antisocial behavior, which is expressed in the person's criminal lifestyle over time.

\section{Antisocial dimension}

The internal consistency of the antisocial dimension was good and the interrater reliability ranged from moderate to excellent in the various samples studied in this thesis. On the one hand, from a model building perspective, the antisocial items were demonstrated not to be necessary features in the assessment of psychopathic traits in male adolescent offenders. In addition, IRT analyses identified them as being the least discriminating and the most susceptible to age bias of all PCL:YV items. On the other hand, the lifestyle and antisocial dimensions were better predictors of institutional violence than either the interpersonal or the affective dimension. In addition, the antisocial dimension was the strongest predictor of institutional physical violence in a sample of seriously antisocial boys admitted to Jongerenhuis Harreveld. This finding is in line with studies demonstrating the four-factor model to be superior to the three-factor model in predicting violence (Hill, Neumann, \& Rogers, 2004; Vitacco, Neumann, \& Jackson, 2005). Moreover, the antisocial dimension was found to have incremental validity relative to the other three dimensions in predicting recidivism in six adult samples (Walters, Knight, Grann, \& Dahle, 2008). Finally, the antisocial dimension was most strongly associated with MMPIA clinical scale 4 (Psychopathic Deviate), measuring externalizing behaviors and antisocial personality characteristics. In all, these results suggest that from a theoretical perspective, the antisocial dimension should not be included in the assessment of psychopathy. However, associations with external correlates identify this dimension as having unique predictive utility as a marker of high risk antisocial behavior in youth. 


\section{Youth Psychopathic traits Inventory}

Evidence was provided for the psychometric properties of the Dutch version of the Youth Psychopathic Traits Inventory (YPI), an instrument for identifying psychopathic traits in the normal population. Exploratory Principal Components Analysis (PCA) identified a three-factor structure for the YPI that was highly similar to the model described by Andershed, Kerr, Stattin and Levander (2001). The model includes a Grandiose-manipulative, Callous-unemotional, and Impulsive-irresponsible dimension, conceptually resembling the interpersonal, affective and lifestyle dimensions of the PCL: YV. In the YPI, the three factors are broken down into 10 subdimensions (see Chapter 4). Boys were found to have significantly higher scores than girls on all subdimensions, except Impulsiveness. Reliability indices were good for the YPI total score, for the three factor scores and for all subdimensions except Unemotionality $(\alpha=.50)$ and Callousness $(\alpha=.32)$ in boys, and Callousness $(\alpha=.52)$ in girls. This is in line with previous studies in clinical samples which also found poor reliability indices for the Callousness subdimension (Poythress et al., 2006; Skeem \& Cauffman, 2003). These results suggest that the affective dimension is relatively resistant to reliable description via self-report (Hall et al., 2004), which is in line with similar findings on the affective dimension of the clinician-rated PCL:YV, as reported above.

Finally, in support of the construct validity of the YPI, psychopathic traits were related to a dominant and hostile interpersonal style, while inversely related to a submissive and friendly interpersonal style, both in boys and girls. Furthermore, psychopathic traits were associated with a higher frequency of self-reported drug and alcohol use. Also, some gender differences on the psychopathy dimensions were identified. Specifically, in boys, the Grandiose-manipulative dimension predicted dominant interpersonal behavior and the Impulsive-irresponsible dimension predicted number of drug use disorder symptoms and frequency of alcohol use. Conversely, in girls, the Grandiose-manipulative dimension was most predictive of the number of drug use disorder symptoms, while 
the Impulsive-irresponsible dimension was most predictive of dominant interpersonal behavior.

\section{Strengths of this thesis}

A prerequisite for professional and ethical use of the PCL:YV in clinical practice is a thorough examination of its psychometric properties. In line with a trend in the international literature (see Farrington, 2005), the present thesis submits the Dutch version of the PCL:YV to close psychometric scrutiny. Aspects of reliability and validity were examined to provide extensive information on how psychopathy scores could be appropriately interpreted in clinical practice. Another strength of the thesis is that the studies were conducted in forensic clinical settings, whereas most previous studies examining the psychometric properties of the PCL: YV were conducted in research settings (Edens \& Vincent, in press). In the present studies, the PCL:YV was employed in the way it is intended, i.e., by practicing clinicians in their daily work, thereby providing insight into "field reliability" of the PCL:YV. A final strength of this thesis is that the value of the psychopathy concept is evaluated in various samples (e.g., severely antisocial vs. less severely antisocial, boys vs. girls, forensic samples vs. normal population sample).

\section{Limitations}

Several limitations of the research presented in this thesis deserve attention. First, no prospective study into the predictive validity of the PCL:YV for criminal recidivism in the community was included. Although results were presented for disruptive behavior within the institution, the predictive validity of the PCL:YV for (violent) criminal recidivism after discharge from the institution, should be established in future research. A second limitation involves the inconclusiveness of the findings regarding the applicability of the PCL:YV to female adolescents. Our findings provide initial support for the reliability and validity of the PCL:YV for use in female adolescents. However, researchers should focus on methods which optimize reliability. A 
number of PCL:YV items seem of limited use to assess psychopathic traits in female adolescents. Furthermore, the nomological network surrounding psychopathy in female adolescents should be examined and compared to the nomological network surrounding psychopathy in male adolescents. Finally, the predictive validity for gender-specific aversive outcomes, such as academic difficulties (e.g., school drop out; Bates, Bayles, Bennett, Ridge, \& Brown, 1991), emotional problems (Zoccolillo, 1992), marital difficulties and violent relationships with men (Lewis et al., 1991; Robins, 1986), and poor parenting skills (Lewis et al., 1991) should be examined. In all, these types of studies would allow researchers to determine if and how gender-specific characteristics need to be considered in the assessment of psychopathic traits. In the meantime, clinicians should be cautious when using the PCL:YV with girls by synchronizing the clinical use of the PCL:YV with research developments. In addition, in line with previous suggestions by Nicholls and Petrila (2005) about the administration of the PCL-R to adult females, the administration of the PCL:YV should be accompanied by a careful consideration of a girl's background and psychological / psychiatric characteristics in order to determine the implications the psychopathic traits may have for the girl's functioning, level of risk and treatment. Third, differences in findings between the two forensic samples (Harreveld and Rentray) used in the study concerning predictive validity for institutional disruptive behavior, raises questions about the generalizability of findings to other populations (e.g., female adolescents, adolescents in detention programs). In addition, the finding that sexual offending was found to be a moderator of the relation between psychopathic traits and external correlates, suggests that there may also be limits to the generalizability of the present findings across offender types.

\section{Implications for clinical practice}

Based on the findings of this thesis and the practical experiences during the studies, a number of recommendations and some cautionary remarks for use of the PCL:YV in Dutch forensic clinical practice can be made. 


\section{The use of the PCL:YV}

Like any diagnostic instrument, the PCL:YV should only be used by trained mental health professionals. As instructed in the manual, raters should use a semi-structured interview as well as adequate collateral information. If the available collateral information is quantitatively or qualitatively insufficient, raters should first focus on retrieving extra information. In order to monitor reliability of individual professionals, a regular test of interrater reliability should accompany the use of the PCL:YV in clinical practice. In that way, rater drift can be identified and corrected in time.

The term psychopathy is generally considered to be pejorative since it is associated with an aura of dangerousness and untreatability. In fact, the use of a specific cut-off score to make a categorical distinction between psychopaths and non-psychopaths in youth is not supported by recent research findings (Murrie, Marcus, Douglas, Lee, Salekin, \& Vincent, 2007). As an alternative to labeling an adolescent as psychopathic, Farrington (2005) suggested to focus on the separate psychopathy dimensions.. However, in the present studies, the PCL:YV total score was found to be more reliable than dimension scores, and dimension scores were more reliable than item scores. Similarly, unequivocal evidence was provided for the construct validity and predictive validity of the PCL:YV total score, but findings regarding the psychopathy dimensions were more inconclusive. In addition, while age bias was found to be present for dimension scores, it did not affect the PCL: YV total score. Taken together, these findings suggest that the PCL:YV total score, and not the dimension scores, should serve as the basis for identifying psychopathic traits in adolescents. The use of the term 'psychopathic traits' seems warranted considering the large body of evidence regarding the clinical relevance of the identification of these traits. In order to avoid unjust use of the term, all professionals involved in the judicial system and the treatment of adolescent offenders have the responsibility to keep abreast of the state of the art in research into psychopathic traits in adolescents. Any stigmatizing effects can be further avoided by descriptions of the actual psychopathic symptoms that are observed in the individual case. Identifying these 
symptoms can assist clinical staff in planning treatment interventions and decision making regarding interpersonal attitude towards the youngster. For instance, an adolescent who is a frequent liar, will have to be confronted with this trait early in the treatment, and treatment staff will have to check on the adolescent's statements, especially at the beginning.

\section{Cautionary notes regarding the use of the PCL:YV in clinical practice}

The PCL:YV should not be used as a risk assessment instrument. For determining the level of risk of future (violent) criminal behavior, clinicians should apply instruments specifically designed for this purpose. For example, in the Structured Assessment of Violence Risk in Youth (SAVRY; Borum, Bartel, \& Forth; authorized Dutch translation: Lodewijks, Doreleijers, De Ruiter, \& De Wit-Grouls, 2001) historical, social, and individual risk factors are systematically evaluated and specific attention is directed at the possible influence of protective factors in reducing the risk of violence. However, empirical evidence clearly indicates that the presence of psychopathic traits predicts future antisocial behavior and therefore should play a crucial role in any comprehensive assessment of risk (Das, De Ruiter, Lodewijks, \& Doreleijers, 2007 [Chapter 5 of the present thesis]; Edens, Campbell, Weir, 2007; Falkenbach, Poythress, \& Heide, 2003; Forth et al., 1990; Gretton et al., 2004; Gretton et al., 2001). Whether the risk posed by psychopathic traits can be moderated by the presence of protective factors, remains an empirical question that awaits further study.

The presence of psychopathic traits is not a contra-indication for (offender) treatment, since an association between psychopathic traits and untreatability is not supported by empirical evidence. The fact that the current treatment literature suggests that nothing has worked in treating psychopathic individuals, does not mean nothing will work in the future (Wong \& Hare, 2005). Psychopathic traits should be targeted by treatment in order to reduce the risk of recidivism and efforts must be increased to explore possible effective treatment interventions. As outlined in the introduction, the What Works framework emphasizes the important role 
of the risk-need-responsivity principles and provides insightful ideas for improving treatment interventions for adolescents and adults with psychopathic traits.

\section{Suggestions for future research}

The results of the studies in the present thesis suggest several avenues for future research in Dutch adolescent forensic psychiatry. First of all, prospective research into the predictive validity of the Dutch PCL:YV for criminal recidivism after release is needed. This research should also evaluate whether any unique variance is explained by the PCL:YV once the predictive utility of other features is statistically controlled for. In this regard, it is interesting to note that psychopathy scores have been found to be equally predictive of general and violent recidivism as a more extensive risk assessment instrument, the Youth Level of Service / Case Management Inventory (YLS/CMI), suggesting “a lone personality construct can perform similarly to a more comprehensive risk assessment instrument" (p. 23, Edens et al., 2007). Second, the present findings regarding construct validity should be extended by measuring external criteria with methods other than self-report, including psychophysiological measures and observer reports. Third, the present findings regarding the association between psychopathic traits and institutional disruptive behavior should be extended with a more comprehensive set of variables chosen to measure the broader concept of treatment response. Fourth, a large body of international research (e.g., Frick \& Ellis, 1999; Lynam, 1996; Silverthorn \& Frick, 1999) demonstrated that psychopathic traits can be identified before adolescence and are highly prevalent in children with childhood-onset, life-course persistent conduct disorder. This type of research contributes to knowledge about the etiology of psychopathy. Recently, a child version of the YPI has been validated for the Dutch population (Van Baardewijk et al., in press). Future research should make use of this instrument to examine psychopathic traits in children from the general Dutch population. Finally, we suggest four topics of research that follow directly from this thesis: 1) improvement of the PCL:YV; 2) underlying 
structure of psychopathy 3) psychopathic traits in female adolescents; and 4) generalizability of findings. These four topics will be explained in the following paragraphs.

\section{Improvement of the PCL:YV}

Results from this and other studies suggest that the PCL:YV may have to be modified in order to optimize reliability of a number of its items. Similar to the strategy that was chosen in the development of the Comprehensive Assessment of Psychopathic Personality disorder (CAPP) for adults (Cooke, Hart, \& Logan, unpublished manuscript), an increase of the number of items that define the separate dimensions may result in more reliable measurement. In addition, it may be necessary to adjust the behavioral criteria of the PCL:YV to take into account gender-specific expressions of antisocial behavior. Furthermore, reliability may also be improved by using a multi-method approach (i.e., interviews, observations, psychophysiological assessment, file review) that has been advocated for the assessment of psychopathic traits in pre-adolescent children by Johnstone and Cooke (2004). Finally, more refined item descriptions which indicate clearly when the level of psychopathy symptoms becomes non-normative, may increase reliability at the item level (Salekin \& Frick, 2005).

\section{Underlying structure of psychopathy}

As stated before, any decisions about the inclusion or exclusion of antisocial items should be based on comparisons of the structural validity of the threeand four-factor models in various populations (e.g., criminal-noncriminal; adult-adolescent; male-female), and their ability to predict aversive outcomes. Furthermore, the interrelationships between the various psychopathy dimensions should be studied. Hare and Neumann (2005) suggested that the longitudinal relationships among psychopathy dimensions may be interactive and reciprocal in nature. For instance, rather than criminal behaviors being mere consequences of psychopathy, Knight and Sims-Knight (2003) found good fit for a model that hypothesized that physical and/or verbal abuse produced 
callousness. In addition, the present thesis found that while the lifestyle and antisocial dimensions most strongly predicted most forms of institutional disruptive behavior, correctional placement (which is only imposed after the most serious incidents) was best predicted by the interaction of all four dimensions. Similarly, previous research has demonstrated that the interaction of three PCL:YV dimensions (interpersonal, affective, and lifestyle) more strongly predicted future violent offending than any of the individual dimensions (Corrado, Vincent, Hart, \& Cohen, 2004; Vincent, Vitacco, Grisso, \& Corrado, 2003). These findings suggest that the interaction term may be more indicative of an overall psychopathy syndrome than the total score or any single dimension alone (Corrado et al., 2004). Or, put another way, it is possible that there are different types of psychopathy, depending on which of the dimensions predominates and on the interactive processes between dimensions (Walters et al., 2008). Thus, rather that considering psychopathy as a linear and additive construct (Psychopathy = affective + interpersonal + behavioral + antisocial), the construct should perhaps be viewed and studied from a nonlinear, interactive perspective, as shown in Figure 1.

FIGURE 1

THE RECIPROCAL AND INTERACTIVE RELATIONSHIPS DEFINING THE CONSTRUCT OF PSYCHOPATHY

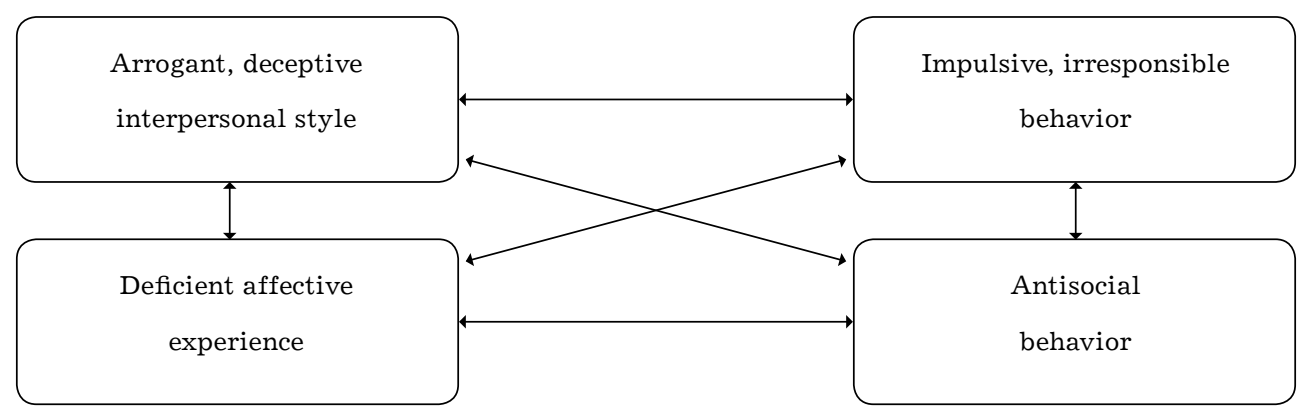




\section{Psychopathic traits in female adolescents}

In line with previous research (e.g., Odgers, Repucci, \& Moretti, 2005; Salekin et al., 1997; Vitale \& Newman, 2001b; Vitale, Smith, Brinkley, \& Newman, 2002), the present findings identify gender differences in psychopathic traits. It is time to take research into this subject 'to the next level'. For instance, a number of studies (e.g., Schrum \& Salekin, 2006) suggest that item response theory analyses (IRT) is a promising technique for identifying which PCL $\mathrm{R}$ or PCL:YV items are truly relevant for identifying psychopathic traits in females. In addition, prototypical analysis can be used as a bottom-up procedure for identifying gender-specific manifestations of psychopathic traits in females. In this type of analysis, clinicians are asked to identify the most typical or clear cases of a construct (Kotler \& McMahon, 2005). Finally, research in antisocial girls can be used to inform theoretical model building about the development and external correlates of psychopathic traits in girls. For example, Silverthorn and Frick (1999) proposed a delayed onset pathway in girls which is analogous to the childhood-onset pathway in boys. This delayed onset pathway in girls includes a callous and unemotional interpersonal style, that is thought to contribute to the development of antisocial behavior.

\section{Generalizability of findings}

Most research into psychopathic traits in adolescents has been performed in male adolescent offender samples. The generalizability to other populations has received little attention, thus far. First, in the present thesis, the juvenile justice samples comprised adolescents whose intellectual abilities were in the normal range. However, about half of the beds in juvenile justice treatment institutions in The Netherlands are occupied by intellectually disabled adolescents. In intellectually disabled adult offenders, the PCL-R was found to have adequate reliability and construct validity (Morrissey, Mooney, Hogue, Lindsay, \& Taylor, 2007) and to be significantly predictive of negative treatment progress in terms of a move to more restricted treatment conditions (Morrissey, Hogue et al., 2007). However, in contrast to what has 
been demonstrated in adult and adolescent offenders with normal intellectual abilities, psychopathy scores were not predictive of any type of institutional aggressive behavior (Morrissey et al., 2005). Future research should examine whether findings in this thesis are generalizable to the intellectually disabled adolescent offender population.

Second in the present thesis, some findings regarding construct validity differed between sex offenders versus non-sex offenders. Furthermore, previous findings in adult samples have indicated that 1) psychopathy scores differ across types of sexual offenders (e.g., rapists, child molesters, incest offenders; Barbaree, Seto, Serin, Amos, \& Preston, 1994; Olver \& Wong, 2006) and 2) there is an interaction between psychopathy factor scores and type of sexual offense (Porter et al., 2000). These findings suggest that future research should examine differences in the manifestation of psychopathic traits between different offender types.

Third, although the PCL:YV manual reports negligible associations between psychopathic traits and ethnicity in adolescents (Forth et al., 2003), concerns have been raised about the utility of psychopathy as a risk factor for future criminality among multiethnic offender samples (Edens \& Cahill, 2007). The juvenile justice samples that were studied in the present thesis comprised adolescents with different cultural backgrounds, thereby representing the ethnic composition of the total population of adolescent offenders in juvenile justice treatment institutions in The Netherlands in 2006 (www.dji.nl). However, the samples were too small to allow systematic comparisons between adolescents with different ethnic backgrounds. Future research should address the generalizability of the present findings across different ethnic backgrounds.

This thesis presents a small contribution to the solution of the fascinating puzzle called psychopathy. Recent research has documented neurocognitive deficits in children and adults suffering from this disorder (e.g., Blair, 1999; Blair \& Coles, 2000; Blair, Colledge, Murray, \& Mitchell, 2001). Recently, Blair, Peschardt, Budhani, Mitchell and Pine (2006) proposed a theoretical model, in which psychopathy is regarded as a 
neurodevelopmental disorder and a cognitive neuroscience perspective is used to explain psychopathy at the ultimate causal (genetic or social primary cause), molecular, neural, cognitive and behavioral level. This model provides an appealing framework for directing future research into the etiology of psychopathy. Hopefully, this type of research will help unravel the etiology and assist in the development of effective treatment strategies for a disorder that so often has a harmful course. 


\section{Background and goals of this thesis}

Psychopathy is a socially devastating disorder defined by a constellation of interpersonal (superficial charm, egocentricity, pathological lying, manipulativeness), affective (shallow emotions, lack of empathy, guilt, or remorse) and behavioral characteristics (impulsivity, irresponsibility, persistent violation of social norms and expectations; Cleckley, 1976; Hare, 1998; Wong \& Hare, 2005). The DSM-IV (American Psychiatric Association, 1994), requires a person to be 18 before a personality disorder may be diagnosed. However, like with any personality disorder, characteristics of psychopathy do not suddenly emerge in early adulthood. Psychopathic traits often become evident early in life and develop throughout childhood and adolescence (Forth \& Burke, 1998; Forth, Kosson, \& Hare, 2003). Early identification of these traits enhances the possibility of interventions early in life (Forth, Hart, \& Hare, 1990; Forth \& Mailloux, 2000; Frick, Bodin, \& Barry, 2000). At the same time, the negative connotations associated with the term psychopathy (e.g., dangerousness, untreatability) necessitate that high psychometric standards be set and met before psychopathy measures can be used in clinical forensic practice (Petrila \& Skeem, 2003; Skeem \& Cauffman, 2003).

For the assessment of psychopathic traits in adolescents aged 12 to 18 years, the Psychopathy Checklist: Youth Version (PCL:YV; Forth et al., 2003) is often used. The PCL:YV was adapted from the PCL-R (Hare, 1991, 2003), which is the most widely used measure of psychopathy assessment in adults. The PCL:YV is a 20 -item checklist which is completed by PCL trained professionals on the basis of a semi-structured interview and detailed collateral file information. Research has increasingly focused on the psychometric properties of the separate dimensions that are thought to underlie psychopathy (Cooke \& Michie, 2001). In contemporary international research, two models are compared in terms of structural validity, a three-factor model (Cooke \& Michie, 2001) and a four-factor model (Hare, 2003). Both models comprise an arrogant and deceitful interpersonal style (interpersonal dimension), deficient emotional experience (affective 
dimension), and an impulsive and irresponsible behavioral lifestyle (lifestyle dimension). The four-factor model includes an additional fourth factor comprising antisocial behaviors. At the moment, the Hare (2003) four-factor model is most attractive for research in terms of its comprehensiveness.

The studies reported in this thesis were designed to examine the psychometric properties of the Dutch version of the Psychopathy Checklist: Youth Version (Psychopathie Checklist: Jeugd Versie; PCL:JV; De Ruiter, Kuin, de Vries, \& Das, 2002), including its predictive validity. A second objective was to expand knowledge about the nomological network surrounding the construct of psychopathy and psychopathy dimensions in youth.

\section{Most important findings of this thesis}

The Dutch PCL:YV was found to be an internally consistent diagnostic measure that can be reliably scored by trained mental health professionals to measure the degree of psychopathic traits in adolescents in forensic clinical practice. The construct validity of the PCL:YV total score was supported by theoretically meaningful relationships with external criteria. The PCL:YV is a robust predictor of institutional disruptive behavior, in particular physical violence. Mixed findings are demonstrated regarding the reliability and construct validity of the four separate psychopathy dimensions. Although the affective dimension was found to be the most relevant dimension for the assessment of psychopathic traits in youth, its reliability and construct validity were inadequate. Results further indicate the presence of two types of biases in the assessment of psychopathic traits in adolescents, including gender differences in the manifestation of psychopathic traits and an age bias in scores on the interpersonal, lifestyle and antisocial dimensions (but not the PCL:YV total score). Finally, the present thesis provides support for the psychometric properties of the Youth Psychopathic traits Inventory (YPI; Andershed, Kerr, Stattin, \& Levander, 2002; Dutch authorized translation: Das \& De Ruiter, 2003) as a measure of psychopathic traits in adolescents in the general population. 


\section{SUMMARY OF THE CHAPTERS}

In Chapter 1, the various instruments that are available for the assessment of psychopathic traits in children and adolescents are presented. The contributions of research with these instruments in child and adolescent samples is discussed with respect to the construct validity of psychopathy, the factor structure underlying psychopathy, and the relationship between psychopathic traits and antisocial behavior. Next, the presence of gender differences in the manifestation of psychopathic traits is discussed, and an overview of research regarding the etiology and temporal stability of psychopathic traits is presented. Further, ethical and methodological issues pertaining to the diagnosis of psychopathy in youth are discussed. Finally, a description is provided of the setting in which the research reported in this thesis was conducted, and an outline of the main research questions is presented.

In Chapters 2 through 6, the empirical studies of this thesis are discussed. Chapter 2 concerns a study of the (interrater) reliability and construct validity of the Dutch PCL:YV in boys admitted to a juvenile justice treatment institution. Findings indicate that the PCL:YV as a whole is internally consistent and the interrater reliability by trained mental health professionals is adequate. In support of the construct validity, psychopathic traits were related to a dominant and hostile interpersonal style, to antisocial and externalizing behavior and to substance abuse problems, and inversely associated with social introversion and a friendly and submissive interpersonal style. The internal consistency of the four psychopathy dimensions was good. However, the affective dimension showed inadequate interrater reliability. The construct validity of the interpersonal, lifestyle and antisocial dimensions was supported by their relations to external correlates. For example, the interpersonal dimension was significantly associated with a self-reported dominant interpersonal style, while inversely associated with social introversion. Furthermore, the lifestyle dimension proved to be the strongest predictor of impulsivity, authority problems, 
conduct problems, school problems and substance abuse problems. Although the affective dimension was significantly associated with anger, associations with other external correlates (e.g., externalizing behavior, substance abuse, interpersonal style) were limited. Finally, different relationships for sexual offenders in comparison to non-sexual offenders were found between psychopathic traits and anxiety as well as conduct problems.

Chapter 3 presents the results of a study on the reliability and construct validity of the PCL:YV in a sample of female adolescents admitted to a secure juvenile justice treatment institution. Good internal consistency was demonstrated. However, low item-to-total correlations were obtained for some items (Impersonal sexual behavior, Early behavior problems, Lacks goals, Impulsivity and Serious violations of conditional release), which suggested the presence of a gender bias. Interrater reliability was found to be adequate at the level of dimension and total scores, but poor for some individual items. In support of the construct validity of the PCL:YV in girls, exploratory analyses demonstrated theoretically meaningful associations between psychopathic traits and external correlates. Specifically, psychopathic traits were found to be related to self-reported assertiveness, competitiveness, tough-mindedness, self-confidence, dominance, aggressiveness, behavioral problems, and an absence of physical complaints.

Chapter 4 describes a study on the structural and metric validity of the PCL:YV in a sample of boys from two juvenile justice treatment institutions and detailed file information of boys who were examined at the request of the court in order to determine criminal responsibility. Regarding the structural validity, support was provided for a modified version of the three-factor model proposed by Cooke and Michie (2001). In this model, psychopathy is underpinned by three intercorrelated factors: arrogant and deceitful interpersonal style (interpersonal dimension), deficient affective experience (affective dimension), and impulsive and irresponsible behavioral style (lifestyle dimension). Item Response Theory analysis was used to examine the metric validity of the PCL:YV by analyzing whether the separate items and/or the whole test operated differently for adolescents in 
comparison to adults. The affective items were identified as most relevant for assessing psychopathic traits in male adolescent offenders and were the least susceptible to age influence. Age influence was found to be present for the interpersonal, lifestyle, and antisocial dimensions. Specifically, adolescents were found to have lower scores on most interpersonal items but higher scores on most behavioral items than adults. Notable is that these effects disappeared at the complete test level, suggesting age bias is not present for the PCL:YV total score. Finally, the antisocial items and item 11 (Impersonal sexual behavior) and 17 (Unstable interpersonal relationships) were found to have the least relevance for the assessment of psychopathic traits in adolescents.

In Chapter 5, a prospective study on the predictive validity of the PCL:YV is presented. Psychopathic traits were demonstrated to be a significant predictor of institutional disruptive behavior (physical violence in particular) in a prospective study of two samples of boys in two different juvenile justice treatment institutions. The behavioral dimensions (lifestyle and antisocial), and not the personality dimensions (interpersonal and affective) of psychopathy, were found to be primarily responsible for the prediction of institutional problem behavior. Linear regression with the stepwise procedure identified that psychopathy (as defined by the interaction between all four dimensions) was more useful than the separate psychopathy dimensions in predicting the most serious incidents. Findings suggest that in samples where psychopathic traits are quite scarce, the construct may only be useful for identifying adolescents at risk of physical violence. In samples where psychopathic traits are more prevalent, the presence of these traits is associated with various types of disruptive behavior, including verbal abuse and rule violations.

The study in Chapter 6 provides support for the psychometric properties of the Dutch version of the YPI (YPI; Das \& De Ruiter, 2003) as an instrument for identifying psychopathic traits in the general Dutch adolescent population. Exploratory Principal Components Analysis (PCA) identified a three-factor structure, conceptually resembling the interpersonal, affective 
and lifestyle dimensions of the PCL:YV. The three factors are broken down into ten subdimensions. Boys were found to have significantly higher scores than girls on all subdimensions, except Impulsiveness. Reliability indices were good for the YPI total score, for the three factor scores and for all subdimensions, except Unemotionality $(\alpha=.50)$ and Callousness $(\alpha=.32)$ in boys, and Callousness $(\alpha=.52)$ in girls. Finally, in support of the construct validity of the YPI, psychopathic traits were significantly and positively related to a dominant and hostile interpersonal style, while significantly inversely related to a submissive and friendly interpersonal style.

Furthermore, psychopathic traits were associated with a higher frequency of self-reported drug and alcohol use.

Chapter 7 summarizes the most important findings of the studies presented in this thesis. Furthermore, strengths and limitations of the studies presented in this thesis are discussed. Next, implications for clinical practice are described. For example, it is suggested that the use of the PCL: $\mathrm{YV}$ in clinical practice should be accompanied by a regular test of interrater reliability in order to monitor reliability of individual professionals. In addition, it is suggested that all professionals involved in the judicial system and the treatment of adolescent offenders have the responsibility to keep abreast of the scientific developments in the assessment of psychopathic traits in adolescents. This chapter is concluded with suggestions for future research regarding the improvement of the PCL:YV, the factor-structure underlying the PCL:YV and the generalizability of the present findings. 


\section{Achtergrond en doelstelling van dit proefschrift}

Psychopathie is een stoornis met zeer ernstige sociaal-maatschappelijke gevolgen, en wordt gekenmerkt door interpersoonlijke (oppervlakkige charme, egocentriciteit, pathologisch liegen, manipulatie), affectieve (oppervlakkige emoties, gebrek aan empathie, schuldgevoel of berouw) en gedragsmatige kenmerken (impulsiviteit, onverantwoordelijk gedrag, aanhoudende schending van sociale normen en verwachtingen; Cleckley, 1976; Hare, 1998; Wong \& Hare, 2005). Volgens de DSM-IV (American Psychiatric Association, 1994) mag pas vanaf de leeftijd van 18 jaar een persoonlijkheidsstoornis gediagnosticeerd worden. Echter, zoals bij elke persoonlijkheidsstoornis, ontstaan kenmerken van psychopathie niet opeens in de jongvolwassenheid. Psychopathische trekken manifesteren en ontwikkelen zich al vroeg in de kindertijd en adolescentie (Forth \& Burke, 1998; Forth, Kosson, \& Hare, 2003). Dergelijke trekken dienen zo vroeg mogelijk geïdentificeerd te worden, want alleen dan is vroegtijdige interventie mogelijk (Forth, Hart, \& Hare, 1990; Forth \& Mailloux, 2000; Frick, Bodin, \& Barry, 2000). Tegelijkertijd vereisen de negatieve connotaties die geassocieerd zijn met een diagnose psychopathie (zoals gevaarlijkheid, onbehandelbaarheid) dat diagnostische instrumenten voor de forensische praktijk aan hoge psychometrische standaarden voldoen (Petrila \& Skeem, 2003; Skeem \& Cauffman, 2003).

Voor de diagnostiek van psychopathische trekken bij adolescenten van 12 tot 18 jaar, wordt internationaal voornamelijk de Psychopathy Checklist: Youth Version (PCL:YV; Forth et al., 2003) gebruikt. De PCL:YV is afgeleid van de PCL-R (Hare, 1991, 2003), het meest gebruikte instrument voor het diagnosticeren van psychopathie bij volwassenen. De PCL:YV is een checklist bestaande uit 20 items en wordt door in de PCL getrainde professionals gescoord op basis van een semi-gestructureerd interview en gedetailleerde collaterale informatie. De laatste jaren richt het wetenschappelijk onderzoek naar psychopathie zich meer en meer op de psychometrische eigenschappen van de afzonderlijke dimensies van psychopathie (Cooke \& Michie, 2001). In de huidige internationale vakliteratuur wordt de structurele validiteit van 
twee modellen met elkaar vergeleken; het drie-factor model (Cooke \& Michie, 2001) versus het vier-factor model (Hare, 2003). Beide modellen omvatten een arrogante en leugenachtige interpersoonlijke stijl (interpersoonlijke dimensie), kilheid en gebrek aan empathie (affectieve dimensie), en een levensstijl gekenmerkt door impulsief en onverantwoordelijk gedrag (levensstijl dimensie). Het vier-factor model heeft daarenboven nog een vierde factor die antisociale gedragingen omvat. Gezien zijn volledigheid, lijkt het vier-factor model het meest aantrekkelijk voor gebruik in onderzoek.

De onderzoeken die beschreven worden in dit proefschrift zijn ontworpen om de psychometrische eigenschappen van de Nederlandstalige versie van de Psychopathy Checklist: Youth Version (Psychopathie Checklist: Jeugd Versie; PCL:JV; De Ruiter, Kuin, de Vries, \& Das, 2002) te onderzoeken. Meer specifiek gaat het om de betrouwbaarheid, de constructvaliditeit en de predictieve validiteit van het instrument. Een bijkomende doelstelling van het onderzoek is om de kennis over het nomologische netwerk rondom psychopathie en de afzonderlijke psychopathiedimensies bij jongeren te verkennen. Middels het nomologisch netwerk worden relaties tussen theoretische constructen onderling, tussen theoretische constructen en hun operationalisaties en tussen operationalisaties onderling vastgelegd en kan bepaald worden of het construct (in dit geval psychopathie en de afzonderlijke psychopathiedimensies) bestaansrecht heeft.

\section{De belangrijkste bevindingen van dit proefschrift}

De studies in dit proefschrift tonen aan dat de PCL:YV goede psychometrische eigenschappen heeft voor het meten van psychopathische trekken bij jeugdigen in de Nederlandse forensische jeugdpsychiatrie, waaronder een goede interne consistentie en interbeoordelaarsbetrouwbaarheid. De construct validiteit van de PCL: YV wordt ondersteund door theoretisch betekenisvolle relaties tussen de PCL:YV totaalscore en externe variabelen. Verder wijzen de resultaten uit dat de PCL:YV score een sterke voorspeller is van grensoverschrijdend 
gedrag in de inrichting, in het bijzonder van fysiek geweld. Met betrekking tot de betrouwbaarheid en constructvaliditeit van de afzonderlijke psychopathiedimensies zijn de bevindingen wat minder eenduidig. Zo blijkt de affectieve dimensie de belangrijkste dimensie voor het meten van psychopathische trekken bij jongeren te zijn, terwijl er tegelijkertijd sprake is van een lage interbeoordelaarsbetrouwbaarheid en constructvaliditeit.

In dit proefschrift is onderzocht in hoeverre sekse en leeftijd van invloed zijn op PCL:YV scores. Uit de resultaten blijkt dat de gedragsmatige items nauwelijks bijdragen aan de psychopathie totaalscore bij meisjes maar juist wel bij jongens. Daarnaast zijn er verschillen tussen jongens en meisjes in de relaties tussen de PCL:YV en externe variabelen die conceptueel gerelateerd zijn aan psychopathische trekken, waaronder externaliserend gedrag, middelenmisbruik, impulsiviteit en een dominante en vijandige interpersoonlijke stijl. Deze bevindingen duiden op sekseverschillen in de manifestatie van psychopathische trekken bij adolescenten. Verder wijzen de resultaten uit dat hoewel leeftijd geen invloed heeft op de totaalscore van de PCL:YV, dit wel blijkt te gelden voor de interpersoonlijke en de antisociale psychopathiedimensies.

Tenslotte blijkt uit het onderzoek dat de Youth Psychopathic traits Inventory (YPI; Andershed, Kerr, Stattin, \& Levander, 2002; geautoriseerde Nederlandse vertaling: Das \& De Ruiter, 2003) goede psychometrische eigenschappen bezit en gebruikt kan worden als een instrument voor het vaststellen van psychopathische trekken bij adolescenten in de normale populatie. 


\section{SAMENVATTING VAN DE HOOFDSTUKKEN}

In Hoofdstuk 1 worden de verschillende instrumenten voor het meten van psychopathische trekken bij kinderen en adolescenten besproken. Resultaten uit onderzoek naar de constructvaliditeit van psychopathische trekken, de onderliggende factorstructuur en de relatie tussen psychopathische trekken en antisociaal gedrag, worden uiteengezet. Vervolgens wordt de mogelijke aanwezigheid van sekseverschillen in de wijze waarop psychopathische trekken zich manifesteren besproken en wordt een overzicht gegeven van onderzoek naar de etiologie en de temporele stabiliteit van psychopathische trekken. Daarna volgt een bespreking van de ethische en methodologische dilemma's die samenhangen met het gebruik van de diagnose psychopathie bij jeugdigen. Tenslotte wordt de setting waarin de huidige onderzoeken zijn uitgevoerd besproken en worden de centrale onderzoeksvragen gepresenteerd.

In de hoofdstukken 2 tot en met 6 worden de empirische onderzoeken van deze dissertatie besproken. In Hoofdstuk 2 staan de (interbeoordelaars)betrouwbaarheid en constructvaliditeit van de Nederlandstalige PCL:YV voor het meten van psychopathische trekken bij jongens in een gesloten justitiële behandelinrichting centraal. De interne consistentie van de PCL: YV bleek goed te zijn en het instrument kan door getrainde professionals uit de forensische psychiatrie betrouwbaar gecodeerd worden. De constructvaliditeit was goed: psychopathische trekken bleken gerelateerd te zijn aan een dominante en vijandige interpersoonlijke stijl, antisociaal en externaliserend gedrag en problemen gerelateerd aan middelenmisbruik. Er bestond een omgekeerde relatie tussen psychopathische trekken enerzijds, en sociale introversie en een vriendelijke en onderdanige interpersoonlijke stijl, anderzijds. De interne consistentie van de vier psychopathiedimensies was goed. Echter, de interbeoordelaarsbetrouwbaarheid van de affectieve psychopathiedimensie was onvoldoende. De constructvaliditeit van de interpersoonlijke, de impulsieve levensstijl en de antisociale dimensies werd ondersteund door hun betekenisvolle relaties met externe variabelen. Zo bleek de interpersoonlijke dimensie bijvoorbeeld met name gerelateerd 
te zijn aan een zelfgerapporteerde dominante interpersoonlijke stijl en omgekeerd gerelateerd te zijn aan sociale introversie. De levensstijl dimensie daarentegen, bleek juist sterk gerelateerd te zijn aan impulsiviteit, autoriteitsproblemen, gedragsproblemen, en problemen met school en drugs. Hoewel de affectieve dimensie geassocieerd was met zelfgerapporteerde woede, waren de relaties met andere externe variabelen beperkt. Tenslotte bleek de relatie tussen psychopathische trekken en zelfgerapporteerde angst en gedragsproblemen anders te zijn voor seksuele delinquenten in vergelijking met niet-seksuele delinquenten.

Hoofdstuk 3 beschrijft de resultaten van een onderzoek naar de betrouwbaarheid en constructvaliditeit van de PCL:YV in een steekproef van vrouwelijke adolescenten in een gesloten justitiële behandelinrichting. Er is sprake van een goede interne consistentie van de PCL:YV. Echter, de lage item-totaal correlaties voor sommige items (Onpersoonlijk seksueel gedrag, Gedragsproblemen op jonge leeftijd, Gebrek aan doelen op de lange termijn, Impulsiviteit en Ernstig schending van voorwaarden) suggereert dat deze items minder geschikt zijn voor toepassing bij vrouwelijke proefpersonen. De interbeoordelaarsbetrouwbaarheid bleek goed te zijn op het niveau van dimensie- en totaalscores, maar was zwak voor de individuele items van de lijst. Psychopathische trekken bleken theoretisch betekenisvolle relaties te vertonen met externe variabelen. Zo bleek dat psychopathische trekken gerelateerd waren aan zelfgerapporteerde assertiviteit, een stoere houding, zelfverzekerdheid, dominantie, agressiviteit, gedragsproblemen, en de afwezigheid van somatische klachten. Deze bevindingen ondersteunen de constructvaliditeit van de PCL:YV bij vrouwelijke adolescenten.

In het onderzoek dat beschreven wordt in Hoofdstuk 4 wordt de structurele en metrische validiteit van de PCL:YV onderzocht in een gecombineerde steekproef van jongens uit twee verschillende justitiële jeugdinrichtingen en dossiers van jongens die een Pro Justitia persoonlijkheidsonderzoek hadden ondergaan. Wat betreft de structurele validiteit, bleek een aangepaste versie van het drie-factor model ten grondslag te liggen aan psychopathie bij mannelijke adolescenten. 
Dit model omvat een arrogante en leugenachtige interpersoonlijke stijl (interpersoonlijke dimensie), kilheid en gebrek aan empathie (affectieve dimensie), en een levensstijl gekenmerkt door impulsief en onverantwoordelijk gedrag (levensstijl dimensie). Met behulp van Item Response Theory analyses zijn twee aspecten van metrische validiteit onderzocht: de relevantie van de PCL:YV items voor het meten van psychopathische trekken bij adolescenten en mogelijke verschillen tussen adolescenten en volwassenen in het functioneren van zowel de afzonderlijke items als de totaalscore. De affectieve items bleken het meest relevant te zijn voor het meten van psychopathische trekken, daarnaast had leeftijd de geringste invloed op de scoring van deze items. Leeftijd bleek wel van invloed te zijn op de scores op de interpersoonlijke, levensstijl en antisociale dimensies. Zo blijken adolescenten in vergelijking met volwassenen structureel lager te scoren op de interpersoonlijke items en structureel hoger te scoren op de meeste gedragsmatige items. Opvallend is dat deze effecten verdwenen als het hele instrument in de analyse werd opgenomen, hetgeen erop duidt dat het scoren van de gehele PCL:YV niet beïnvloed wordt door leeftijd. Tenslotte wezen de resultaten uit dat de antisociale items en de items 11 (Onpersoonlijk seksueel gedrag) en 17 (Instabiele interpersoonlijke relaties) de minste relevantie hebben voor het meten van psychopathische trekken bij adolescenten.

In Hoofdstuk 5 wordt een prospectief onderzoek beschreven naar de predictieve validiteit van de PCL:YV. Psychopathische trekken bleken in twee steekproeven van jongens in twee verschillende justitiële behandelinrichtingen een significante voorspeller te zijn van problematisch gedrag in de inrichting, met name fysiek gewelddadig gedrag. De gedragsdimensies (levensstijl en antisociaal), en niet de persoonlijkheidsdimensies, bleken verantwoordelijk te zijn voor de voorspelling van storend gedrag in de inrichting. Met behulp van lineaire regressie-analyse werd onderzocht of de PCL:YV totaalscore (zoals gemeten aan de hand van het interactie-effect van de vier psychopathie dimensies) iets toevoegde aan de voorspellende waarde van de afzonderlijke dimensies. 
De resultaten wezen uit dat de psychopathie totaalscore de meest krachtige voorspeller is voor ernstige incidenten die een correctieplaatsing of plaatsing in de afzondering tot gevolg hebben. Uit een vergelijking van de bevindingen tussen beide steekproeven blijkt dat in een steekproef waar psychopathische trekken niet vaak voorkomen, deze trekken alleen van waarde zijn voor de voorspelling van fysiek geweld. Als psychopathische trekken wél frequent voorkomen, is hun aanwezigheid voorspellend voor meerdere typen grensoverschrijdend gedrag in de inrichting, waaronder verbaal geweld en regelovertredingen.

Uit het onderzoek dat beschreven wordt in Hoofdstuk 6 blijkt dat de Nederlandse versie van de Youth Psychopathic traits Inventory goede psychometrische eigenschappen heeft en gebruikt kan worden als een instrument voor het identificeren van psychopathische trekken bij adolescenten in de normale populatie. Met behulp van exploratieve Principale Componenten Analyse werd een drie-factor structuur geïdentificeerd die conceptueel overeenkomt met de interpersoonlijke, affectieve en levensstijldimensies van de PCL:YV. De drie factoren worden onderverdeeld in tien subdimensies. Jongens bleken op al deze subdimensies, behalve op de subdimensie Impulsiviteit, hoger te scoren dan meisjes. De interne consistentie bleek goed te zijn voor de YPI totaalscore, voor de drie factoren en voor alle subdimensies behalve Affectieve vlakheid $(\alpha=.50)$ en Kilheid ( $\alpha=.32$ ) bij jongens, en Kilheid bij meisjes $(\alpha=.52)$. Tenslotte werd de constructvaliditeit van de YPI ondersteund door de significante, positieve relatie tussen psychopathische trekken en een dominante en vijandige interpersoonlijke stijl, en door de inverse relatie met een onderdanige en vriendelijke interpersoonlijke stijl. Verder bleken psychopathische trekken gerelateerd te zijn aan een hogere frequentie van zelfgerapporteerd drugs- en alcoholgebruik.

In Hoofdstuk 7 wordt de balans van dit proefschrift opgemaakt. De belangrijkste bevindingen worden samengevat en de sterke punten en de beperkingen van de onderzoeken worden geïnventariseerd. Daarna worden de implicaties voor het gebruik van de PCL:YV in de 
klinische praktijk besproken. Zo wordt geadviseerd om met regelmaat de interbeoordelaarsbetrouwbaarheid van psychopathiescores te bepalen om te voorkomen dat de scores van afzonderlijke beoordelaars in de loop der tijd steeds verder van elkaar gaan afwijken. Daarnaast wordt gesteld dat alle professionals die in de forensische jeugdsector werkzaam zijn, de verantwoordelijkheid hebben om op de hoogte te zijn en te blijven van de stand van zaken binnen het onderzoek naar psychopathische trekken bij jeugdigen. Het hoofdstuk wordt afgesloten met suggesties voor toekomstig onderzoek naar psychopathische trekken bij jeugdigen en de onderliggende factorstructuur van de PCL:YV , met betrekking tot mogelijke verbeteringen van de PCL:YV, en de generaliseerbaarheid van de huidige bevindingen. 
Achenbach, T.M. (1991). Integrative

Guide for the 1991 CBCL4-18, YSR and TRF Profiles. Burlington: University of Vermont, Department of Psychiatry.

Algemene Rekenkamer (2007). Detentie, behandeling en nazorg criminele jeugdigen. [Detention, treatment, and aftercare for young offenders]. 's Gravenhage: Sdu Uitgevers.

American Psychiatric Association (1994). Diagnostic and statistical manual of mental disorders ( $4^{\text {th }}$ ed.). Washington, DC: Author.

Andershed, H, Kerr, M., Stattin, H., \& Levander, S. (2001). Psychopathic traits in non-referred youths: Initial test of a new assessment tool. In E. Blaauw, \& L. Sheridan (Eds.), Psychopaths: Current international perspectives (pp. 131-158). Den Haag: Elsevier.

Andershed, H., Gustafson, S.B., Kerr, M., Stattin, H. (2002). The usefulness of selfreported psychopathy-like traits in the study of antisocial behavior among nonreferred adolescents. European Journal of Personality, 16, 383-402.

Andershed, H., Hodgins, S., \& Tengström, A. (2007). Convergent validity of the
Youth Psychopathic Traits Inventory (YPI): Association with the Psychopathy Checklist: Youth Version (PCL:YV). Assessment, 14, 144-154.

Andershed, H., Kerr, M., Stattin, H., \& Levander, S. (2001). Psychopathic traits in non- referred youths: Initial test of a new assessment tool. In E. Blaauw, \& L. Sheridan (Eds.), Psychopaths: Current international perspectives (pp. 131-158). Den Haag: Elsevier. Andrews, D.A. (1995). The psychology of criminal conduct and effective treatment. In: J. McGuire (ed.) What works: Reducing reoffending, guidelines from research and practice. Chichester: Wiley, 35-62.

Andrews, D.A., \& Bonta, J. (2003). The psychology of criminal conduct ( $3^{\text {rd }}$ ed.). Cincinnati: OH: Anderson Press.

Andrews, D.A., Bonta, J., \& Hoge, R.D. (1990). Classification for effective rehabilitation. Rediscovering psychology. Criminal Justice and Behavior, 17, 19-52. Andrews, D.A., \& Dowden, C. (2006). Risk principle of case classification in correctional treatment. A meta-analytic investigation. International Journal of Offender Therapy and Comparative Criminology, 50, 88-100. 
Baardewijk, Y., van, Stegge, H.,

Andershed, H., Thomaes, S., Scholte, E., \& Vermeiren, R. (in press). Measuring psychopathic traits in children through self-report. The development of the Youth Psychopathic traits Inventory - Child Version. International Journal of Law and Psychiatry.

Barbaree, H.E., Seto, M.C., Serin, R., Amos, N., \& Preston, D. (1994). Comparisons between sexual and nonsexual rapist subtypes: Sexual arousal to rape, offense precursors, and offense characteristics. Criminal Justice and Behavior, 21, 95-114.

Bates, J.E., Bayles, K., Bennett, D.S., Ridge, B., \& Brown, M.M. (1991). Origins of externalizing behavior: Problems at eight years of age. In D. J. Pepler \& K. H. Rubin (Eds.), The development and treatment of childhood aggression (p.p. 93-120). Hillsdale, NJ: Erlbaum

Belfrage, H., Fransson, G., \& Strand, S. (2000). Prediction of violence using the HCR-20 : A prospective study in two maximum-security correctional institutions. Journal of Forensic Psychiatry, 11, 167-175.
Benning, S.D., Patrick, C.J., Blonigen, D.M., Hicks, B.M., \& Iacono, W.G. (2005). Estimating facets of psychopathy from normal personality traits: A step toward community-epidemiological investigations. Assessment, 12, 3-18.

Benning, S.D., Patrick, C.J., Salekin, R.T., \& Leistico, A.R. (2005). Convergent and discriminant validity of psychopathy factors assessed via self-report: A comparison of three instruments. Assessment, 12, 270-289.

Berman, A.H., Bergman, H., Palmstierna, T., \& Schlyter, F. (2002). Evaluation of the Drug Use Disorders Identification Test (DUDIT) in criminal justice and detoxification settings and in a Swedish population sample. European Addiction Research, 11, 22-31.

Blair, R.J.R. (1995). A cognitive developmental approach to morality: Investigating the psychopath. Cognition, 57, 1-29.

Blair, R. J. R. (1999). Responsiveness to distress cues in the child with psychopathic tendencies. Personality and Individual Differences, 27, 135-145. 
Blair, R.J.R. (2004). The roles of orbital

frontal cortex in the modulation

of antisocial behaviour. Brain and

Cognition, 55, 198-208.

Blair, R.J.R. (2005). Applying a cognitive neuroscience perspective to the disorder of psychopathy. Development and

Psychopathology, 17, 865-891.

Blair, R. J. R., \& Coles, M. (2000).

Expression recognition and behavioral problems in early adolescence. Cognitive Development, 15, 421-434.

Blair, R. J. R., Colledge, E., Murray, L., \& Mitchell, D. G. V. (2001). A selective impairment in the processing of sad and fearful expressions in children with psychopathic tendencies. Journal of Abnormal Child Psychology, 29, 491-498.

Blair, R.J.R., Monson, J., \& Frederickson, N. (2001). Moral reasoning and conduct problems in children with emotional and behavioural difficulties. Personality and Individual Differences, 31, 799-811.

Blair, R.J.R., Peschardt, K.S., Budhani, S., Mitchell, D.G.V., \& Pine, D. S. (2006). The development of psychopathy. Journal of Child Psychology and Psychiatry, 47, 262-275.
Blonigen, D.M., Carlson, S.R., Krueger, R.F., \& Patrick, C.J. (2003). A twin study of self-reported psychopathic personality traits. Personality and Individual Differences, 35, 179-197.

Blonigen, D.M., Hicks, B.M., Krueger, R.F., Patrick, C.J., \& Iacono, W.G. (2005). Psychopathic personality traits: Heritability and genetic overlap with internalizing and externalizing psychopathology. Psychological Medicine, 35, 637-648.

Blonigen, D.M., Hicks, B.M., Krueger, R.F., Patrick, C.J., \& Iacono, W.G. (2006). Continuity and change in psychopathic traits as measured via normal-range personality: A longitudinal-biometric study. Journal of Abnormal Psychology, 115, 85-95.

Boer, D.P., Hart, S.D., Kropp, P.R., \& Webster, C.D. (1997). Manual for the Sexual Violence Risk-20. Professional guidelines for assessing risk of sexual violence. Vancouver, BC: British Columbia Institute against Family Violence.

Bolt, D.M., Hare, R.D., Vitale, J.E., \& Newman, J.P. (2004). A multigroup item response theory analysis of 
the Psychopathy Checklist-Revised.

Psychological Assessment, 16, 155-168.

Borum, R., Bartel, P. \& Forth, A.: Manual

for the Structured Assessment of Violence

Risk in Youth (SAVRY). University of

South Florida, 2003.

Brandt, J.R., Kennedy, W.A., Patrick, C.J., \& Curtin, J. (1997). Assessment of psychopathy in a population of incarcerated adolescent offenders. Psychological Assessment, 9, 429-435.

Bullens, R. \& Hendriks, J. (1999).

Seksualiteits Vragenlijst Pakket voor

Adolescenten. [Sexuality Assessment

Package for Adolescents]. Unpublished research version.

Butcher, J.N., Williams, C.L., Graham, J.R., Archer, R.P., Tellegen, A., Ben-

Porath, Y.S., et al. (1992). MMPI-A

(Minnesota Multiphasic Personality

Inventory-Adolescent): Manual

for administration, scoring, and interpretation. Minneapolis: University of Minnesota Press.

Butcher, J.N., Dahlstrom, W.G., Graham, J.R., Tellegen, A., \& Kaemmer, B. (1989). Minnesota Multiphasic Personality Inventory-2 (MMPI-2): Manual for
Administration and scoring. Minneapolis: University of Minnesota Press.

Caldwell, M., Skeem, J., Salekin, R., \& Van Rybroek, G. (2006). Treatment response of adolescent offenders with psychopathy features: A 2-year follow-up. Criminal Justice and Behavior, 33, 571596.

Cale, E.M., \& Lilienfeld, S.O. (2002). Histrionic personality disorder and antisocial personality disorder: Sexdifferentiated manifestations of psychopathy? Journal of Personality Disorders, 16, 52-72.

Campbell, J.S., Pulos, S., Hogan, M., Murry, F. (2005). Reliability generalization of the Psychopathy Checklist applied in youthful samples. Educational and Psychological Measurement, 65, 640-656.

Caputo, A.A., Frick, P.J., \& Brodsky, S.L. (1999). Family violence and juvenile sex offending: The potential mediating role of psychopathic traits and negative attitudes toward women. Criminal Justice and Behavior, 26, 338-356.

Caspi, A., Block, J., Block, J.H., Klopp, B., Lynam, D., Moffit, E., \& StouthamerLoeber, M. (1992). A common-language 
version of the California Child Q-set for personality assessment. Psychological Assessment, 4, 512-523.

Christian, R.E., Frick, P.J., Hill, N.L., Tyler, L., \& Frazer, D.R. (1997). Psychopathy and conduct problems in children: Implications for subtyping children with conduct problems. Journal of the American Academy of Child and Adolescent Psychiatry, 36, 233-241.

Cleckley, H. (1976). The mask of sanity ( $5^{\text {th }}$ ed.). St. Louis, MO: Mosby.

Cooke, D.J., Hart, R.D., \& Logan, C. (2004). Comprehensive Assessment of Psychopathic Personality DisorderInstitutional Rating Scale. Unpublished manuscript.

Cooke, D.J., Hart, R.D., \& Michie, C. (2004). Cross-national differences in the assessment of psychopathy: Do they reflect variations in raters' perceptions of symptoms? Psychological Assessment, $16,1-5$.

Cooke, D.J., Kosson, D., \& Michie, C. (2001). Psychopathy and ethnicity: Structural, item, and test generalizability of the Psychopathy Checklist-Revised (PCL-R) in Caucasian and African-
American participants. Psychological Assessment, 13, 531-542.

Cooke, D.J., \& Michie, C. (1997). An item response theory evaluation of Hare's Psychopathy Checklist. Psychological Assessment, 9, 2-13.

Cooke, D.J., \& Michie, C. (1999).

Psychopathy across cultures. Journal of Abnormal Psychology, 108, 58-68.

Cooke, D.J., \& Michie, C. (2001). Refining the construct of psychopathy: Towards a hierarchical model. Psychological Assessment, 13, 171-188.

Cooke, D.J., Michie, C., Hart, S.D., \& Clark, D.A. (2004). Reconstructing psychopathy: Clarifying the significance of antisocial and socially deviant behavior in the diagnosis of psychopathic personality disorder. Journal of Personality Disorders, 18, 337-356.

Cooke, D.J., Michie, C. , \& Skeem, J. (2007). Understanding the structure of the Psychopathy Checklist-Revised: An exploration of methodological confusion. British Journal of Psychiatry, 190 (Suppl. 49), s39-s50.

Cornell, D.G., Warren, J., Hawk, G., Stafford, E., Oram, G., \& Pine, D. (1996). 
Psychopathy in instrumental and reactive violent offenders. Journal of Consulting and Clinical Psychology, 64, 783-790.

Corrado, R., Vincent, G., Hart, S., \& Cohen, I. (2004). Predictive validity of the PCL:YV for general and violent redivism. Behavioral Sciences and the Law, 22, 522.

Cronbach, L.J., \& Meehl, P.E. (1955).

Construct validity of psychological tests. Psychological Bulletin, 52, 281-302.

Das, J., \& Ruiter, C. de (2003). Youth

Psychopathic traits Inventory: geautoriseerde Nederlandse vertaling. [Youth Psychopathic traits Inventory: Authorized Dutch translation].

Unpublished research version.

Das, J., de Ruiter, C., Lodewijks, H., \& Doreleijers, T. (2007). Predictive validity of the Dutch PCL:YV for institutional disruptive behavior: Findings from two samples of male adolescents in a juvenile justice treatment institution. Behavioral Sciences and the Law, 25, 739-755.

Das, J., Ruiter, C. de, Doreleijers, Th., \& Hillege, S. (accepted). Reliability and construct validity of the Dutch Psychopathy Checklist: Youth Version:
Findings from a sample of male adolescents in a juvenile justice treatment institution. Assessment.

Derksen, J., Dijk, J., van, \& Cornelissen, A. (2003). De Nederlandse adolescentenversie van de MMPI. [The Dutch language version of the MMPI for adolescents.] De Psycholoog, 38, 304-311.

Derksen, J., Mey, H. de, Sloore, H. \& Hellenbosch, G.M. (1995). MMPI-2: Handleiding bij afname, scoring en interpretatie. [Manual for the assessment, scoring and interpretation of the Dutch MMPI-2]. Nijmegen: Pen Tests Publishers.

Dijk, J.S. van, Cornelissen, A.J., \& Derksen, J. (2000) MMPI-A, handleiding bij afname, scoring en interpretatie. [Manual for the assessment, scoring and interpretation of the Dutch MMPI-A.] Nijmegen: PEN Tests Publishers.

Dolan, M.C., \& Anderson, I.M. (2003). The relationship between serotonergic function and the Psychopathy Checklist: Screening Version. Journal of Psychopharmacology, 17, 216-222.

Dolan, M.C., \& Rennie, C.E. (2007). The relationship between psychopathic traits measured by the Youth Psychopathic 
traits Inventory and psychopathology in a UK sample of conduct disordered boys. Journal of Adolescence, 30, 601-611.

Edens, J.F., Buffington-Vollum, J.K., Colwell, K.W., Johnson, D.W., \& Johnson, J.K. (2002). Psychopathy and institutional misbehavior among incarcerated sex offenders: A comparison of the Psychopathy Checklist-Revised and the Personality Assessment Inventory. International Journal of Forensic Mental Health, 1, 49-58.

Edens, J.F., \& Cahill, M.A. (2007).

Psychopathy in adolescence and criminal recidivism in young adulthood: Longitudinal results from a multiethnic sample of youthful offenders. Assessment 14, 57-64.

Edens, J.F., \& Campbell, J.S. (2007).

Identifying youths at risk for institutional misconduct: A metaanalysis investigation of the Psychopathy Checklist Measures. Psychiatric Services, 4, 13-27.

Edens, J.F. , Campbell, J.S, \& Weir, J.M. (2007). Youth psychopathy and criminal recidivism: A meta-analysis of the Psychopathy Checklist Measures. Law and Human Behavior, 31, 53-75.
Edens, J.F., Marcus, D.K., Lilienfeld, S.O., \& Poythress, N.G. (2006).

Psychopathis, not psychopath: Taxometric evidence for the dimensional structure of psychopathy. Journal of Abnormal Psychology, 115, 131-144.

Edens, J.F., Petrila, J., \& BuffingtonVollum, J.K. (2001). Psychopathy and the death penalty: Can the Psychopathy Checklist-Revised identify offenders who represent "a continuing threat to society"? The Journal of Psychiatry \& Law, 29, 433-481.

Edens, J.F., Poythress, N.G., \& Lilienfeld, S.O. (1999). Identifying inmates at risk for disciplinary infractions: A comparison of two measures of psychopathy. Behavioral Sciences and the Law, 17, 435-443.

Edens, J.F., Skeem, J.L., Cruise, K.R., \& Cauffman, E. (2001). Assessment of 'juvenile psychopathy' and its association with violence: A critical review. Behavioral Sciences and the Law, 19, 5380.

Edens, J.F., Skopp, N.A., \& Cahill, M.A. (2008). Psychopathic features moderate the relationship between harsh and inconsistent parental discipline and adolescent antisocial behavior. Journal of 
Clinical Child \& Adolescent Psychology, $37,472-476$.

Edens, J.F. \& Vincent, G.M. (in press). Juvenile psychopathy: A clinical construct in need of restraint? Journal of Forensic Psychology Practice.

Essau, C. A., Sasagawa, S., \& Frick, P. J. (2006). Callous-unemotional traits in a community sample of adolescents. Assessment, 13, 454-469.

Falkenbach, D.M., Poythress, N.G., \& Heide, K.M. (2003). Psychopathic features in a juvenile diversion population: Reliability and predictive validity of two self-report measures. Behavioral Sciences and the Law, 21, 787-805.

Farrington, D.P. (2005). The importance of child and adolescent psychopathy. Journal of Abnormal Child Psychology, 33, 489-497.

Fiske, D.W., \& Campbell, D.T. (1992).

Citations do not solve problems.

Psychological Bulletin, 112, 393-395.

Fleiss, J.L. (1986). The design and analysis of clinical experiments. New York: Wiley.
Flor, H., Birbaumer, N., Hermann, C., Ziegler, S., \& Patrick, C. (2002). Aversive Pavlovian conditioning in psychopaths: Peripheral and central correlates. Psychophysiology, 39, 505-518.

Forth, A.E., Brown, S.L., Hart, S.D., \& Hare, R.D. (1996). The assessment of psychopathy in male and female noncriminals: Reliability and validity. Personality and Individual Differences, 20, 531-543.

Forth, A.E., \& Burke, H.C. (1998).

Psychopathy in adolescence: Assessment, violence, and developmental precursors. In D.J. Cooke, A.E. Forth, \& R.D. Hare (Eds.), Psychopathy: Theory, research, and implications for society (pp. 205-229).

Dordrecht, The Netherlands: Kluwer.

Forth, A.E., Hart, S.D., \& Hare, R.D. (1990). Assessment of psychopathy in male young offenders. Psychological Assessment: A Journal of Consulting and Clinical Psychology, 2, 342-344.

Forth, A. E., Kosson, D., \& Hare, R. D. (2003). Psychopathy Checklist: Youth Version. Toronto: Multi-Health System.

Forth, A.E., \& Mailloux, D.L. (2000). Psychopathy in youth: What do we know? 
In C.B. Gacono (Ed.), The clinical and forensic assessment of psychopathy: $A$ practitioner's guide (pp. 25-54). Mahwah, NJ: Lawrence Erlbaum.

Frick, P.J. (1998). Conduct disorders and severe antisocial behavior. New York: Plenum.

Frick, P.J. (2000). The problems of internal validation without a theoretical context: The different conceptual underpinnings of psychopathy and the disruptive behavior disorder criteria. Psychological Assessment, 12, 451-465.

Frick, P.J. (2003). The Inventory of Callous-Unemotional Traits. Unpublished rating scale. University of New Orleans.

Frick, P.J., Barry, C.T., \& Bodin, S.D. (2000). Applying the concept of psychopathy to children: Implications for the assessment of antisocial youth. In C. B. Gacono (Ed.), The clinical and forensic assessment of psychopathy: $A$ practitioner's guide (pp. 3-24). Mahwah. NJ: Lawrence Erlbaum.

Frick, P.J., Bodin, S.D., \& Barry, C.T. (2000). Psychopathic traits and conduct problems in community and clinicreferred samples of children: Further development of the Psychopathy Screening Device. Psychological Assessment, 12, 382-393.

Frick, P.J., Cornell, A.H., Barry, C.T., Bodin, S.D., \& Dane, H.A. (2003). Callousunemotional traits and conduct problems in the prediction of conduct problem severity, aggression, and self-report of delinquency. Journal of Abnormal Child Psychology, 31, 457-470.

Frick, P.J., \& Ellis, M. (1999). Callousunemotional traits and subtypes of conduct disorder. Clinical Child and Family Psychology Review, 2, 149-168.

Frick, P.J. \& Hare, R.D. (2002). Antisocial Process Screening Device. Toronto, Canada: Multi-Health Systems.

Frick, P.J., Kimonis, E.R., Dandreaux, D.M., \& Farell, J.M. (2003). The 4 year stability of psychopathic traits in nonreferred youth. Behavioral Sciences and the Law, 21, 713-736.

Frick, P.J., Lilienfeld, S.O., Ellis, M.L, Loney, B.R., \& Silverthorn, P. (1999). The association between anxiety and psychopathy dimensions in children. Journal of Abnormal Child Psychology, 27, 381-390. 
Friedman, A.F., Lewak, R., Nichols, D.S., \& Webb, J.T. (2001). Psychological Assessment with the MMPI-2. Maywah, New Jersey: Lawrence Erlbaum.

Gendreau, P., Goggin, C., \& Smith, P. (2002). Is the PCL-R really the "unparalleled" measure of offender risk? A lesson in knowledge cumulation. Criminal Justice and Behavior, 29, 397426.

Glick, B., \& Goldstein, A.P. (1987). Aggression Replacement Training. Journal of Counseling \& Development, 65, 356-362.

Grafman, J., Schwab, K., Warden, D., Pridgen, B.S., \& Brown, H.R. (1996). Frontal lobe injuries, violence, and aggression: A report of Vietnam head injury study. Neurology, 46, 1231-1238.

Grann, M. (2000). The PCL-R and gender. European Journal of Psychological Assessment, 16, 147-149.

Grann, M., Långström, N., Tengström, A., \& Stålenheim, E.G. (1998). Reliability of file-based retrospective ratings of psychopathy with the PCL-R. Journal of Personality Assessment, 70, 416-426.

Gray, N.S., Hill, C., McGleish, A.,
Timmons, D., MacCulloch, M.J., \& Snowden, R.J. (2003). Prediction of violence and self-harm in mentally disordered offenders: A prospective study of the efficacy of HCR-20, PCL-R, and psychiatric symptomatology. Journal of Consulting and Clinical Psychology, 71, 443-451.

Green, S.B., Lissitz, R. W., \& Mulaik, S.A. (1977). Limitations of coefficient alpha as an index of unidimensionality. Educational and Psychological Measurement, 37, 827-838.

Gretton, H., Hare, R.D., \& Catchpole, R. (2004). Psychopathy and offending from adolescence to adulthood: A ten-year follow-up. Journal of Consulting and Clinical Psychology, 72, 636-645.

Gretton, H.M., McBride, M., Hare, R.D., O’Shaughnessy, R., \& Kumka, G. (2001). Psychopathy and recidivism in adolescent sex offenders. Criminal Justice and Behavior, 28, 427-449.

Guy, L.S., Edens, J.F., Anthony, C, \& Douglas, K.S. (2005). Does psychopathy predict institutional misconduct among adults? A meta-analytic investigation. Journal of Consulting and Clinical Psychology, 73, 1056-1064. 
Hall, J.R., Benning, S.D., \& Patrick, C.J. (2004). Criterion-related validity of the three-factor model of psychopathy: Personality, behaviour, and adaptive functioning. Assessment, 11, 4-16.

Hare, R.D. (1991). The Hare Psychopathy Checklist - Revised. Toronto, Ontario

Canada: Multi-Health Systems.

Hare, R.D. (1998). Without conscience: The disturbing world of the psychopaths among us. New York: Guilford Press.

Hare, R.D. (2003). Manual for the Revised Psychopathy Checklist (2nd ed.). Toronto, Ontario, Canada: Multi-Health Systems.

Hare, R.D., Harpur, T.J., Hakstian, A.R., Forth, A.E., Hart, S.D., \& Newman, J.P. (1990). The Revised Psychopathy Checklist: Descriptive statistics, reliability and factor structure. Psychological Assessment: A Journal of Consulting and Clinical Psychology, 2, 338-341.

Hare, R.D., Hart, S.D., Forth, A.E., Harpur, T.J., \& Williamson, S.E. (1998). Psychopathic personality characteristics: Development of a criteria set for use in the DSM-IV antisocial personality disorder field trial. In T. A. Widiger, A.
J. Frances, H. A. Pincus, R. Ross, M. B. First, W. Davis, \& M. Kline (Eds.), DSMIV Sourcebook (pp.331-341). Washington, DC: American Psychiatric Association.

Hare, R.D., McPherson, L.E., \& Forth, A.E. (1988). Male psychopaths and their criminal careers. Journal of Consulting and Clinical Psychology, 56, 710-714.

Hare, R.D., \& Neumann, C.S. (2005). The structure of psychopathy. Current Psychiatry Reports, 7, 1-32.

Harpur, T.J., Hakstian, R., \& Hare, R.D. (1988). Factor structure of the Psychopathy Checklist. Journal of Consulting and Clinical Psychology, 56, 741-747.

Harpur, T.J., Hare, R.D., Hakstian, R. (1989). Two-factor conceptualization of psychopathy: Construct validity and assessment implications. Psychological Assessment: A Journal of Consulting and Clinical Psychology, 1, 6-17.

Harris, G.T., Rice, M.E., \& Quinsey, V.L. (1994). Psychopathy as a taxon: Evidence that psychopaths are a discrete class. Journal of Consulting and Clinical Psychology, 62, 387-397. 
Hart, S.D. (1998). The role of psychopathy in assessing risk for violence: Conceptual and methodological issues. Legal and Criminological Psychology, 3, 121-137.

Hart, S.D., Cox, D.N., \& Hare, R.D. (1995). Manual for the Psychopathy

Checklist: Screening Version (PCL: SV).

Toronto: Multi-Health Systems.

Hart, S.D., Hare, R.D., \& Forth, A.E. (1994). Psychopathy as a risk marker for violence: Development and validation of a screening version of the Revised Psychopathy Checklist. In J. Monahan \& H. J. Steadman (Eds.), Violence and mental disorder: Developments in risk assessment (pp. 81-99). Chicago, IL:

University of Chicago Press.

Hart, S.D., Watt, K.A., \& Vincent, G.M. (2002). Commentary on Seagrave and Grisso: Impressions of the state of the art. Law and Human Behavior, 26, 241-245.

Harvey, P.D., Stokes, J.L., Lord, J., Pogge, D.L. (1996). Neurocognitive and personality assessment of adolescent substance abusers: A multidimensional approach. Assessment, 3, 241-253.

Hemphill, J.F., Hare, R.D., \& Wong, S. (1998). Psychopathy and recidivism:
A review. Legal and Criminological

Psychology, 3, 141-172.

Hemphill, J.F., Hart, S.D., \& Hare, R.D. (1994). Psychopathy and substance use. Journal of Personality Disorders, 8, 169180.

Hicks, M., Rogers, R., \& Cashel, M. (2000). Predictions of violent and total infractions among institutionalized male juvenile offenders. Journal of the American Academy of Psychiatry and the Law, 28, 183-190.

Hildebrand, M., Hesper, B.L., Spreem, M., \&Nijman, H.L.I. (2005). De waarde van gestructureerde risicotaxatie en van de diagnose psychopathie: een onderzoek naar de betrouwbaarheid en predictieve validiteit van de HCR-20, HKT-30 en PCL-R [The value of structured risk assessment and the diagnosis of psychopathy: A study of the reliability and predictive validity of the HCR-20, HKT-30 and PCL-R]. Amsterdam: Sphinex $\&$ Industrie.

Hildebrand, M., \& Ruiter, C. de (2004). PCL-R psychopathy and its relation to DSM-IV Axis I and Axis II disorders in a sample of male forensic psychiatric patients in the Netherlands. International 
Journal of Law and Psychiatry, 27, 233248.

Hildebrand, M., Ruiter, C. de, \& Beek, D.J., van (2000). SVR-20: Sexual Violence Risk-20. Dr. Henri van der Hoeven Stichting: Utrecht.

Hildebrand, M., Ruiter, C. de, \& Nijman, H. (2004). PCL-R psychopathy predicts disruptive behavior among male offenders in a Dutch forensic psychiatric hospital. Journal of Interpersonal Violence, 19, 13-29.

Hildebrand, M., Ruiter, C. de, \& Vogel, V. de (2004). Psychopathy and sexual deviance in treated rapists: Association with sexual and non-sexual recidivism. Sexual Abuse: A Journal of Research and Treatment, 16, 1-24.

Hildebrand, M., Ruiter, C. de, \& Zaane, B., van. Psychopathy and change in dynamic risk factors during inpatient treatment. Submitted for publication.

Hill, C., Neumann, C.S., \& Rogers, R. (2004). Confirmatory factor analysis of the Psychopathy Checklist: Screening Version (PCL: SV) in offenders with axis I disorders. Psychological Assessment, 16, 90-95.
Hobson, J., Shine, J., \& Roberts, R. (2000). How do psychopaths behave in a prison therapeutic community? Psychology, Crime, and Law, 6, 139-154.

Hu, L., \& Bentler, P.M. (1999). Cutoff criterion for fit indexes in covariance structure analysis: Conventional criteria versus new alternatives. Structural Equation Modeling, 6, 1-55.

Hulin, C.L. (1987). A psychometric theory of evaluations of item and scale translations: Fidelity across languages. Journal of Cross-Cultural Psychology, 18, 115-142.

Inspectie Jeugdzorg (2007). Veiligheid in justitiële jeugdinrichtingen: opdracht met risico's. [Safety in juvenile justice institutions: a risky assignment]. Utrecht: Inspectie Jeugdzorg.

Johansson, P., Andershed, H., Kerr, M., \& Levander, S. (2002). On the operationalization of psychopathy: Further support for a three-faceted personality oriented model. Acta Psychiatrica Scandinavica, 106, 81-85.

Johnstone, L. \& Cooke, D.J. (2004).

Psychopathic like traits in childhood: Conceptual and measurement concerns. 
Behavioral Science and the Law, 22, 103125.

Jones, S., Cauffman, E., Miller, J.D., \& Mulvey, E. (2006). Investigating different factor structures of the Psychopathy

Checklist: Youth Version (PCL:YV):

Confirmatory factor analytic findings.

Psychological Assessment, 18, 33-48.

Jong, C.A.J. de, Brink, W. van den, \& Jansma, A. (2000). ICL-R: Handleiding

bij de vernieuwde versie van de Interpersonal Checklist (ICL). [Manual of the revised Dutch version of the Interperonal Checklist (ICL)]. Sint

Oedenrode, The Netherlands: Novadic.

Knight, R.A., \& Sims-Knight, J.E.

(2003) The developmental antecedents of sexual coercion against women: Testing alternative hypotheses with structural equation modeling. Annals of the New York Academy of Sciences, 989, 72-85.

Kosson, D.S., Cyterski, T.D., Steuerwald, B.L., Neumann, C. S., \& Walker-

Matthews, S. (2002). The reliability and validity of the Psychopathy Checklist: Youth Version (PCL: YV) in nonincarcerated adolescent males. Psychological Assessment, 14, 97-109.
Kotler, J.S., \& McMahon, R.J. (2005). Child psychopathy: Theories, measurement, and relations with the development and persistence of conduct problems. Clinical Child and Family Psychology Review, 8, 291-325.

LaForge, R., \& Suczek, R. F. (1955). The interpersonal dimension of personality: III. An interpersonal checklist. Journal of Personality, 24, 94-112.

Lahey, B.B., Loeber, R., Quay, H.C., Applegate, B., Schaffer, D., Waldman, I., Hart, E.L., McBurnett, K., Frick, P.J., Jensen, P.S., Dulcan, M.K., Canino, G., \& Bird, H. (1998). Validity of DSM-IV subtypes of conduct disorder based on age of onset. Journal of the American Academy of Child and Adolescent Psychiatry, 37, 435-442.

Långström, N., \& Grann, M. (2002). Psychopathy and violent recidivism among young criminal offenders. Acta Psychiatrica Scandinavica, 106, 86-92. Leary, T. (1957). Interpersonal diagnosis of personality. New York: Ronald.

Lee, Z., Vincent, G.M., Hart, S.D., \& Corrado, R.R. (2003). The validity of the Antisocial Process Screening Device as 
a self-report measure of psychopathy in adolescent offenders. Behavioral Sciences and the Law, 21, 771-786.

Levenston, G.K., Patrick, C.J., Bradley, M.M., \& Lang, P.J. (2000). The psychopath as observer: Emotion and attention in picture processing. Journal of Abnormal Psychology, 109, 373-385.

Lewis, D.O., Yeager, C.A., CobhamPortorreal, C.S., Klein, N., Showater, C., \& Anthony, A. (1991). A followup of female delinquents: Maternal contributions to the perpetuation of deviance. Journal of the American Academy of Child and Adolescent Psychiatry, 30, 197-201.

Lodewijks, H.P.B., Doreleijers, Th., Ruiter, C. de \& Wit-Grouls, H. de (2001). Gestructureerde taxatie van geweldsrisico bij jongeren (geautoriseerde vertaling en onderzoeksversie van de SAVRY). [Structured assessment of violence risk in youth (authorized translation and research version of the SAVRY)]. Eefde: Rentray.

Loney, B.R., Taylor, J., Butler, M.A., \& Iacono, W.G. (2007). Adolescent psychopathy features: 6-year temporal stability and the prediction of externalizing symptoms during the transition to adulthood. Aggressive Behavior, 33, 242-252.

Lorenz, A.R., \& Newman, J.P. (2002).

Deficient response modulation and emotion processing in low-anxious Caucasian psychopathic offenders: Results from a lexical decision task. Emotion, 2, 91-104.

Luteijn, F., Dijk, H. van, \& Ploeg, F.A.E., van der (1989). Handleiding bij de NPV-J. [Manual for the NPV-J]. Lisse: Harcourt Test Publishers.

Lynam, D.R. (1996). Early identification of chronic offenders: Who is the fledgling psychopath? Psychological Bulletin, 120, 209-234.

Lynam, D.R. (1997). Pursuing the psychopath: Capturing the fledgling psychopath in a nomological net. Journal of Abnormal Psychology, 106, 425-438.

Lynam, D.R., Caspi, A., Moffit, T.E., Raine, A., Loeber, R., \& StouthamerLoeber, M. (2005). Adolescent psychopathy and the Big 5: Results from two samples. Journal of Abnormal Child Psychology, 33, 431-443. 
Lynam, D.R., Caspi, A., Moffitt, T.E.,

Loeber, R., \& Stouthamer-Loeber, M.

(2007). Longitudinal evidence that

psychopathy scores in early adolescence

predict adult psychopathy. Journal of

Abnormal Psychology, 116, 155-165.

Lynam, D.R. \& Gudonis, L. (2005). The

development of psychopathy. Annual

Review of Clinical Psychology, 1, 381-407.

Mailloux, D.L., Forth, A.E., \& Kroner,

D.G. (1997). Psychopathy and substance

use in adolescent male offender.

Psychological Reports, 80, 529-530.

Marsee, M.A., Silverthorn, P., \&

Frick, P.J. (2005). The association of

psychopathic traits with aggression and

delinquency in non-referred boys and

girls. Behavioral Sciences and the Law,

23, 803-817.

McGraw, K.O., \& Wong, S.P. (1996).

Forming inferences about some intraclass

correlation coefficients. Psychological

Methods, 1, 30-46.

Mitchell, D.G.V., Colledge, E., Leonard, A., \& Blair, R.J.R. (2002). Risky decisions and response reversal: Is there evidence of orbitofrontal cortex dysfunction in psychopathic individuals?
Neuropsychologia, 40, 2013-2022.

Moffit, T.E. (1993). Adolescence-limited and life-course persistent antisocial behavior: A developmental taxonomy. Psychological Review, 100, 674-701.

Morrissey, C., Hogue, T.E., Mooney, P., Allen, C., Johnston, S., Hollin, C., Lindsay, W.R., \& Taylor, J.L. (2007). Predictive validity of the PCL-R in offenders with intellectual disability in a high secure hospital setting: Institutional aggression. Journal of Forensic

Psychiatry \& Psychology, 18, 1-15.

Morrissey, C., Hogue, T.E., Mooney,

P., Lindsay, W.R., Steptoe, L.,

Taylor, J., \& Johnston, S. (2005).

Applicability, reliability and validity

of the Psychopathy Checklist-Revised in

offenders with intellectual disabilities:

Some initial findings. International

Journal of Forensic Mental Health, 4, 207-220.

Morrissey, C., Mooney, P., Hogue, T.E., Lindsay, W.R., \& Taylor, J.L. (2007). Predictive validity of the PCL-R for offenders with intellectual disability in a high security hospital: Treatment progress. Journal of Intellectual \& Developmental Disability, 32, 125-133. 
Murrie, D.C., \& Cornell, D.G. (2002).

Psychopathy screening of incarcerated

juveniles: A comparison of measures.

Psychological Assessment, 14, 390-396.

Murrie, D.C., Cornell, D.G., Kaplan, S., McConville, D., \& Levy-Elkon, A. (2004).

Psychopathy scores and violence among juvenile offenders: A multi-measure study. Behavioral Sciences and the Law, 22, 49-67.

Murrie, D.C., Marcus, D.K., Douglas, K.S., Lee, Z., Salekin, R.T., \& Vincent, G. (2007). Youth with psychopathy features are not a discrete class: A taxometric analysis. Journal of Child Psychology and Psychiatry, 48, 714-723.

Muthen, L.K., \& Muthen, B.O. (1998). Mplus user's guide. Los Angeles, CA: Muthen \& Muthen.

Myers, W.C., Burket, R.C., \& Harris, H.E. (1995). Adolescent psychopathy in relation to delinquent behaviours, conduct disorder, and personality disorders. Journal of Forensic Sciences, 40, 435-439.

Neumann, C.S., Kosson, D.S., Forth, A.E., \& Hare, R.D. (2006). Factor structure of the Hare Psychopathy Checklist: Youth Version in incarcerated adolescents.
Psychological Assessment, 18, 142-154.

Neumann, C.S., Vitacco, M.J., Hare, R.D., \& Wupperman, P. (2005). Reconstructing the "reconstruction" of psychopathy: A comment on Cooke, Michie, Hart, and Clarke. Journal of Personality Disorders, 19, 624-640.

Newman, J.P. (1998). Psychopathic behaviour: An information processing perspective. In. D.J. Cooke, A.E. Forth, \& R.D. Hare (Eds.), Psychopathy: Theory, research and implications for society (pp. 81-104). Dordrecht, The Netherlands: Kluwer.

Newman, J.P., \& Kosson, D.S. (1986). Passive avoidance learning in psychopathic and nonpsychopathic offenders. Journal of Abnormal Psychology, 95, 252-256.

Newman, J.P., \& Schmitt, W. (1998). Passive avoidance in psychopathic offenders: A replication and extension. Journal of Abnormal Psychology, 107, 527-532.

Newman, J.P., Schmitt, W., \& Voss, W. (1997). The impact of motivationally neutral cues on psychopathic individuals: Assessing the generality of the response 
modulation hypothesis. Journal of

Abnormal Psychology, 106, 563-575.

Nicholls, T.L., Ogloff, J.R.P., Brink, J., \& Spidel, A. (2005). Psychopathy in women: A review of its clinical usefulness for assessing risk for aggression and criminality. Behavioral Sciences and the Law, 23, 779-802.

Nicholls, T.L., \& Petrila, J. (2005).

Gender and psychopathy: An overview of important issues and introduction to the special issue. Behavioral Sciences and the Law, 23,_729-741.

Nunnally, J., Bernstein, I. (1994).

Psychometric theory. New York: McGrawHill.

O’Brien, B.S., \& Frick, P.J. (1996). Reward dominance: Associations with anxiety, conduct problems, and psychopathy in children. Journal of Abnormal Child Psychology, 24, 223-240.

O’Neill, M.L., Lidz, V., \& Heilbrun, K. (2003). Predictors and correlates of psychopathic characteristics in substance abusing adolescents. International Journal of Forensic Mental Health, 2, 3545.
Odgers, C.L., \& Moretti, M.M. (2002). Aggressive and antisocial girls: Research update and challenges. International Journal of Forensic Mental Health, 1, 103-119.

Odgers, C.L., Repucci, N.D., \& Moretti, M. (2005). Nipping psychopathy in the bud: An examination of the convergent, predictive and theoretical utility of the PCL:YV among adolescent girls. Behavioral Sciences and the Law, 23, 743763.

Ogloff, J.R., \& Wong, S. (1990).

Electrodermal and cardiovascular evidence of a coping response in psychopaths. Criminal Justice and Behavior, 17, 231-245.

Ogloff, J., Wong, S., \& Greenwood, A. (1990). Treating criminal psychopaths in a therapeutic community program. Behavioral Sciences and the Law, 8, 181190.

Olver, M.E., \& Wong, S.C.P. (2006).

Psychopathy, sexual deviance, and recidivism among sex offenders. Sexual Abuse: A Journal of Research and Treatment, 18, 65-82. 
Oxford, M., Cavell, T.A., \& Hughes, J.N. (2003). Callous/unemotional traits moderate the relation between ineffective parenting and child externalizing problems: A partial replication and extension. Journal of Clinical Child and Adolescent Psychology, 32, 577-585.

Patrick, C.J., Bradley, M.M., \& Lang, P.J. (1993). Emotion in the criminal psychopath: Startle reflex modulation. Journal of Abnormal Psychology, 102, 82-92.

Pepler, D.J., Madsen, K.C., Webster, C., \& Levene, K.S. (Eds.). (2005). The development and treatment of girlhood aggression. Mahwah, NJ: Lawrence Erlbaum Associates.

Petrila, J., \& Skeem. J.L. (2003). An introduction to the special issues on juvenile psychopathy and some reflections on the current debate. Behavioral Sciences and the Law, 21, 689-694.

Porter, S., Fairweather, D., Drugge, J., Hervé, H., Birt, A., \& Boer, D.P. (2000). Profiles of psychopathy in incarcerated sexual offenders. Criminal Justice and Behavior, 27, 216-233.
Porter, S., Woodworth, M., Earle, J., Drugge, J., \& Boer, D. (2003). Characteristics of sexual homicides committed by psychopathic and nonpsychopathic offenders. Law and Human Behavior, 27, 459-470.

Poythress, N.G., Dembo, R., Wareham, J., \& Greenbaum, P.E. (2006). Construct validity of the Youth Psychopathic Traits Inventory (YPI) and the Antisocial Process Screening Device (APSD) with justice involved adolescents. Criminal Justice and Behavior, 33, 26-55.

Raine, A. (1993). The psychopathology of crime: Criminal behavior as a clinical disorder. San Diego, CA: Academic Press.

Raine, A. (2002). Biosocial studies of antisocial and violent behavior in children and adults: A review. Journal of Abnormal Child Psychology, 30, 311-326.

Rice, M.E., \& Harris, G.T. (1997). Cross validation and extension of the Violence Risk Appraisal Guide for child molesters and rapists. Law and Human Behavior, 21, 231-241.

Rice, M.E., Harris, G.T., \& Cormier, C.A. (1992). An evaluation of a maximumsecurity therapeutic community 
for psychopaths and other mentally disordered offenders. Law and Human Behavior, 16, 399-412.

Richards, H.J., Casey, J.O., \& Lucente, S.W. (2003). Psychopathy and treatment response in incarcerated female substance abusers. Criminal Justice and Behavior, 30, 251-276.

Robins, L.N. (1986). The consequences of conduct disorder in girls. In D. Olweus, J. Block, \& M. Radke-Yarrow (Eds.), Development of antisocial and prosocial behavior: Research, theories, and issues (pp. 385-414). Orlando, FL: Academic Press.

Rogers, R., Johansen, J., Chang, J.J., \& Salekin, R.T. (1997). Predictors of adolescent psychopathy: Oppositional and conduct-disordered symptoms. Journal of the American Academy of Psychiatry and the Law, 25, 261-271.

Ruiter, C. de, Das, \& Domburgh, L., van (2003). Antisocial Process Screening Device: Geautoriseerde Nederlandse vertaling [Antisocial Process Screening Device: Authorized Dutch Translation]. Unpublished Research Version.

Ruiter, C. de, Kuin, N., Vries, J. de, \& Das, J (2002). Psychopathie Checklist: Jeugd Versie: Geautoriseerde Nederlandse vertaling. [Psychopathy Checklist: Youth Version: Authorized Dutch translation]. Unpublished research version.

Salekin, R.T. (2008). Psychopathy and recidivism from mid-adolescence to young adulthood: Cumulating legal problems and limiting life opportunities. Journal of Abnormal Psychology, 117, 386-395.

Salekin, R.T., Brannen, D.N., Zalot, A.A., Leistico, A., \& C.S. (2006). Factor structure of psychopathy in youth: Testing the applicability of the new four-factor model. Criminal Justice and Behavior 33, 135-157.

Salekin, R.T., \& Frick, P.J. (2005).

Psychopathy in children and adolescents: The need for a developmental perspective. Journal of Abnormal Child Psychology, 33, 403-409.

Salekin, R. T., Leistico, A. R., Trobst, K. K., Schrum, C. L., \& Lochman, J. E. (2005). Adolescent Psychopathy and personality theory - the interpersonal circumplex: Expanding evidence of a nomological net. Journal of Abnormal Child Psychology, 33, 445-460. 
Salekin, R.T., Neumann, C.C., Leistico, A.R., DiCicco, T., \& Duros, R. (2004). Psychopathy and comorbidity in an offender sample: Taking a closer look at the potential importance of psychopathy over disruptive behavior disorders. Journal of Abnormal Psychology, 113, 416-427.

Salekin, R., Rogers, R., \& Sewell, K. (1996). A review and meta-analysis of the Psychopathy Checklist-Revised: Predictive validity of dangerousness. Clinical Psychology: Science and Practice, 3, 203-215.

Salekin, R., Rogers, R., Ustad, K.L., \& Sewell, K. (1998). Psychopathy and recidivism among female inmates. Law and Human Behavior, 22, 109-128.

Samejima, F. (1997). Graded response model. In W. J. van der Linden \& R. K. Hambleton (Eds.), Handbook of modern item response theory (pp. 85-100). New York: Springer-Verlag.

Schmidt, F., McKinnan, L., Chattha, H.K., \& Brownlee, K. (2006). Concurrent and predictive validity of the Psychopathy Checklist: Youth Version across gender and ethnicity. Psychological Assessment, 18, 393-401.
Schreurs, P.J.G., Willige, G. van de, Tellegen, B. \& Brosschot, J.F. (1988). Handleiding Utrechtse Copinglijst: UCL. [Manual for the Utrecht Coping Questionnaire]. Lisse: Swets \& Zeitlinger. Schrum, C.L. \& Salekin, R.T. (2006). Psychopathy in adolescent female offenders: An Item Response Theory analysis of the Psychopathy Checklist: Youth Version. Behavioral Sciences and the Law, 24, 39-63.

Seagrave, D., \& Grisso, T. (2002). Adolescent development and the measurement of juvenile psychopathy. Law and Human Behavior, 26, 219-239.

Seto, M.C., \& Barbaree, H.E. (1999). Psychopathy, treatment behavior, and sex offenders' recidivism. Journal of Interpersonal Violence, 14, 1235-1248.

Shrout, P.E. \& Fleiss, J.L. (1979)

Intraclass Correlations: Uses in assessing rater reliability. Psychological Bulletin, $86,420-428$.

Silverthorn, P., \& Frick, P.J. (1999).

Developmental pathways to antisocial behavior: The delayed-onset pathway in girls. Development and Psychopathology, 11, 101-126. 
Silverthorn, P., Frick, P.J., \& Reynolds, R. (2001). Timing of onset and correlates of severe conduct problems in adjudicated girls and boys. Journal of Psychopathology and Behavioral Assessment, 23, 171-181.

Skeem, J.L., \& Cauffman, E. (2003). Views of the downward extension: Comparing the Youth Version of the Psychopathy Checklist with the Youth Psychopathic traits Inventory. Behavioral Sciences and the Law, 21, 737-770.

Skeem, J.L., Monahan, J., \& Mulvey, E.P. (2002). Psychopathy, treatment involvement, and subsequent violence among civil psychiatric patients. Law and Human Behavior, 26, 577-603.

Skeem, J.L., Mulvey, E.P., \& Grisso, T. (2003). Applicability of traditional and revised models of psychopathy to the Psychopathy Checklist: Screening Version. Psychological Assessment, 15, 41-55.

Slot, W., \& Spanjaard, H. (1999).

Competentievergroting in de residentiële jeugdzorg. Hulpverlening voor kinderen en jongeren in tehuizen. [Improving competence in residential juvenile welfare. Mental health care for children and adolescents in residential homes.] Baarn: Intro.

Smith, S.S., \& Newman, J.P. (1990). Alcohol and drug abuse/dependence disorders in psychopathic and nonpsychopathic criminal offenders. Journal of Abnormal Psychology, 99, 430-439.

Spain, S.E., Douglas, K.S., Poythress, N.G., \& Epstein, M. (2004). The relationship between psychopathic features, violence and treatment outcome: The comparison of three youth measures of psychopathic features. Behavioral Sciences and the Law, 22, 85-102.

Stevens, D., Charman, T., \& Blair, R.J. (2001). Recognition of emotion in facial expressions and vocal tones in children with psychopathic tendencies. Journal of Genetic Psychology, 162, 201-211.

Stinson, J.D., Becker, J.V., \& Tromp, A. (2005). A preliminary study of findings of psychopathy and affective disorders in adult sex offenders. International Journal of Law and Psychiatry, 28, 637649.

Taylor, J., Lang, A.R. (2006). Psychopathy and substance use disorders. In C.J. Patrick 
(Eds.), Handbook of psychopathy (pp. 495511). New York: Guilford.

Thissen, D., Chen, W.H., \& Bock, R.D. (2003). Multilog (version 7) [Computer software]. Lincolnwood, IL: Scientific Software International.

Vaughn, M.G., \& Howard, M.O. (2005).

Self-report measures of juvenile

psychopathic personality traits: A comparative review. Journal of Emotional and Behavioral Disorders, 13, 152-162.

Verona, E., Hicks, B.M., \& Patrick, C.J. (2005). Psychopathy and suicidal behavior in female offenders: Mediating influences of personality and abuse history. Journal of Consulting and Clinical Psychology, 73, 1065-1073.

Verona, E., Patrick, C.J., \& Joiner, T.T. (2001). Psychopathy, antisocial personality, and suicide risk. Journal of Abnormal Psychology, 110, 462-470.

Vertommen, H., Verheul, R., Ruiter, C. de, \& Hildebrand, M. (2002). Handleiding bij de herziene versie van Hare's Psychopathie Checklist. [Manual of the Dutch version of the revised Hare's Psychopathy Checklist]. Lisse, the Netherlands: Swets Test Publishers.
Viding, E., Blair, R.J.R., Moffitt, T.E., \& Plomin, R. (2005). Evidence for substantial genetic risk for psychopathy in 7-year-olds. Journal of Child Psychology and Psychiatry, 46, 592-597.

Vincent, G.M. (2002). Investigating the legitimacy of adolescent psychopathy assessments: Contributions of item response theory. Unpublished doctoral dissertation. Simon Fraser University, Canada.

Vincent, G.M., Vitacco, M., Grisso, T., \& Corrado, R. (2003). Subtypes of adolescent offenders: Affective traits and antisocial behavior patterns. Behavioral Sciences and the Law, 21, 695-712.

Vitacco, M.J., Neumann, C.S., \& Jackson, R. (2005). Testing a four-factor model and its association with ethnicity, gender, intelligence, and violence. Journal of Consulting and Clinical Psychology, 73, 466-476.

Vitacco, M.J., \& Vincent, G.M. (2006). Understanding the downward extension of psychopathy to youth: Implications for risk assessment and juvenile justice. International Journal of Forensic Mental Health, 5, 29-38. 
Vitale, J.E., \& Newman, J.P. (2001a).

Response perseveration in psychopathic women. Journal of Abnormal

Psychology, 110, 644-647.

Vitale, J.E., \& Newman, J.P. (2001b).

Using the Psychopathy Checklist-Revised with female samples: Reliability, validity, and implications for clinical utility.

Clinical Psychology: Science, \& Practice, 8, 117-132.

Vitale, J.E., Smith, S.S., Brinkley, C.A., \& Newman, J.P. (2002). The reliability and validity of the Psychopathy Checklist-Revised in a sample of female offenders. Criminal Justice and Behavior, 29, 203-231.

Walsh, Z., Allen, L.C., \& Kosson, D.S. (2007). Beyond social deviance: Substance use disorders and the dimensions of psychopathy. Journal of Personality Disorders, 21, 273-288.

Walters, G.D. (2003a). Predicting institutional adjustment and recidivism with the Psychopathy Checklist Factor scores: A meta-analysis. Law and Human Behavior, 27, 541-558.

Walters, G.D. (2003b). Predicting criminal justice outcomes with the Psychopathy
Checklist and Lifestyle Criminality

Screening Form: A meta-analytic comparison. Behavioral Sciences and the Law, 21, 89-102.

Walters, G.D., Knight, R.A., Grann, M., \& Dahle, K.-P. (2008). Incremental validity of the Psychopathy Checklist facet scores: Predicting release outcome in six samples. Journal of Abnormal Psychology, 117, 396-405.

Wartna, B.S., El Harbachi, S., \& Laan, A.M., van der (2005). Jong Vast: Een cijfermatig overzicht van de strafrechtelijke recidive van expupillen van justitiële jeugdinrichtingen. [Incarcerated at a young age: A numeric view of criminal recidivism of exinmates of juvenile justice institutions]. Den Haag: WODC.

Webster, C.D., Douglas, K.S., Eaves, D., \& Hart, S.D. (1997). HCR-20. Assessing the risk of violence. Version 2. Vancouver, BC, Canada, Simon Fraser University and Forensic Psyhiatric Services Commission of British Columbia.

Widiger, T.A., \& Lynam, D.R. (1998). Psychopathy and the five factor model of personality. In T. Million, E. Simonsen, M. Briket-Smith, \& R. D. Davis (Eds.), 
Psychopathy: Antisocial, criminal, and violent behavior. New York: Guilford.

Wijk, A., van, Vermeiren, R., Loeber, R., 't Hart-Kerkhoffs, L., Doreleijers, T., \& Bullens, R. (2006). Juvenile sex offenders compared to non-sex offenders: A review of the literature 1995-2005. Trauma, Violence and Abuse, 7, 227-243.

Wong, S. (1988). Is Hare's Psychopathy Checklist reliable without the interview. Psychological Reports, 62, 931-934.

Wong, S. \& Hare, R.D. (2005). Guidelines for a Psychopathy Treatment Program. Toronto, ON: Multi-Health Systems. Wootton, J.M., Frick, P.J., Shelton, K.K., \& Silverthorn, P. (1997). Ineffective parenting and childhood conduct problems: The moderating role of callousunemotional traits. Journal of Consulting and Clinical Psychology, 65, 301-308.

Zoccolillo, M. (1992). Co-occurence of conduct disorder and its adult outcomes with depressive and anxiety disorders: A review. Journal of the American Academic of Child and Adolescent Psychiatry, 31, 547-556. 
Tot de tanden toe bewapend met een schuimrubberen zwaard van Jort, kraaiende bemoedigingen van Noure en een kus van Jus worstelde ik me door de laatste loodjes die leuker bleken dan verwacht. Het ei is gelegd, het proefschrift is er. Het werd alleen mogelijk door de hulp van belangrijke personen die mij bijstonden in verschillende fasen van mijn promotieonderzoek. Aan jullie dit woord van dank.

Dank aan...

... mijn promotor Corine de Ruiter. Je gaf mij de kans om me in het kader van dit proefschrift in het onderwerp te verdiepen. Jouw passie inspireert, jouw kennis zet aan tot leergierigheid en jouw lef om je nek uit te steken voor de goede zaak is bewonderswaardig. Het was bijzonder om zo veel van je te mogen leren.

... mijn tweede promotor Theo Doreleijers voor je vertrouwen, rust en de inspirerende wijze waarmee je mensen bij elkaar brengt en weet te stimuleren tot bijzondere prestaties.

... de jeugdigen die meewerkten aan het onderzoek. Jullie verdienen een goede toekomst.

... Margot van Heteren die het motiverende vertrouwen in me stelde om een in Jongerenhuis Harreveld onbewandeld pad te bewandelen en me en passant te infecteren met een passie voor de doelgroep.

... Sanne Hillege. Je sleepte mij door de moeilijkste periode van het onderzoek heen door vertrouwen te tonen, taken over te nemen en geïnspireerd te blijven door het onderwerp. Je gepassioneerde ambitie en onze gedeelde zoektocht naar het combineren van wetenschap en klinische praktijk, maken dat onze vriendschap veel langer zal duren dan het onderzoek.

... Henny Lodewijks. In twee verschillende levensfasen waarin ik voor het eerst moeder werd en jij voor het eerst opa, doorliepen we hetzelfde verbindende promotietraject. Dank je voor de gulheid waarmee je deelde in data en wijsheid.

... Het management van Jongerenhuis Harreveld en in het speciaal Marieke Claes die mij door de laatste fase van het promotietraject wilde helpen.

Bedankt voor het ondersteunen van de dataverzameling, zonder welke dit 
proefschrift niet was mogelijk geweest.

... het Ministerie van Justitie voor het beschikbaar stellen van subsidie en het daarmee mogelijk maken van dit onderzoek.

... De Waag voor het gul verstrekken van subsidie aan een nieuwe werknemer, waardoor het mogelijk werd dit proefschrift te laten drukken.

... Wietske Lute dank ik voor het mooie ontwerp van het proefschrift. Zij gebruikte hiervoor het schilderij 'Maskers' van Willy Vittali.

... de leden van de leescommissie Arnoud Arntz, David Bernstein, Andries Korebrits, Chijs van Nieuwenhuizen, Robert Vermeiren en Henk Nijman voor jullie bereidheid tot deelname aan de commissie en het beoordelen van het proefschrift.

... mijn paranimfen Vivienne de Vogel en Anne Maurice van Eck. Vivienne, jij representeert voor mij dé moderne vrouw die het onmogelijke met gemak lijkt te volbrengen: het combineren van een betekenisvolle carrière en het liefdevol grootbrengen van vier fantastische kinderen met alle rust die ze verdienen. Dank voor al je steun. Anne Maurice, ik wil je bedanken voor je woorden en vriendschap in de periode waarin mijn leven op z'n kop gezet werd en daarna. Je stimuleerde me mijn nieuwste carrièredroom te verwezenlijken waardoor we nu collega's zijn en toekomstige avonden aan de eettafel weer voorbij zullen vliegen.

... Wybren Grooteboer, Harry te Walvaart, Roelyn van der Hoek, Jan Willem ter Huurne, Susanne Wormgoor en Stefan Sterrenberg van de voormalige afdeling beleid en strategie van Jongerenhuis Harreveld. Niet alleen lief en leed kon ik met jullie delen, maar ook goede typetjes, anekdotes, fantastische marinade, heerlijke salade Niçoise, juridische raad en zwangerschapskleding. Ingrediënten die van onschatbare waarde zijn gebleken.

... de gedragswetenschappers en test-assistent die ten tijde van het onderzoek werkzaam waren in Jongerenhuis Harreveld: Claudia Francesschina, Mayke Aalbers, Barbara Klein Nagelvoort, Jacqueline van Dijk, Lolita Kapel, Annebeth Mosterd, Calder Rokosch, Christian van Dam, Kees Zwijnenburg, Katelijne Hooijmakers, Erik Masselink, Susana Castilla Carrasco, Trienke Kootstra, Jeanet de Vries, Yvonne Derksen en anderen. Jullie vriendschap en bijdrage aan de dataverzameling (soms zelfs in het weekend) hebben mogelijk 
gemaakt dat ik nu kan promoveren. Het is tijd voor een reünie!

... Manon Stapel, Kathrin Krämer en Voke de Boer voor jullie hulp bij het verzamelen van de data in het kader van jullie werkstuk (al weer heel lang geleden!).

... Lieke van Domburgh, Arne Popma, Sannie Hamerlynck, Marleen Nagtegaal, Yoast van Baardewijk en Sjouk de Boer voor het delen van de leuke en minder leuke beslommeringen tijdens de verschillende fasen van het onderzoek. Yoast, als mede-psychopath buster wil ik wil jou in het speciaal bedanken voor het aanbieden van een werkplek tijdens de maandelijkse woensdagen, voor je bereidheid om mij te helpen aan literatuur en voor het delen van je enthousiasme over het onderwerp.

... Farid Chakksi en Martin Hildebrand voor het beschikbaar stellen van data, waardoor het meest uitdagende gedeelte van het onderzoek mogelijk werd.

... Marjolijn van Casteren, Henny de Wit-Grouls en Myriam Hoorninck voor de gezellige samenwerking tijdens het coderen van de dossiers in de eerste fase van mijn onderzoek.

... Saapke Hoekstra, Bas van der Linden, Harriët Steffens, Willeke van der Bom en andere collega's van de Hogeschool van Amsterdam die het experiment van mij als docent aan de Hogeschool van Amsterdam een unieke ervaring hielpen maken.

... Susan Polak, Jolle Tjaden, Ieke Egas Repáraz, Anne-Marie Terpstra, Stefan Houtman, Marca Geeraets, Marianne Haspels, Gonnie Albrecht, Han Spanjaard en andere collega's van PI-Research voor de korte maar gezellige periode waarin ik de gelegenheid kreeg te ontdekken dat mijn passie elders lag. ... Wim Vulink en Barbara van Roozendaal voor de mogelijkheid me te kunnen ontwikkelen tot een goede psychotherapeut. Mijn nieuwe collega's bij De Waag Almere dank ik voor het hartelijke welkom van de afgelopen maanden. ... Dagmar, voor je levenslange vriendschap. Iedere keer dat we elkaar zien, krijg ik tenminste één keer de slappe lach.

... Ilse. De geboortes van onze kinderen zijn onuitwisbare momenten en ik ben zo blij dat we ze deelden. 
... Serge. Ik kan altijd op je rekenen. Ook in de periode dat zelfs een telefoontje voor mij te veel was.

... Bea voor de uren waarin je, met hulp van Sabrine, Chantal en Mario, zo enthousiast op Jort en Noure past.

... mijn schoonfamilie Frank, Cunera, Lawrence, Kim, Jildar, Clemens en Roos. Bedankt voor jullie steun, het oppassen, de afleiding, de muziek, de biertjes en de gezelligheid tijdens geplande of ongeplande momenten bij het vuur. Echt een tweede thuis!

... mijn ouders Peter en Willie voor alle steun. Jullie liefde heeft me het vertrouwen gegeven dat ik alles kan proberen en dat er altijd een basis is om op terug te vallen. Jullie voorbeeld heeft me geleerd wat er mogelijk is als je doorzet, ook als het niet meezit. Van jullie heb ik geleerd om met een open en respectvolle houding in het leven te staan en oog te hebben voor de bijzondere dingen die op m'n pad komen. Ik geniet jullie te zien genieten van onze kinderen.

... mijn zusje Sharon voor het feit dat je er altijd bent. Er is veel veranderd sinds de tijd dat we een kamer deelden en in bed kletsten over wat ons bezig hield maar onze hechte band is even sterk gebleven. Ik wens jou en Dion alles wat mooi is.

... en alle andere vrienden en familie die mij steeds hebben gesteund. Jullie zijn geweldig!

Als laatste richt ik me tot mijn gezin. Lieve Jort en Noure, ik ben zo trots op jullie. Jullie zijn fantastische, prachtige en slimme kinderen en jullie maken me ontzettend gelukkig. Lieve Justin, dank je voor je daadkrachtige steun. Ik heb vaak willen stoppen met het onderzoek maar jij hield de locomotief in beweging: je hield het eindstation voor ogen, je gooide er brandstof in en je zorgde ervoor dat de route mooi, onvoorspelbaar, liefdevol en bijzonder werd. Precies zoals ik het wil. Ik wil zo graag met je trouwen!! 


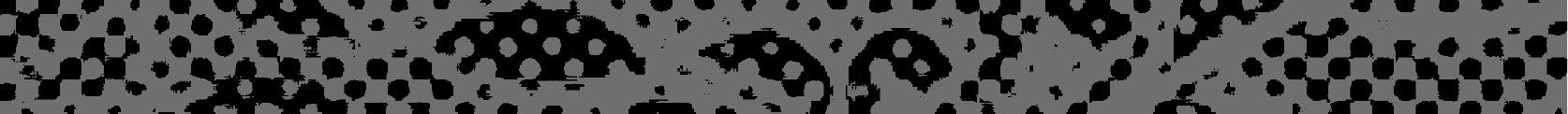

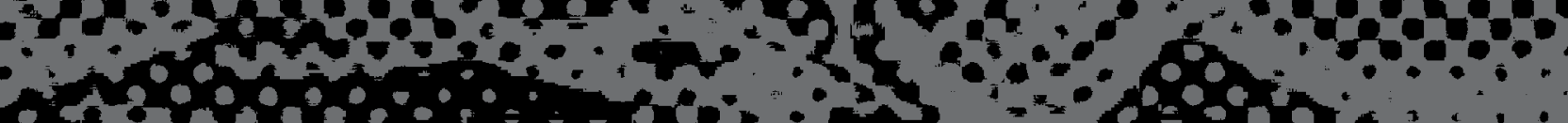

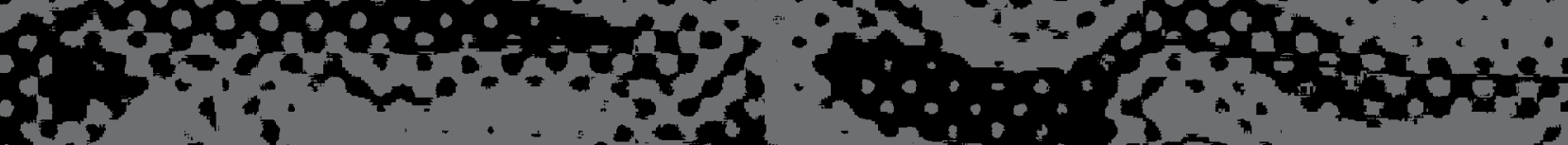

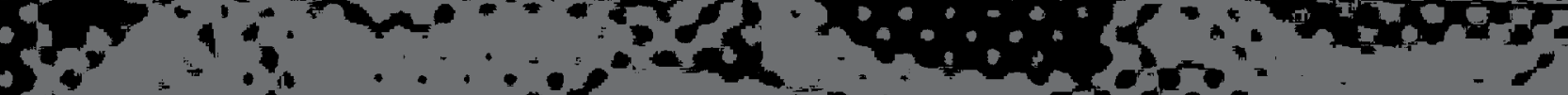
$\because \therefore \because$

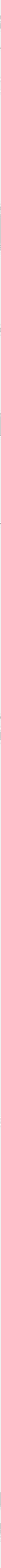


Jacqueline (Jacky) Das was born September $16^{\text {th }}, 1979$ in Naarden, The Netherlands. She attended high school (Atheneum) in Hilversum from 1991 until 1997. In September 1997, she began her Psychology studies at the University of Amsterdam and obtained her Master's degree (Met genoegen) in 2001. During her studies, she specialized in the area of forensic psychology by conducting her clinical internship at Jongerenhuis Harreveld, a juvenile justice treatment institution. Furthermore, she performed research on offender profiling for her Master's thesis at the Dr. Henri van der Hoeven Kliniek, a forensic psychiatric hospital in Utrecht, The Netherlands. As of October 2001, she started her dissertation research on psychopathic traits in adolescents at Jongerenhuis Harreveld. Currently, she provides training on the assessment of psychopathic traits in youth and works as a psychologist at outpatient forensic psychiatric center De Waag, Almere, The Netherlands. 


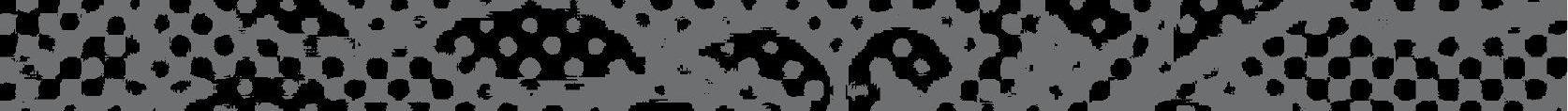

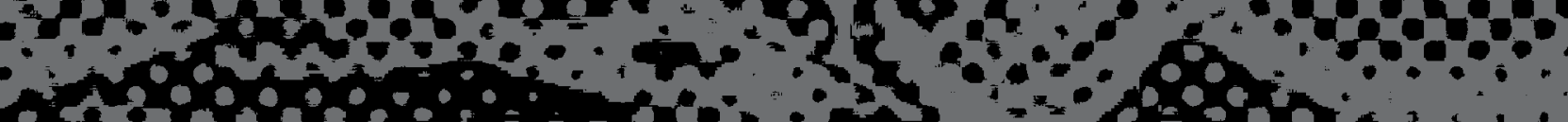

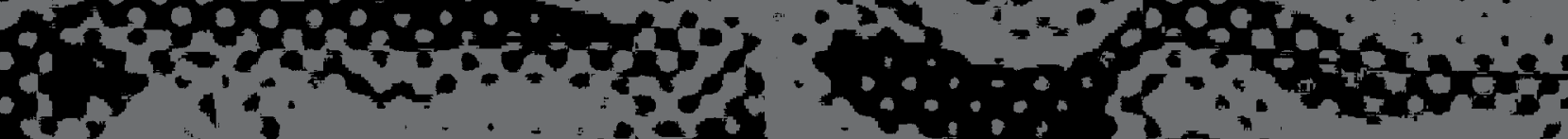

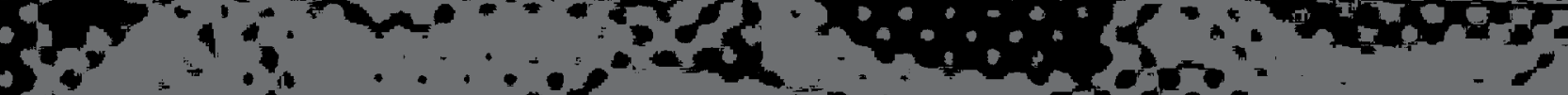
$\because \therefore-a$

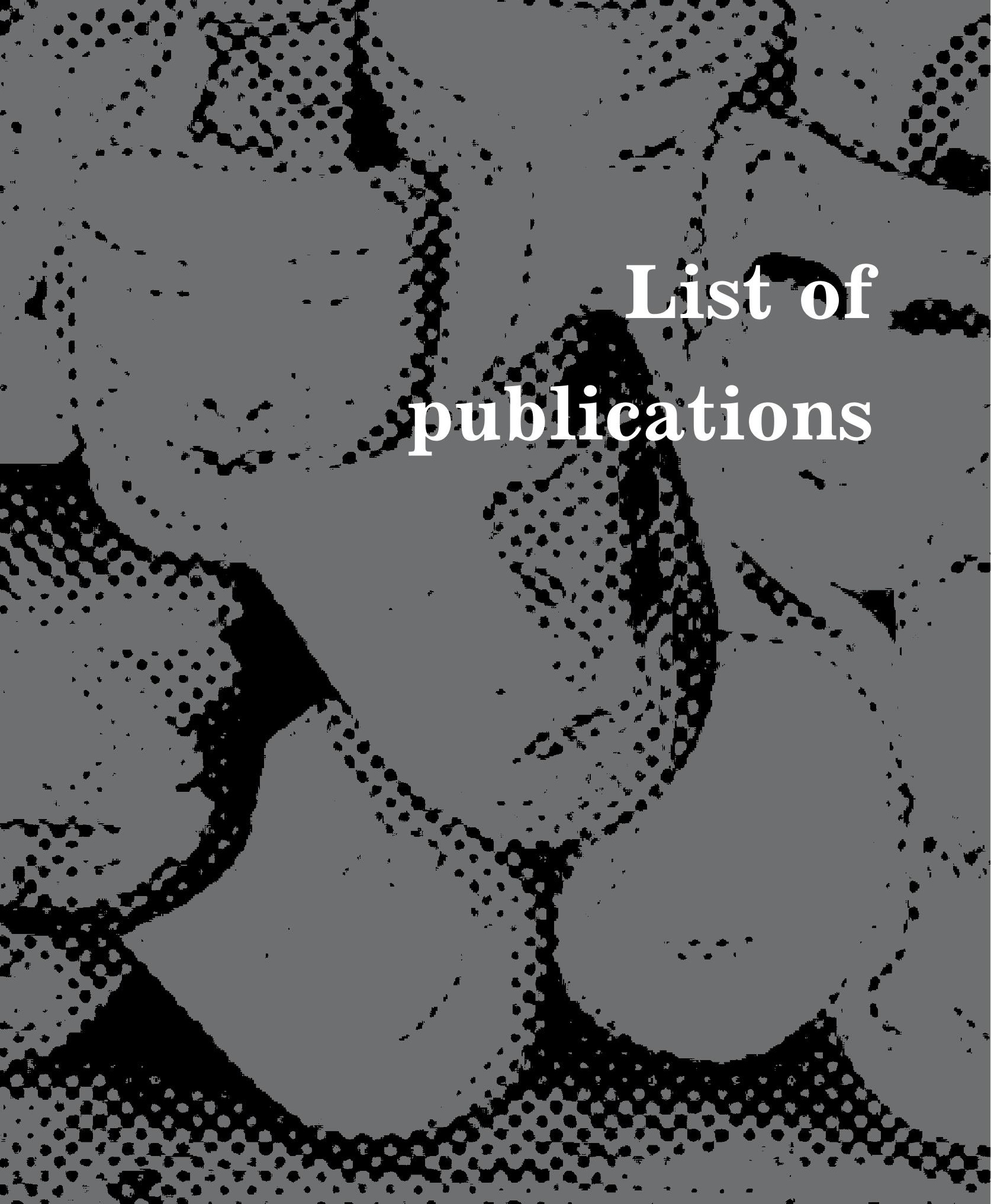




\section{In Dutch}

Das, J. (2007). A safe society. IAFMHS-conferentie, Amsterdam, 13-16 juni 2006. [A safe society. IAFMHS-conference, Amsterdam, 13-16 June 2006]. Tijdschrift voor Psychotherapie, 33, 159-161.

Das, J., \& de Ruiter, C. (2003). Wet en waan [Law and delusion: Report on the ILAMH congress]. Verslag van het XVIIth International Congress on Law and Mental Health, 8-12 juli 2002, Amsterdam. Maandblad Geestelijke volksgezondheid, 58, 174-176.

Das, J., Ruiter, C. de, Heteren, M. van, \& Doreleijers, Th. (2004). Psychopathie bij kinderen en jeugdigen: Stand van zaken en diagnostische instrumenten. Tijdschrift voor Orthopedagogiek, Kinderpsychiatrie en Klinische Kinderpsychologie, 29, 30-43.

\section{In English}

Das, J., Ruiter, C. de, \& Doreleijers, Th. (2008). Reliability and validity of the Psychopathy Checklist: Youth Version in Dutch female adolescents. International Journal of Law and Psychiatry, 31, 219-228.

Das, J., Ruiter, C. de, Lodewijks, H., \& Doreleijers, T. (2007). Predictive validity of the Dutch PCL:YV for institutional disruptive behavior: Findings from two samples of male adolescents in a juvenile justice treatment institution. Behavioral Sciences and the Law, 2, 739-755. 


\section{Translations}

Bullens, R.A.R., Horn, J.E., Eck, A.J., van, \& Das, J. (2005). J-SOAP $D$ : De Nederlandse vertaling en bewerking van de J-SOAP II (Juvenile Sex Offender Assessment Protocol II). Handleiding, Versie 1. [J-SOAP D: The Ducth translation and revision of the J-SOAP II (Juvenile Sex Offender Assessment Protocol II). Manual, Version I]. Utrecht: Forum Educatief.

Das, J., \& Ruiter, C. de (2003). Youth Psychopathic traits Inventory: geautoriseerde Nederlandse vertaling. [Youth Psychopathic traits Inventory: Authorized Dutch translation]. Unpublished research version.

Ruiter, C. de, Das, \& Domburgh, L., van (2003). Antisocial Process Screening Device: Geautoriseerde Nederlandse vertaling [Antisocial Process Screening Device: Authorized Dutch Translation]. Unpublished Research Version.

Ruiter, C. de, Kuin, N., Vries, J. de, \& Das, J (2002). Psychopathie Checklist: Jeugd Versie: Geautoriseerde Nederlandse vertaling. [Psychopathy Checklist: Youth Version: Authorized Dutch translation]. Unpublished research version. 
